\title{
Asymmetric Full Saturation of Vinylarenes with Cooperative Homogeneous and Heterogeneous Rhodium Catalysis
}

Haibo Wu, ${ }^{1 \S}$ Jianping Yang, ${ }^{1 \S}$ Bram B. C. Peters, ${ }^{1}$ Luca Massaro, ${ }^{1}$ Jia Zheng ${ }^{1}$ and Pher G. Andersson ${ }^{* 1,2}$

${ }^{1}$ Department of Organic Chemistry, Stockholm University, Svante Arrhenius väg 16C, SE-10691 Stockholm, Sweden.

${ }^{2}$ School of Chemistry and Physics, University of Kwazulu-Natal, Private Bag X54001, Durban, 4000, South Africa.

§These authors contributed equally to this work.

*Corresponding author. Email: pher.andersson@su.se.

\section{Contents}

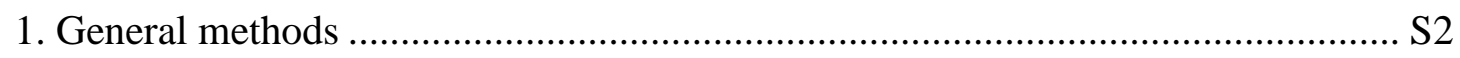

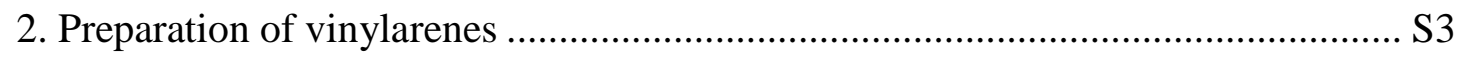

3. Development of arene hydrogenation based on $\mathrm{Rh} /$ diphosphine system and kinetic studies S10

4. Evaluation of Rh-precursors and the generality of diphosphine ligand. S11

5. General procedure for asymmetric hydrogenation of arenes S12

6. Assignment of the absolute configurations of hydrogenated products. S29

7. Scale-up asymmetric hydrogenations and applications S30

8. Additional experiments and HR-TEM images of in-situ generated Rhnanoparticles.

9. ${ }^{1} \mathrm{H},{ }^{13} \mathrm{C}$ and ${ }^{1} \mathrm{~F}$ NMR spectra for new compounds S35

10. GC Chromatograms for hydrogenated products S110

11. References S121 


\section{General methods}

Unless otherwise noted, all reactions were conducted under dry nitrogen or argon atmosphere using magnetic stirring. $i \mathrm{PrOH}$ was freshly distilled from magnesium under nitrogen $\mathrm{CH}_{2} \mathrm{Cl}_{2}$ was freshly distilled from magnesium under nitrogen; THF was freshly distilled from $\mathrm{Na}$ and benzophenone under argon; All other solvents and reagents were purchased from commercial suppliers and used without further purification. Chromatographic separations were performed on Kiesel gel $60 \mathrm{H}$ silica gel (particle) size: 0.063-0.100 mm). Thin layer chromatography (TLC) was performed on aluminium plates coated with Kieselgel $60(0.20 \mathrm{~mm}$, UV254) and visualized under ultraviolet light $(v=254 \mathrm{~nm})$, or by staining with ethanolic phosphomolybdic acid and heating. ${ }^{1} \mathrm{H}$ NMR spectra were recorded at $400 \mathrm{MHz}$ in Chloroform- $d$ at $25^{\circ} \mathrm{C}$ and referenced internally to the residual $\mathrm{CHCl}_{3}$ peak (7.26 ppm) or were recorded at $400 \mathrm{MHz}$ in DMSO- $d_{6}$ at $25^{\circ} \mathrm{C}$ and referenced internally to the residual DMSO peaks (2.50 ppm). ${ }^{13} \mathrm{C}$ NMR spectra were recorded at $100 \mathrm{MHz}$ in Chloroform- $d$ at $25{ }^{\circ} \mathrm{C}$ and referenced to the central peak of Chloroform- $d$ (77.16 ppm) or were recorded at $100 \mathrm{MHz}$ in DMSO- $d_{6}$ at $25{ }^{\circ} \mathrm{C}$ and referenced to the central peak of DMSO (39.52 ppm). ${ }^{19} \mathrm{~F}$ NMR spectra were recorded at $377 \mathrm{MHz}$ in Chloroform- $d$ or DMSO- $d_{6}$ at $25^{\circ} \mathrm{C}$. Chemical shifts are reported in ppm ( $\delta$ scale). Optical rotations were recorded on a thermostated polarimeter using a sodium lamp $(589 \mathrm{~nm})$ and a $1.0 \mathrm{dm}$ cell. Enantiomeric excesses and diastereomeric ratios were determined using GC (30 m columns, Helium gas carrier at $1 \mathrm{~mL} / \mathrm{min}$, constant pressure) with a MS detector. Racemic compounds were used for comparison. HRMS was performed on a Bruker microTOF with an ESI source.

Ligands L1-L7 were used as purchased from Strem Chemicals Inc. and Merck-Sigma Aldrich. Ligand L8 $[(R, S, R, S)$-Me-PennPhos] and L9 were synthesized according to the literature. ${ }^{1}$

$\left[\mathrm{Rh}(\mathrm{COD})_{2}\right] \mathrm{PF}_{6}$ was synthesized according to the literature. ${ }^{2}$

$\left[\mathrm{Rh}(\mathrm{COD})_{2}\right] \mathrm{BAr}_{\mathrm{F}}$ was synthesized according to the literature. ${ }^{3}$

The other rhodium precursors were used as purchased from Merck-Sigma Aldrich and Strem Chemicals Inc. 


\section{Preparation of vinylarenes}

General procedure for the synthesis of synthesis of enamides

Method A (for acetal-amides):

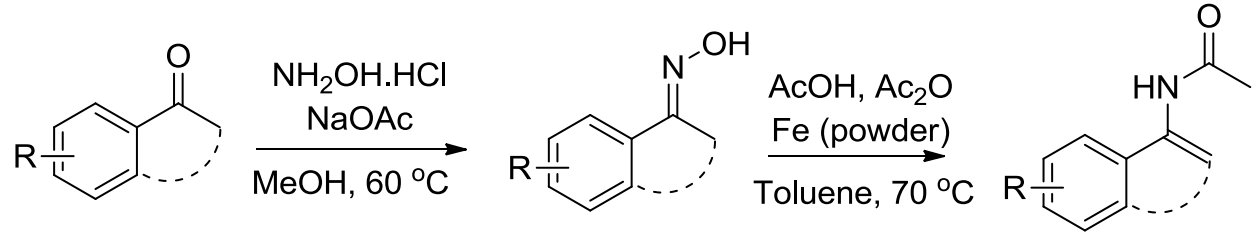

Method B (for the other enamides):

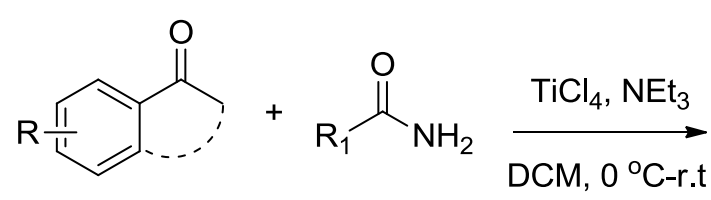<smiles>[R]CNC(=O)C1=CC=[R]=CC=C1</smiles>

Acetal-enamides $\mathbf{4 c}, \mathbf{4 d}, \mathbf{4 k}, \mathbf{1 b}, \mathbf{1 j}$ were prepared following method A, which was carried out according to the literature. ${ }^{4}$ The other enamides $4 \mathbf{b}, \mathbf{4 f}, \mathbf{4 g}, \mathbf{4 i}, \mathbf{4 j}, \mathbf{1 a}, \mathbf{1 c}$, 1d, 1e, 1f, 1g, 11 were prepared following method B, which was carried out according to the literature. ${ }^{5}$

Characterization for the following compounds have been previously reported: $\mathbf{4 f}{ }^{6}$ $4 \mathbf{g},{ }^{6} 4 \mathbf{k},{ }^{7} 1 \mathbf{k},{ }^{8} 1 \mathbf{l}^{8}$

New compounds:

\section{2,2,2-trifluoro- $N$-(1-(p-tolyl)vinyl)acetamide}<smiles>C=C(NC(=O)C(F)(F)F)c1ccc(C)cc1</smiles>

White Solid. ${ }^{1} \mathrm{H}$ NMR (400 MHz, DMSO- $d_{6}$ ) $\delta 10.9$ (s, $b r$, $1 \mathrm{H}), 7.3(\mathrm{~d}, J=8.07 \mathrm{~Hz}, 2 \mathrm{H}), 7.2(\mathrm{~d}, J=8.03 \mathrm{~Hz}, 2 \mathrm{H}), 5.5(\mathrm{~s}$, $1 \mathrm{H}), 5.4(\mathrm{~s}, 1 \mathrm{H}), 2.3(\mathrm{~s}, 3 \mathrm{H}) .{ }^{13} \mathrm{C}$ NMR (100 MHz, DMSO- $\left.d_{6}\right)$ $\delta 155.4(\mathrm{q}, J=36.81 \mathrm{~Hz}), 139.1,138.3,133.0,129.1,125.6,115.9(\mathrm{q}, J=288.61 \mathrm{~Hz})$, 108.8, 20.7. ${ }^{19} \mathrm{~F}$ NMR (377 MHz, DMSO- $\left.d_{6}\right) \delta-73.95$. HRMS-ESI: Found $[\mathrm{M}+\mathrm{Na}]^{+}=$ 252.0628; $\mathrm{C}_{11} \mathrm{H}_{10} \mathrm{~F}_{3} \mathrm{NONa}$ requires 252.0607. 


\section{2,2,2-trifluoro- $N$-(1-(m-tolyl)vinyl)acetamide}

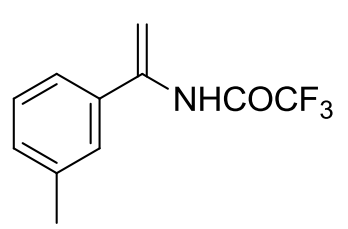

Colorless oil. ${ }^{1} \mathrm{H}$ NMR (400 MHz, DMSO- $\left.d_{6}\right) \delta 10.87$ (s, $b r$, $1 \mathrm{H}), 7.33-7.25(\mathrm{~m}, 2 \mathrm{H}), 7.25-7.17(\mathrm{~m}, 2 \mathrm{H}), 5.56(\mathrm{~s}, 1 \mathrm{H})$, $5.46(\mathrm{~s}, 1 \mathrm{H}), 2.33(\mathrm{~s}, 3 \mathrm{H}),{ }^{13} \mathrm{C}$ NMR (100 MHz, DMSO-d $) \delta$ $155.4(\mathrm{q}, J=36.73 \mathrm{~Hz}), 139.2,137.7,135.9,129.4,128.4$, $126.3,122.9,115.6$ (q, $J=288.98 \mathrm{~Hz}), 109.3,21.0 .{ }^{19} \mathrm{~F}$ NMR (377 MHz, DMSO- $\left.d_{6}\right)$ $\delta$-73.94. HRMS-ESI: Found $[\mathrm{M}+\mathrm{Na}]^{+}=252.0609 ; \mathrm{C}_{11} \mathrm{H}_{10} \mathrm{~F}_{3} \mathrm{NONa}$ requires 252.0607.

\section{$N-(1-(o-$ tolyl)vinyl)pivalamide}

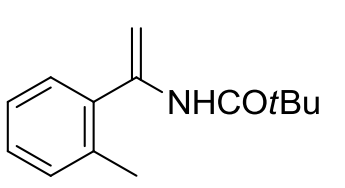

White solid. ${ }^{1} \mathrm{H}$ NMR (400 MHz, DMSO- $\left.d_{6}\right) \delta 8.79$ (s, $\left.b r, 1 \mathrm{H}\right)$, $7.27-7.14(\mathrm{~m}, 4 \mathrm{H}), 5.68(\mathrm{~s}, 1 \mathrm{H}), 4.53(\mathrm{~s}, 1 \mathrm{H}), 2.25(\mathrm{~s}, 3 \mathrm{H})$, $1.14(\mathrm{~s}, 9 \mathrm{H}) .{ }^{13} \mathrm{C}$ NMR (100 MHz, DMSO-d $) \delta 176.4,142.4$, 139.2, 135.3, 129.9, 128.9, 127.7, 125.5, 103.3, 39.1, 27.1, 19.5. HRMS-ESI: Found $[\mathrm{M}+\mathrm{Na}]^{+}=240.1379 ; \mathrm{C}_{14} \mathrm{H}_{19} \mathrm{NONa}$ requires 240.1359 .

\section{4-methyl- $N$-(1-phenylvinyl)benzamide}<smiles>C=C(NC(=O)c1ccc(C)cc1)c1ccccc1</smiles>

White solid. ${ }^{1} \mathrm{H}$ NMR (400 MHz, DMSO- $\left.d_{6}\right) \delta 9.82(\mathrm{~s}, b r$, $1 \mathrm{H}), 7.88(\mathrm{~d}, J=5.51 \mathrm{~Hz}, 2 \mathrm{H}), 7.49(\mathrm{~d}, J=4.09 \mathrm{~Hz}, 2 \mathrm{H})$, $2.13 \mathrm{~Hz}, 1 \mathrm{H}), 2.37$ (s, 3H). ${ }^{13} \mathrm{C}$ NMR (100 MHz, DMSO-d $) \delta 165.8,141.9,141.5$, 138.0, 131.7, 128.9, 128.3, 128.1, 127.8, 126.0, 105.7, 21.0. HRMS-ESI: Found $[\mathrm{M}+\mathrm{Na}]^{+}=260.1057 ; \mathrm{C}_{16} \mathrm{H}_{15} \mathrm{NONa}$ requires 260.1046.

\section{$N$-(1-(p-tolyl)vinyl)benzamide}<smiles>C=C(NC(=O)c1ccccc1)c1ccc(C)cc1</smiles>

White solid. ${ }^{1} \mathrm{H}$ NMR (400 MHz, DMSO- $\left.d_{6}\right) \delta 9.82$ (s, $b r$, $1 \mathrm{H}), 7.94(\mathrm{dd}, J=7.09,1.46 \mathrm{~Hz}, 2 \mathrm{H}), 7.61-7.53(\mathrm{~m}, 1 \mathrm{H})$, $7.53-7.45(\mathrm{~m}, 2 \mathrm{H}), 7.43-7.34(\mathrm{~m}, 2 \mathrm{H}), 7.18(\mathrm{~d}, J=7.91$ $\mathrm{Hz}, 2 \mathrm{H}), 5.46$ (s, 1H), 5.27 (s, 1H), 2.31 (s, 3H). ${ }^{13} \mathrm{C}$ NMR (100 MHz, DMSO-d6) $\delta$ 165.9, 141.7, 137.5, 135.1, 134.5, 131.5, 128.8, 128.3, 127.7, 125.8, 105.1, 20.7. HRMS-ESI: Found $[\mathrm{M}+\mathrm{Na}]^{+}=260.1063 ; \mathrm{C}_{16} \mathrm{H}_{15} \mathrm{NONa}$ requires 260.1046. 


\section{$N$-(1H-inden-3-yl)isobutyramide}

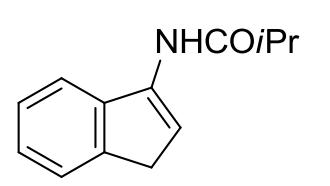

White solid. ${ }^{1} \mathrm{H}$ NMR (400 MHz, Chloroform- $d$ ) $\delta 9.61$ (s, br, 1H), $7.78(\mathrm{~d}, J=7.60 \mathrm{~Hz}, 1 \mathrm{H}), 7.46(\mathrm{~d}, J=7.32 \mathrm{~Hz}, 1 \mathrm{H}), 7.32$ (td, $J=7.55,0.98 \mathrm{~Hz}, 1 \mathrm{H}), 7.22(\mathrm{td}, J=7.39,0.94 \mathrm{~Hz}, 1 \mathrm{H}), 6.76(\mathrm{t}, J$ $=2.31 \mathrm{~Hz}, 1 \mathrm{H}), 3.37(\mathrm{~d}, J=2.33 \mathrm{~Hz}, 2 \mathrm{H}), 2.81($ hept $, J=6.79 \mathrm{~Hz}, 1 \mathrm{H}), 1.11(\mathrm{~d}, J=$ $6.81 \mathrm{~Hz}, 6 \mathrm{H}) .{ }^{13} \mathrm{C}$ NMR (100 MHz, Chloroform- $\left.d\right) \delta 175.9,142.3,140.1,136.7,125.7$, 125.0, 123.8, 118.3, 114.1, 35.9, 34.0, 19.6. HRMS-ESI: Found $[\mathrm{M}+\mathrm{Na}]^{+}=224.1046$; $\mathrm{C}_{13} \mathrm{H}_{15} \mathrm{NONa}$ requires 224.1067.

\section{$N$-(1H-inden-3-yl)pivalamide}

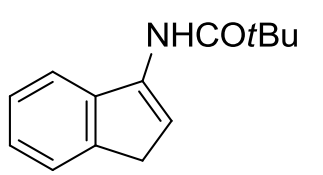

White solid. ${ }^{1} \mathrm{H}$ NMR (400 MHz, DMSO- $\left.d_{6}\right) \delta 8.90(\mathrm{~s}, b r, 1 \mathrm{H})$, $7.67(\mathrm{~d}, J=7.49 \mathrm{~Hz}, 1 \mathrm{H}), 7.46(\mathrm{~d}, J=7.25 \mathrm{~Hz}, 1 \mathrm{H}), 7.31(\mathrm{t}, J=$ $7.38 \mathrm{~Hz}, 1 \mathrm{H}), 7.26-7.15(\mathrm{~m}, 1 \mathrm{H}), 6.65$ (t, $J=2.37 \mathrm{~Hz}, 1 \mathrm{H}), 3.37$ $(\mathrm{d}, J=1.88 \mathrm{~Hz}, 2 \mathrm{H}), 1.27$ (s, 9H). ${ }^{13} \mathrm{C}$ NMR (100 MHz, DMSO- $\left.d_{6}\right) \delta 176.8,142.4$, 140.4, 136.9, 125.6, 125.0, 123.8, 118.8, 116.6, 39.1, 35.8, 27.3. HRMS-ESI: Found $[\mathrm{M}+\mathrm{Na}]^{+}=238.1230 ; \mathrm{C}_{14} \mathrm{H}_{17} \mathrm{NONa}$ requires 238.1202.

\section{2,2,2-trifluoro- $N$-(1H-inden-3-yl)acetamide}

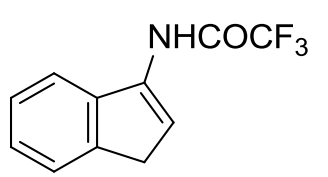

White solid. ${ }^{1} \mathrm{H}$ NMR (400 MHz, Chloroform- $d$ ) $\delta 8.05$ (s, br, $1 \mathrm{H}), 7.52(\mathrm{dd}, J=7.39,2.52 \mathrm{~Hz}, 1 \mathrm{H}), 7.40-7.31(\mathrm{~m}, 2 \mathrm{H}), 7.30-$ $7.25(\mathrm{~m}, 1 \mathrm{H}), 6.98(\mathrm{t}, J=2.47 \mathrm{~Hz}, 1 \mathrm{H}), 3.48(\mathrm{~d}, J=2.52 \mathrm{~Hz}, 2 \mathrm{H})$. ${ }^{13} \mathrm{C}$ NMR (100 MHz, Chloroform- $d$ ) $\delta 155.2$ (q, $\left.J=38.72 \mathrm{~Hz}\right), 142.6,138.5,133.4$, 126.5, 126.3, 124.7, 119.8, 116.2, 115.8 (q, $J=261.51 \mathrm{~Hz}), 36.9 .{ }^{19} \mathrm{~F}$ NMR $(377 \mathrm{MHz}$, Chloroform- $d$ ) $\delta$-75.54. HRMS-ESI: Found $[\mathrm{M}+\mathrm{Na}]^{+}=250.0468 ; \mathrm{C}_{11} \mathrm{H}_{8} \mathrm{~F}_{3} \mathrm{NONa}$ requires 250.0450 .

\section{$N$-(2-methyl-1H-inden-3-yl)pivalamide}

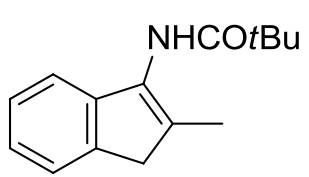

White solid. ${ }^{1} \mathrm{H}$ NMR (400 MHz, Chloroform- $d$ ) $\delta 7.34(\mathrm{~d}, J=$ $7.32 \mathrm{~Hz}, 1 \mathrm{H}), 7.23(\mathrm{t}, J=7.58 \mathrm{~Hz}, 1 \mathrm{H}), 7.13(\mathrm{td}, J=7.43,1.17$ $\mathrm{Hz}, 1 \mathrm{H}), 7.08(\mathrm{~d}, J=7.51 \mathrm{~Hz}, 1 \mathrm{H}), 3.34(\mathrm{~s}, 2 \mathrm{H}), 2.03$ (s, 3H), 1.38 (s, 9H). ${ }^{13} \mathrm{C}$ NMR (100 MHz, Chloroform- $d$ ) $\delta$ 177.1, 142.8, 141.1, 136.1, 131.6, 126.3, 124.5, 123.6, 117.7, 40.9, 39.5, 28.0, 14.2. HRMS-ESI: Found $[\mathrm{M}+\mathrm{Na}]^{+}=$ 252.1386; $\mathrm{C}_{15} \mathrm{H}_{19} \mathrm{NONa}$ requires 252.1376 . 


\section{$N$-(2-ethyl-1H-inden-3-yl)pivalamide}

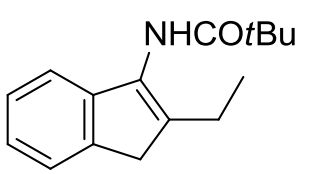

White solid. ${ }^{1} \mathrm{H}$ NMR (400 MHz, Chloroform- $d$ ) $\delta 7.36(\mathrm{~d}, J=$ $7.36 \mathrm{~Hz}, 1 \mathrm{H}), 7.23(\mathrm{~d}, J=7.53 \mathrm{~Hz}, 1 \mathrm{H}), 7.14(\mathrm{td}, J=7.41,1.20$ $\mathrm{Hz}, 1 \mathrm{H}), 7.10(\mathrm{dd}, J=7.46,0.98 \mathrm{~Hz}, 1 \mathrm{H}), 3.35(\mathrm{~d}, J=2.55 \mathrm{~Hz}$, 2H), $2.44(\mathrm{q}, J=15.26 \mathrm{~Hz}, 2 \mathrm{H}), 1.37(\mathrm{~s}, 9 \mathrm{H}), 1.17(\mathrm{t}, J=7.61 \mathrm{~Hz}, 3 \mathrm{H}) .{ }^{13} \mathrm{C} \mathrm{NMR}$ (100 MHz, Chloroform- $d$ ) $\delta 177.1,142.8,141.9,141.2,130.8,126.3,124.5,123.7$, 117.9, 39.5, 38.2, 28.0, 21.6, 13.4. HRMS-ESI: Found $[\mathrm{M}+\mathrm{Na}]^{+}=266.1528$; $\mathrm{C}_{16} \mathrm{H}_{21} \mathrm{NONa}$ requires 266.1515.

\section{2,2,2-trifluoro- $N$-(6-methyl-1H-inden-3-yl)acetamide}

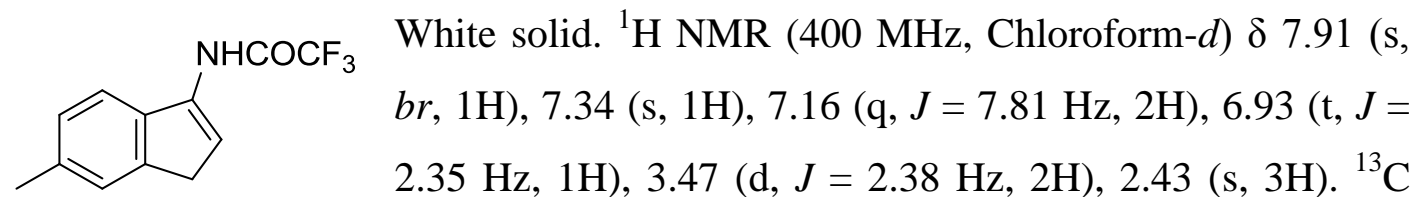

NMR (100 MHz, Chloroform- $d$ ) $\delta 148.2$ (q, $J=51.18 \mathrm{~Hz}), 142.9,136.3,135.9,133.3$, 127.2, 125.7, 118.6, 115.7, 114.5 (q, $J=286.89 \mathrm{~Hz}), 36.7,21.6 .{ }^{19} \mathrm{~F}$ NMR $(377 \mathrm{MHz}$, Chloroform- $d$ ) $\delta$-75.54. HRMS-ESI: Found $[\mathrm{M}+\mathrm{Na}]^{+}=264.0626 ; \mathrm{C}_{12} \mathrm{H}_{10} \mathrm{~F}_{3} \mathrm{NONa}$ requires 264.0607 .

\section{2,2,2-trifluoro- $N$-(7-methyl-1H-inden-3-yl)acetamide}

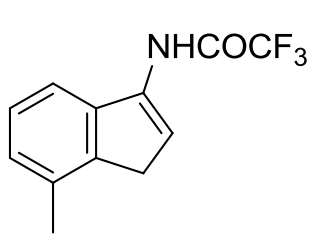

White solid. ${ }^{1} \mathrm{H}$ NMR (400 MHz, Chloroform- $d$ ) $\delta 7.94$ (s, br, $1 \mathrm{H}), 7.29(\mathrm{dd}, J=15.35,7.76 \mathrm{~Hz}, 1 \mathrm{H}), 7.12(\mathrm{dd}, J=9.99,7.53$ $\mathrm{Hz}, 2 \mathrm{H}), 7.01(\mathrm{t}, J=2.49 \mathrm{~Hz}, 1 \mathrm{H}), 3.40(\mathrm{~s}, 2 \mathrm{H}), 2.40(\mathrm{~s}, 3 \mathrm{H}) .{ }^{13} \mathrm{C}$ NMR (100 MHz, Chloroform- $d$ ) $\delta 155.1$ (q, $J=34.80 \mathrm{~Hz}), 141.2$, 138.1, 134.1, 133.6, 127.6, 126.9, 119.2, 115.9 (q, $J=288.64 \mathrm{~Hz}), 113.7,35.9$, 18.5. ${ }^{19} \mathrm{~F}$ NMR (377 MHz, Chloroform- $d$ ) $\delta$-75.51. HRMS-ESI: Found $[\mathrm{M}+\mathrm{Na}]^{+}=$ 264.0622; $\mathrm{C}_{12} \mathrm{H}_{10} \mathrm{~F}_{3} \mathrm{NONa}$ requires 264.0607.

\section{2,2,2-trifluoro- $N$-(5-methoxy- $1 H$-inden-3-yl)acetamide}

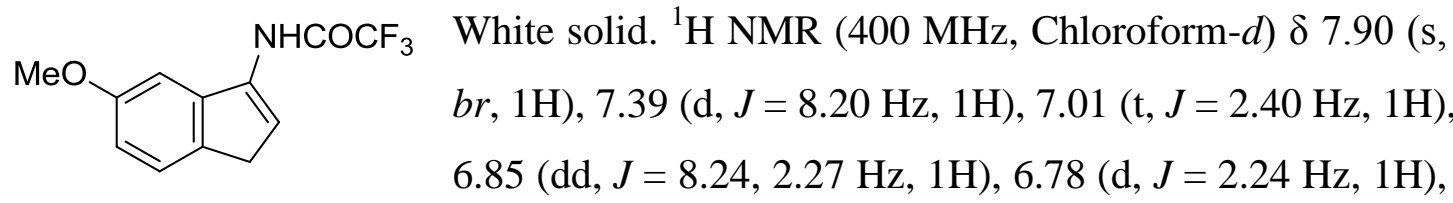
$3.86(\mathrm{~s}, 3 \mathrm{H}), 3.43(\mathrm{~d}, J=2.32 \mathrm{~Hz}, 2 \mathrm{H}) .{ }^{13} \mathrm{C}$ NMR $(100 \mathrm{MHz}$, Chloroform- $d$ ) $\delta 159.1$, $155.1(\mathrm{q}, J=38.27 \mathrm{~Hz}), 139.8,134.6,133.2,125.2,121.3,115.9$ (q, $J=289.60 \mathrm{~Hz})$, 
111.7, 102.6, 55.8, 36.2. ${ }^{19} \mathrm{~F}$ NMR (377 MHz, Chloroform- $d$ ) $\delta$-75.5. HRMS-ESI: Found $[\mathrm{M}+\mathrm{Na}]^{+}=280.0569 ; \mathrm{C}_{12} \mathrm{H}_{10} \mathrm{~F}_{3} \mathrm{NO}_{2} \mathrm{Na}$ requires 280.0556 .

\section{$N$-(7-ethyl-1H-inden-3-yl)-2,2,2-trifluoroacetamide}

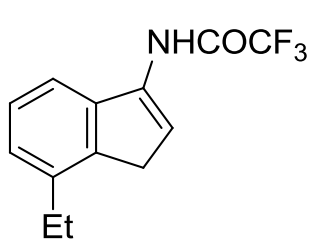

White solid. ${ }^{1} \mathrm{H}$ NMR (400 MHz, Chloroform- $d$ ) $\delta 7.97$ (s, br, $1 \mathrm{H}), 7.34(\mathrm{t}, J=7.58 \mathrm{~Hz}, 1 \mathrm{H}), 7.18(\mathrm{~d}, J=7.56 \mathrm{~Hz}, 1 \mathrm{H}), 7.12$ (dd, $J=7.58,0.94 \mathrm{~Hz}, 1 \mathrm{H}), 7.00(\mathrm{t}, J=2.41 \mathrm{~Hz}, 1 \mathrm{H}), 3.44(\mathrm{~d}, J=$ $2.43 \mathrm{~Hz}, 2 \mathrm{H}), 2.76(\mathrm{q}, J=7.61 \mathrm{~Hz}, 2 \mathrm{H}), 1.30(\mathrm{t}, J=7.61 \mathrm{~Hz}, 3 \mathrm{H})$.

${ }^{13} \mathrm{C}$ NMR (100 MHz, Chloroform- $d$ ) $\delta 155.1(\mathrm{~d}, J=37.73 \mathrm{~Hz}), 140.5,140.2,138.3$, 133.6, 127.1, 125.8, 119.1, 115.7 (q, $J=288.82 \mathrm{~Hz}$ ), 113.7, 35.5, 25.8, 14.3. ${ }^{19} \mathrm{~F}$ NMR (377 MHz, Chloroform- $d$ ) $\delta-75.53$. HRMS-ESI: Found $[\mathrm{M}+\mathrm{Na}]^{+}=278.0784$; $\mathrm{C}_{13} \mathrm{H}_{12} \mathrm{~F}_{3} \mathrm{NONa}$ requires 278.0763 .

\section{$N$-(3,4-dihydronaphthalen-1-yl)pivalamide}

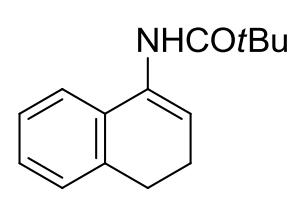

White solid. ${ }^{1} \mathrm{H}$ NMR (400 MHz, DMSO- $\left.d_{6}\right) \delta 8.72$ (s, br, $\left.1 \mathrm{H}\right)$, $7.16(\mathrm{~d}, J=6.35 \mathrm{~Hz}, 3 \mathrm{H}), 7.03(\mathrm{~d}, J=6.21 \mathrm{~Hz}, 1 \mathrm{H}), 5.94(\mathrm{t}, J=$ $4.28 \mathrm{~Hz}, 1 \mathrm{H}), 2.71(\mathrm{t}, J=7.93 \mathrm{~Hz}, 2 \mathrm{H}), 2.33-2.23(\mathrm{~m}, 2 \mathrm{H}), 1.22$ (s, 9H). ${ }^{13} \mathrm{C}$ NMR (100 MHz, DMSO- $\left.d_{6}\right) \delta 176.9,135.8,132.9,132.6,127.3,127.0$, 126.1, 122.1, 121.9, 38.7, 27.4, 27.2, 21.9. HRMS-ESI: Found $[\mathrm{M}+\mathrm{Na}]^{+}=252.1367$; $\mathrm{C}_{15} \mathrm{H}_{19} \mathrm{NONa}$ requires 252.1359 .

\section{$N$-(2H-chromen-4-yl)-2,2,2-trifluoroacetamide}

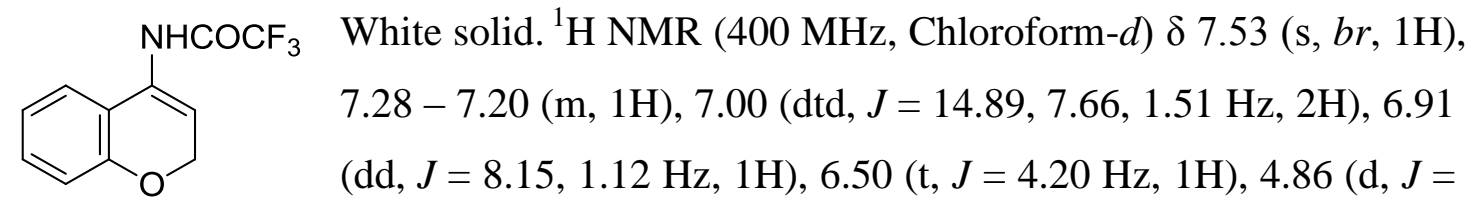
$4.26 \mathrm{~Hz}, 2 \mathrm{H}) .{ }^{13} \mathrm{C}$ NMR (100 MHz, Chloroform- $d$ ) $\delta 155.7$ (q, $J=37.41 \mathrm{~Hz}$ ), 155.3, $130.8,127.0,121.8,119.8,118.9,117.3,115.7$ (q, $J=289.02 \mathrm{~Hz}), 112.2,64.7 .{ }^{19} \mathrm{~F}$ NMR (377 MHz, Chloroform- $d$ ) $\delta$-75.73. HRMS-ESI: Found $[\mathrm{M}+\mathrm{Na}]^{+}=266.0405$; $\mathrm{C}_{11} \mathrm{H}_{8} \mathrm{~F}_{3} \mathrm{NO}_{2} \mathrm{Na}$ requires 266.0399.

\section{$N$-(2H-chromen-4-yl)pivalamide}

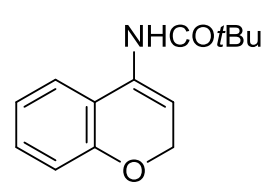

White solid. ${ }^{1} \mathrm{H}$ NMR (400 MHz, Chloroform- $d$ ) $\delta 8.71(\mathrm{~s}, b r, 1 \mathrm{H})$, $7.16(\mathrm{td}, J=7.83,1.62 \mathrm{~Hz}, 1 \mathrm{H}), 7.06(\mathrm{dd}, J=7.67,1.62 \mathrm{~Hz}, 1 \mathrm{H})$, $6.92(\mathrm{td}, J=7.51,1.08 \mathrm{~Hz}, 1 \mathrm{H}), 6.81(\mathrm{dd}, J=8.02,1.04 \mathrm{~Hz}, 1 \mathrm{H})$, 
$5.90(\mathrm{t}, J=4.27 \mathrm{~Hz}, 1 \mathrm{H}), 4.77(\mathrm{~d}, J=3.98 \mathrm{~Hz}, 2 \mathrm{H}), 1.22(\mathrm{~s}, 9 \mathrm{H}) .{ }^{13} \mathrm{C} \mathrm{NMR}(100 \mathrm{MHz}$, DMSO- $\left.d_{6}\right) \delta 177.1,154.5,129.8,129.2,122.8,121.4,121.0,115.7,114.2,64.6,39.0$, 27.3. HRMS-ESI: Found $[\mathrm{M}+\mathrm{Na}]^{+}=254.1159 ; \mathrm{C}_{14} \mathrm{H}_{17} \mathrm{NO}_{2} \mathrm{Na}$ requires 254.1151 .

General procedure for the synthesis of synthesis of enol-pivalates

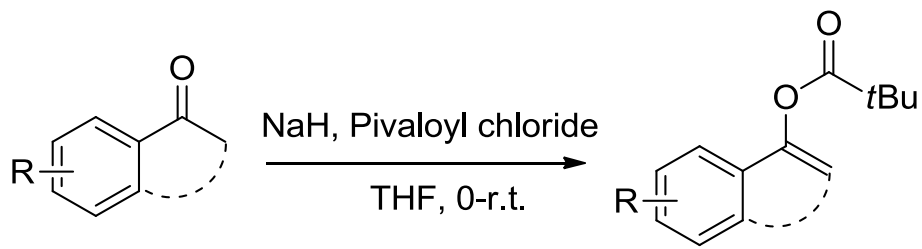

Enol-pivalates 1e, 1n, 1p, 1q, 1r were synthesized according to the reported procedures. $^{9}$

New compounds:

\section{$1 H$-inden-3-yl pivalate}

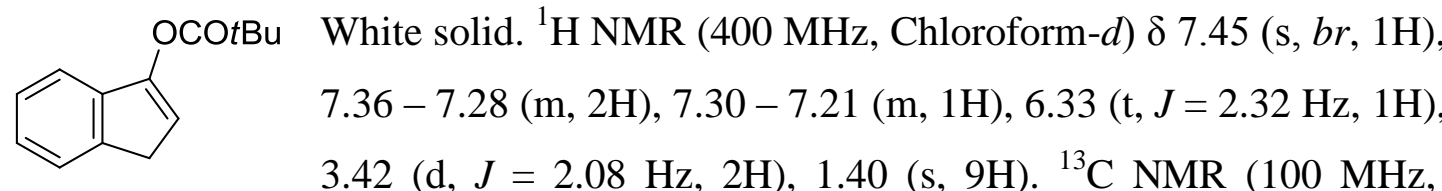

Chloroform- $d$ ) $\delta 175.9,149.3,141.9,139.5,126.4,125.8,124.3,118.0,115.3,39.6$, 35.2, 27.4. HRMS-ESI: Found $[\mathrm{M}+\mathrm{Na}]^{+}=239.1062 ; \mathrm{C}_{14} \mathrm{H}_{16} \mathrm{O}_{2} \mathrm{Na}$ requires 239.1043.

\section{3,4-dihydronaphthalen-1-yl pivalate}

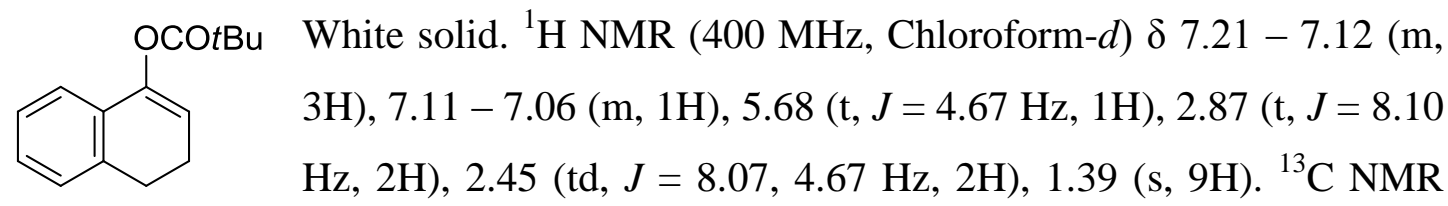
(100 MHz, Chloroform- $d$ ) $\delta 176.9,145.9,136.6,130.9,127.9,127.7,126.5,120.7$, 115.2, 39.3, 27.7, 27.4, 22.2. HRMS-ESI: Found $[\mathrm{M}+\mathrm{Na}]^{+}=253.1206 ; \mathrm{C}_{15} \mathrm{H}_{18} \mathrm{O}_{2} \mathrm{Na}$ requires 253.1199 .

\section{5-methyl-3,4-dihydronaphthalen-1-yl pivalate}<smiles>CCCCOC1=CCCc2c(C)cccc21</smiles>

White solid. ${ }^{1} \mathrm{H}$ NMR (400 MHz, Chloroform- $d$ ) $\delta 7.11-7.02(\mathrm{~m}$, $2 \mathrm{H}), 7.00-6.93(\mathrm{~m}, 1 \mathrm{H}), 5.65(\mathrm{t}, J=4.69 \mathrm{~Hz}, 1 \mathrm{H}), 2.81(\mathrm{t}, J=8.20$ Hz, 2H), 2.44 (ddd, $J=9.13,7.60,4.71 \mathrm{~Hz}, 2 \mathrm{H}), 2.28$ (s, 3H), 1.37 (s, 9H). ${ }^{13} \mathrm{C}$ NMR (100 MHz, Chloroform-d) $\delta$ 177.0, 146.0, 135.2, 
$134.9,130.7,130.1,125.8,118.7,114.6,39.3,27.5,23.6,21.8,19.8$. HRMS-ESI: Found $[\mathrm{M}+\mathrm{Na}]^{+}=267.1349 ; \mathrm{C}_{16} \mathrm{H}_{20} \mathrm{O}_{2} \mathrm{Na}$ requires 267.1356 .

\section{5-methoxy-3,4-dihydronaphthalen-1-yl pivalate}<smiles>CCCCOC(=O)C1=CCCc2c(OC)cccc21</smiles>

White solid. ${ }^{1} \mathrm{H}$ NMR (400 MHz, Chloroform- $d$ ) $\delta 7.12(\mathrm{t}, J=7.96$, $7.50 \mathrm{~Hz}, 1 \mathrm{H}), 6.80(\mathrm{dd}, J=8.31,1.06 \mathrm{~Hz}, 1 \mathrm{H}), 6.74(\mathrm{~d}, J=7.82 \mathrm{~Hz}$, $1 \mathrm{H}), 5.66(\mathrm{t}, J=4.67 \mathrm{~Hz}, 1 \mathrm{H}), 3.83(\mathrm{~s}, 3 \mathrm{H}), 2.86(\mathrm{t}, J=8.31 \mathrm{~Hz}$, $2 \mathrm{H}), 2.42$ (ddd, $J=9.36,7.71,4.69 \mathrm{~Hz}, 2 \mathrm{H}), 1.37$ (s, 9H). ${ }^{13} \mathrm{C} \mathrm{NMR}$

(100 MHz, Chloroform-d) $\delta$ 177.0, 156.3, 145.7, 132.0, 130.7, 126.7, 124.6, 115.4, 113.6, 110.7, 55.7, 39.3, 27.5, 21.7, 19.8. HRMS-ESI: Found $[\mathrm{M}+\mathrm{Na}]^{+}=283.1305$; $\mathrm{C}_{16} \mathrm{H}_{20} \mathrm{O}_{3} \mathrm{Na}$ requires 283.1305.

\section{7-methyl-3,4-dihydronaphthalen-1-yl pivalate}

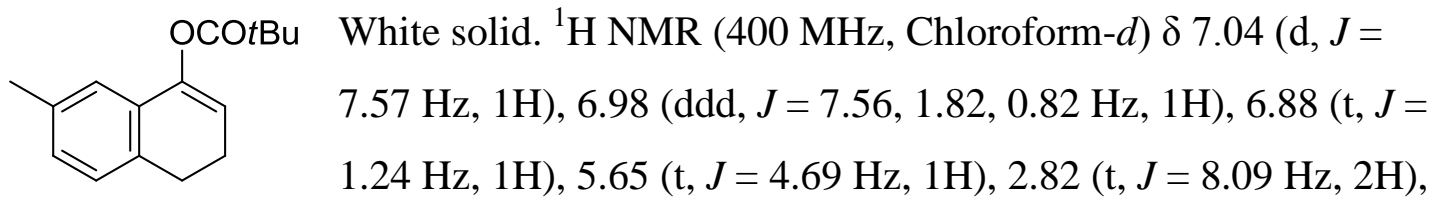
$2.47-2.39(\mathrm{~m}, 2 \mathrm{H}), 2.29$ (s, 3H), 1.38 (s, 9H). ${ }^{13} \mathrm{C}$ NMR (100 MHz, Chloroform-d) $\delta$ 177.0, 146.0, 135.9, 133.6, 130.7, 128.5, 127.6, 121.4, 115.2, 39.3, 27.5, 27.3, 22.4,

21.5. HRMS-ESI: Found $[\mathrm{M}+\mathrm{Na}]^{+}=267.1373 ; \mathrm{C}_{16} \mathrm{H}_{20} \mathrm{O}_{2} \mathrm{Na}$ requires 267.1356.

\section{General procedure for the synthesis of the other substrates}

Substrate $4 \mathbf{4 e},{ }^{16} \mathbf{4 h},{ }^{10} \mathbf{4} \mathbf{l},{ }^{11} \mathbf{4 m},{ }^{11} \mathbf{4 o},{ }^{12} \mathbf{4} \mathbf{p},{ }^{12} \mathbf{4 q}{ }^{13}$ were prepared according to the literature procedures, and the characterization data has been included.

Substrate $\mathbf{4 n}$ was prepared according to the literature procedure. ${ }^{13}$

Methyl (Z)-2-acetamido-3-(4-(tert-butyl)phenyl)acrylate

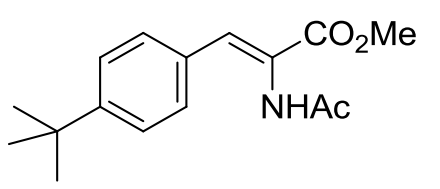

White solid. ${ }^{1} \mathrm{H}$ NMR (400 MHz, DMSO- $d_{6}$ ) $\delta 9.59$ (s, $b r$, $1 \mathrm{H}), 7.57(\mathrm{~d}, J=8.55 \mathrm{~Hz}, 2 \mathrm{H}), 7.44(\mathrm{~d}, J=8.51 \mathrm{~Hz}, 2 \mathrm{H})$, $7.16(\mathrm{~s}, 1 \mathrm{H}), 3.70(\mathrm{~s}, 3 \mathrm{H}), 1.99$ (s, 3H), $1.28(\mathrm{~s}, 9 \mathrm{H}) .{ }^{13} \mathrm{C}$ NMR (100 MHz, DMSO- $\left.d_{6}\right) \delta 169.3,165.6,152.2,131.1,130.6,129.7,126.0,125.4$, 52.1, 34.5, 30.9, 22.4. HRMS-ESI: Found $[\mathrm{M}+\mathrm{Na}]^{+}=298.1420 ; \mathrm{C}_{16} \mathrm{H}_{21} \mathrm{NO}_{3} \mathrm{Na}$ requires 298.1414 . 


\section{Development of arene hydrogenation based on $\mathrm{Rh} /$ diphosphine system and}

kinetic studies

Asymmetric olefin hydrogenation of $1 \mathrm{a}$ with homogeneous $\mathrm{Rh}$ catalyst $(\mathrm{Rh} / \mathrm{L}=1: 1)$.

To a dry $4 \mathrm{~mL}$ vial was charged with $\left[\mathrm{Rh}(\mathrm{COD})_{2}\right] \mathrm{BF}_{4}(2 \mathrm{~mol} \%)$ and diphosphine ligand (S,S)-Me-BPE (2 mol\%, $0.5 \mathrm{~mL}$ stock solution in $i$-PrOH) under argon. The mixture was stirred for $0.5 \mathrm{~h}$. The substrate $1 \mathrm{a}(0.1 \mathrm{mmol})$ was dissolved in $0.5 \mathrm{~mL} i$ $\mathrm{PrOH}$ and then added to the vial under argon. The vial was placed in a high-pressure hydrogenation apparatus. The reactor was purged three times with $\mathrm{Ar}$, and then filled with $\mathrm{H}_{2}$ (10 bar). The reaction was stirred at room temperature for $12 \mathrm{~h}$ before the $\mathrm{H}_{2}$ pressure was released and the solvent was removed in vacuo. The crude product was filtered through a short plug of silica with 1:1 $\mathrm{Et}_{2} \mathrm{O} / \mathrm{Pentane}$ as the eluent to yield product 2a.

\section{Complete hydrogenation of $1 \mathrm{a}$ with $\left[\mathrm{Rh}(\mathrm{COD})_{2}\right] \mathrm{BF}_{4}$.}

To a dry $4 \mathrm{~mL}$ vial was charged with $\left[\mathrm{Rh}(\mathrm{COD})_{2}\right] \mathrm{BF}_{4}(2 \mathrm{~mol} \%)$, substrate $\mathbf{1 a}(0.1$ mmol) and $1 \mathrm{~mL} i$-PrOH. The vial was placed in a high-pressure hydrogenation apparatus. The reactor was purged three times with Ar, and then filled with $\mathrm{H}_{2}(10$ bar). The reaction was stirred at room temperature for $12 \mathrm{~h}$ before the $\mathrm{H}_{2}$ pressure was released and the solvent was removed in vacuo. The crude product was filtered through a short plug of silica with $1: 1 \mathrm{Et}_{2} \mathrm{O} / \mathrm{Pentane}$ as the eluent to yield the racemic product 3a.

Asymmetric full saturation of 1a with homogeneous $\mathrm{Rh} /$ diphosphine and in-situ generated heterogeneous $\mathrm{Rh}$-nanoparticles $(\mathrm{Rh} / \mathrm{L}=2: 1)$.

To a dry $4 \mathrm{~mL}$ vial was charged with $\left[\mathrm{Rh}(\mathrm{COD})_{2}\right] \mathrm{BF}_{4}(4 \mathrm{~mol} \%)$ and diphosphine ligand (S,S)-Me-BPE (2 mol\%, $0.5 \mathrm{~mL}$ stock solution in $i$-PrOH) under argon. The mixture was stirred for $0.5 \mathrm{~h}$. The substrate $1 \mathrm{a}(0.1 \mathrm{mmol})$ was dissolved in $0.5 \mathrm{~mL} i$ $\mathrm{PrOH}$ and then added to the vial under argon. The vial was placed in a high-pressure hydrogenation apparatus. The reactor was purged three times with Ar, and then filled with $\mathrm{H}_{2}$ (10 bar). The reaction was stirred at room temperature for $12 \mathrm{~h}$ before the $\mathrm{H}_{2}$ pressure was released and the solvent was removed in vacuo. The crude product was 
filtered through a short plug of silica with $1: 1 \mathrm{Et}_{2} \mathrm{O} / \mathrm{Pentane}$ as the eluent to yield the enantio-enriched full reduced product $\mathbf{3 a}$.

\section{Kinetic studies of olefin hydrogenation of 1a.}

To a set of three $4 \mathrm{~mL}$ dry vials was charged with the corresponding amount of $\left[\mathrm{Rh}(\mathrm{COD})_{2}\right] \mathrm{BF}_{4}$ and diphosphine ligand $(\mathrm{S}, \mathrm{S})-\mathrm{Me}-\mathrm{BPE}(2 \mathrm{~mol} \%, 0.5 \mathrm{~mL}$ stock solution in $i$-PrOH) under argon. The mixture was stirred for $0.5 \mathrm{~h}$. The substrate $1 \mathrm{a}$ ( $0.1 \mathrm{mmol})$ was dissolved in $0.5 \mathrm{~mL} i$-PrOH and then added to the vial under argon. The vial was placed in a high-pressure hydrogenation apparatus. The reactor was purged three times with $\mathrm{Ar}$, and then filled with $\mathrm{H}_{2}$ (10 bar). The reaction was stirred at room temperature for the allocated time before the $\mathrm{H}_{2}$ pressure was released and the solvent was removed in vacuo. The distribution of products from the reaction mixture was determined by ${ }^{1} \mathrm{H}$ NMR spectroscopy.

\section{Kinetic studies of arene hydrogenation of $r a c-2 a$.}

To a set of three $4 \mathrm{~mL}$ dry vials was charged with the corresponding amount of $\left[\mathrm{Rh}(\mathrm{COD})_{2}\right] \mathrm{BF}_{4}$ and diphosphine ligand $(\mathrm{S}, \mathrm{S})-\mathrm{Me}-\mathrm{BPE}(2 \mathrm{~mol} \%, 0.5 \mathrm{~mL}$ stock solution in $i$-PrOH) under argon. The mixture was stirred for $0.5 \mathrm{~h}$. The substrate rac2a $(0.1 \mathrm{mmol})$ was dissolved in $0.5 \mathrm{~mL} i-\mathrm{PrOH}$ and then added to the vial under argon. The vial was placed in a high-pressure hydrogenation apparatus. The reactor was purged three times with $\mathrm{Ar}$, and then filled with $\mathrm{H}_{2}(10 \mathrm{bar})$. The reaction was stirred at room temperature for the allocated time before the $\mathrm{H}_{2}$ pressure was released and the solvent was removed in vacuo. The distribution of products from the reaction mixture was determined by ${ }^{1} \mathrm{H}$ NMR spectroscopy.

\section{Evaluation of Rh-precursors and the generality of diphosphine ligand.}

To a dry $4 \mathrm{~mL}$ vial was charged with the corresponding Rh-precursor and allocated amount of diphosphine ligand under argon. The mixture was stirred for $0.5 \mathrm{~h}$. The substrate $4 \mathbf{a}(0.05 \mathrm{mmol})$ was dissolved in $0.5 \mathrm{~mL} i$-PrOH and then added to the vial under argon. The vial was placed in a high-pressure hydrogenation apparatus. The reactor was purged three times with $\mathrm{Ar}$, and then filled with $\mathrm{H}_{2}(15 \mathrm{bar})$. The reaction was stirred at room temperature for $16 \mathrm{~h}$ before the $\mathrm{H}_{2}$ pressure was released and the solvent was removed in vacuo. The distribution of products from the reaction mixture 
was determined by ${ }^{1} \mathrm{H}$ NMR spectroscopy. Enantiomeric excess was determined by GC using a chiral stationary phase.

\section{General procedure for asymmetric hydrogenation of arenes}

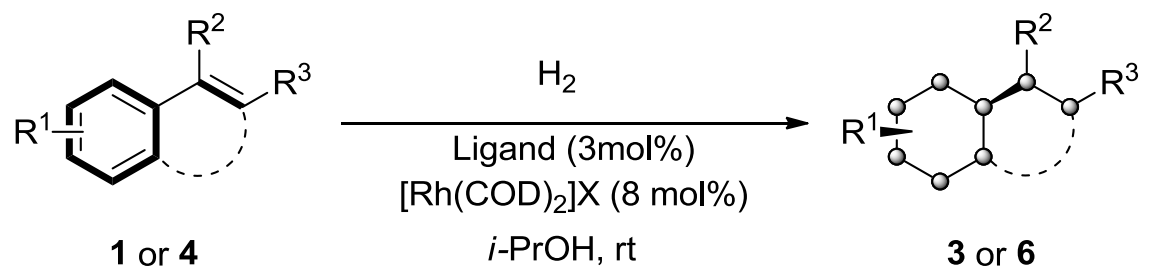

A vial was charged with Rh precursor ( 8 mol\%) and chiral ligand (3 mol\%) under Argon. Degassed dry $i \operatorname{PrOH}(2.0 \mathrm{ml})$ was added and the vial was stirred for $0.5 \mathrm{~h}$. The substrates $(0.2 \mathrm{mmol})$ were added under Argon (so that the concentration of the substrate was $0.1 \mathrm{M}$ ) and the vial was placed in a high-pressure hydrogenation apparatus. The reactor was purged three times with $\mathrm{Ar}$ gas, then filled with $\mathrm{H}_{2}$. The reaction was stirred at room temperature for $24 \mathrm{~h}$ before the $\mathrm{H}_{2}$ pressure was released and the solvent was removed in vacuo. The crude product was filtered through on a short plug of silica. Conversions were determined by ${ }^{1} \mathrm{H}$ NMR spectroscopy and $e e$ values were determined by GC using a chiral stationary phase.

Table S1. Characterization data for fully hydrogenated products.

\begin{tabular}{|c|c|c|c|c|c|}
\hline Entry & Product & d.r. & Separation method & $\begin{array}{l}\text { Optical } \\
\text { rotation }\end{array}$ & $\begin{array}{c}e e l \\
\%\end{array}$ \\
\hline 1 & & -- & $\begin{array}{l}\text { GC-MS: column } \\
\text { Chiraldex } \beta \text {-DM, } \\
50{ }^{\circ} \mathrm{C} \text { to } 175^{\circ} \mathrm{C} \text { at } \\
1{ }^{\circ} \mathrm{C} / \text { min. } \mathrm{t}_{\mathrm{R}}=89.6 \\
\text { min. }\end{array}$ & $\begin{array}{l}{[\alpha]_{\mathrm{D}}^{29}=} \\
-16.0(\mathrm{c}= \\
0.2, \\
\left.\mathrm{CHCl}_{3}\right)\end{array}$ & 99 \\
\hline 2 & & $76: 24$ & $\begin{array}{l}\text { GC-MS: column } \\
\text { Chiraldex } \beta \text {-DM, } \\
60{ }^{\circ} \mathrm{C} \text { iso } 180 \mathrm{~min} . \\
\mathrm{t}_{\mathrm{R}}=82.9 \mathrm{~min} / 85.1 \\
\text { min (major) }\end{array}$ & - & 99 \\
\hline 3 & & $47: 44: 5: 4$ & $\begin{array}{l}\text { GC-MS: column } \\
\text { Chiraldex } \beta \text {-DM, } \\
50{ }^{\circ} \mathrm{C} \text { to } 175^{\circ} \mathrm{C} \text { at } \\
1{ }^{\circ} \mathrm{C} / \mathrm{min} . \mathrm{t}_{\mathrm{R}}=40.4 \\
\mathrm{~min} / 41.7 \mathrm{~min} / 42.0 \\
\mathrm{~min} / 42.3 \mathrm{~min} .\end{array}$ & -- & 99 \\
\hline
\end{tabular}




\begin{tabular}{|c|c|c|c|c|c|}
\hline 4 & 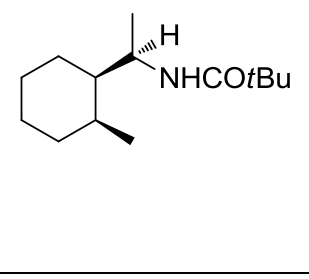 & $88: 12$ & $\begin{array}{l}\text { GC-MS: column } \\
\text { Chiraldex } \beta \text {-DM, } \\
50{ }^{\circ} \mathrm{C} \text { to } 175^{\circ} \mathrm{C} \text { at } \\
1{ }^{\circ} \mathrm{C} / \mathrm{min} . \mathrm{t}_{\mathrm{R}}=72.0 \\
\mathrm{~min} / 73.2 \mathrm{~min} / 73.6 \\
\mathrm{~min} / 74.7 \mathrm{~min} .\end{array}$ & -- & 89 \\
\hline 5 & & $86: 14$ & $\begin{array}{l}\text { GC-MS: column } \\
\text { Chiraldex } \beta-\mathrm{DM} \text {, } \\
50{ }^{\circ} \mathrm{C} \text { to } 175^{\circ} \mathrm{C} \text { at } \\
1{ }^{\circ} \mathrm{C} / \text { min. } t_{\mathrm{R}}=66.0 \\
\text { min (minor) } / 81.0 \\
\text { min (major) }\end{array}$ & -- & 99 \\
\hline 6 & & $82: 18$ & $\begin{array}{l}\text { GC-MS: column } \\
\text { Chiraldex } \beta \text {-DM, } \\
50{ }^{\circ} \mathrm{C} \text { to } 175^{\circ} \mathrm{C} \text { at } \\
1{ }^{\circ} \mathrm{C} / \mathrm{min} . \mathrm{t}_{\mathrm{R}}=66.7 \\
\mathrm{~min} / 67.8 \mathrm{~min} / 69.0 \\
\mathrm{~min}\end{array}$ & -- & 96 \\
\hline 7 & 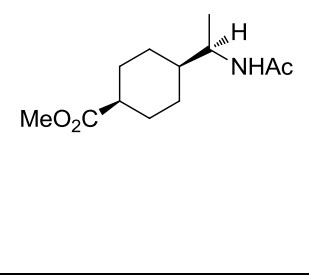 & $90: 10$ & $\begin{array}{l}\text { GC-MS: column } \\
\text { Chiraldex } \beta \text {-DM, } \\
120{ }^{\circ} \mathrm{C} \text { iso at } \\
1{ }^{\circ} \mathrm{C} / \mathrm{min} . \mathrm{t}_{\mathrm{R}}=32.1 \\
\mathrm{~min} / 33.2 \mathrm{~min} / 57.0 \\
\mathrm{~min}\end{array}$ & -- & 92 \\
\hline 8 & & $80: 20$ & $\begin{array}{l}\text { GC-MS: column } \\
\text { Chiraldex } \beta \text {-DM, } \\
50{ }^{\circ} \mathrm{C} \text { to } 175^{\circ} \mathrm{C} \text { at } \\
1{ }^{\circ} \mathrm{C} / \mathrm{min} . \mathrm{t}_{\mathrm{R}}=77.8 \\
\mathrm{~min} / 108.2 \mathrm{~min} .\end{array}$ & -- & 99 \\
\hline 9 & & $84: 16$ & $\begin{array}{l}\text { GC-MS: column } \\
\text { Chiraldex } \beta \text {-DM, } \\
50{ }^{\circ} \mathrm{C} \text { to } 175^{\circ} \mathrm{C} \text { at } \\
1{ }^{\circ} \mathrm{C} / \mathrm{min} . \mathrm{t}_{\mathrm{R}}=64.5 \\
\mathrm{~min} / 65.1 \mathrm{~min} / 66.9 \\
\mathrm{~min} / 66.3 \mathrm{~min} .\end{array}$ & -- & 98 \\
\hline 10 & & $55: 45$ & $\begin{array}{l}\text { GC-MS: column } \\
\text { Chiraldex } \beta-\mathrm{DM} \text {, } \\
50{ }^{\circ} \mathrm{C} \text { to } 175^{\circ} \mathrm{C} \text { at } \\
1{ }^{\circ} \mathrm{C} / \mathrm{min} . \mathrm{t}_{\mathrm{R}}=64.5 \\
\mathrm{~min} / 65.1 \mathrm{~min} / 66.9 \\
\mathrm{~min} / 66.3 \mathrm{~min} .\end{array}$ & -- & 98 \\
\hline 11 & & $82: 18$ & $\begin{array}{l}\text { GC-MS: column } \\
\text { Chiraldex } \beta \text {-DM, } \\
50{ }^{\circ} \mathrm{C} \text { to } 175^{\circ} \mathrm{C} \text { at } \\
1{ }^{\circ} \mathrm{C} / \mathrm{min} . \mathrm{t}_{\mathrm{R}}=71.6 \\
\mathrm{~min} / 72.8 \mathrm{~min} / 75.1 \\
\mathrm{~min} .\end{array}$ & -- & 96 \\
\hline
\end{tabular}




\begin{tabular}{|c|c|c|c|c|c|}
\hline 12 & $H^{A C}$ & $81: 19$ & $\begin{array}{l}\text { GC-MS: column } \\
\text { Chiraldex } \beta-\mathrm{DM} \text {, } \\
50{ }^{\circ} \mathrm{C} \text { to } 175^{\circ} \mathrm{C} \text { at } \\
1^{\circ} \mathrm{C} / \mathrm{min} . \mathrm{t}_{\mathrm{R}}=94.5 \\
\mathrm{~min} / 95.1 \mathrm{~min} / 96.7 \\
\mathrm{~min} \text {. }\end{array}$ & -- & 90 \\
\hline 13 & & $82: 18$ & $\begin{array}{l}\text { GC-MS: column } \\
\text { Chiraldex } \beta \text {-DM, } \\
120{ }^{\circ} \mathrm{C} \text { iso at } \\
1{ }^{\circ} \mathrm{C} / \mathrm{min} . \mathrm{t}_{\mathrm{R}}= \\
111.5 \mathrm{~min} /{ }_{1} 18.7 \\
\mathrm{~min} / 145.5 \mathrm{~min} .\end{array}$ & -- & 96 \\
\hline 14 & & $73: 27$ & $\begin{array}{l}\text { GC-MS: column } \\
\text { Chiraldex } \beta-\mathrm{DM} \text {, } \\
50{ }^{\circ} \mathrm{C} \text { to } 175^{\circ} \mathrm{C} \text { at } \\
1{ }^{\circ} \mathrm{C} / \mathrm{min} . \mathrm{t}_{\mathrm{R}}= \\
114.3 \mathrm{~min} / 117.4 \\
\text { min. }\end{array}$ & -- & 99 \\
\hline 15 & $\begin{array}{l}\mathrm{O}_{2} \mathrm{Me} \\
{ }_{\mathrm{C}}\end{array}$ & $83: 17$ & $\begin{array}{l}\text { GC-MS: column } \\
\text { Chiraldex } \beta \text {-DM, } \\
50{ }^{\circ} \mathrm{C} \text { to } 175^{\circ} \mathrm{C} \text { at } \\
1{ }^{\circ} \mathrm{C} / \mathrm{min} . \mathrm{t}_{\mathrm{R}}=92.5 \\
\text { min } / 94.6 \mathrm{~min} .\end{array}$ & -- & 99 \\
\hline 16 & & $81: 19$ & $\begin{array}{l}\text { GC-MS: column } \\
\text { Chiraldex } \beta-\mathrm{DM} \text {, } \\
50{ }^{\circ} \mathrm{C} \text { to } 175^{\circ} \mathrm{C} \text { at } \\
1{ }^{\circ} \mathrm{C} / \mathrm{min} . \mathrm{t}_{\mathrm{R}}=92.1 \\
\text { min / } 92.4 \text { min } \\
/ 93.6 \text { min. }\end{array}$ & -- & 96 \\
\hline 17 & & $85: 15$ & $\begin{array}{l}\text { GC-MS: column } \\
\text { Chiraldex } \beta-\mathrm{DM} \text {, } \\
50{ }^{\circ} \mathrm{C} \text { to } 175^{\circ} \mathrm{C} \text { at } \\
1{ }^{\circ} \mathrm{C} / \mathrm{min} . \mathrm{t}_{\mathrm{R}}=94.4 \\
\text { min } / 96.1 \mathrm{~min} .\end{array}$ & -- & 99 \\
\hline 18 & Ac & $66: 34$ & $\begin{array}{l}\text { GC-MS: column } \\
\text { Chiraldex } \beta \text {-DM, } \\
50{ }^{\circ} \mathrm{C} \text { to } 175^{\circ} \mathrm{C} \text { at } \\
1^{\circ} \mathrm{C} / \mathrm{min} . \mathrm{t}_{\mathrm{R}}=81.1 \\
\mathrm{~min} / 82.6 \mathrm{~min} / 83.7 \\
\mathrm{~min} / 85.3 \mathrm{~min} .\end{array}$ & -- & 96 \\
\hline 19 & OiPr & $76: 24$ & $\begin{array}{l}\text { GC-MS: column } \\
\text { Chiraldex } \beta \text {-DM, } \\
50{ }^{\circ} \mathrm{C} \text { to } 175^{\circ} \mathrm{C} \text { at } \\
1{ }^{\circ} \mathrm{C} / \mathrm{min} . \mathrm{t}_{\mathrm{R}}=59.8 \\
\text { min/ } 72.5 \mathrm{~min} .\end{array}$ & -- & 99 \\
\hline
\end{tabular}




\begin{tabular}{|c|c|c|c|c|c|}
\hline 20 & tBu & $82: 18$ & $\begin{array}{l}\text { GC-MS: column } \\
\text { Chiraldex } \beta \text {-DM, } \\
50{ }^{\circ} \mathrm{C} \text { to } 175^{\circ} \mathrm{C} \text { at } \\
1{ }^{\circ} \mathrm{C} / \mathrm{min} . \mathrm{t}_{\mathrm{R}}=83.0 \\
\text { min } / 87.9 \mathrm{~min} .\end{array}$ & -- & 99 \\
\hline 21 & $\mathrm{COCF}_{3}$ & $93: 7$ & $\begin{array}{l}\text { GC-MS: column } \\
\text { Chiraldex } \beta \text {-DM, } \\
50{ }^{\circ} \mathrm{C} \text { to } 175^{\circ} \mathrm{C} \text { at } \\
1{ }^{\circ} \mathrm{C} / \mathrm{min} . \mathrm{t}_{\mathrm{R}}=56.7 \\
\min / 58.2 \mathrm{~min} .\end{array}$ & $\begin{array}{l}{[\alpha]_{\mathrm{D}}{ }^{29}=} \\
76.5(\mathrm{c}= \\
0.2, \\
\left.\mathrm{CHCl}_{3}\right)\end{array}$ & 99 \\
\hline 22 & OtBu & $95: 5$ & $\begin{array}{l}\text { GC-MS: column } \\
\text { IVADEX-1, } 50{ }^{\circ} \mathrm{C} \\
\text { to } 175^{\circ} \mathrm{C} \text { at } \\
1{ }^{\circ} \mathrm{C} / \text { min. } \mathrm{t}_{\mathrm{R}}=73.2 \\
\text { min } / 79.1 \mathrm{~min} .\end{array}$ & $\begin{array}{l}{[\alpha]_{\mathrm{D}}{ }^{29}=} \\
17.5(\mathrm{c}= \\
0.2, \\
\left.\mathrm{CHCl}_{3}\right)\end{array}$ & 99 \\
\hline 23 & OtBu & $>99: 1$ & $\begin{array}{l}\text { GC-MS: column } \\
\text { Chiraldex } \beta \text {-DM, } \\
50{ }^{\circ} \mathrm{C} \text { to } 175^{\circ} \mathrm{C} \text { at } \\
1{ }^{\circ} \mathrm{C} / \text { min. } \mathrm{t}_{\mathrm{R}}=89.6 \\
\text { min. }\end{array}$ & $\begin{array}{l}{[\alpha]_{\mathrm{D}}^{29}=-} \\
4.0 \quad(\mathrm{c}= \\
0.2, \\
\left.\mathrm{CHCl}_{3}\right)\end{array}$ & 99 \\
\hline 24 & & 99:1 & $\begin{array}{l}\text { GC-MS: column } \\
\text { Chiraldex } \beta \text {-DM, } \\
50{ }^{\circ} \mathrm{C} \text { to } 175^{\circ} \mathrm{C} \text { at } \\
1{ }^{\circ} \mathrm{C} / \mathrm{min} . \mathrm{t}_{\mathrm{R}}=91.5 \\
\text { min } / 95.0 \mathrm{~min} .\end{array}$ & $\begin{array}{l}{[\alpha]_{\mathrm{D}}^{29}=} \\
-10.5(\mathrm{c}= \\
0.2, \\
\left.\mathrm{CHCl}_{3}\right)\end{array}$ & 99 \\
\hline 25 & $\mathrm{OCF}_{3}$ & $97: 3$ & $\begin{array}{l}\text { GC-MS: column } \\
\text { Chiraldex } \beta \text {-DM, } \\
50{ }^{\circ} \mathrm{C} \text { to } 175^{\circ} \mathrm{C} \text { at } \\
1{ }^{\circ} \mathrm{C} / \mathrm{min} . \mathrm{t}_{\mathrm{R}}=53.5 \\
\min / 55.6 \mathrm{~min} .\end{array}$ & $\begin{array}{l}{[\alpha]_{\mathrm{D}}^{29}=} \\
60.5 \quad(\mathrm{c}= \\
0.2, \\
\left.\mathrm{CHCl}_{3}\right)\end{array}$ & 99 \\
\hline 26 & & $97: 3$ & $\begin{array}{l}\text { GC-MS: column } \\
\text { Chiraldex } \beta \text {-DM, } \\
50{ }^{\circ} \mathrm{C} \text { to } 175^{\circ} \mathrm{C} \text { at } \\
1{ }^{\circ} \mathrm{C} / \text { min. } \mathrm{t}_{\mathrm{R}}=57.5 \\
\text { min / } 60.6 \mathrm{~min} / \\
63.5 \text { min. }\end{array}$ & $\begin{array}{l}{[\alpha]_{\mathrm{D}}{ }^{29}=} \\
72.5 \quad(\mathrm{c}= \\
0.2, \\
\left.\mathrm{CHCl}_{3}\right)\end{array}$ & 99 \\
\hline 27 & $\mathrm{HCOCF}_{3}$ & $95: 5$ & $\begin{array}{l}\text { GC-MS: column } \\
\text { Chiraldex } \beta \text {-DM, } \\
50{ }^{\circ} \mathrm{C} \text { to } 175^{\circ} \mathrm{C} \text { at } \\
1{ }^{\circ} \mathrm{C} / \mathrm{min} . \mathrm{t}_{\mathrm{R}}=71.7 \\
\text { min } / 74.5 \mathrm{~min} .\end{array}$ & $\begin{array}{l}{[\alpha]_{\mathrm{D}}{ }^{29}=} \\
20.5 \quad(\mathrm{c}= \\
0.2, \\
\left.\mathrm{CHCl}_{3}\right)\end{array}$ & 99 \\
\hline 28 & $\mathrm{H} \stackrel{\mathrm{NHCOCF}}{3}$ & $90: 6: 2: 2$ & $\begin{array}{l}\text { GC-MS: column } \\
\text { Chiraldex } \beta \text {-DM, } \\
50{ }^{\circ} \mathrm{C} \text { to } 175^{\circ} \mathrm{C} \text { at } \\
1{ }^{\circ} \mathrm{C} / \mathrm{min} . \mathrm{t}_{\mathrm{R}}=65.3 \\
\mathrm{~min} / 67.5 \mathrm{~min} / \\
69.9 \mathrm{~min} / 71.0 \mathrm{~min}\end{array}$ & $\begin{array}{l}{[\alpha]_{\mathrm{D}}^{29}=} \\
68.0 \quad(\mathrm{c}= \\
0.2, \\
\left.\mathrm{CHCl}_{3}\right)\end{array}$ & 99 \\
\hline
\end{tabular}




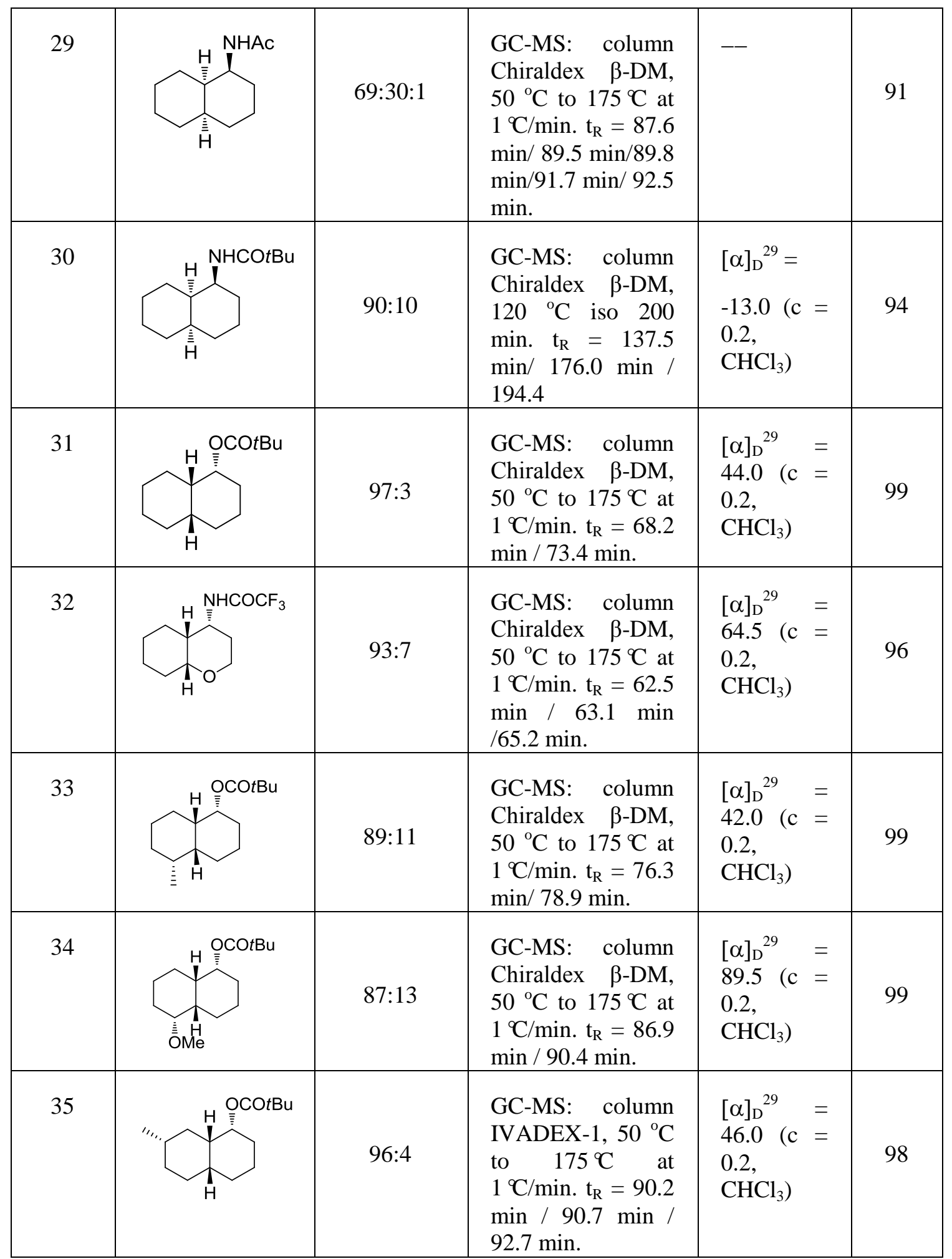




\section{Characterization of hydrogenated products}

\section{Methyl (R)-2-acetamido-3-cyclohexylpropanoate}

$\begin{array}{ll}\underbrace{\stackrel{\mathrm{N}_{\mathrm{HAc}}}{\mathrm{CO}_{2} \mathrm{Me}}}_{\mathrm{N}} & \text { White solid. }{ }^{1} \mathrm{H} \text { NMR }(400 \mathrm{MHz}, \text { Chloroform- } d) \delta 5.85(\mathrm{~d}, J= \\ 8.36 \mathrm{~Hz}, 1 \mathrm{H}), 4.65(\mathrm{td}, J=8.69,5.34 \mathrm{~Hz}, 1 \mathrm{H}), 3.73(\mathrm{~s}, 3 \mathrm{H}), 2.02\end{array}$ (s, 3H), $1.78(\mathrm{dtd}, J=13.06,3.84,2.26 \mathrm{~Hz}, 1 \mathrm{H}), 1.72-1.61(\mathrm{~m}, 5 \mathrm{H}), 1.50$ (ddd, $J=$ 13.90, 8.95, 5.76 Hz, 1H), 1.31 (dddq, $J=14.02,8.19,5.55,2.76,2.27 \mathrm{~Hz}, 1 \mathrm{H}$ ), 1.25 - $1.08(\mathrm{~m}, 3 \mathrm{H}), 1.01-0.82(\mathrm{~m}, 2 \mathrm{H}) .{ }^{13} \mathrm{C}$ NMR (100 MHz, Chloroform- $d$ ) $\delta 173.9$, $169.9,52.4,50.3,40.4,34.3,33.6,32.7,26.5,26.3,26.1,23.3 .[\alpha]_{\mathrm{D}}^{29}=16.0(\mathrm{c}=0.2$, $\left.\mathrm{CHCl}_{3}\right)$. HRMS-ESI: Found $[\mathrm{M}+\mathrm{Na}]^{+}=250.1424 ; \mathrm{C}_{12} \mathrm{H}_{21} \mathrm{NO}_{4} \mathrm{Na}$ requires 250.1414.

\section{2,2,2-trifluoro- $N-((S)-1-(4-m e t h y l c y c l o h e x y l) e t h y l)$ acetamide}

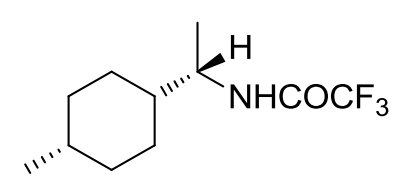

Colorless oil. ${ }^{1} \mathrm{H}$ NMR (400 MHz, Chloroform- $d$ ) major diastereomer: $\delta 6.11(\mathrm{~s}, b r, 1 \mathrm{H}), 4.03(\mathrm{dq}, J=13.47,6.76 \mathrm{~Hz}$, $1 \mathrm{H}), 1.77-1.59(\mathrm{~m}, 2 \mathrm{H}), 1.55-1.24(\mathrm{~m}, 8 \mathrm{H}), 1.19(\mathrm{~d}, J=$ $6.65 \mathrm{~Hz}, 3 \mathrm{H}), 0.91(\mathrm{~d}, J=6.98 \mathrm{~Hz}, 3 \mathrm{H})$. Minor diastereomer: $\delta 6.11(\mathrm{~s}, b r, 1 \mathrm{H}), 3.88$ $(\mathrm{dq}, J=13.47,6.76 \mathrm{~Hz}, 1 \mathrm{H}), 1.77-1.59(\mathrm{~m}, 2 \mathrm{H}), 1.55-1.24(\mathrm{~m}, 8 \mathrm{H}), 1.17(\mathrm{~d}, J=$ $6.64 \mathrm{~Hz}, 3 \mathrm{H}), 0.87$ (d, $J=6.53 \mathrm{~Hz}, 3 \mathrm{H}) .{ }^{13} \mathrm{C}$ NMR (100 MHz, Chloroform- $d$ ) major diastereomer: $\delta 156.7(\mathrm{q}, J=36.36 \mathrm{~Hz}, 2 \mathrm{C}), 116.1(\mathrm{q}, J=288.21 \mathrm{~Hz} 2 \mathrm{C}), 48.9,41.7$, 30.8, 30.8, 29.1 24.8, 24.7, 19.3, 18.2. Minor diastereomer: $\delta 156.7$ (q, $J=36.36 \mathrm{~Hz}$, 2C), 116.1 (q, $J=288.21 \mathrm{~Hz} 2 \mathrm{C}$ ), 50.8, 42.5, 34.8, 34.8, 32.6, 29.0, 28.9, 22.6, 17.8. ${ }^{19} \mathrm{~F}$ NMR (377 MHz, Chloroform- $d$ ) major diastereomer: $\delta$-75.90. Minor diastereomer: $\delta$-75.91. HRMS-ESI: Found $[\mathrm{M}+\mathrm{Na}]^{+}=206.1234 ; \mathrm{C}_{11} \mathrm{H}_{18} \mathrm{~F}_{3} \mathrm{ONNa}$ requires 206.1233.

\section{1-(((R)-1-((1s,4S)-4-methoxycyclohexyl)ethyl)-12-azaneyl) ethan-1-one}

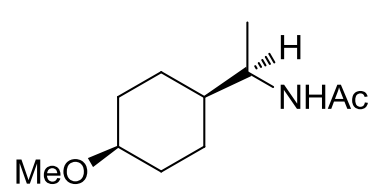

Colorless oil. ${ }^{1} \mathrm{H}$ NMR (400 MHz, Chloroform- $d$ ) major diastereomer: $\delta 5.63(\mathrm{~s}, b r, 1 \mathrm{H}), 3.91(\mathrm{dq}, J=13.95,6.86 \mathrm{~Hz}$, $1 \mathrm{H}), 3.45-3.40(\mathrm{~m}, 1 \mathrm{H}), 3.28(\mathrm{~s}, 3 \mathrm{H}), 2.15-1.71(\mathrm{~m}, 5 \mathrm{H})$, $1.52-1.21(\mathrm{~m}, 6 \mathrm{H}), 1.20-0.99(\mathrm{~m}, 1 \mathrm{H}), 1.09(\mathrm{~d}, J=6.80 \mathrm{~Hz}, 2 \mathrm{H})$. Minor diastereomer: $\delta 5.52(\mathrm{~s}, b r, 1 \mathrm{H}), 3.91(\mathrm{dq}, J=13.95,6.86 \mathrm{~Hz}, 1 \mathrm{H}), 3.34(\mathrm{~s}, 1 \mathrm{H}), 3.11$ $3.02(\mathrm{~m}, 1 \mathrm{H}), 2.15-1.71(\mathrm{~m}, 5 \mathrm{H}), 1.52-1.21(\mathrm{~m}, 6 \mathrm{H}), 1.20-0.99(\mathrm{~m}, 1 \mathrm{H}),{ }^{13} \mathrm{C}$ NMR (101 MHz, $\mathrm{CDCl}_{3}$ ) major diastereomer: $\delta$ 169.8, 74.7, 55.6, 53.4, 49.4, 42.1, 31.4, 29.1, 29.0, 22.8, 17.7. Minor diastereomer: $\delta$ 169.7, 79.3, 55.7, 49.3, 49.2, 42.5, 
27.2, 27.1, 23.3, 23.3, 18.3. HRMS-ESI: Found $[\mathrm{M}+\mathrm{Na}]^{+}=221.1358 ; \mathrm{C}_{11} \mathrm{H}_{20} \mathrm{NO}_{2} \mathrm{Na}$ requires 221.1386 .

\section{1-(((R)-1-((1s,4S)-4-methylcyclohexyl)ethyl)-12-azaneyl)ethan-1-one}

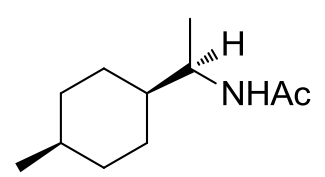

Colorless oil. ${ }^{1} \mathrm{H}$ NMR (400 MHz, Chloroform- $d$ ) major diastereomer: $\delta 5.25(\mathrm{~s}, b r, 1 \mathrm{H}), 3.99(\mathrm{dq}, J=13.56,6.88 \mathrm{~Hz}$, $1 \mathrm{H}), 1.97(\mathrm{~s}, 3 \mathrm{H}), 1.83-1.61(\mathrm{~m}, 2 \mathrm{H}), 1.54-1.15(\mathrm{~m}, 8 \mathrm{H}), 1.09$ $(\mathrm{d}, J=6.72 \mathrm{~Hz}, 3 \mathrm{H}), 0.91(\mathrm{~d}, J=6.98 \mathrm{~Hz}, 3 \mathrm{H})$. Minor diastereomer: $\delta 5.25(\mathrm{~s}, b r, 1 \mathrm{H})$, $3.85(\mathrm{dq}, J=13.42,6.83 \mathrm{~Hz}, 1 \mathrm{H}), 1.97(\mathrm{~s}, 3 \mathrm{H}), 1.83-1.61(\mathrm{~m}, 2 \mathrm{H}), 1.54-1.15(\mathrm{~m}$, $8 \mathrm{H}), 1.08(\mathrm{~d}, J=6.76 \mathrm{~Hz}, 3 \mathrm{H}), 0.86(\mathrm{~d}, J=6.54 \mathrm{~Hz}, 3 \mathrm{H}) .{ }^{13} \mathrm{C}$ NMR $(100 \mathrm{MHz}$, Chloroform- $d$ ) major diastereomer: $\delta$ 169.6, 47.8, 41.9, 34.9, 30.9, 30.8, 29.1, 24.8, 24.7, 19.3, 18.5. Minor diastereomer: $\delta$ 169.6, 49.7, 42.7, 32.6, 29.7, 29.0, 28.8, 23.4, 23.3, 22.5, 18.0. HRMS-ESI: Found $[\mathrm{M}+\mathrm{Na}]^{+}=205.1460 ; \mathrm{C}_{11} \mathrm{H}_{20} \mathrm{NONa}$ requires 205.1437 .

\section{$(1 S, 4 s)$-methyl 4-((R)-1-acetamidoethyl)cyclohexanecarboxylate}

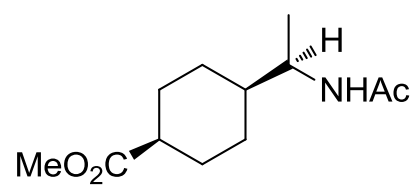

Colorless oil. ${ }^{1} \mathrm{H}$ NMR (400 MHz, Chloroform- $d$ ) major diastereomer: $\delta 5.33(\mathrm{~d}, J=9.6 \mathrm{~Hz}, 1 \mathrm{H}), 3.94-3.86(\mathrm{~m}$, $1 \mathrm{H}), 3.65(\mathrm{~s}, 3 \mathrm{H}), 2.62-2.53(\mathrm{~m}, 1 \mathrm{H}), 2.15-2.03(\mathrm{~m}, 2 \mathrm{H})$, $1.94(\mathrm{~s}, 3 \mathrm{H}), 1.62-1.43(\mathrm{~m}, 4 \mathrm{H}), 1.39-1.21(\mathrm{~m}, 3 \mathrm{H}), 1.05(\mathrm{~d}, J=6.8 \mathrm{~Hz}, 3 \mathrm{H})$. Minor diastereomer: $\delta 5.37(\mathrm{~d}, J=9.6 \mathrm{~Hz}, 1 \mathrm{H}), 3.91-3.80(\mathrm{~m}, 1 \mathrm{H}), 3.64(\mathrm{~s}, 3 \mathrm{H})$, $2.65-2.60(\mathrm{~m}, 1 \mathrm{H}), 2.26-2.16(\mathrm{~m}, 2 \mathrm{H}), 1.96(\mathrm{~s}, 3 \mathrm{H}), 1.62-1.43(\mathrm{~m}, 4 \mathrm{H}), 1.39-$ $1.21(\mathrm{~m}, 3 \mathrm{H}), 1.07$ (d, $J=6.8 \mathrm{~Hz}, 3 \mathrm{H}) .{ }^{13} \mathrm{C}$ NMR (100 MHz, Chloroform- $d$ ) major diastereomer: $\delta$ 175.6, 169.4, 51.6, 48.4, 42.1, 39.7, 26.8, 26.6, 25.7, 25.4, 23.7, 18.1 . Minor diastereomer: $\delta 176.4,169.4,51.7,49.2,43.2,42.5,28.8,28.2,28.2,26.7,25.5$, 18.2. HRMS-ESI: Found $[\mathrm{M}+\mathrm{Na}]^{+}=250.1426$.; $\mathrm{C}_{15} \mathrm{H}_{21} \mathrm{NO}_{2} \mathrm{Na}$ requires 250.1414 .

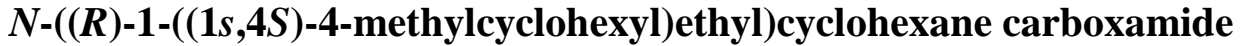

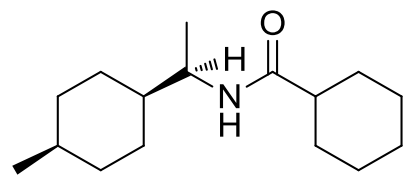

White solid. ${ }^{1} \mathrm{H}$ NMR (400 MHz, Chloroform- $d$ ) major diastereomer: $\delta 5.37(\mathrm{~s}, b r, 1 \mathrm{H}), 3.98(\mathrm{dq}, J=14.30,6.89$ $\mathrm{Hz}, 1 \mathrm{H}), 3.84(\mathrm{dq}, J=13.55,6.80 \mathrm{~Hz}, 1 \mathrm{H}), 2.07$ (ddt, $J=$ $11.72,7.09,3.39 \mathrm{~Hz}, 1 \mathrm{H}), 1.89-1.62(\mathrm{~m}, 6 \mathrm{H}), 1.54-1.16(\mathrm{~m}, 14 \mathrm{H}), 1.08(\mathrm{~d}, J=$ $6.71 \mathrm{~Hz}, 3 \mathrm{H}), 0.90(\mathrm{~d}, J=6.97 \mathrm{~Hz}, 3 \mathrm{H})$. Minor diastereomer: $\delta 5.37(\mathrm{~s}, b r, 1 \mathrm{H}), 3.84$ $(\mathrm{dq}, J=13.55,6.80 \mathrm{~Hz}, 1 \mathrm{H}), 2.07(\mathrm{ddt}, J=11.72,7.09,3.39 \mathrm{~Hz}, 1 \mathrm{H}), 1.89-1.62(\mathrm{~m}$, 
$6 \mathrm{H}), 1.54-1.16(\mathrm{~m}, 14 \mathrm{H}), 1.05(\mathrm{~d}, J=6.71 \mathrm{~Hz}, 3 \mathrm{H}), 0.85(\mathrm{~d}, J=6.53 \mathrm{~Hz}, 3 \mathrm{H}) .{ }^{13} \mathrm{C}$ NMR (100 MHz, Chloroform- $d$ ) major diastereomer: $\delta$ 175.6, 47.2, 46.9, 42.2, 31.1, 31.0, 30.1, 29.7, 29.2, 25.9, 25.9, 25.9, 25.0, 24.8, 19.4, 18.8. Minor diastereomer: $\delta$ 175.6, 49.1, 43.0, 35.1, 35.0, 32.8, 30.1, 29.8, 29.1, 29.0, 28.9, 25.9, 25.9, 25.9, 22.7, 18.3. HRMS-ESI: Found $[\mathrm{M}+\mathrm{Na}]^{+}=274.2153 ; \mathrm{C}_{16} \mathrm{H}_{29} \mathrm{NONa}$ requires 274.2141 .

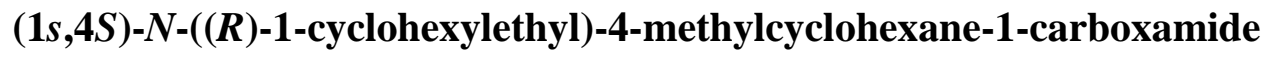

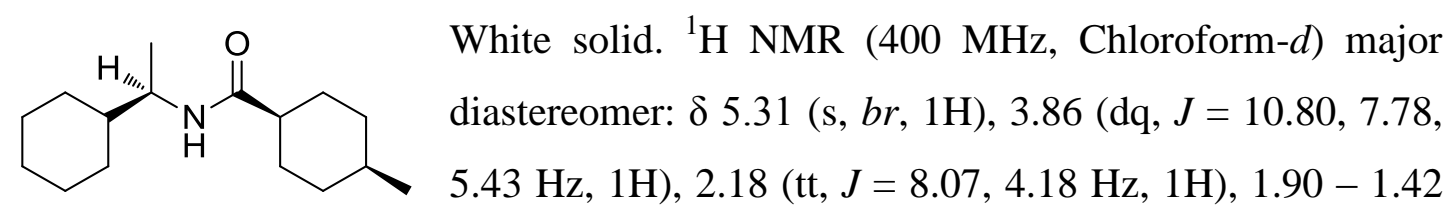
(m, 12H), $1.42-1.07(\mathrm{~m}, 6 \mathrm{H}), 1.05(\mathrm{~d}, J=6.77 \mathrm{~Hz}, 3 \mathrm{H}), 0.93(\mathrm{~d}, J=6.91 \mathrm{~Hz}, 3 \mathrm{H})$, $1.00-0.88(\mathrm{~m}, 2 \mathrm{H})$. Minor diastereomer: $\delta 5.28(\mathrm{~s}, b r, 1 \mathrm{H}), 3.86(\mathrm{dq}, J=10.80,5.43$ $\mathrm{Hz}, 1 \mathrm{H}), 1.96$ (tt, $J=12.13,3.49 \mathrm{~Hz}, 1 \mathrm{H}), 1.90-1.42(\mathrm{~m}, 12 \mathrm{H}), 1.42-1.07(\mathrm{~m}, 6 \mathrm{H})$, $1.04(\mathrm{~d}, J=6.76 \mathrm{~Hz}, 3 \mathrm{H}), 1.00-0.88(\mathrm{~m}, 2 \mathrm{H}), 0.87(\mathrm{~d}, J=6.54 \mathrm{~Hz}, 3 \mathrm{H}) .{ }^{13} \mathrm{C} \mathrm{NMR}$ (100 MHz, Chloroform- $d$ ) major diastereomer: $\delta 174.8,49.0,43.3,43.2,31.4,31.3$, 29.3, 29.3, 29.1, 26.6, 26.3, 26.3, 26.1, 25.8, 19.9, 18.2. Minor diastereomer: $\delta$ 175.5, 49.0, 45.8, 43.3, 34.7, 34.6, 32.1, 30.1, 29.8, 29.3, 29.1, 26.6, 26.5, 26.3, 22.7, 18.2. HRMS-ESI: Found $[\mathrm{M}+\mathrm{Na}]^{+}=274.2161 ; \mathrm{C}_{16} \mathrm{H}_{29} \mathrm{NONa}$ requires 274.2141.

\section{(S)-2-((R)-1-cyclohexylethyl)piperidine}

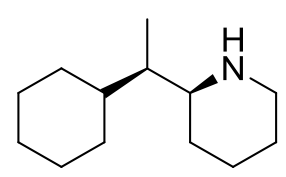

Colorless oil. ${ }^{1} \mathrm{H}$ NMR (400 MHz, Chloroform- $d$ ) major diastereomer: $\delta 3.58-3.50(\mathrm{~m}, 1 \mathrm{H}), 3.03-2.93(\mathrm{~m}, 1 \mathrm{H}), 2.94-$ $2.74(\mathrm{~m}, 1 \mathrm{H}), 1.94-1.60(\mathrm{~m}, 8 \mathrm{H}), 1.57-1.37(\mathrm{~m}, 4 \mathrm{H}), 1.33-1.04$ $(\mathrm{m}, 6 \mathrm{H}), 0.93(\mathrm{~d}, J=6.95 \mathrm{~Hz}, 3 \mathrm{H})$. Minor diastereomer: $\delta 3.58-3.50(\mathrm{~m}, 1 \mathrm{H}), 2.94-$ $2.74(\mathrm{~m}, 2 \mathrm{H}), 1.94-1.60(\mathrm{~m}, 8 \mathrm{H}), 1.57-1.37(\mathrm{~m}, 4 \mathrm{H}), 1.33-1.04(\mathrm{~m}, 6 \mathrm{H}), 1.00(\mathrm{~d}$, $J=6.89 \mathrm{~Hz}, 3 \mathrm{H}) .{ }^{13} \mathrm{C}$ NMR $(100 \mathrm{MHz}$, Chloroform- $d$ ) major diastereomer: $\delta 59.7$, 46.6, 40.9, 39.3, 32.1, 31.2, 29.4, 27.4, 26.7, 26.5, 24.8, 23.2, 11.3. Minor diastereomer: $\delta 60.5,46.5,41.5,35.8,32.0,29.8,28.0,26.8,26.4,25.9,24.8,11.3$. HRMS-ESI: Found $[\mathrm{M}+\mathrm{Na}]^{+}=218.1854 ; \mathrm{C}_{13} \mathrm{H}_{25} \mathrm{NNa}$ requires 218.1879. 


\section{2,2,2-trifluoro- $N-((S)-1-((1 S, 3 R)-3-$ methylcyclohexyl)ethyl) acetamide}

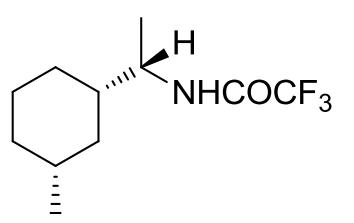

Colorless oil. ${ }^{1} \mathrm{H}$ NMR (400 MHz, Chloroform- $d$ ) major diastereomer: $\delta 6.09(\mathrm{~s}, b r, 1 \mathrm{H}), 3.88(\mathrm{dq}, J=15.11,7.40 \mathrm{~Hz}$, $1 \mathrm{H}), 1.86-1.21(\mathrm{~m}, 8 \mathrm{H}), 1.17(\mathrm{~d}, J=6.79 \mathrm{~Hz}, 3 \mathrm{H}), 0.90(\mathrm{~d}, J=$ $6.58 \mathrm{~Hz}, 3 \mathrm{H}), 0.88-0.53(\mathrm{~m}, 2 \mathrm{H})$. Minor diastereomer: $\delta 6.09$ (s, $b r, 1 \mathrm{H}), 4.03(\mathrm{dq}, J=15.11,7.40 \mathrm{~Hz}, 1 \mathrm{H}), 1.86-1.21(\mathrm{~m}, 8 \mathrm{H}), 1.17(\mathrm{~d}, J=6.79$ $\mathrm{Hz}, 3 \mathrm{H}), 0.90(\mathrm{~d}, J=6.58 \mathrm{~Hz}, 3 \mathrm{H}), 0.88-0.53(\mathrm{~m}, 2 \mathrm{H}) .{ }^{13} \mathrm{C}$ NMR $(100 \mathrm{MHz}$, Chloroform- $d$ ) major diastereomers: $\delta 156.6(\mathrm{q}, J=36.48), 118.3(\mathrm{q}, J=262.60 \mathrm{~Hz})$, $50.8,42.7,37.5,35.0,32.6,28.5,25.9,22.9,17.6$. Minor diastereomer: $\delta 156.6$ (q, $J=$ 36.48 ), 118.3 (q, $J=262.60 \mathrm{~Hz}$ ), 50.8, 42.8, 37.7, 35.0, 32.6, 28.5, 25.9, 22.9, 17.6. ${ }^{19}$ F NMR (377 MHz, Chloroform- $d$ ) major diastereomer: $\delta$-75.90, -75.91. HRMSESI: Found $[\mathrm{M}+\mathrm{Na}]^{+}=206.1129 ; \mathrm{C}_{11} \mathrm{H}_{18} \mathrm{~F}_{3} \mathrm{ONNa}$ requires 206.1233 .

\section{$N-((R)-1-((1 R, 2 S)-2-m e t h y l c y c l o h e x y l) e t h y l) p i v a l a m i d e$}

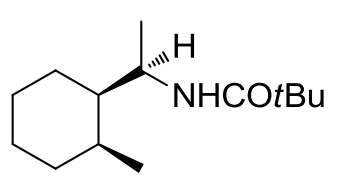

White solid. ${ }^{1} \mathrm{H}$ NMR (400 MHz, Chloroform- $d$ ) major diastereomer: $\delta 5.29(\mathrm{~s}, b r, 1 \mathrm{H}), 3.78(\mathrm{dq}, J=13.95,6.86 \mathrm{~Hz}$, $1 \mathrm{H}), 2.04-1.91(\mathrm{~m}, 1 \mathrm{H}), 1.75-1.67(\mathrm{~m}, 1 \mathrm{H}), 1.60-1.52(\mathrm{~m}$, $1 \mathrm{H}), 1.51-1.30(\mathrm{~m}, 4 \mathrm{H}), 1.27-1.12(\mathrm{~m}, 12 \mathrm{H}), 1.07(\mathrm{~d}, J=6.52 \mathrm{~Hz}, 3 \mathrm{H}), 0.86(\mathrm{~d}, J=$ $7.15 \mathrm{~Hz}, 3 \mathrm{H})$. Minor diastereomer: $\delta 5.36(\mathrm{~s}, \mathrm{br}, 1 \mathrm{H}), 4.31(\mathrm{dq}, J=13.95,6.86 \mathrm{~Hz}$, $1 \mathrm{H}), 2.04-1.91(\mathrm{~m}, 1 \mathrm{H}), 1.75-1.67(\mathrm{~m}, 1 \mathrm{H}), 1.60-1.52(\mathrm{~m}, 1 \mathrm{H}), 1.51-1.30(\mathrm{~m}$, 4H), $1.27-1.12(\mathrm{~m}, 12 \mathrm{H}), 1.07(\mathrm{~d}, J=6.52 \mathrm{~Hz}, 3 \mathrm{H}), 0.82(\mathrm{~d}, J=7.12 \mathrm{~Hz}, 3 \mathrm{H}) .{ }^{13} \mathrm{C}$ NMR (100 MHz, Chloroform- $d$ ) major diastereomer: $\delta$ 177.6, 47.6, 47.1, 38.8, 33.9, 29.4, 27.8, 26.8, 23.4, 20.3, 19.2, 12.0. Minor diastereomer: $\delta$ 177.4, 47.0, 46.4, 38.8, 33.7, 29.2, 27.8, 26.7, 24.0, 20.2, 20.1, 12.6. HRMS-ESI: Found $[\mathrm{M}+\mathrm{Na}]^{+}=$ 248.1990; $\mathrm{C}_{14} \mathrm{H}_{27} \mathrm{NONa}$ requires 248.1985.

\section{$N-((S)-1-((1 s, 4 R)-4-m e t h y l c y c l o h e x y l) p r o p y l)$ acetamide}

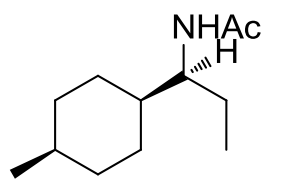

Colorless oil. ${ }^{1} \mathrm{H}$ NMR (400 MHz, Chloroform- $d$ ) major diastereomer: $\delta 5.36(\mathrm{~s}, b r, 1 \mathrm{H}), 3.86(\mathrm{qd}, J=9.73,5.03 \mathrm{~Hz}, 1 \mathrm{H})$, $2.01(\mathrm{~s}, 3 \mathrm{H}), 1.77-1.57(\mathrm{~m}, 3 \mathrm{H}), 1.52-1.20(\mathrm{~m}, 9 \mathrm{H}), 0.92-0.82$

(m, 6H). Minor diastereomer: $\delta 5.36(\mathrm{~s}, b r, 1 \mathrm{H}), 3.70(\mathrm{qd}, J=9.73,5.03 \mathrm{~Hz}, 1 \mathrm{H}), 2.00$ (s, 3H), $1.77-1.57(\mathrm{~m}, 3 \mathrm{H}), 1.52-1.20(\mathrm{~m}, 9 \mathrm{H}), 0.92-0.82(\mathrm{~m}, 6 \mathrm{H}) .{ }^{13} \mathrm{C}$ NMR (100 MHz, Chloroform- $d$ ) major diastereomer: $\delta$ 170.2, 53.3, 40.4, 31.2, 31.1, 29.1, 25.2, 
25.0, 24.5, 23.5, 19.3, 10.2. Minor diastereomer: $\delta$ 170.2, 55.4, 41.4, 35.1, 35.1, 32.8, 29.7, 28.5, 25.1, 23.5, 22.7, 10.6. HRMS-ESI: Found $[\mathrm{M}+\mathrm{Na}]^{+}=220.1660$; $\mathrm{C}_{12} \mathrm{H}_{23} \mathrm{NONa}$ requires 220.1672 .

\section{Methyl $(S)-3$-acetamido-3-((1s,4R)-4-methylcyclohexyl) propanoate}

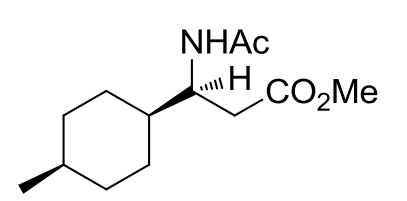

Colorless oil. ${ }^{1} \mathrm{H}$ NMR (400 MHz, Chloroform- $d$ ) major diastereomer: $\delta 6.13(\mathrm{~s}, b r, 1 \mathrm{H}), 4.21(\mathrm{ddt}, J=9.76,8.24$, $5.30 \mathrm{~Hz}, 1 \mathrm{H}), 3.66(\mathrm{~s}, 3 \mathrm{H}), 2.55(\mathrm{~d}, J=5.08 \mathrm{~Hz}, 2 \mathrm{H}), 1.97(\mathrm{~s}$, $3 \mathrm{H}), 1.82-1.51(\mathrm{~m}, 3 \mathrm{H}), 1.51-1.19(\mathrm{~m}, 7 \mathrm{H}), 0.88(\mathrm{~d}, J=6.93 \mathrm{~Hz}, 3 \mathrm{H})$. Minor diastereomer: $\delta 6.11(\mathrm{~s}, b r, 1 \mathrm{H}), 4.02(\mathrm{ddt}, J=9.76,8.24,5.30 \mathrm{~Hz}, 1 \mathrm{H}), 3.66(\mathrm{~s}, 3 \mathrm{H})$, $2.52(\mathrm{~d}, J=4.92 \mathrm{~Hz}, 2 \mathrm{H}), 1.96(\mathrm{~s}, 3 \mathrm{H}), 1.82-1.51(\mathrm{~m}, 3 \mathrm{H}), 1.51-1.19(\mathrm{~m}, 7 \mathrm{H}), 0.84$ $(\mathrm{d}, J=6.53 \mathrm{~Hz}, 3 \mathrm{H}) \cdot{ }^{13} \mathrm{C}$ NMR $(100 \mathrm{MHz}$, Chloroform- $d$ ) major diastereomer: $\delta$ 172.9, 169.8, 51.8, 48.3, 38.9, 36.1, 30.7, 30.7, 29.4, 25.6, 25.2, 23.5, 19.6. Minor diastereomer: $\delta 172.8,169.8,51.8,50.8,40.7,36.2,34.9,34.8,32.5,29.8,29.5,29.4$, 22.6. HRMS-ESI: Found $[\mathrm{M}+\mathrm{Na}]^{+}=264.1576 ; \mathrm{C}_{13} \mathrm{H}_{23} \mathrm{NO}_{3} \mathrm{Na}$ requires 264.1570.

Methyl (S)-3-acetamido-3-((1s,4R)-4-methoxycyclohexyl) propanoate<smiles>CO[C@H]1CC[C@H]([C@H](N)CC(C)=O)[C@H]1CC(C)=O</smiles>

Colorless oil. ${ }^{1} \mathrm{H}$ NMR (400 MHz, Chloroform- $d$ ) major diastereomer: $\delta 6.09(\mathrm{~d}, J=9.27 \mathrm{~Hz}, 1 \mathrm{H}), 4.09$ (ddt, $J=$ $10.90,9.06,5.00 \mathrm{~Hz}, 1 \mathrm{H}), 3.66(\mathrm{~s}, 3 \mathrm{H}), 3.39(\mathrm{tt}, J=10.48$,

$3.14 \mathrm{~Hz}, 1 \mathrm{H}), 3.25$ (s, 3H), $2.60-2.46(\mathrm{~m}, 2 \mathrm{H}), 2.13-2.02(\mathrm{~m}, 1 \mathrm{H}), 1.96(\mathrm{~s}, 3 \mathrm{H})$, $1.94-1.69(\mathrm{~m}, 2 \mathrm{H}), 1.60-1.25(\mathrm{~m}, 5 \mathrm{H}), 1.20-0.91(\mathrm{~m}, 1 \mathrm{H})$. Minor diastereomer: $\delta$ $6.04(\mathrm{~d}, J=9.14 \mathrm{~Hz}, 1 \mathrm{H}), 4.02$ (ddt, $J=11.26,9.67,5.00 \mathrm{~Hz}, 1 \mathrm{H}), 3.67$ (s, 3H), 3.31 (s, 3H), $3.04(\mathrm{tt}, J=10.48,3.14 \mathrm{~Hz}, 1 \mathrm{H}), 2.60-2.46(\mathrm{~m}, 2 \mathrm{H}), 2.13-2.02(\mathrm{~m}, 1 \mathrm{H})$, $1.96(\mathrm{~s}, 3 \mathrm{H}), 1.94-1.69(\mathrm{~m}, 2 \mathrm{H}), 1.60-1.25(\mathrm{~m}, 5 \mathrm{H}), 1.20-0.91(\mathrm{~m}, 1 \mathrm{H}) .{ }^{13} \mathrm{C} \mathrm{NMR}$ (100 MHz, Chloroform- $d$ ) major diastereomer: $\delta$ 172.8, 169.7, 74.5, 55.6, 51.8, 50.3, 40.1, 36.0, 29.2, 28.7, 23.7, 23.5, 23.2. Minor diastereomer: $\delta$ 172.7, 169.7, 79.2, 55.8, 51.9, 50.2, 40.3, 36.3, 31.4, 31.3, 27.9, 27.7, 23.5. HRMS-ESI: Found $[\mathrm{M}+\mathrm{Na}]^{+}=$ 280.1530; $\mathrm{C}_{13} \mathrm{H}_{23} \mathrm{NO}_{4} \mathrm{Na}$ requires 280.1519 . 


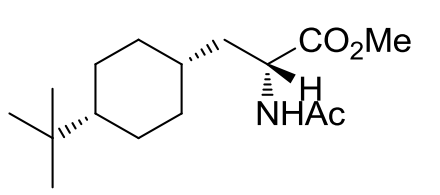

Colorless oil. ${ }^{1} \mathrm{H}$ NMR (400 MHz, Chloroform- $d$ ) major diastereomer: $\delta 5.88(\mathrm{~s}, b r, 1 \mathrm{H}), 4.59(\mathrm{td}, J=8.62,5.41 \mathrm{~Hz}$, $1 \mathrm{H}), 3.74$ (s, 3H), 2.01 (s, 3H), 1.92 (ddd, $J=13.82$, 8.32,

$5.44 \mathrm{~Hz}, 1 \mathrm{H}), 1.80-1.40(\mathrm{~m}, 8 \mathrm{H}), 1.15-0.89(\mathrm{~m}, 3 \mathrm{H}), 0.83(\mathrm{~s}, 9 \mathrm{H})$. Minor diastereomer: $\delta 5.88(\mathrm{~s}, b r, 1 \mathrm{H}), 4.65(\mathrm{td}, J=8.23,4.96 \mathrm{~Hz}, 1 \mathrm{H}), 3.72(\mathrm{~s}, 3 \mathrm{H}), 2.02(\mathrm{~s}$, $3 \mathrm{H}), 1.92(\mathrm{ddd}, J=13.82,8.32,5.44 \mathrm{~Hz}, 1 \mathrm{H}), 1.80-1.40(\mathrm{~m}, 8 \mathrm{H}), 1.15-0.89(\mathrm{~m}$, $3 \mathrm{H}), 0.82$ (s, 9H). ${ }^{13} \mathrm{C}$ NMR (100 MHz, Chloroform- $d$ ) major diastereomer: $\delta$ 173.9, 170.0, 52.4, 51.0, 48.5, 34.4, 32.7, 31.3, 30.0, 28.9, 27.6, 23.3, 21.9, 21.7. Minor diastereomer: $\delta 173.9,170.0,52.4,50.4,48.1,40.4,34.4,34.0,33.2,32.5,27.7,27.6$, 27.3, 27.2. HRMS-ESI: Found $[\mathrm{M}+\mathrm{Na}]^{+}=306.2048 ; \mathrm{C}_{16} \mathrm{H}_{29} \mathrm{NO}_{3} \mathrm{Na}$ requires 306.2040.

\section{Methyl $(R)-2$-acetamido-3-((1s,4S)-4-methylcyclohexyl) propanoate}

$\stackrel{\mathrm{I}_{\mathrm{H}}}{\mathrm{CO}_{2} \mathrm{Me}}$ Colorless oil. ${ }^{1} \mathrm{H}$ NMR $(400 \mathrm{MHz}$, Chloroform- $d$ ) major
diastereomer: $\delta 5.96(\mathrm{~s}, b r, 1 \mathrm{H}), 4.62(\mathrm{td}, J=8.44,5.22 \mathrm{~Hz}$ $1 \mathrm{H}), 3.72(\mathrm{~s}, 3 \mathrm{H}), 2.01(\mathrm{~s}, 3 \mathrm{H}), 1.87-1.75(\mathrm{~m}, 1 \mathrm{H}), 1.70-1.41(\mathrm{~m}, 7 \mathrm{H}), 1.41-1.28$ $(\mathrm{m}, 2 \mathrm{H}), 1.28-1.14(\mathrm{~m}, 2 \mathrm{H}), 0.88(\mathrm{~d}, J=6.86 \mathrm{~Hz}, 3 \mathrm{H})$. Minor diastereomer: $\delta 5.95(\mathrm{~s}$, $b r, 1 \mathrm{H}), 4.64(\mathrm{td}, J=8.44,5.22 \mathrm{~Hz}, 1 \mathrm{H}), 3.71(\mathrm{~s}, 3 \mathrm{H}), 2.01(\mathrm{~s}, 3 \mathrm{H}), 1.87-1.75(\mathrm{~m}$, $1 \mathrm{H}), 1.70-1.41(\mathrm{~m}, 7 \mathrm{H}), 1.41-1.28(\mathrm{~m}, 2 \mathrm{H}), 1.28-1.14(\mathrm{~m}, 2 \mathrm{H}), 0.85(\mathrm{~d}, J=6.57$ $\mathrm{Hz}, 3 \mathrm{H}) .{ }^{13} \mathrm{C}$ NMR (100 MHz, Chloroform- $d$ ) major diastereomer: $\delta$ 174.0, 170.0, 52.4, 50.6, 37.2, 31.5, 30.7, 30.5, 30.2, 29.3, 28.1, 23.3, 20.4. Minor diastereomer: $\delta$ 174.0, 169.9, 52.4, 50.3, 40.3, 35.2, 35.0, 34.1, 33.5, 32.7, 32.7, 32.6, 22.7. HRMSESI: Found $[\mathrm{M}+\mathrm{Na}]^{+}=264.1567 ; \mathrm{C}_{13} \mathrm{H}_{23} \mathrm{NO}_{3} \mathrm{Na}$ requires 264.1570 .

\section{Methyl $(R)$-2-acetamido -3- ((1s,4S)-4-(trifluoromethyl) cyclohexyl) propanoate}

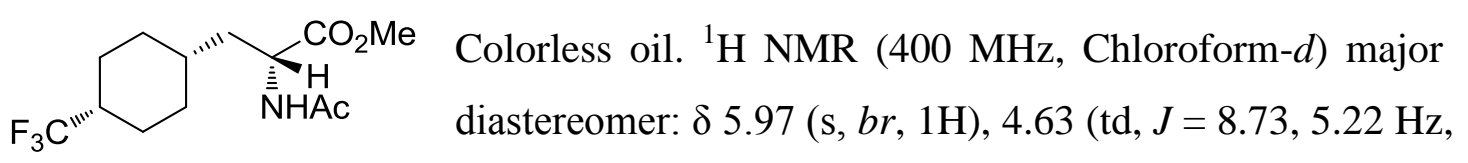
$1 \mathrm{H}), 3.74(\mathrm{~s}, 3 \mathrm{H}), 2.02(\mathrm{~s}, 3 \mathrm{H}), 2.00-1.81(\mathrm{~m}, 2 \mathrm{H}), 1.78-1.47(\mathrm{~m}, 8 \mathrm{H}), 1.37-1.20$ $(\mathrm{m}, 1 \mathrm{H}), 1.06-0.88(\mathrm{~m}, 1 \mathrm{H})$. Minor diastereomer: $5.95(\mathrm{~s}, b r, 1 \mathrm{H}), 4.68(\mathrm{dt}, J=5.50$, $3.20 \mathrm{~Hz}, 1 \mathrm{H}), 3.74(\mathrm{~s}, 3 \mathrm{H}), 2.02(\mathrm{~s}, 3 \mathrm{H}), 2.00-1.81(\mathrm{~m}, 2 \mathrm{H}), 1.78-1.47(\mathrm{~m}, 8 \mathrm{H})$, $1.37-1.20(\mathrm{~m}, 1 \mathrm{H}), 1.06-0.88(\mathrm{~m}, 1 \mathrm{H}) .{ }^{13} \mathrm{C} \mathrm{NMR}(100 \mathrm{MHz}$, Chloroform- $d$ ) major diastereomer: $\delta 173.6,170.1,128.06(\mathrm{q}, J=279.07 \mathrm{~Hz}), 52.6,50.6,40.57(\mathrm{q}, J=$ 
27.26 Hz), 35.8, 29.6, 29.0, 27.7, 23.3, 20.9, 20.6. Minor diastereomer: $\delta 173.6,170.0$, $128.06(\mathrm{q}, J=279.07 \mathrm{~Hz}), 52.6,50.1,42.0,41.8,40.57(\mathrm{q}, J=27.26 \mathrm{~Hz}), 33.6,31.8$, 31.0, 24.9, 24.8. ${ }^{19} \mathrm{~F}$ NMR (377 MHz, Chloroform- $d$ ) major diastereomer: $\delta-73.83$. Minor diastereomer: -72.03. HRMS-ESI: Found $[\mathrm{M}+\mathrm{Na}]^{+}=318.1281$; $\mathrm{C}_{13} \mathrm{H}_{20} \mathrm{~F}_{3} \mathrm{NO}_{3} \mathrm{Na}$ requires 318.1287.

\section{Methyl $(2 R, 3 S)$-2-acetamido-3-((1s,4R)-4-methylcyclohexyl) butanoate}<smiles>COC(=O)[C@H](N)[C@H](C)C1CCC(C)CC1</smiles>

Colorless oil. ${ }^{1} \mathrm{H}$ NMR (400 MHz, Chloroform- $d$ ) major diastereomer: $\delta 5.80(\mathrm{~d}, J=9.55 \mathrm{~Hz}, 1 \mathrm{H}), 4.92(\mathrm{dd}, J=9.53$, $2.75 \mathrm{~Hz}, 1 \mathrm{H}), 3.72(\mathrm{~s}, 3 \mathrm{H}), 2.04(\mathrm{~s}, 3 \mathrm{H}), 1.96-1.68(\mathrm{~m}, 5 \mathrm{H})$, $1.62-1.31(\mathrm{~m}, 4 \mathrm{H}), 1.19-1.07(\mathrm{~m}, 2 \mathrm{H}), 0.83(\mathrm{~d}, J=7.15 \mathrm{~Hz}, 3 \mathrm{H}), 0.78(\mathrm{~d}, J=6.86$ $\mathrm{Hz}, 3 \mathrm{H})$. Minor diastereomer: $\delta 5.96(\mathrm{~d}, J=9.10 \mathrm{~Hz}, 1 \mathrm{H}), 4.57(\mathrm{dd}, J=9.37 \mathrm{~Hz}, 1 \mathrm{H})$, $3.63(\mathrm{~s}, 3 \mathrm{H}), 2.98(\mathrm{dq}, J=9.47,7.02 \mathrm{~Hz}, 1 \mathrm{H}), 2.01(\mathrm{~s}, 3 \mathrm{H}), 1.96-1.68(\mathrm{~m}, 4 \mathrm{H}), 1.62$ - $1.31(\mathrm{~m}, 4 \mathrm{H}), 1.19-0,87(\mathrm{~m}, 8 \mathrm{H}) \cdot{ }^{13} \mathrm{C}$ NMR (100 MHz, Chloroform- $d$ ) major diastereomer: $\delta$ 173.9, 170.4, 53.3, 52.4, 41.9, 37.7, 34.0, 28.7, 26.9, 24.1, 23.4, 20.2, 11.9, 11.8. Minor diastereomer: $\delta 173.3,169.9,55.8,52.0,39.3,32.7,28.7,24.3,23.4$, 23.3, 23.1, 20.2, 19.3, 15.2. HRMS-ESI: Found $[\mathrm{M}+\mathrm{Na}]^{+}=278.1727 ; \mathrm{C}_{14} \mathrm{H}_{25} \mathrm{NO}_{3} \mathrm{Na}$ requires 278.1727 .

\section{$N-((1 S, 3 a R, 7 a R)-o c t a h y d r o-1 H$-inden-1-yl)acetamide}<smiles>CNC1CC[C@@H]2CCCC[C@@H]12</smiles>

White solid. ${ }^{1} \mathrm{H}$ NMR (400 MHz, Chloroform- $d$ ) major diastereomer: $\delta 5.54(\mathrm{~s}, b r, 1 \mathrm{H}), 4.28-4.11(\mathrm{~m}, 1 \mathrm{H}), 2.30-1.99(\mathrm{~m}, 2 \mathrm{H}), 1.96(\mathrm{~s}$, $3 \mathrm{H}), 1.79-1.60(\mathrm{~m}, 2 \mathrm{H}), 1.57-1.25(\mathrm{~m}, 8 \mathrm{H}), 1.20-0.87(\mathrm{~m}, 2 \mathrm{H})$. Minor diastereomer: $\delta 5.58(\mathrm{~s}, b r, 1 \mathrm{H}), 4.28-4.11(\mathrm{~m}, 1 \mathrm{H}), 2.30-1.99(\mathrm{~m}, 2 \mathrm{H}), 1.96$ (s, 3H), $1.79-1.60(\mathrm{~m}, 2 \mathrm{H}), 1.57-1.25(\mathrm{~m}, 8 \mathrm{H}), 1.20-0.87(\mathrm{~m}, 2 \mathrm{H}) .{ }^{13} \mathrm{C}$ NMR $(100$ MHz, Chloroform- $d$ ) major diastereomer: $\delta$ 170.0, 54.0, 41.1, 37.0, 28.9, 26.8, 25.0, 24.0, 23.4, 21.9, 20.9. Minor diastereomer: $\delta$ 170.1, 52.5, 46.2, 37.8, 30.9, 28.5, 28.4, 25.1, 24.1, 23.5, 22.4. HRMS-ESI: Found $[\mathrm{M}+\mathrm{Na}]^{+}=204.1356 ; \mathrm{C}_{11} \mathrm{H}_{19} \mathrm{NONa}$ requires 204.1359 . 


\section{$N$-((1S,3aR,7aR)-octahydro-1H-inden-1-yl)isobutyramide}

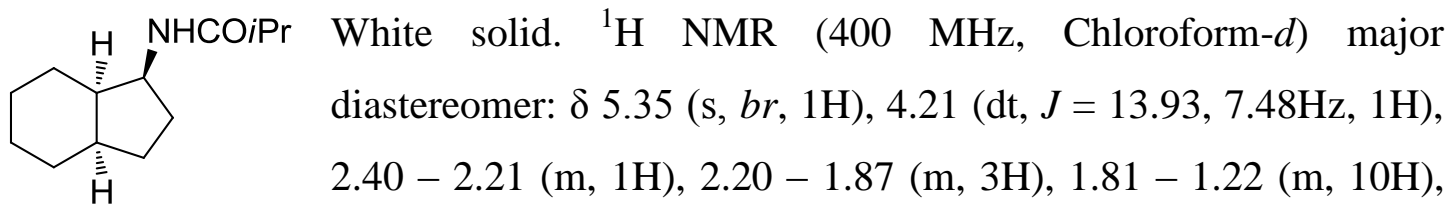

$1.14(\mathrm{~d}, J=6.86 \mathrm{~Hz}, 6 \mathrm{H}), 0.93(\mathrm{qd}, J=12.63,3.37 \mathrm{~Hz}, 1 \mathrm{H})$. Minor diastereomer: $\delta$ $5.35(\mathrm{~s}, b r, 1 \mathrm{H}), 4.21(\mathrm{dt}, J=13.93,7.48 \mathrm{~Hz}, 1 \mathrm{H}), 2.40-2.21(\mathrm{~m}, 1 \mathrm{H}), 2.20-1.87(\mathrm{~m}$, $3 \mathrm{H}), 1.81-1.22(\mathrm{~m}, 10 \mathrm{H}), 1.14(\mathrm{~d}, J=6.86 \mathrm{~Hz}, 6 \mathrm{H}), 0.93(\mathrm{qd}, J=12.63,3.37 \mathrm{~Hz}$, 1H). ${ }^{13} \mathrm{C}$ NMR (100 MHz, Chloroform- $d$ ) major diastereomer: $\delta$ 176.6, 53.5, 40.9, 36.9, 29.0, 26.8, 24.9, 24.0, 21.7, 20.8, 19.9, 19.6. Minor diastereomer: $\delta$ 176.8, 52.0, 46.3, 37.8, 35.7, 30.9, 28.4, 28.3, 25.1, 24.0, 22.4, 19.8. HRMS-ESI: Found [M+Na] ${ }^{+}$ $=232.1672 ; \mathrm{C}_{13} \mathrm{H}_{23} \mathrm{NONa}$ requires 232.1644 .

\section{$N$-((1S,3a $R, 7 \mathrm{a} R)$-octahydro-1H-inden-1-yl)pivalamide}

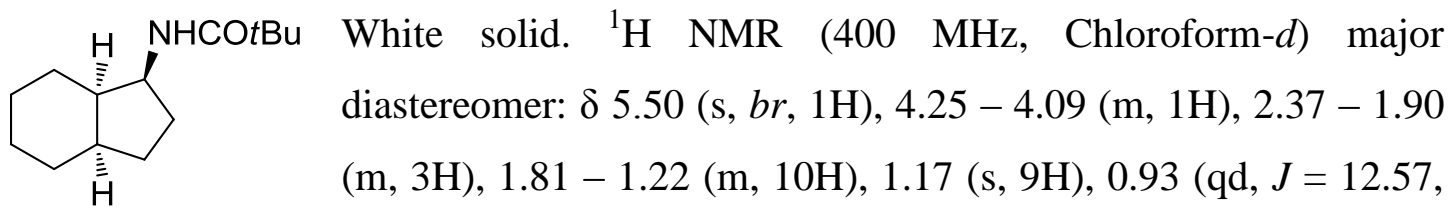
$3.47 \mathrm{~Hz}, 1 \mathrm{H})$. Minor diastereomer: $\delta 5.44(\mathrm{~s}, b r, 1 \mathrm{H}), 4.25-4.09(\mathrm{~m}, 1 \mathrm{H}), 2.37-1.90$ $(\mathrm{m}, 3 \mathrm{H}), 1.81-1.22(\mathrm{~m}, 10 \mathrm{H}), 1.17(\mathrm{~s}, 9 \mathrm{H}), 0.93(\mathrm{qd}, J=12.57,3.47 \mathrm{~Hz}, 1 \mathrm{H}) .{ }^{13} \mathrm{C}$ NMR (100 MHz, Chloroform-d) major diastereomer: $\delta 178.2$, 53.7, 41.0, 38.7, 37.1, 29.2, 27.8, 26.9, 25.0, 24.1, 21.8, 21.0. Minor diastereomer: $\delta 178.3,52.2,46.5,38.6$, 37.9, 31.0, 28.9, 28.5, 28.4, 27.8, 25.3, 22.6. HRMS-ESI: Found $[\mathrm{M}+\mathrm{Na}]^{+}=$ 246.1826; $\mathrm{C}_{14} \mathrm{H}_{125} \mathrm{NONa}$ requires 246.1828.

\section{2,2,2-trifluoro- $N-((1 S, 3 a R, 7 \mathrm{a} R)$-octahydro-1H-inden-1-yl)acetamide}

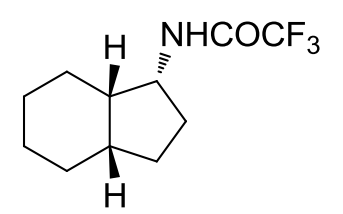

White solid. ${ }^{1} \mathrm{H}$ NMR (400 MHz, Chloroform- $d$ ) $\delta 6.14$ (s, br, $1 \mathrm{H}), 4.26(\mathrm{qd}, J=8.99,6.05 \mathrm{~Hz}, 1 \mathrm{H}), 2.38-2.00(\mathrm{~m}, 3 \mathrm{H}), 1.86-$ $1.26(\mathrm{~m}, 9 \mathrm{H}), 1.17$ (qt, $J=12.73,3.25 \mathrm{~Hz}, 1 \mathrm{H}), 0.98$ (qd, $J=$ 12.51, 3.49 Hz, 1H). ${ }^{13} \mathrm{C}$ NMR (100 MHz, Chloroform- $d$ ) $\delta 157.1$ (q, $J=36.44 \mathrm{~Hz}$ ), $116.0(\mathrm{q}, J=289.79 \mathrm{~Hz}), 54.4,40.9,37.0,28.8,26.7,24.8,24.0,21.7,20.8 .{ }^{19} \mathrm{~F}$ NMR $(377 \mathrm{MHz}$, Chloroform- $d) \delta-75.93 .[\alpha]_{\mathrm{D}}^{29}=76.5\left(\mathrm{c}=0.2, \mathrm{CHCl}_{3}\right)$. HRMS-ESI: Found $[\mathrm{M}+\mathrm{Na}]^{+}=258.1086 ; \mathrm{C}_{11} \mathrm{H}_{16} \mathrm{NOF}_{3} \mathrm{Na}$ requires 258.1076 . 


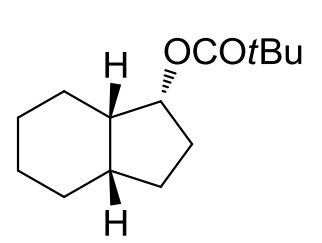

Colorless oil. ${ }^{1} \mathrm{H}$ NMR (400 MHz, Chloroform- $d$ ) $\delta 5.00(\mathrm{dt}, J=$ 8.67, $6.19 \mathrm{~Hz}, 1 \mathrm{H}), 2.15-1.97(\mathrm{~m}, 3 \mathrm{H}), 1.74-1.58(\mathrm{~m}, 3 \mathrm{H})$, $1.59-1.35(\mathrm{~m}, 7 \mathrm{H}), 1.22-1.12(\mathrm{~m}, 10 \mathrm{H}) .{ }^{13} \mathrm{C} \mathrm{NMR}(100 \mathrm{MHz}$,

Chloroform- $d$ ) $\delta$ 178.6, 78.2, 41.3, 38.9, 36.4, 28.9, 27.4, 27.3, 24.9, 24.7, 21.9, 21.6. $[\alpha]_{\mathrm{D}}{ }^{29}=17.5\left(\mathrm{c}=0.2, \mathrm{CHCl}_{3}\right)$. HRMS-ESI: Found $[\mathrm{M}+\mathrm{Na}]^{+}=$ 247.1676; $\mathrm{C}_{14} \mathrm{H}_{24} \mathrm{NONa}$ requires 247.1669.

\section{$N-((1 R, 2 R, 3 a S, 7 \mathrm{a} S)-2-m e t h y l o c t a h y d r o-1 H$-inden-1-yl) pivalamide}

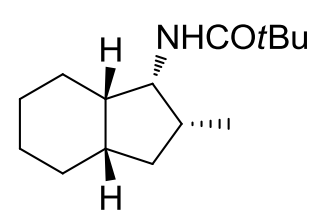

White solid. ${ }^{1} \mathrm{H}$ NMR (400 MHz, Chloroform- $d$ ) $\delta 5.73$ (s, br, $1 \mathrm{H}), 4.33(\mathrm{td}, J=10.15,6.20 \mathrm{~Hz}, 1 \mathrm{H}), 2.34$ (dddd, $J=17.40,8.72$, $7.27,2.07 \mathrm{~Hz}, 1 \mathrm{H}), 2.09-1.96(\mathrm{~m}, 2 \mathrm{H}), 1.79(\mathrm{dt}, J=12.60,7.79$ $\mathrm{Hz}, 1 \mathrm{H}), 1.73-1.64(\mathrm{~m}, 1 \mathrm{H}), 1.63-1.29(\mathrm{~m}, 6 \mathrm{H}), 1.20(\mathrm{~s}, 9 \mathrm{H}), 1.19-0.98(\mathrm{~m}, 2 \mathrm{H})$, $0.91(\mathrm{~d}, J=7.28 \mathrm{~Hz}, 3 \mathrm{H}) .{ }^{13} \mathrm{C}$ NMR (100 MHz, Chloroform- $d$ ) $\delta$ 177.9, 54.1, 41.9, $39.1,36.2,35.8,33.5,27.9,27.0,25.4,23.3,21.1,18.0 .[\alpha]_{\mathrm{D}}{ }^{29}=-4.0\left(\mathrm{c}=0.2, \mathrm{CHCl}_{3}\right)$. HRMS-ESI: Found $[\mathrm{M}+\mathrm{Na}]^{+}=260.1986 ; \mathrm{C}_{15} \mathrm{H}_{27} \mathrm{NONa}$ requires 260.1985 .

\section{$N$-((1R,2R,3aS,7aS)-2-ethyloctahydro-1H-inden-1-yl)pivalamide}

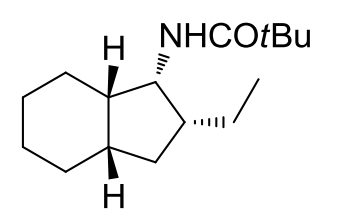

White solid. ${ }^{1} \mathrm{H}$ NMR (400 MHz, Chloroform- $d$ ) $\delta 5.69$ (s, br, $1 \mathrm{H}), 4.40(\mathrm{td}, J=9.69,5.98 \mathrm{~Hz}, 1 \mathrm{H}), 2.16-1.92(\mathrm{~m}, 3 \mathrm{H}), 1.83-$ $1.72(\mathrm{~m}, 1 \mathrm{H}), 1.62(\mathrm{ddt}, J=24.31,11.34,4.27 \mathrm{~Hz}, 2 \mathrm{H}), 1.55-$ $1.34(\mathrm{~m}, 5 \mathrm{H}), 1.35-1.22(\mathrm{~m}, 1 \mathrm{H}), 1.21(\mathrm{~s}, 9 \mathrm{H}), 1.14-0.95(\mathrm{~m}, 3 \mathrm{H}), 0.87(\mathrm{t}, J=7.26$ $\mathrm{Hz}, 3 \mathrm{H}) .{ }^{13} \mathrm{C}$ NMR (100 MHz, Chloroform- $d$ ) $\delta$ 177.8, 53.9, 41.8, 41.3, 39.1, 36.0, $33.9,27.9,27.4,25.9,25.2,23.2,21.2,13.0 .[\alpha]_{\mathrm{D}}{ }^{29}=-10.5\left(\mathrm{c}=0.2, \mathrm{CHCl}_{3}\right)$. HRMSESI: Found $[\mathrm{M}+\mathrm{Na}]^{+}=274.2142 ; \mathrm{C}_{16} \mathrm{H}_{29} \mathrm{NONa}$ requires 274.2141 .

\section{2,2,2-trifluoro- $N$-((1R,3a $R, 5 R, 7 \mathrm{a} S)-5$-methyloctahydro- $1 H$-inden-1-yl)acetamide}

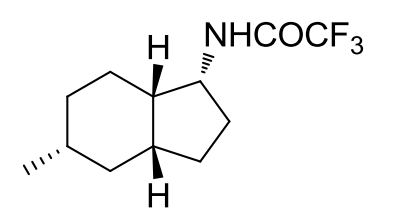

Colorless oil. ${ }^{1} \mathrm{H}$ NMR (400 MHz, Chloroform- $d$ ) $\delta 6.10$ (s, $b r$, $1 \mathrm{H}), 4.43(\mathrm{td}, J=8.73,6.35 \mathrm{~Hz}, 1 \mathrm{H}), 2.23-2.13(\mathrm{~m}, 2 \mathrm{H})$, 2.06 (dddd, $J=14.42,12.53,6.44,2.45 \mathrm{~Hz}, 1 \mathrm{H}$ ), $1.84-1.34$ $(\mathrm{m}, 8 \mathrm{H}), 0.91(\mathrm{~d}, J=6.63 \mathrm{~Hz}, 3 \mathrm{H}), 0.85-0.72(\mathrm{~m}, 1 \mathrm{H}), 0.65(\mathrm{dt}, J=13.51,11.52 \mathrm{~Hz}$, 1H). ${ }^{13} \mathrm{C}$ NMR (100 MHz, Chloroform-d) $\delta 156.9$ (q, $\left.J=36.4 \mathrm{~Hz}\right), 116.1$ (q, $J=$ $288.1 \mathrm{~Hz}), 53.8,39.9,38.5,38.4,32.3,31.8,30.4,30.1,22.9,22.4 .{ }^{19} \mathrm{~F}$ NMR (377 
MHz, Chloroform- $d$ ) $\delta$-75.97. $[\alpha]_{\mathrm{D}}^{29}=60.5\left(\mathrm{c}=0.2, \mathrm{CHCl}_{3}\right)$. HRMS-ESI: Found $[\mathrm{M}+\mathrm{Na}]^{+}=272.1232 ; \mathrm{C}_{12} \mathrm{H}_{18} \mathrm{NOF}_{3} \mathrm{Na}$ requires 272.1233.

\section{2,2,2-trifluoro- $N$-((1R,3a $R, 4 R, 7 \mathrm{a} S)-4$-methyloctahydro-1 $H$-inden-1-yl) acetamide}

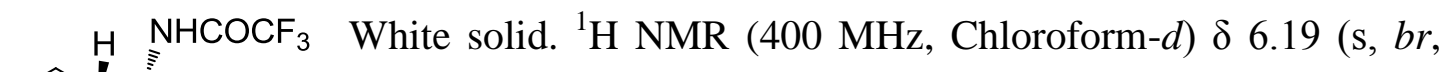
$1 \mathrm{H}), 4.25(\mathrm{qd}, J=8.90,5.91 \mathrm{~Hz}, 1 \mathrm{H}), 2.25-1.99(\mathrm{~m}, 3 \mathrm{H}), 1.74$ $(\mathrm{dqd}, J=13.75,6.83,3.78 \mathrm{~Hz}, 2 \mathrm{H}), 1.66-1.31(\mathrm{~m}, 5 \mathrm{H}), 1.21$ (qt, $J=12.84,3.18 \mathrm{~Hz}, 1 \mathrm{H}), 1.07(\mathrm{qd}, J=12.83,3.22 \mathrm{~Hz}, 1 \mathrm{H}), 0.95-0.87(\mathrm{~m}, 1 \mathrm{H}), 0.85$ $(\mathrm{d}, J=6.83 \mathrm{~Hz}, 3 \mathrm{H}) .{ }^{13} \mathrm{C}$ NMR $(100 \mathrm{MHz}$, Chloroform- $d) \delta 156.8(\mathrm{q}, J=35.43 \mathrm{~Hz})$, $115.9(\mathrm{~d}, J=289.79 \mathrm{~Hz}), 53.8,43.1,41.8,31.9,28.6,27.8,24.9,21.1,19.9,19.3 .{ }^{19} \mathrm{~F}$ NMR $(377 \mathrm{MHz}$, Chloroform- $d) \delta-75.87 .[\alpha]_{\mathrm{D}}{ }^{29}=72.5\left(\mathrm{c}=0.2, \mathrm{CHCl}_{3}\right)$. HRMS-ESI: Found $[\mathrm{M}+\mathrm{Na}]^{+}=272.1227 ; \mathrm{C}_{12} \mathrm{H}_{18} \mathrm{NOF}_{3} \mathrm{Na}$ requires 272.1233 .

2,2,2-trifluoro- $N-((1 R, 3 \mathrm{a} R, 6 S 7 \mathrm{a} S) 6$-methoxyoctahydro-1 $H$-inden-1-yl) acetamide $b r, 1 \mathrm{H}), 4.20(\mathrm{td}, J=7.61 \mathrm{~Hz}, 1 \mathrm{H}), 3.41-3.25(\mathrm{~m}, 4 \mathrm{H})$,
$2.34-2.23(\mathrm{~m}, 1 \mathrm{H}), 2.26-2.12(\mathrm{~m}, 1 \mathrm{H}), 2.12-1.99(\mathrm{~m}$, 1H), 1.82 - $1.34(\mathrm{~m}, 9 \mathrm{H}) .{ }^{13} \mathrm{C}$ NMR (100 MHz, Chloroform-d) $\delta 157.2$ (q, $J=36.5$ Hz), $116.2(\mathrm{q}, J=288.0 \mathrm{~Hz}), 75.7,55.8,53.0,39.2,37.5,30.8,28.4,27.8,26.2,23.6$. ${ }^{19} \mathrm{~F}$ NMR $(377 \mathrm{MHz}$, Chloroform- $d) \delta$-75.91. $[\alpha]_{\mathrm{D}}{ }^{29}=20.5\left(\mathrm{c}=0.2, \mathrm{CHCl}_{3}\right)$. HRMSESI: Found $[\mathrm{M}+\mathrm{Na}]^{+}=288.1183 ; \mathrm{C}_{12} \mathrm{H}_{18} \mathrm{NO}_{2} \mathrm{~F}_{3} \mathrm{Na}$ requires 288.1182.

\section{$N$-((1R,3aR,4R,7aS)4-ethyloctahydro-1H-inden-1-yl)-2,2,2-trifluoroacetamide}

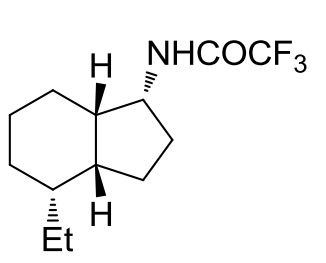

Colorless oil. ${ }^{1} \mathrm{H}$ NMR (400 MHz, Chloroform- $d$ ) $\delta 6.16$ (s, br, $1 \mathrm{H}), 4.25$ (dt, $J=8.96,4.35 \mathrm{~Hz}, 1 \mathrm{H}), 2.23-2.04$ (m, 3H), 1.79 $(\operatorname{tp}, J=13.20,4.06,3.38 \mathrm{~Hz}, 1 \mathrm{H}), 1.64-1.32(\mathrm{~m}, 6 \mathrm{H}), 1.30-$ $1.12(\mathrm{~m}, 3 \mathrm{H}), 1.08-0.92(\mathrm{~m}, 2 \mathrm{H}), 0.88(\mathrm{t}, J=7.33 \mathrm{~Hz}, 3 \mathrm{H}) .{ }^{13} \mathrm{C}$ NMR (100 MHz, Chloroform- $d$ ) $\delta 156.9$ (q, $J=36.6 \mathrm{~Hz}), 116.0$ (q, $J=288.3 \mathrm{~Hz}$ ), 53.9, 41.9, 41.1, 39.1, 28.0, 27.3, 26.9, 25.0, 21.7, 19.4, 11.8. ${ }^{19} \mathrm{~F}$ NMR (377 MHz, Chloroform- $d$ ) $\delta-75.86 .[\alpha]_{\mathrm{D}}{ }^{29}=68.0\left(\mathrm{c}=0.2, \mathrm{CHCl}_{3}\right)$. HRMS-ESI: Found $[\mathrm{M}+\mathrm{Na}]^{+}$ $=286.1394 ; \mathrm{C}_{13} \mathrm{H}_{20} \mathrm{NOF}_{3} \mathrm{Na}$ requires 286.1389. 


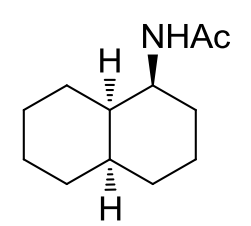

White solid. ${ }^{1} \mathrm{H}$ NMR (400 MHz, Chloroform- $d$ ) major diastereomer: $\delta$ $6.01(\mathrm{~s}, b r, 1 \mathrm{H}), 3.87(\mathrm{td}, J=8.07,4.30 \mathrm{~Hz}, 1 \mathrm{H}), 2.01(\mathrm{~s}, 3 \mathrm{H}), 1.98-$ $1.87(\mathrm{~m}, 1 \mathrm{H}), 1.84-1.64(\mathrm{~m}, 3 \mathrm{H}), 1.63-1.12(\mathrm{~m}, 12 \mathrm{H})$. Minor diastereomer: $\delta 6.19(\mathrm{~s}, b r, 1 \mathrm{H}), 4.06(\mathrm{td}, J=8.07,4.30 \mathrm{~Hz}, 1 \mathrm{H}), 2.05$ $(\mathrm{s}, 3 \mathrm{H}), 1.98-1.87(\mathrm{~m}, 1 \mathrm{H}), 1.84-1.64(\mathrm{~m}, 3 \mathrm{H}), 1.63-1.12(\mathrm{~m}, 12 \mathrm{H}) .{ }^{13} \mathrm{C} \mathrm{NMR}$ (100 MHz, Chloroform-d) major diastereomer: $\delta$ 170.1, 52.4, 39.7, 36.0, 31.9, 26.8, 26.4, 25.2, 24.5, 23.2, 21.3, 19.9. Minor diastereomer: $\delta$ 170.7, 41.5, 39.7, 36.0, 31.9, 26.8, 26.4, 25.2, 22.9, 21.3, 20.9, 19.9. HRMS-ESI: Found $[\mathrm{M}+\mathrm{Na}]^{+}=218.1515$; $\mathrm{C}_{12} \mathrm{H}_{21} \mathrm{NONa}$ requires 218.1514 .

\section{$N$-((1S,4aR,8aR)-decahydronaphthalen-1-yl)pivalamide}

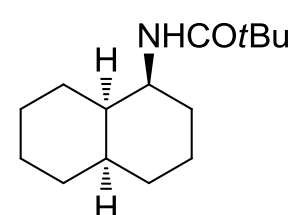

White solid. ${ }^{1} \mathrm{H}$ NMR (400 MHz, Chloroform- $d$ ) $\delta 5.50(\mathrm{~s}, b r, 1 \mathrm{H})$, 3.87 (ddt, $J=12.53,8.53,4.40 \mathrm{~Hz}, 1 \mathrm{H}), 2.00-1.89(\mathrm{~m}, 1 \mathrm{H}), 1.82$ - 1.69 (m, 3H), $1.66-1.20(\mathrm{~m}, 12 \mathrm{H}), 1.18$ (s, 9H). ${ }^{13} \mathrm{C}$ NMR $(100$ MHz, Chloroform- $d$ ) $\delta$ 177.5, 51.4, 36.0, 31.9, 27.8, 27.8, 27.1, 26.5, 25.3, 24.6, 21.4, 20.0. $[\alpha]_{\mathrm{D}}{ }^{29}=-13.0\left(\mathrm{c}=0.2, \mathrm{CHCl}_{3}\right)$. HRMS-ESI: Found $[\mathrm{M}+\mathrm{Na}]^{+}=260.1980$; $\mathrm{C}_{15} \mathrm{H}_{27} \mathrm{NONa}$ requires 260.1985 .

\section{$(1 R, 4 a S, 8 a S)$-decahydronaphthalen-1-yl pivalate}

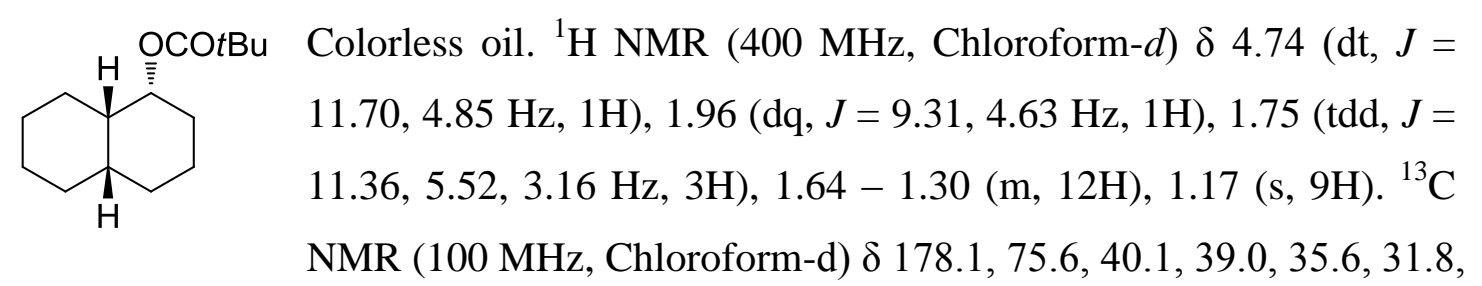
27.4, 26.3, 25.9, 24.6, 24.2, 21.5, 20.1. $[\alpha]_{\mathrm{D}}{ }^{29}=44.0\left(\mathrm{c}=0.2, \mathrm{CHCl}_{3}\right)$. HRMS-ESI: Found $[\mathrm{M}+\mathrm{Na}]^{+}=261.1835 ; \mathrm{C}_{15} \mathrm{H}_{26} \mathrm{NO}_{2} \mathrm{Na}$ requires 261.1825 .

\section{2,2,2-trifluoro- $N$-((4R,4a $R, 8 \mathrm{a} R)$-octahydro-2H-chromen-4-yl)acetamide}

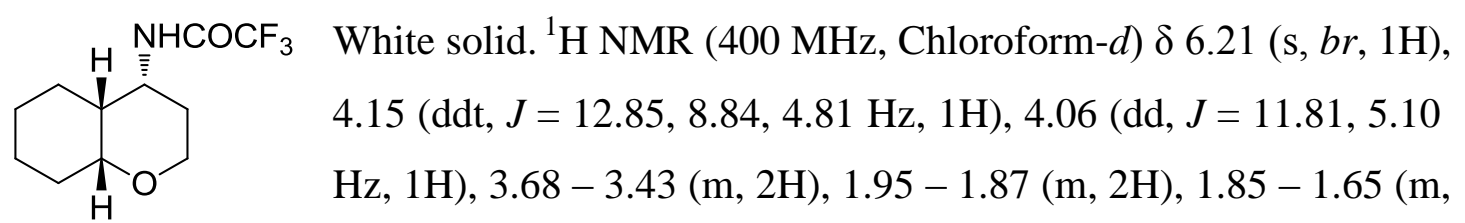
2H), $1.64-1.39(\mathrm{~m}, 5 \mathrm{H}), 1.36-1.16(\mathrm{~m}, 2 \mathrm{H}) .{ }^{13} \mathrm{C}$ NMR $(100 \mathrm{MHz}$, Chloroform- $d) \delta$ $156.5(\mathrm{q}, J=36.67 \mathrm{~Hz}), 115.9(\mathrm{q}, J=288.21 \mathrm{~Hz}), 75.0,66.8,50.5,38.7,31.6,26.9$, 
25.2, 20.6, 19.8. ${ }^{19} \mathrm{~F}$ NMR (377 MHz, Chloroform- $d$ ) $\delta-75.93 .[\alpha]_{\mathrm{D}}{ }^{29}=64.5(\mathrm{c}=0.2$, $\left.\mathrm{CHCl}_{3}\right)$. HRMS-ESI: Found $[\mathrm{M}+\mathrm{Na}]^{+}=274.1033 ; \mathrm{C}_{11} \mathrm{H}_{16} \mathrm{~F}_{3} \mathrm{NO}_{2} \mathrm{Na}$ requires 274.1025 .

\section{$(1 R, 4 \mathrm{a} R, 5 R, 8 \mathrm{a} S)-5-$ methyldecahydronaphthalen-1-yl pivalate}

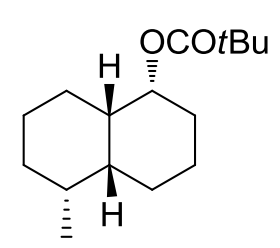

Colorless oil. ${ }^{1} \mathrm{H}$ NMR (400 MHz, Chloroform- $d$ ) $\delta 4.75$ (dt, $J=$ $11.90,4.85 \mathrm{~Hz}, 1 \mathrm{H}), 2.00-1.90(\mathrm{~m}, 1 \mathrm{H}), 1.85-1.72(\mathrm{~m}, 2 \mathrm{H}), 1.66$ $-1.21(\mathrm{~m}, 12 \mathrm{H}), 1.18(\mathrm{~s}, 9 \mathrm{H}), 0.86(\mathrm{~d}, J=6.75 \mathrm{~Hz}, 3 \mathrm{H}) .{ }^{13} \mathrm{C} \mathrm{NMR}$ (100 MHz, Chloroform- $d$ ) $\delta 178.1,75.6,41.6,41.5,39.0,35.9,29.6$, 27.4, 26.3, 26.2, 24.1, 20.0, 19.4, 18.5. $[\alpha]_{\mathrm{D}}{ }^{29}=42.0\left(\mathrm{c}=0.2, \mathrm{CHCl}_{3}\right)$. HRMS-ESI: Found $[\mathrm{M}+\mathrm{Na}]^{+}=275.1983 ; \mathrm{C}_{16} \mathrm{H}_{28} \mathrm{NO}_{2} \mathrm{Na}$ requires 275.1982 .

\section{$(1 R, 4 \mathrm{a} S, 5 R, 8 \mathrm{a} S)-5-$-methoxydecahydronaphthalen-1-yl pivalate}

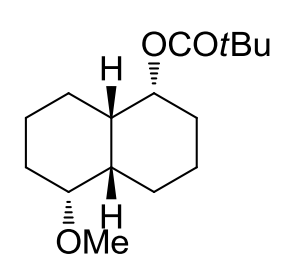

Colorless oil. ${ }^{1} \mathrm{H}$ NMR (400 MHz, Chloroform- $d$ ) $\delta 4.76$ (dt, $J=$ $12.02,4.89 \mathrm{~Hz}, 1 \mathrm{H}), 3.31(\mathrm{~s}, 3 \mathrm{H}), 3.16(\mathrm{dt}, J=11.78,4.61 \mathrm{~Hz}, 1 \mathrm{H})$, $2.10(\mathrm{dq}, J=12.39,4.17 \mathrm{~Hz}, 1 \mathrm{H}), 1.94(\mathrm{td}, J=9.26,4.58 \mathrm{~Hz}, 1 \mathrm{H})$, $1.87-1.62(\mathrm{~m}, 4 \mathrm{H}), 1.57-1.20(\mathrm{~m}, 8 \mathrm{H}), 1.18(\mathrm{~s}, 9 \mathrm{H}) .{ }^{13} \mathrm{C} \mathrm{NMR}$ (100 MHz, Chloroform- $d$ ) $\delta 178.1,81.7,75.1,55.8,39.6,39.0,38.8,27.4,26.9,26.5$, 23.9, 23.8, 19.2, 18.2. $[\alpha]_{\mathrm{D}}^{29}=89.5\left(\mathrm{c}=0.2, \mathrm{CHCl}_{3}\right)$. HRMS-ESI: Found $[\mathrm{M}+\mathrm{Na}]^{+}=$ 291.1937; $\mathrm{C}_{16} \mathrm{H}_{28} \mathrm{NO}_{3} \mathrm{Na}$ requires 291.1931.

\section{$(1 R, 4 \mathrm{a} R, 7 \mathrm{~S}, 8 \mathrm{a} S)-7-$ methyldecahydronaphthalen-1-yl pivalate}

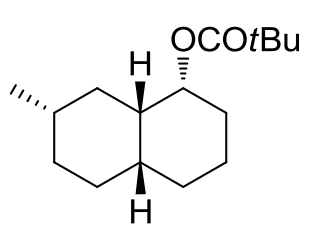

Colorless oil. ${ }^{1} \mathrm{H}$ NMR (400 MHz, Chloroform- $d$ ) $\delta 4.76$ (dt, $J=$ $11.79,4.86 \mathrm{~Hz}, 1 \mathrm{H}), 2.01(\mathrm{dq}, J=12.78,4.13 \mathrm{~Hz}, 1 \mathrm{H}), 1.82-$ $1.66(\mathrm{~m}, 2 \mathrm{H}), 1.61-1.24(\mathrm{~m}, 10 \mathrm{H}), 1.18(\mathrm{~s}, 9 \mathrm{H}), 1.16-1.01(\mathrm{~m}$, 2H), $0.91(\mathrm{~d}, J=6.42 \mathrm{~Hz}, 3 \mathrm{H}) .{ }^{13} \mathrm{C}$ NMR $(100 \mathrm{MHz}$, Chloroform-

d) $\delta 178.1,75.5,40.1,39.0,35.1,32.7,31.7,30.2,28.7,27.4,25.8,24.5,24.3,23.2$. $[\alpha]_{\mathrm{D}}^{29}=46.0\left(\mathrm{c}=0.2, \mathrm{CHCl}_{3}\right)$. HRMS-ESI: Found $[\mathrm{M}+\mathrm{Na}]^{+}=275.1983$; $\mathrm{C}_{16} \mathrm{H}_{28} \mathrm{NO}_{2} \mathrm{Na}$ requires 275.1982 . 


\section{Assignment of the absolute configurations of hydrogenated products.}

The absolute configuration of hydrogenated product $\mathbf{3 d}$ was determined as shown in Fig. S1. Firstly, compound 1d was subjected to hydrogenation to give compound 5d, which was confirmed as $R$ configuration. ${ }^{13}$ Compound $\mathbf{1 d}$ was then hydrogenated by same Rh precursor and ligand to yield product 3d. Finally, deprotection of compound 3d gave compound $\mathbf{7}$, which was reported in literature. ${ }^{14}$

a)

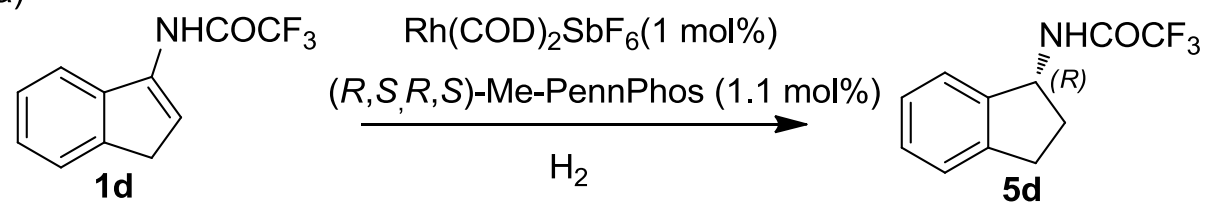

b)<smiles>NC1=CCc2ccccc21</smiles>

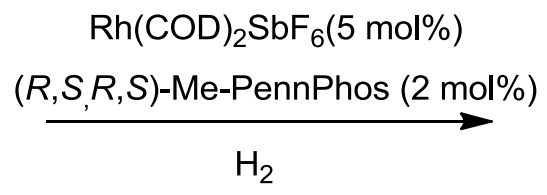<smiles>C[C@@]12CCCC[C@@H]1[C@@H](NC(=O)C(F)(F)F)CC2</smiles><smiles>N[C@H]1CC[C@@H]2CCCC[C@H]12</smiles>

cis-cis-7 cis-cis

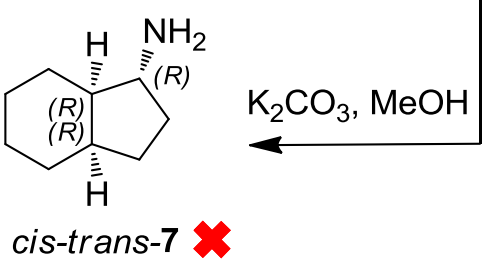

known compounds

Figure S1. Assignment of the absolute configurations of hydrogenated products $3 \mathbf{d}$.

The absolute configuration of another hydrogenated product $\mathbf{3 n}$ was determined as shown in Figure S2. Firstly, 1-tetralone was fully hydrogenated to give the mixtures of 1-decalols. The diastereomer 9 was then separated from the mixture, and the relative configuration was confirmed by NMR spectra according to literature. ${ }^{15}$ Finally, the absolute configuration of $\mathbf{3 n}$ was confirmed by comparison of the NMR data with ester derivative of 9. 

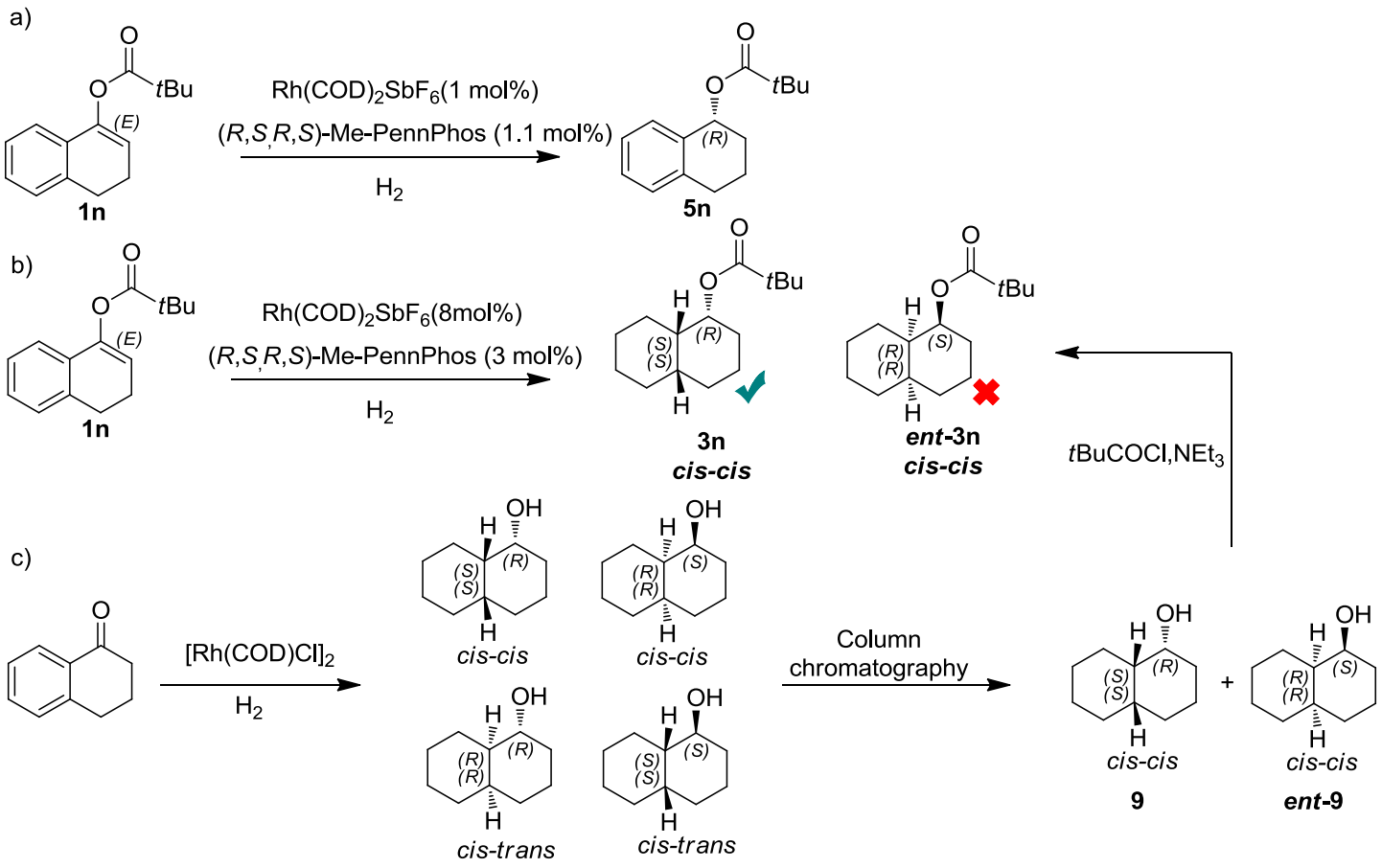

Figure S2. Assignment of the absolute configurations of hydrogenated product $\mathbf{3 n}$.

\section{Scale-up asymmetric hydrogenations and applications}
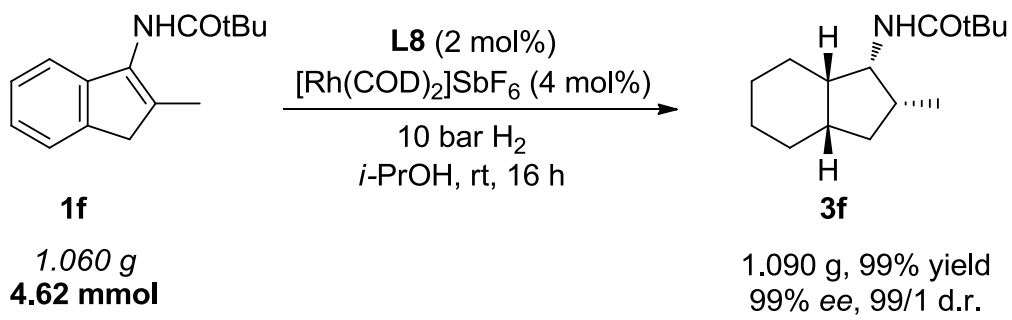

$\left[\mathrm{Rh}(\mathrm{COD})_{2}\right] \mathrm{SbF}_{6}(102 \mathrm{mg}, 0.18 \mathrm{mmol} 4 \mathrm{~mol} \%)$ was added to a glassed cylinder with a stirring bar. A stock solution of Pennphos $(2 \mathrm{mg} / \mathrm{ml})$ in $i$-PrOH $(16.5 \mathrm{ml}, 33 \mathrm{mg}, 2$ mol\%) was added to the cylinder and stirred for 30 min under argon. Substrate 1f $(1.060 \mathrm{~g}, 4.62 \mathrm{mmol})$ was dissolved in $10 \mathrm{ml} i-\mathrm{PrOH}$ and then was added to the cylinder under argon. The cylinder was placed in a high hydrogenation apparatus and then was purged three times with argon and three time with $\mathrm{H}_{2}$ before the indicated pressure (10 bar) was set. The reaction was stirred at room temperature for 16 hours before the $\mathrm{H}_{2}$ pressure was released and the solvent was removed in vacuo. The crude product was filtered through on a short plug of silica to yield the product $\mathbf{3 f}$ (1.090 g, 99\% yield), d.r and $e e$ value was determined by GC using a chiral stationary phase. 


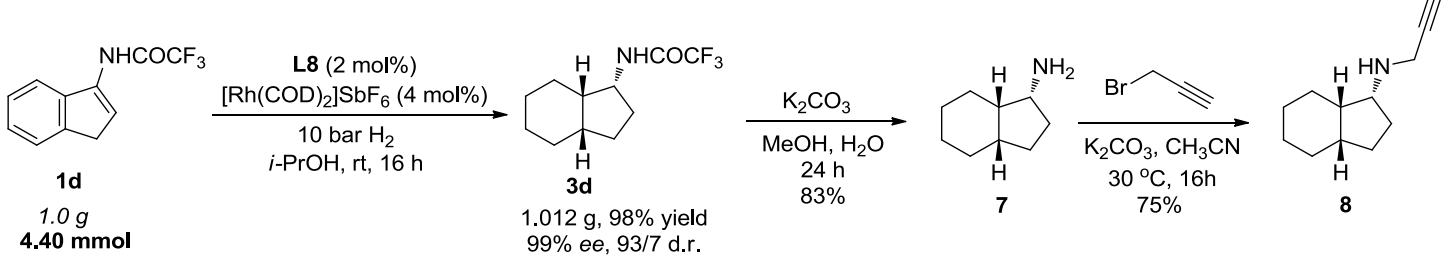

Step 1: Hydrogenation was performed using the same procedure as the above case.

Step 2: Deprotection: To a solution of 3d (235 mg, $1.0 \mathrm{mmol})$ in $10 \mathrm{ml}$ $\mathrm{MeOH} / \mathrm{H}_{2} \mathrm{O}(1: 1)$ was add $\mathrm{K}_{2} \mathrm{CO}_{3}(276 \mathrm{mg}, 2.0 \mathrm{~mol})$ and the mixture was stirred at ambient temperature for $24 \mathrm{~h}$ before the volatles were removed in vacuo. The residue was partitioned between $\mathrm{CH}_{2} \mathrm{Cl}_{2}$ and saturated aqueous $\mathrm{NaHCO}_{3}$. The organic layer was separated and washed with brine, died over $\mathrm{Na}_{2} \mathrm{SO}_{4}$ and concentrated to give the product 7 as yellowish oil (115 mg, 83\% yield).

Step 3: Alkylation: $\mathrm{K}_{2} \mathrm{CO}_{3}(120 \mathrm{mg}, 0.84 \mathrm{mmol}, 1.2$ eq.) was added to a solution of 7 (100mg, $0.72 \mathrm{mmol})$ in $5 \mathrm{ml}$ acetonitrile. Propargyl bromide $(150 \mathrm{mg}$, $80 \%$ w.t. in toluene, $0.72 \mathrm{mmol}$ ) was added to the reaction mixture with stirring at $30{ }^{\circ} \mathrm{C}$. After stirring at $30{ }^{\circ} \mathrm{C}$ for $12 \mathrm{~h}$, potassium carbonate was filtered off, whereupon acetonitrile was removed, and the residue was purified by column chromatography (EtOAc: pentane $=1: 1$ ). The product 8 was obtained as yellowish oil $(95 \mathrm{mg}, 75 \%$ yield).

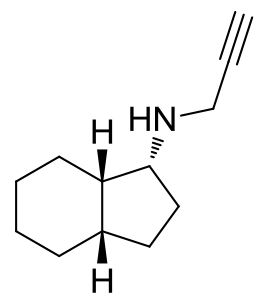

${ }^{1} \mathrm{H}$ NMR (400 MHz, Chloroform- $d$ ) $\delta 3.44(\mathrm{~d}, \mathrm{~J}=2.5 \mathrm{~Hz}, 2 \mathrm{H})$, $3.29(\mathrm{td}, \mathrm{J}=9.0,5.6 \mathrm{~Hz}, 1 \mathrm{H}), 2.57(\mathrm{~s}, 1 \mathrm{H}), 2.23(\mathrm{t}, \mathrm{J}=2.5 \mathrm{~Hz}, 1 \mathrm{H})$, $2.14-1.87(\mathrm{~m}, 3 \mathrm{H}), 1.76-1.60(\mathrm{~m}, 2 \mathrm{H}), 1.60-1.42(\mathrm{~m}, 6 \mathrm{H})$, $1.41-1.28(\mathrm{~m}, 1 \mathrm{H}), 1.20-0.97(\mathrm{~m}, 2 \mathrm{H}) .{ }^{13} \mathrm{C}$ NMR $(100 \mathrm{MHz}$, Chloroform- $d$ ) $\delta 81.81,71.73,61.94,41.12,37.17,37.12,28.84,27.11,25.16$, 24.18, 21.47, 21.00. $[\alpha]_{\mathrm{D}}{ }^{29}=14.0\left(\mathrm{c}=0.2, \mathrm{CHCl}_{3}\right)$. HRMS-ESI: Found $[\mathrm{M}+\mathrm{Na}]^{+}=$ 200.1417; $\mathrm{C}_{16} \mathrm{H}_{28} \mathrm{NO}_{2} \mathrm{Na}$ requires 200.1415. 


\section{Additional experiments and HR-TEM images of in-situ generated Rh- nanoparticles.}

\section{Doping experiment with benzothiophene}

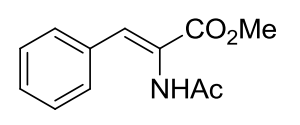

$4 \mathbf{a}$

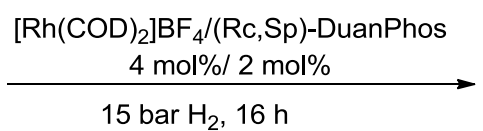

15 bar $\mathrm{H}_{2}, 16 \mathrm{~h}$

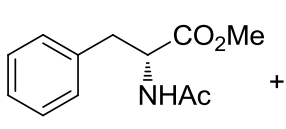

$5 \mathbf{a}$

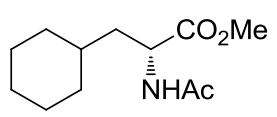

$6 \mathbf{a}$

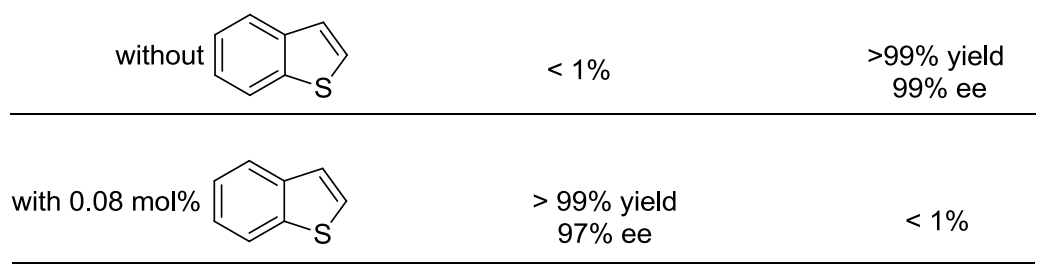

Investigations concerning the stability of homogeneous $\mathrm{Rh} /$ diphosphine catalyst under the hydrogenation conditions.

(a)

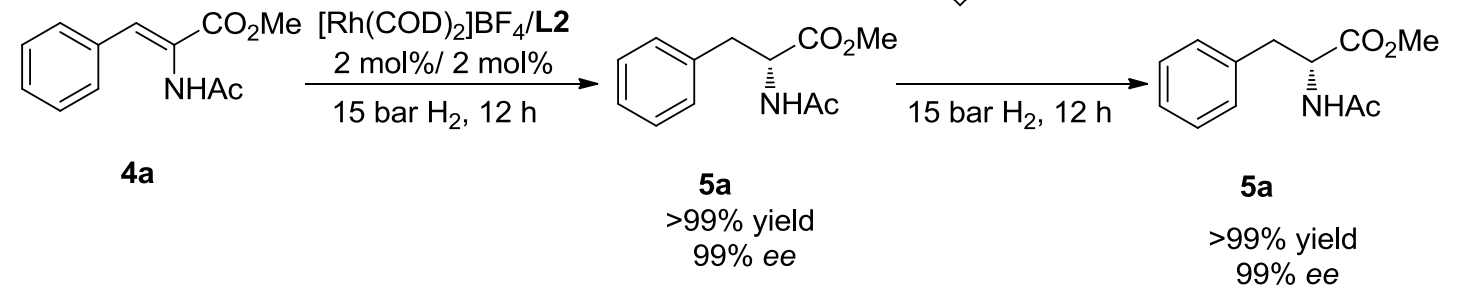

$\left[\mathrm{Rh}(\mathrm{COD})_{2}\right] \mathrm{BF}_{4}$ was used in a ratio of 1:1 with diphosphine ligand $\mathbf{L} \mathbf{2}$ to form the homogeneous $\mathrm{Rh} /$ diphosphine catalyst for the asymmetric hydrogenation of olefin. After $12 \mathrm{~h}$, the olefin hydrogenation was completed and another 1 equivalent of substrate $4 \mathbf{a}$ was added to the reaction mixture and then subjected to hydrogenation again. No aromatic hydrogenation product was observed, and the enantioselectivity for the olefin hydrogenation was not affected (99\% ee). The results indicate that the homogeneous $\mathrm{Rh}$ /diphosine catalyst is stable under olefin hydrogenation conditions, and no in-situ formation of Rh-nanoparticles occurred. 
(b)

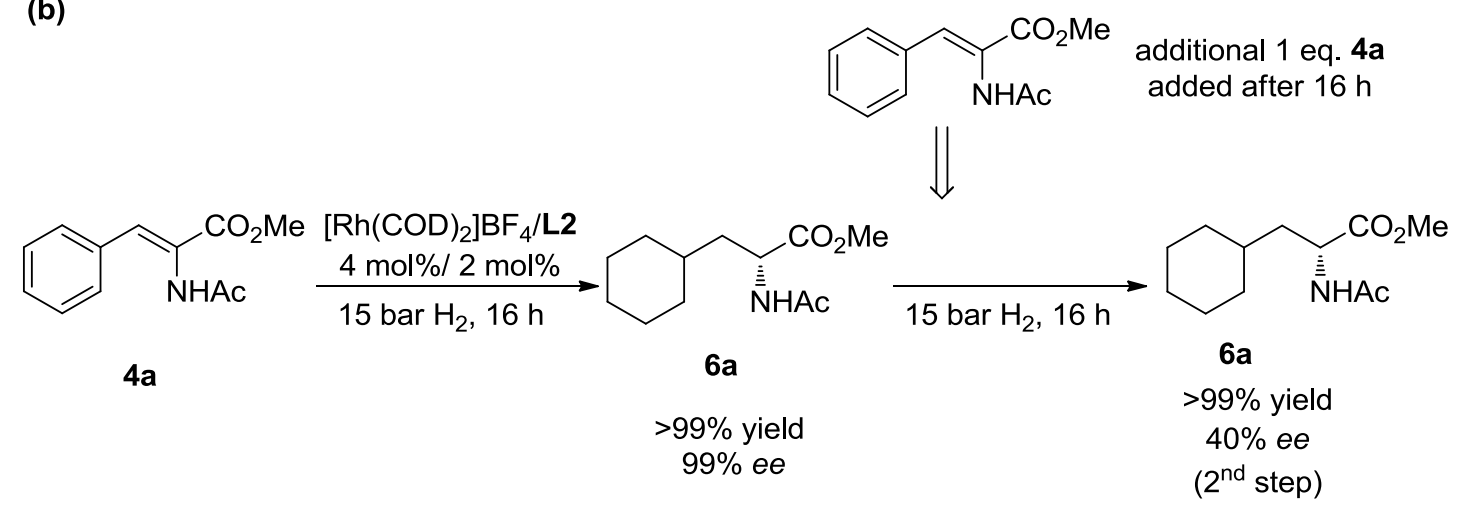

$\left[\mathrm{Rh}(\mathrm{COD})_{2}\right] \mathrm{BF}_{4}$ was used in a ratio of 2:1 with diphosphine ligand $\mathbf{L 2}$ for the hydrogenation of both olefin and phenyl ring. After $16 \mathrm{~h}$, a full conversion to the complete hydrogenation product 5a (99\% ee) was obtained. Another 1 equivalent of substrate $4 \mathbf{a}$ was added to the reaction mixture and then subjected to hydrogenation for $16 \mathrm{~h}$. In this case, the complete hydrogenation of the second portion of substrate 4a was observed. Lower ee (40\% ee) was obtained for the second hydrogenation might due to the competition hydrogenation of olefin by the in-situ generated Rhnanoparticles.

(c)<smiles>CC(=O)N/C(=C\c1ccccc1)C(C)=O</smiles>
additional 1 eq. $\mathbf{4 a}$ added after $16 \mathrm{~h}$<smiles>O=C([O-])NC(=O)/C(=C/c1ccccc1)C(=O)O</smiles>

4a

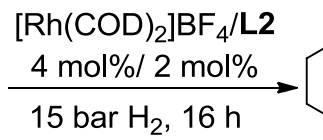
5 bar $\mathrm{H}_{2}, 16 \mathrm{~h}$<smiles>C1CCCCC1</smiles>

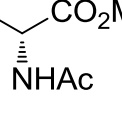

$6 a$

$>99 \%$ yield

$99 \%$ ee
Filtrate without
Rh-nanoparticle<smiles>CC(=O)NC(Cc1ccccc1)C(C)=O</smiles>

$5 a$

$>99 \%$ yield

$99 \%$ ee

In this experiment, the same conditions as (b) were applied for the first step. After $16 \mathrm{~h}$, hydrogen atmosphere was released and the sample was left stand for $1 \mathrm{~h}$ under argon (aggregation of nanoparticles). The aggregated Rh-nanoparticles were then removed from the mixture by filtration under argon. Another 1 equivalent of $\mathbf{4 a}$ was added to the filtrate and subjected to the hydrogenation conditions. In this case, only olefin hydrogenation was observed for the second portion of substrate $\mathbf{4 a}$, and the 
enantioselectivity was not affected (99\% ee of 5a). Although not conclusive, the same level of ee $(99 \%)$ was retained in the second olefin hydrogenation indicates that the in-situ generated Rh-nanoparticles might not influence the stability of homogeneous $\mathrm{Rh} /$ diphosphine catalyst.

HR-TEM images of in-situ generated Rh-nanoparticles from complete hydrogenation of $1 \mathrm{a}$.

Transmission Electron Microscopy. TEM images were obtained on a JEOL JEM2100F microscope operated at $200 \mathrm{kV}$ (Cs $1.0 \mathrm{~mm}$, point resolution $0.23 \mathrm{~nm}$ ) with a Gatan Ultrascan 1000 CCD camera (resolution 2,048 $\times 2,048$ pixels, pixel size $14 \mu \mathrm{m})$.

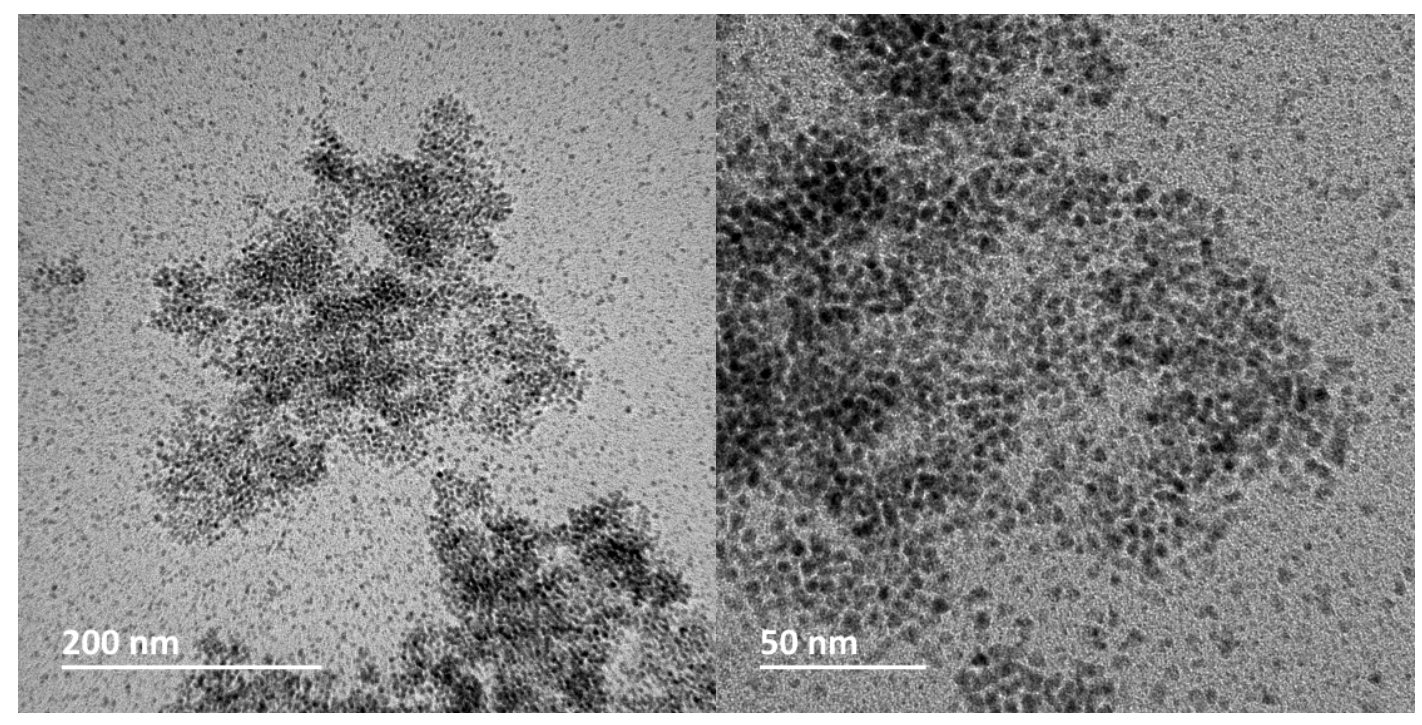

Figure S3. TEM images of Rh nanoparticles showing size of 3-5 nm. 
9. ${ }^{1} \mathrm{H},{ }^{13} \mathrm{C}$ and ${ }^{1} \mathrm{~F}$ NMR spectra for new compounds

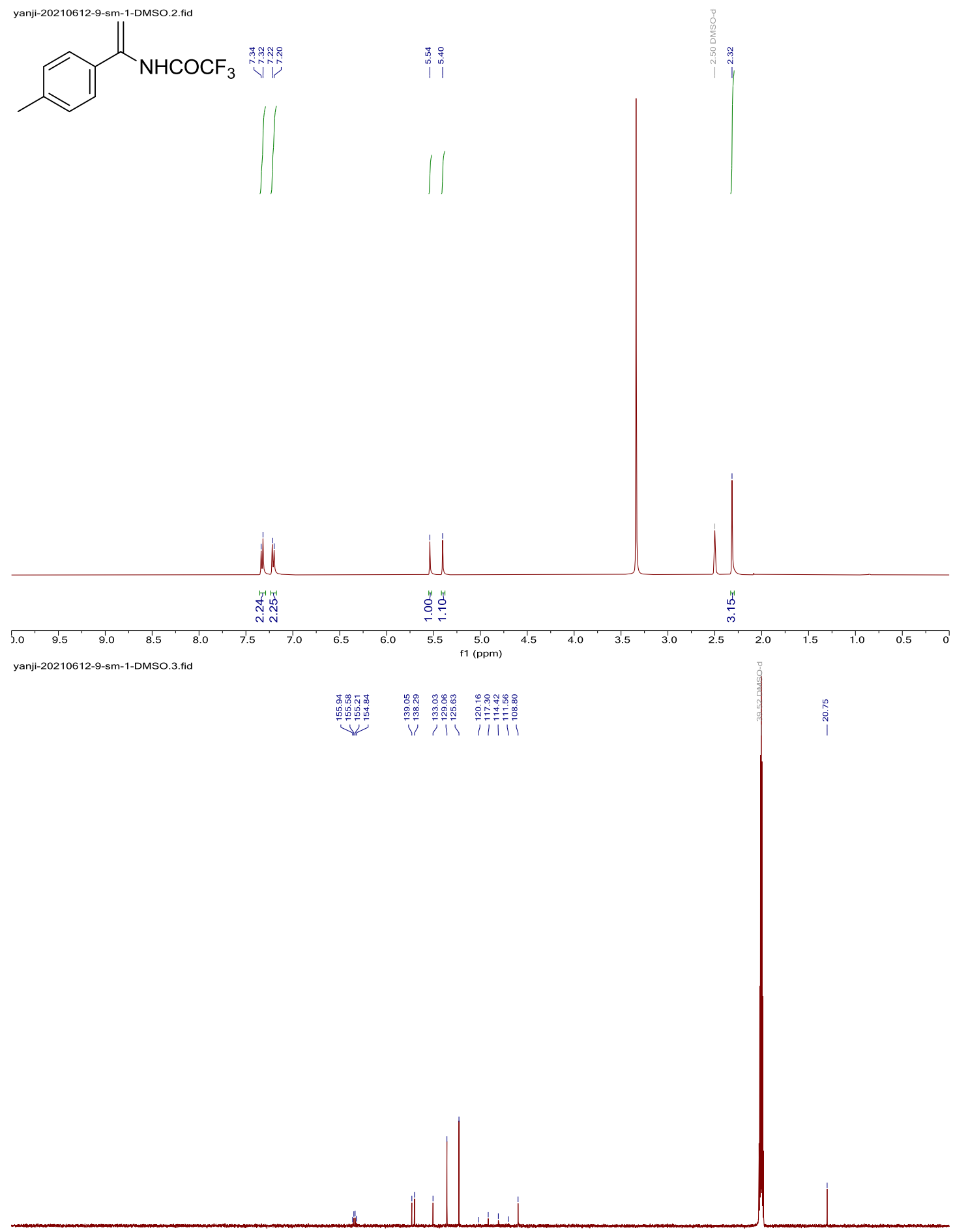

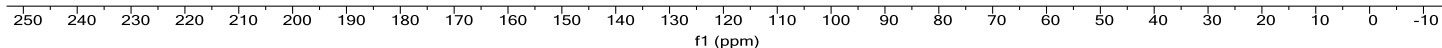




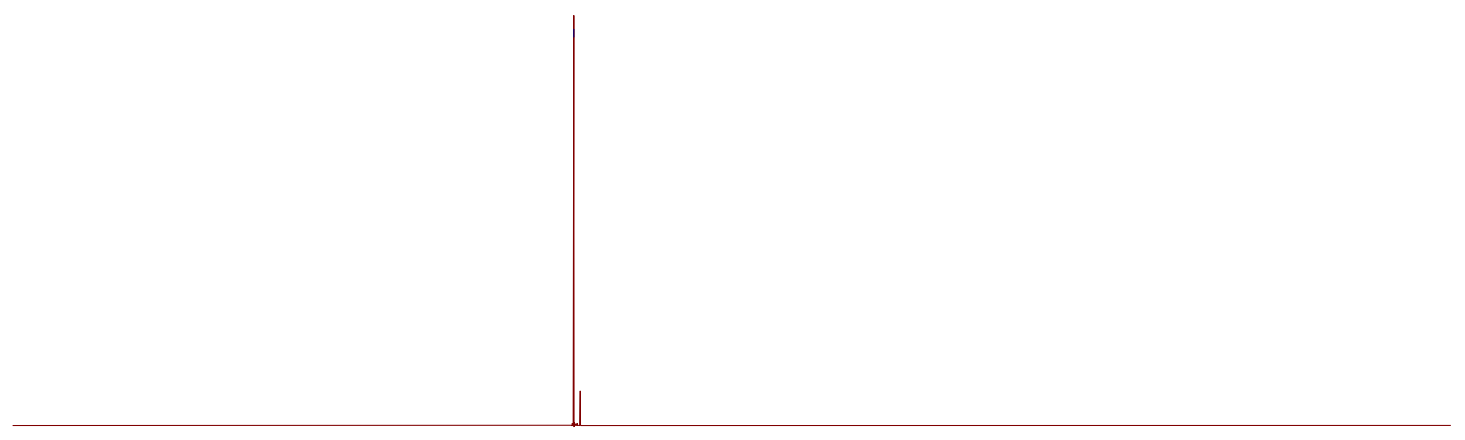

$\begin{array}{lllllllllllllllllllllllllllll}10 & 0 & -10 & -20 & -30 & -40 & -50 & -60 & -70 & -80 & -90 & -100 & -110 & -120 & -130 & -140 & -150 & -160 & -170 & -180 & -190 & -200 & -210 & 1\end{array}$ 
<smiles>C=C(NC(=O)C(F)(F)F)c1cccc(C)c1</smiles>

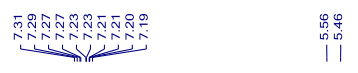

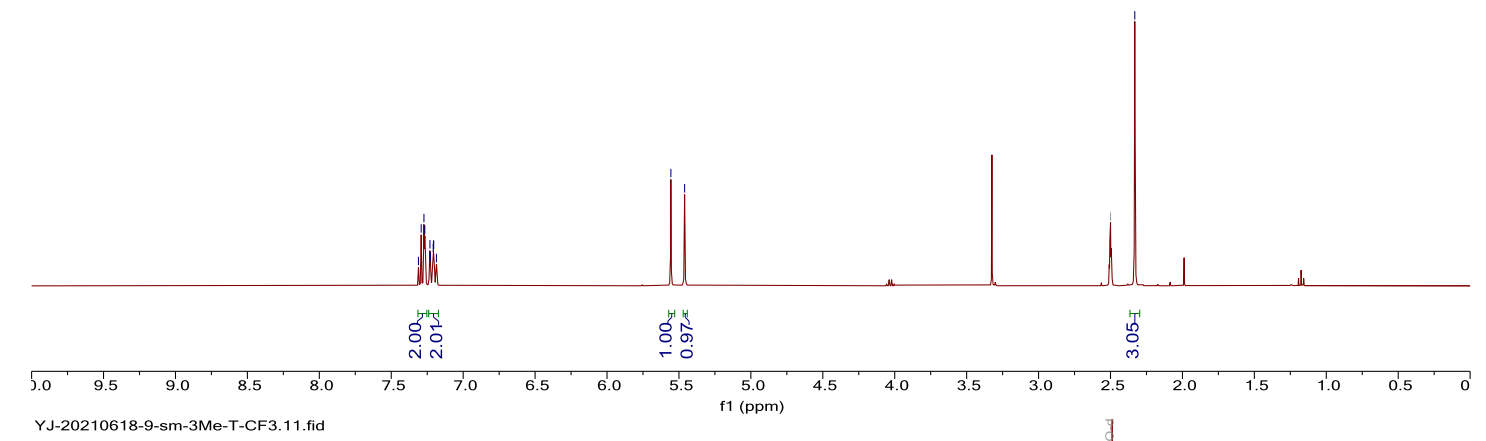

YJ-20210618-9-sm-3Me-T-CF3.11.fid

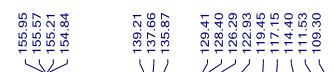

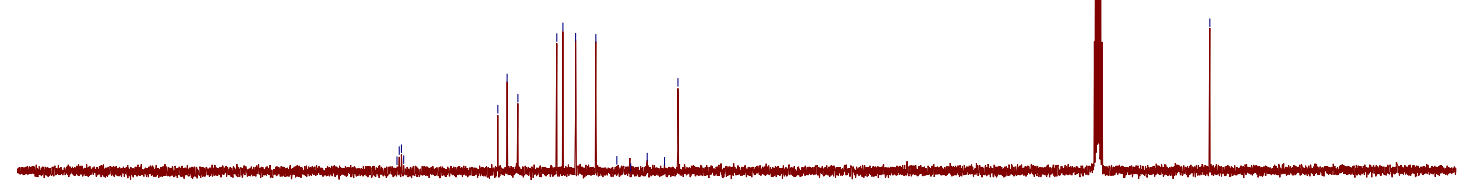

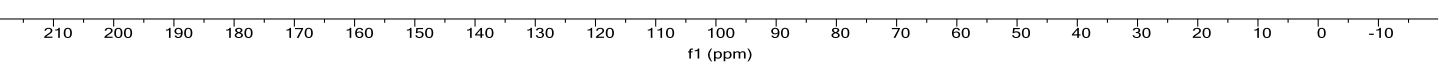




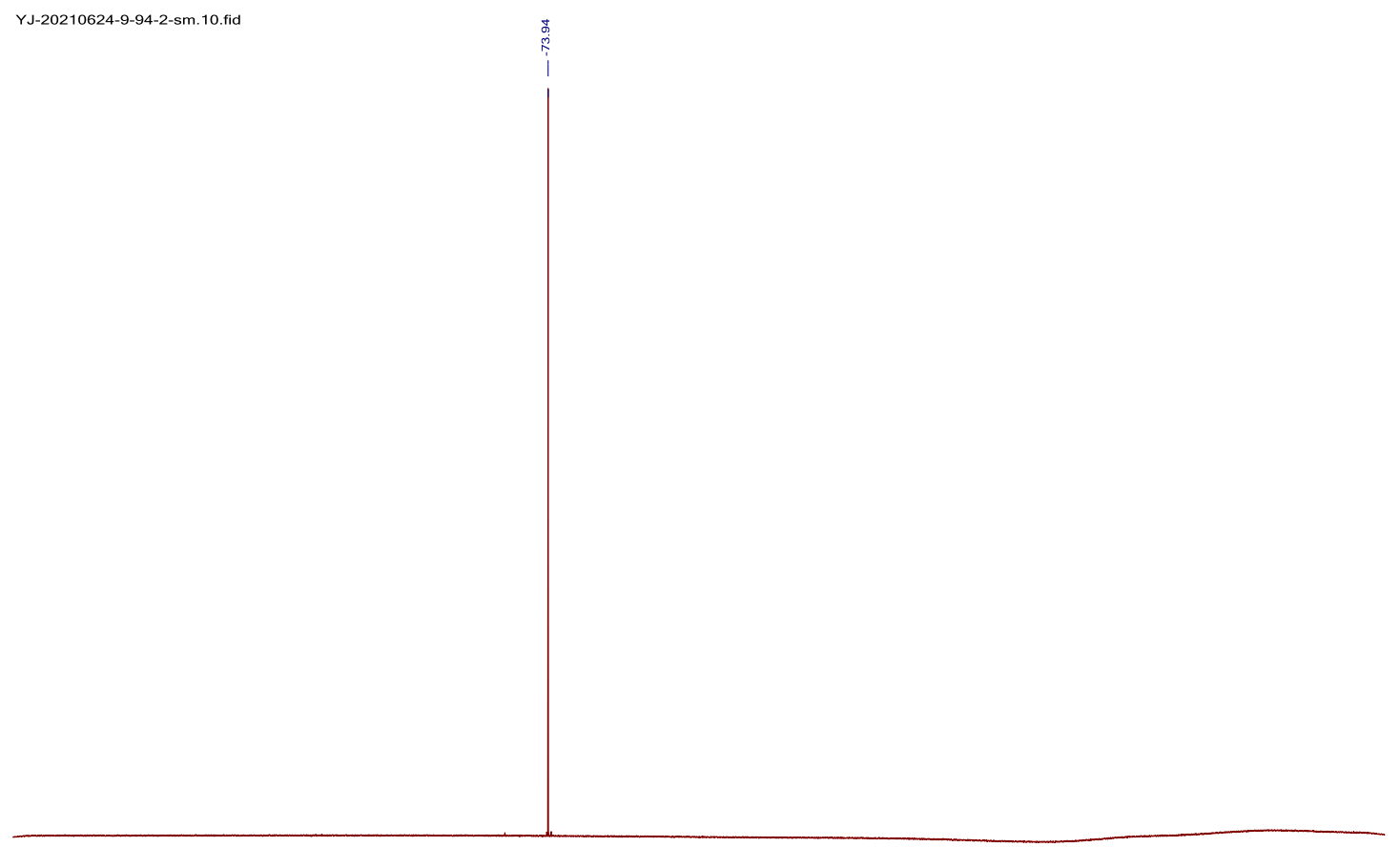

\begin{tabular}{llllllllllllllllllllllll}
10 & 0 & -10 & -20 & -30 & -40 & -50 & -60 & -70 & -80 & -90 & -100 & -110 & -120 & -130 & -140 & -150 & -160 & -170 & -180 & -190 & -200 & -210 & 1 \\
\hline
\end{tabular} 

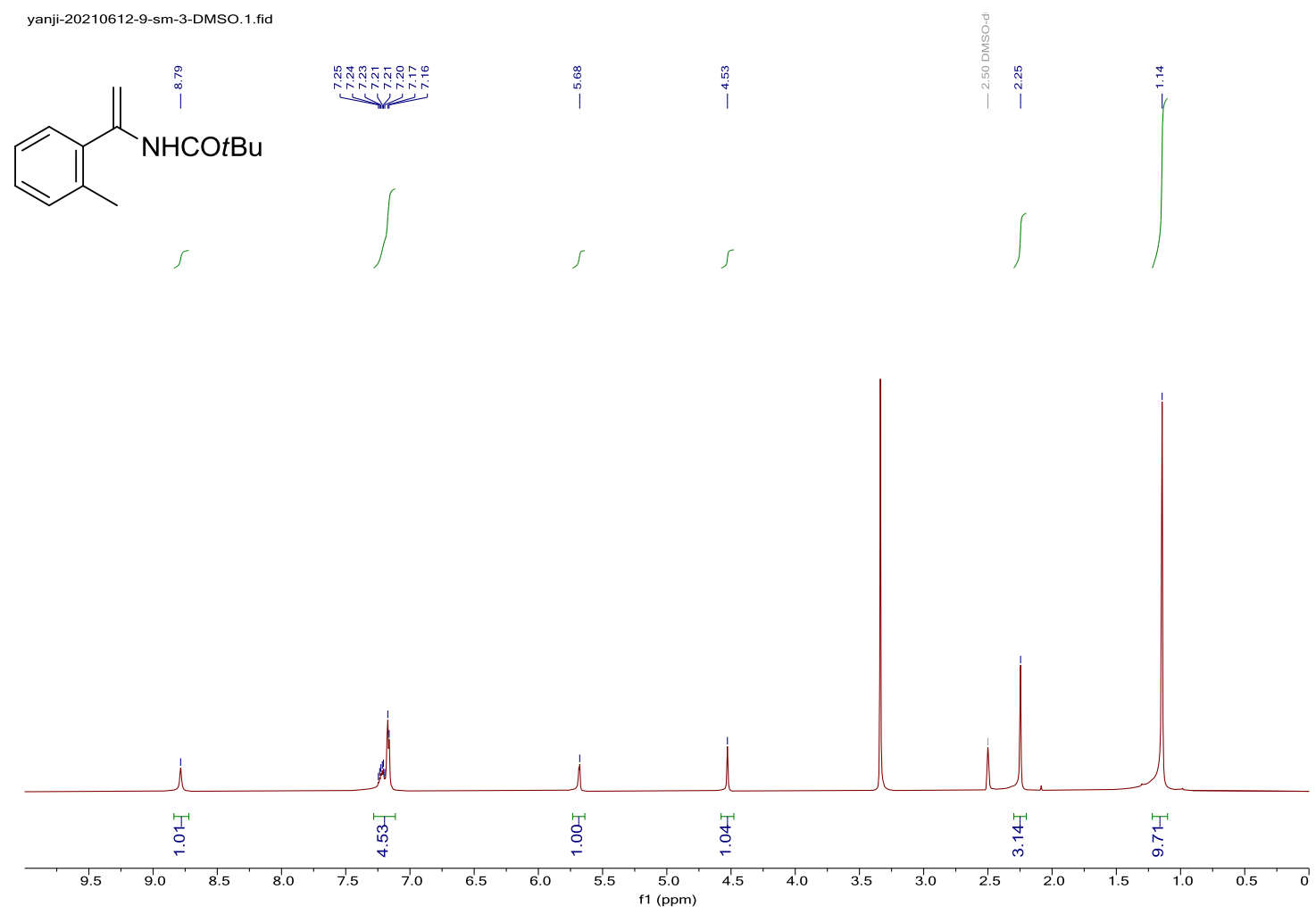

yanji-20210612-9-sm-3-DMSO.2.fid

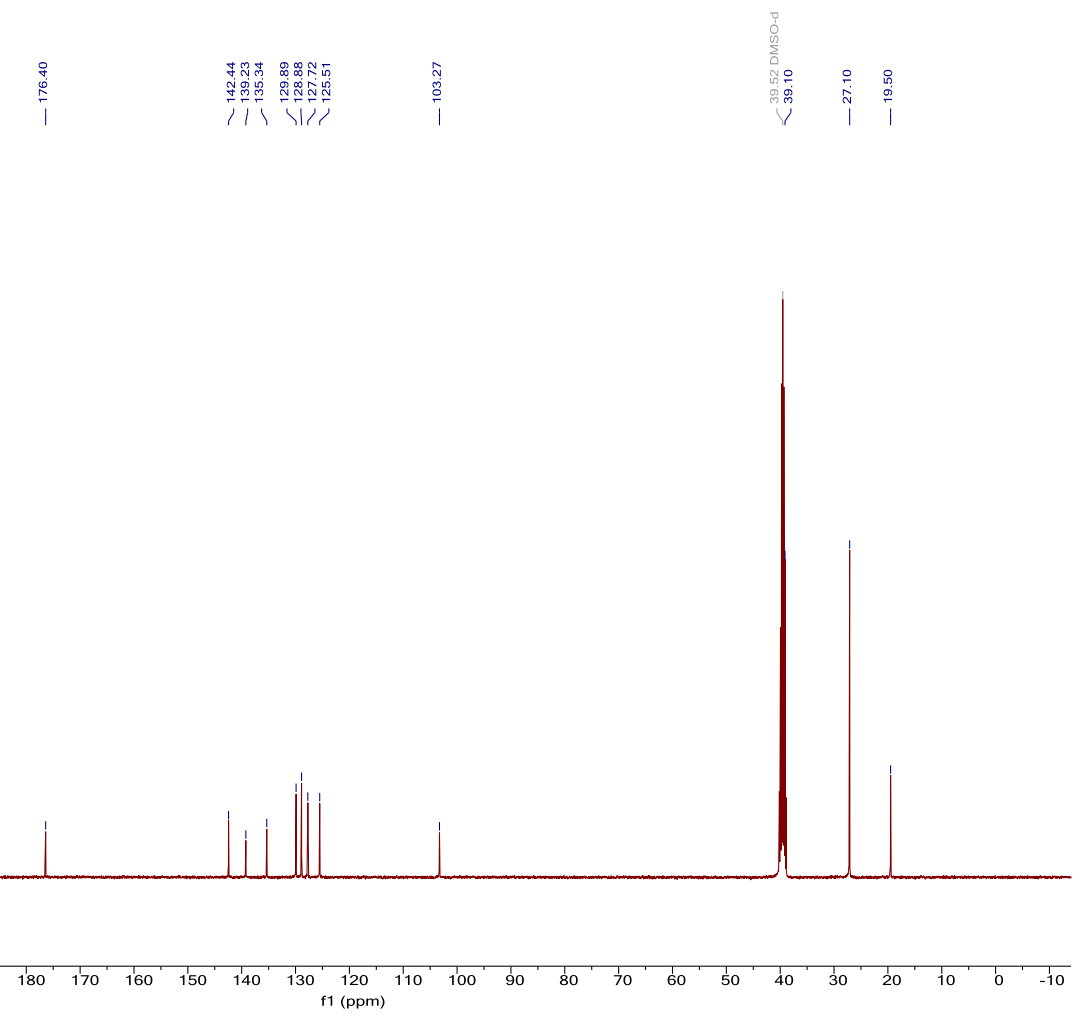

S39 


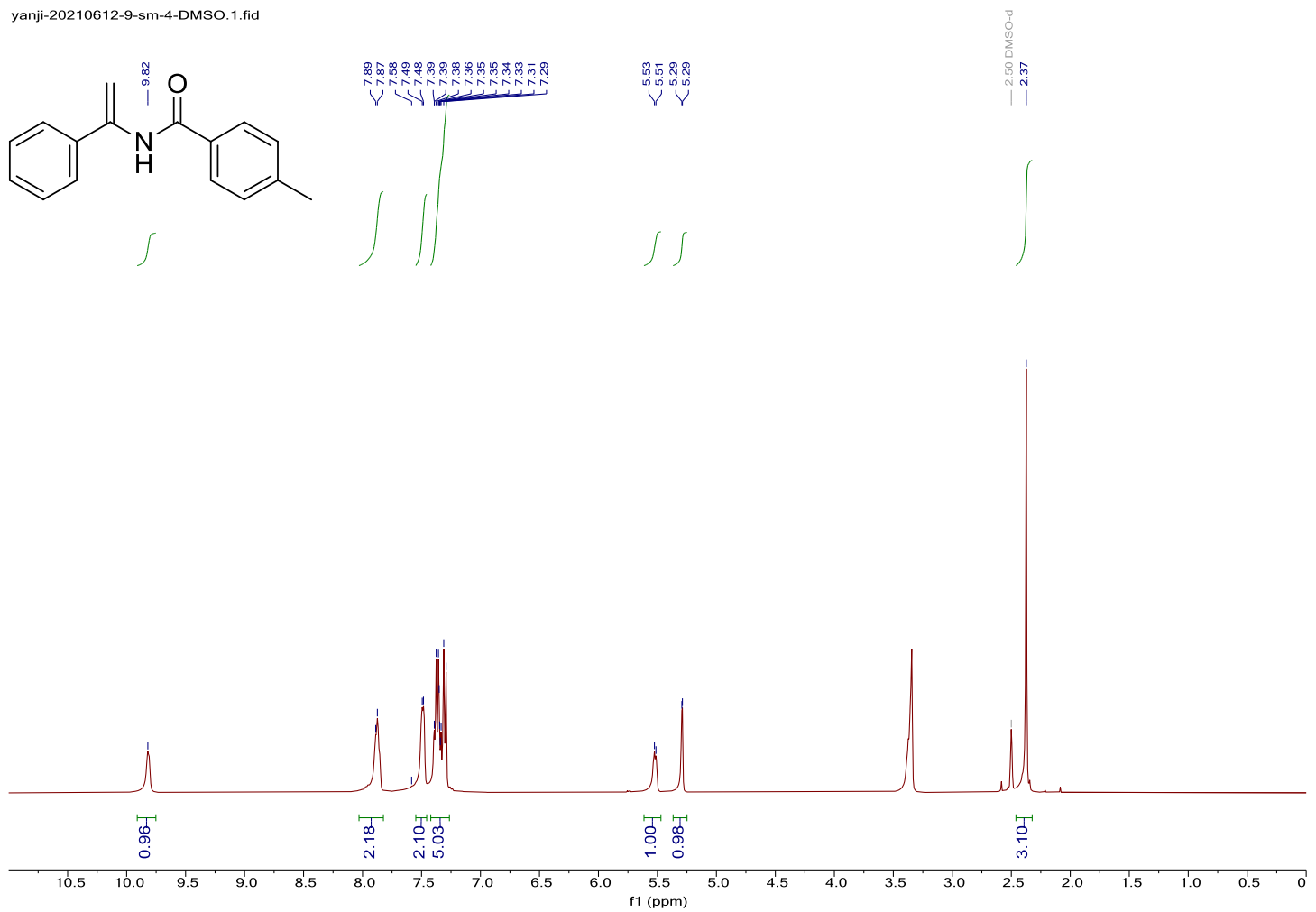

yanji-20210612-9-sm-4-DMSO.2.fid
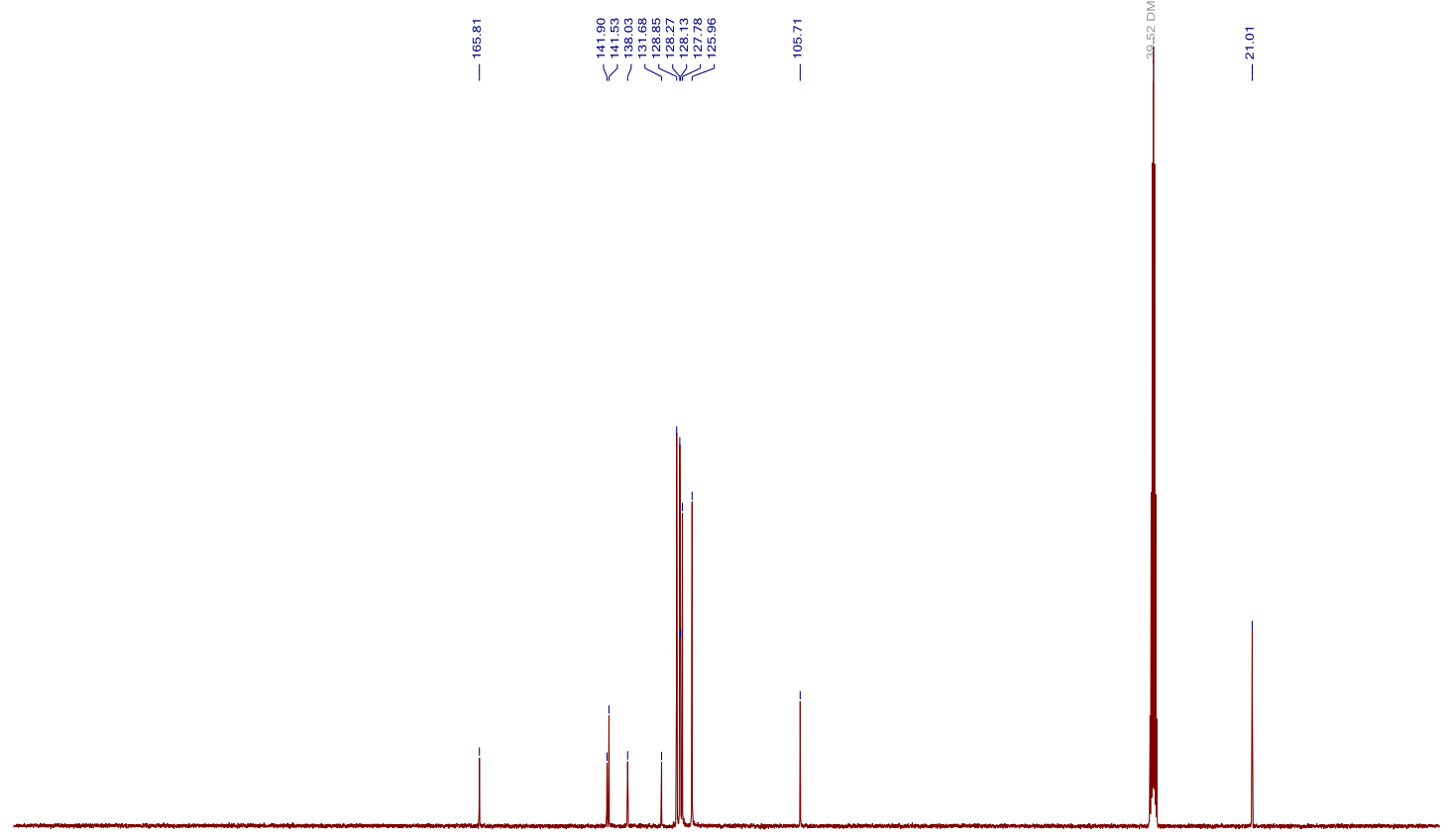

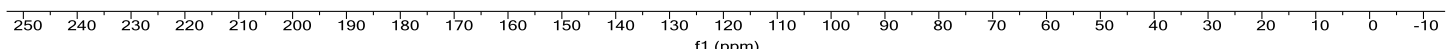


YJ-20210624-9-4-3-sm.10.fid

$\underset{\substack{\infty \\ \infty \\ \infty}}{i}$

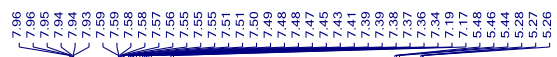

$\overbrace{1}$
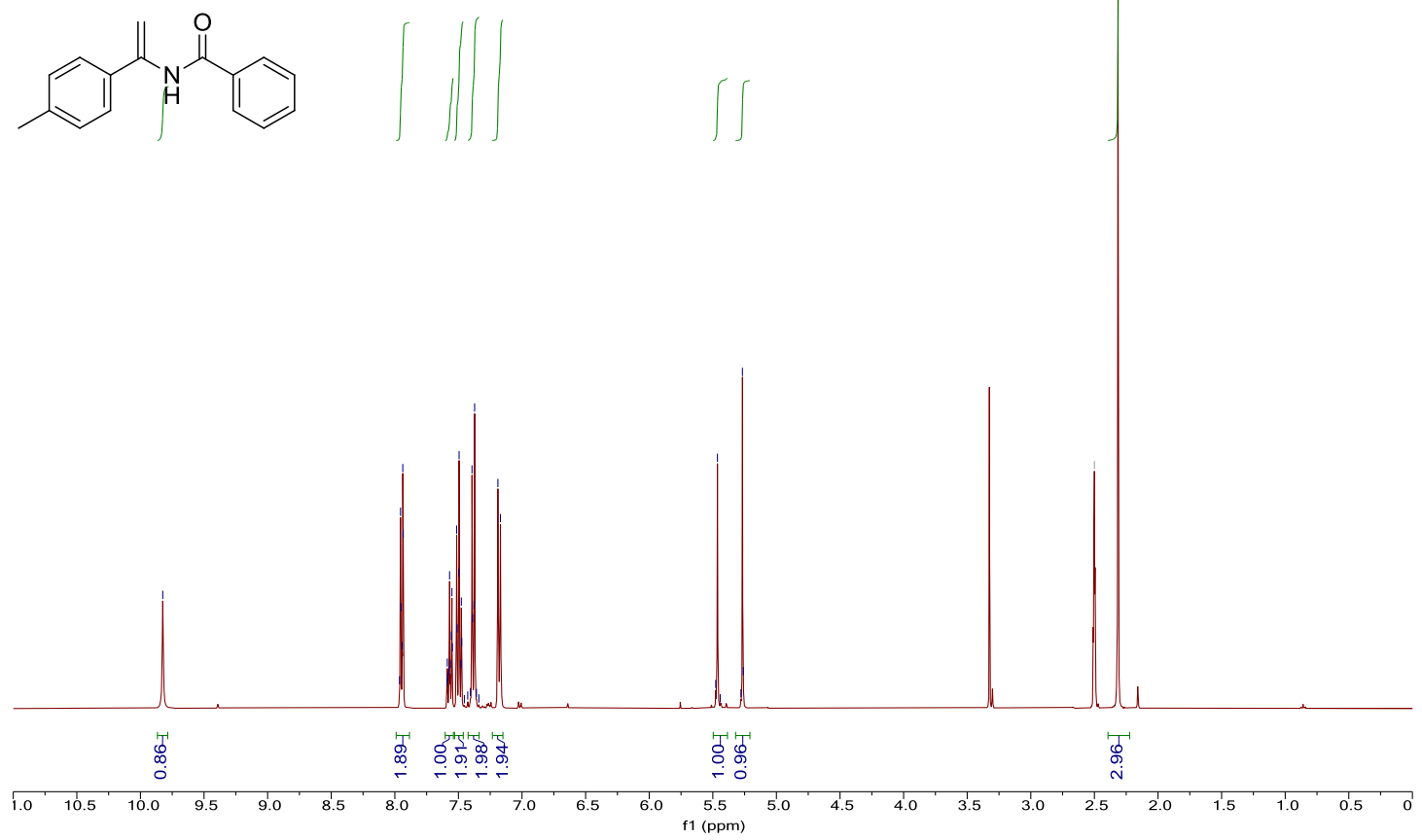

yanji-20210612-9-sm-5-DMSO.2.fid

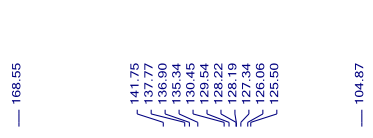

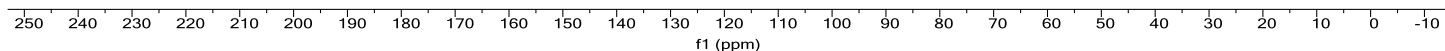


hwu-210621-206-82fsmdmso.10.fid
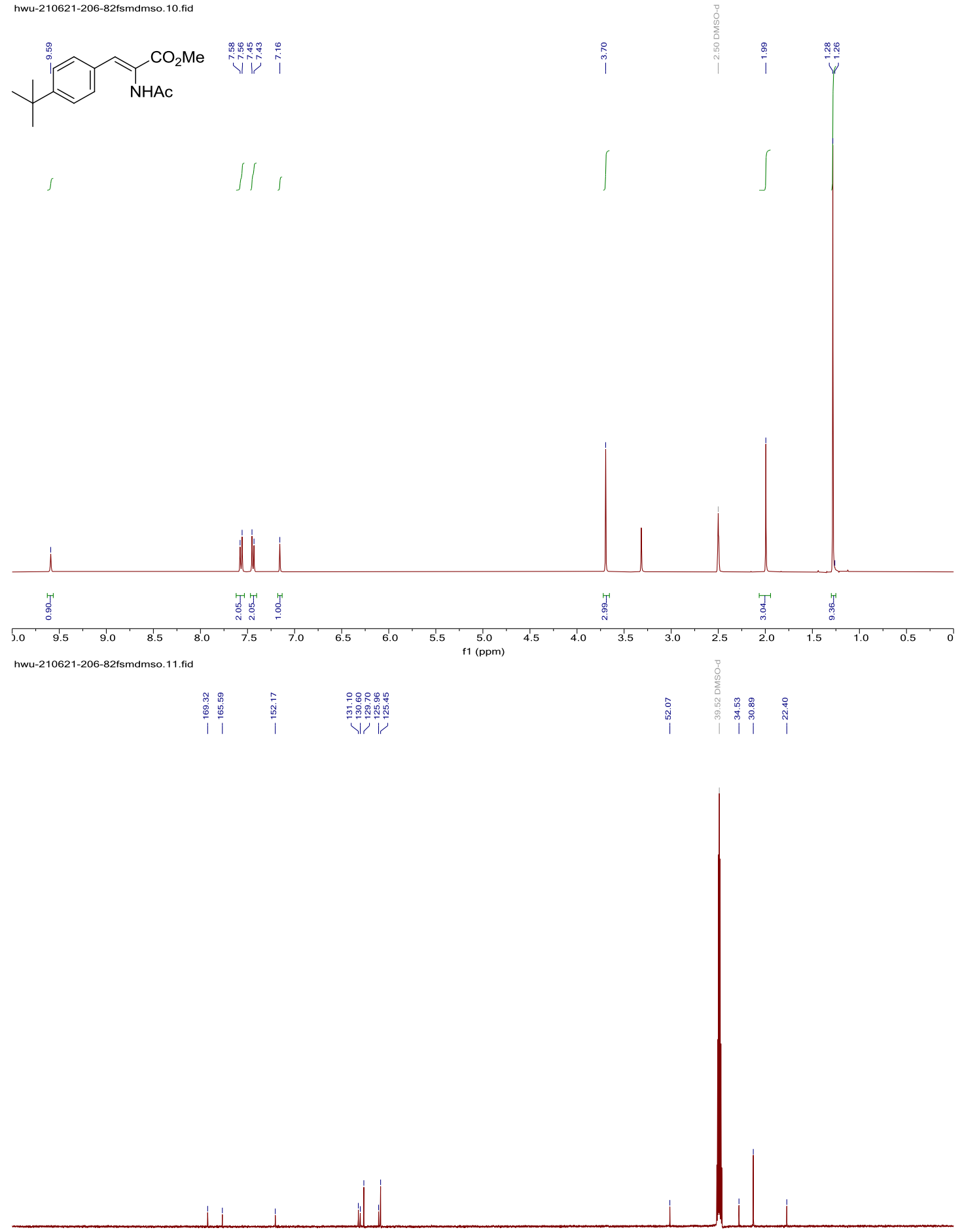

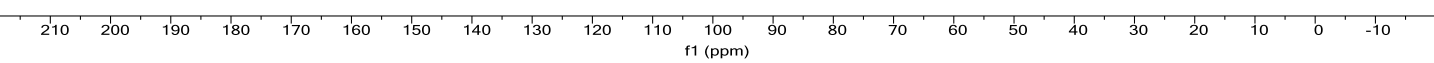



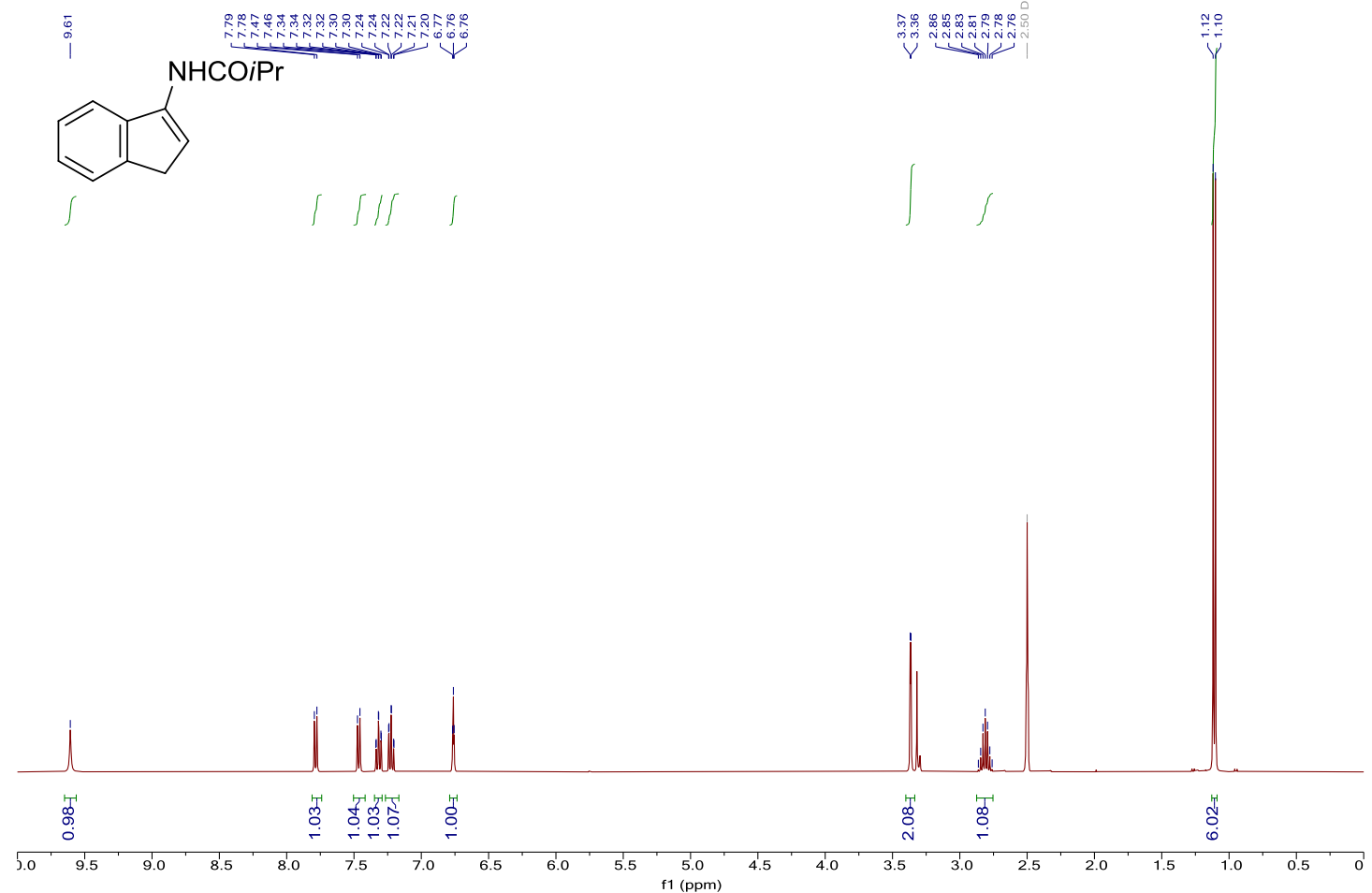

YJ-20210613-9-sm-10-DMSO.11.fid

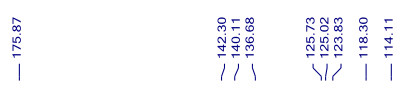
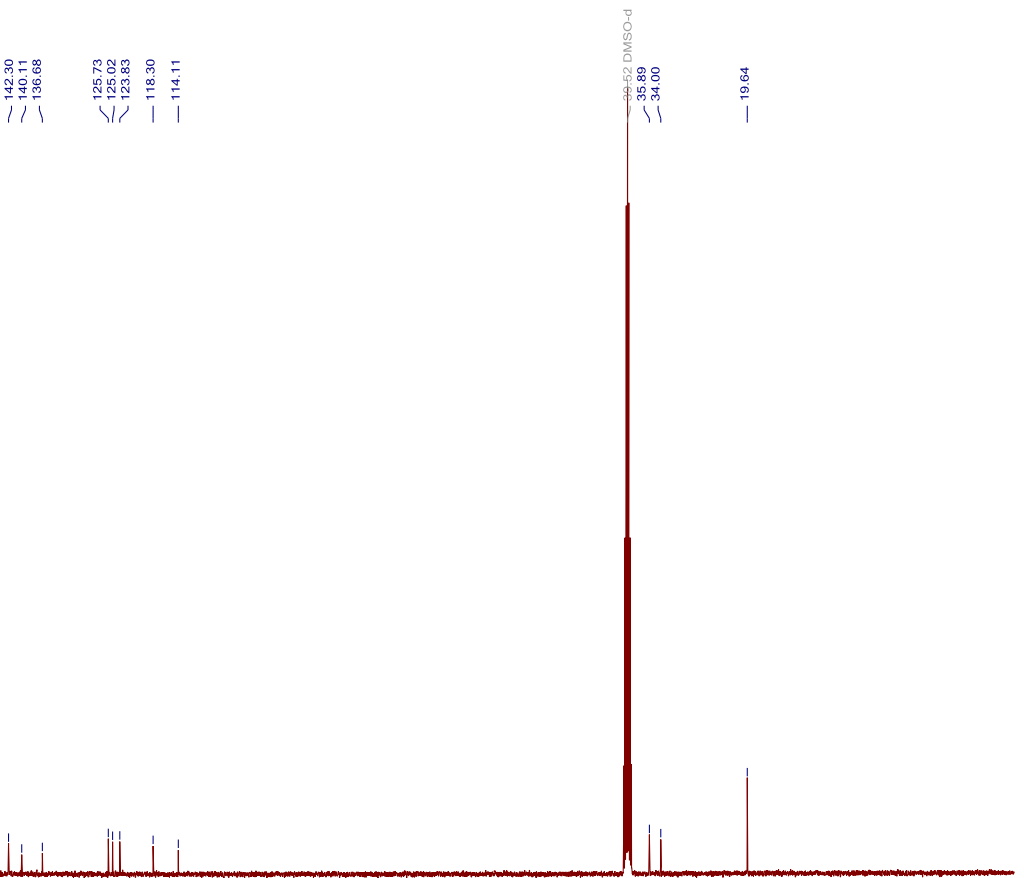

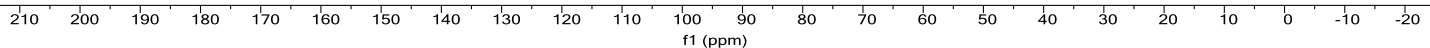



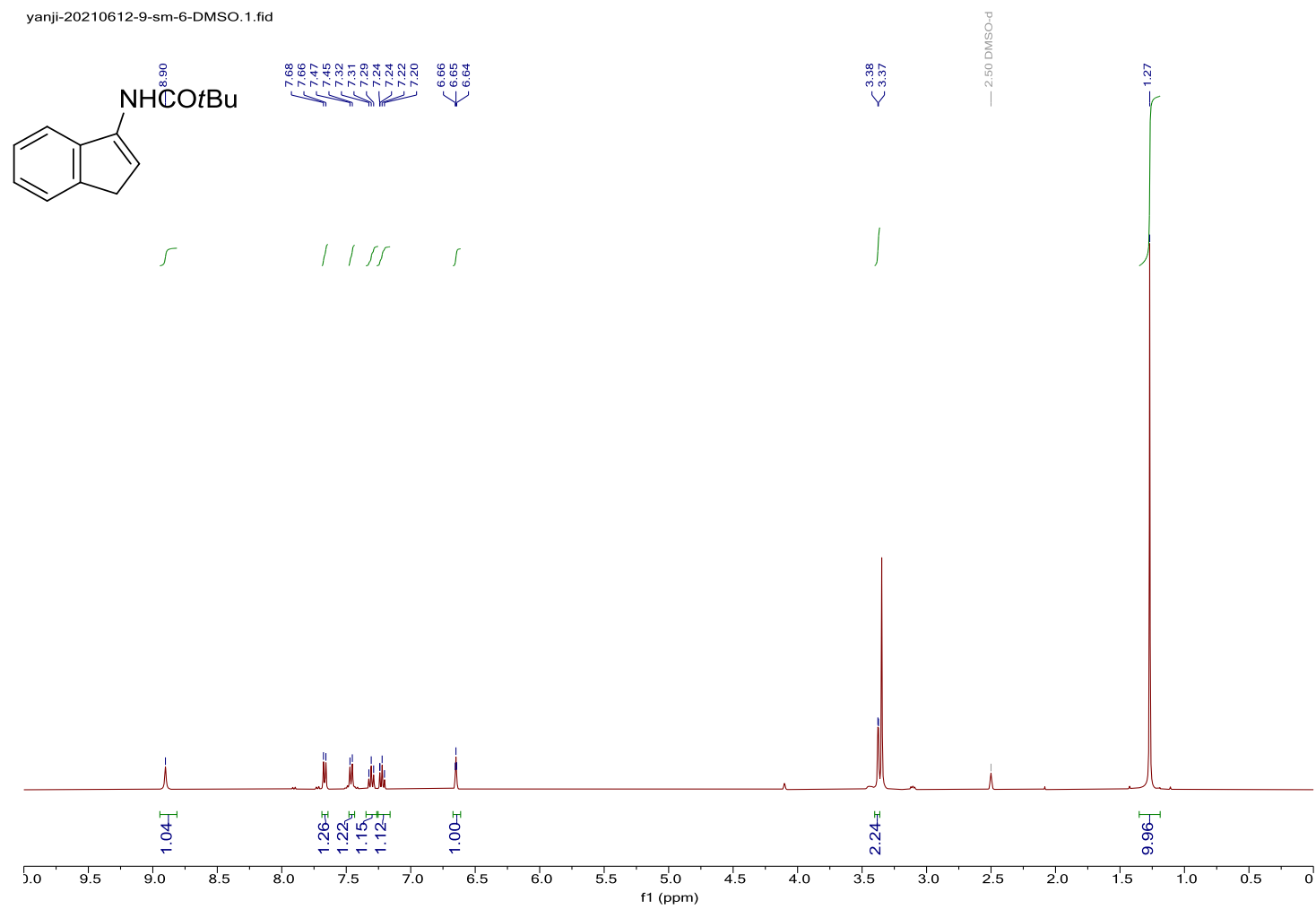

yanji-20210612-9-sm-6-DMSO.2.fid
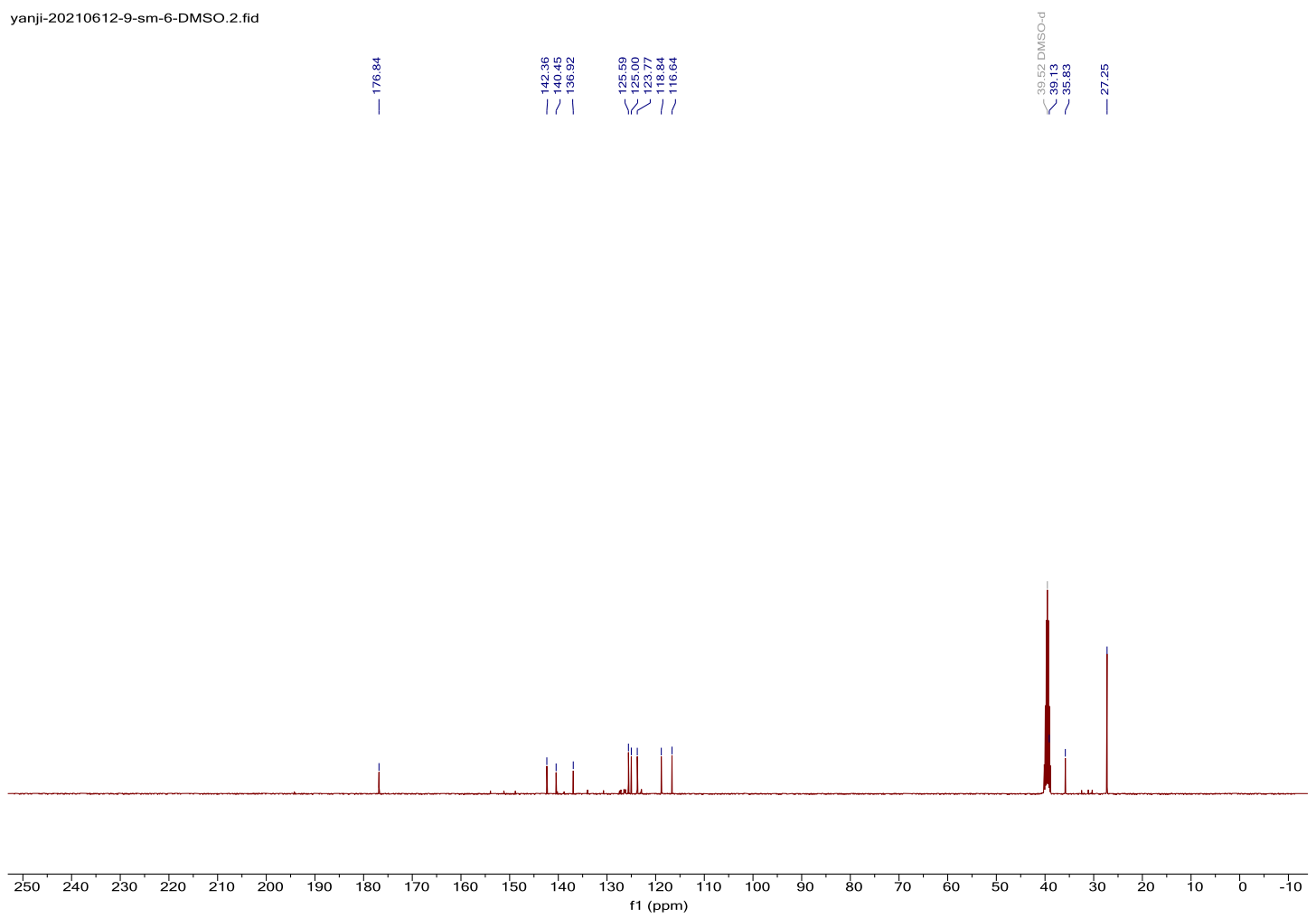

S44 
hwu-210608-206-79asm.10.fid
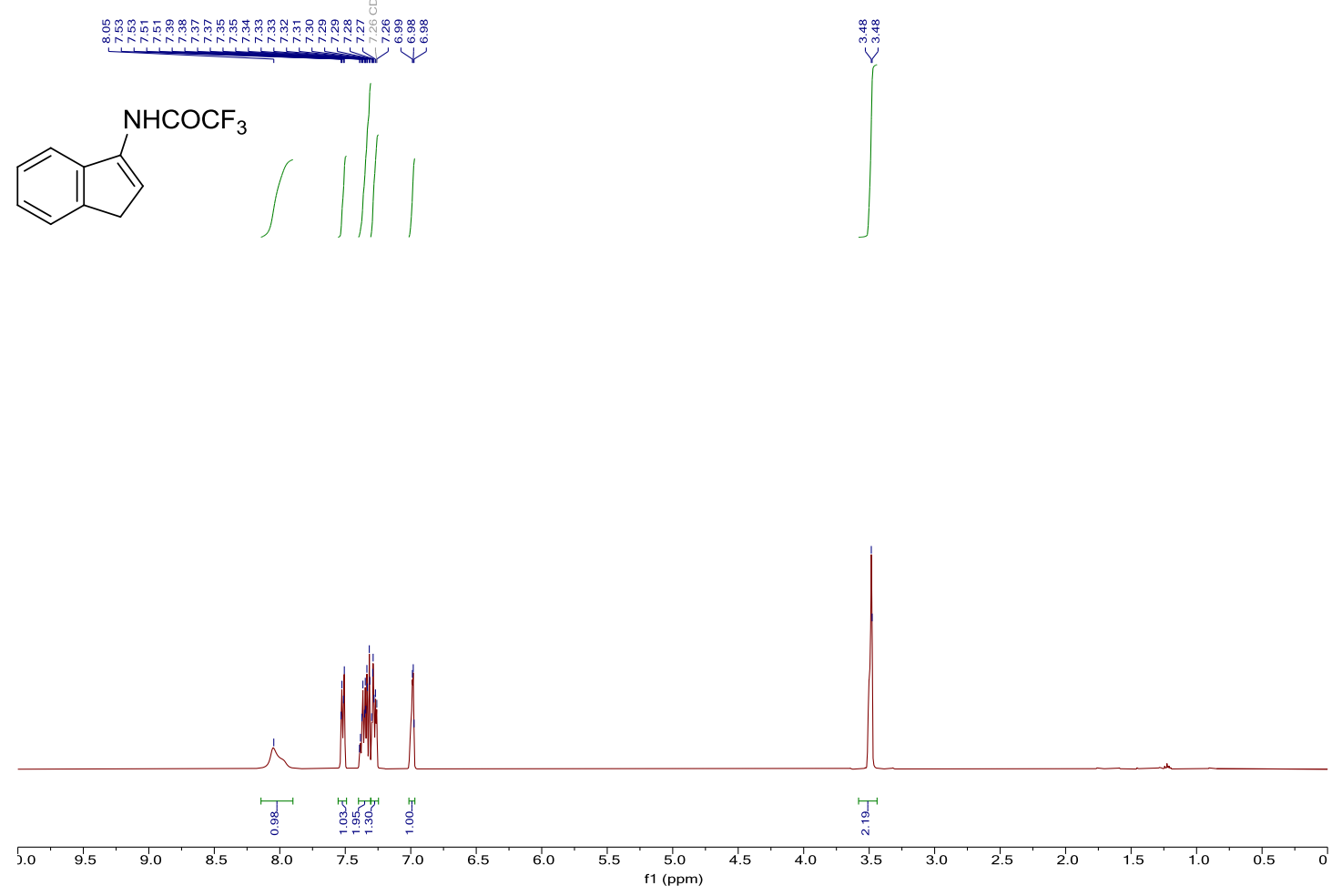

hwu-210608-206-79asm.12.fid

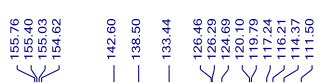

VI,

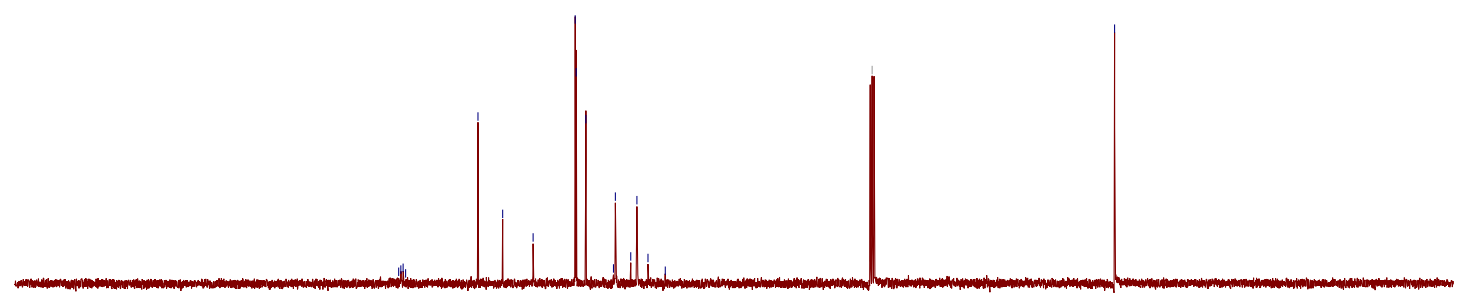

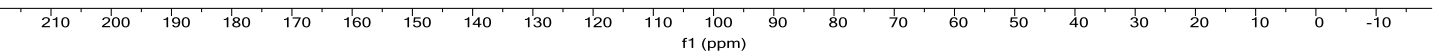


hwu-210608-206-79asm.11.fid

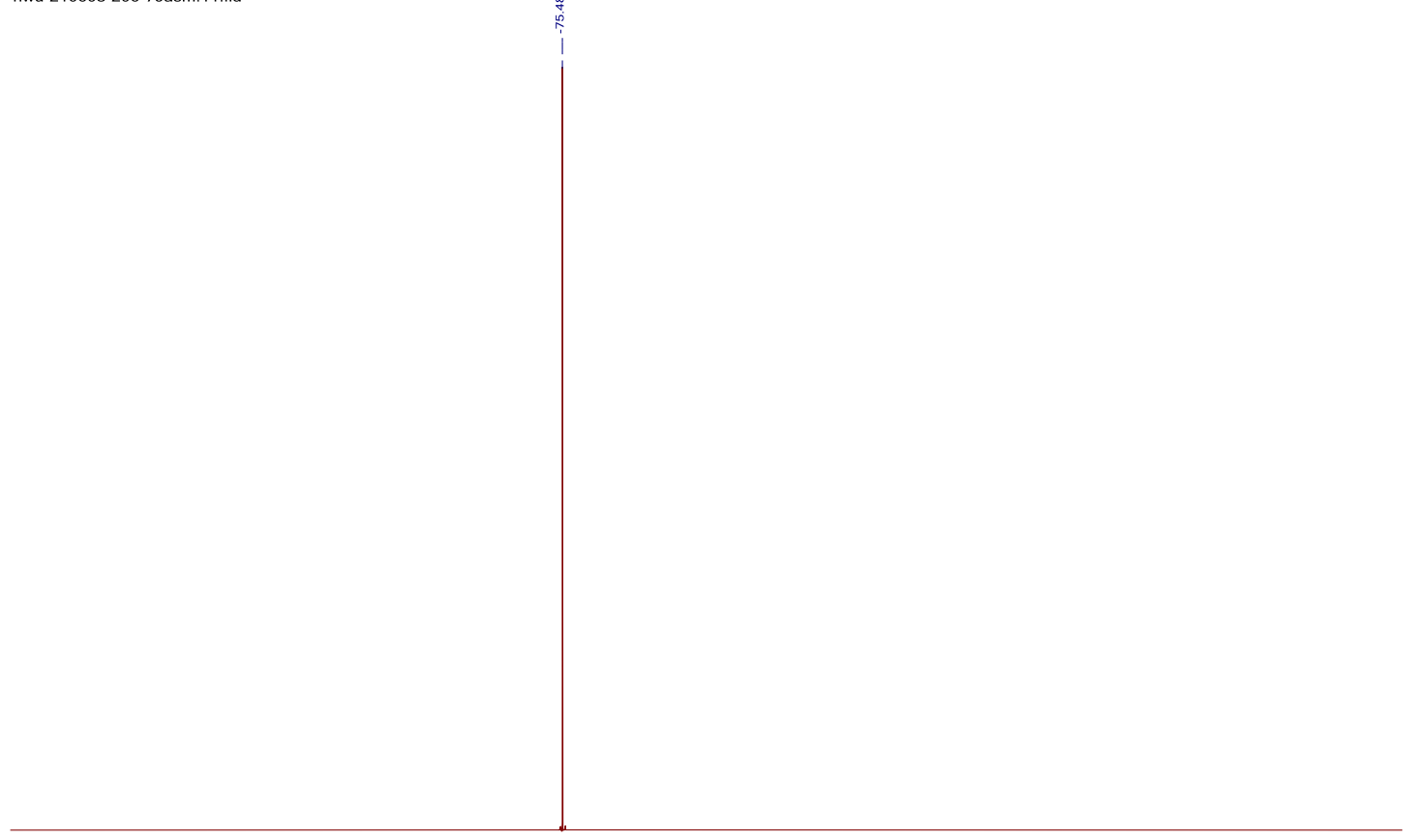

\begin{tabular}{llllllllllllllllllllllll}
10 & 0 & -10 & -20 & -30 & -40 & -50 & -60 & -70 & -80 & -90 & -100 & -110 & -120 & -130 & -140 & -150 & -160 & -170 & -180 & -190 & -200 & -210 \\
\hline
\end{tabular} 
hwu-210608-206-79dsm.10.fid

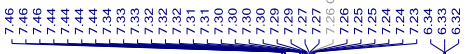<smiles>CCCOC1=CCc2ccccc21</smiles>

Wh

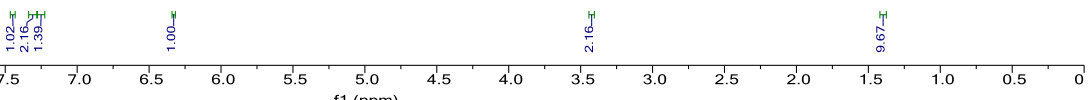

hwu-210608-206-79dsm.11.fid 0
0

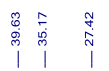

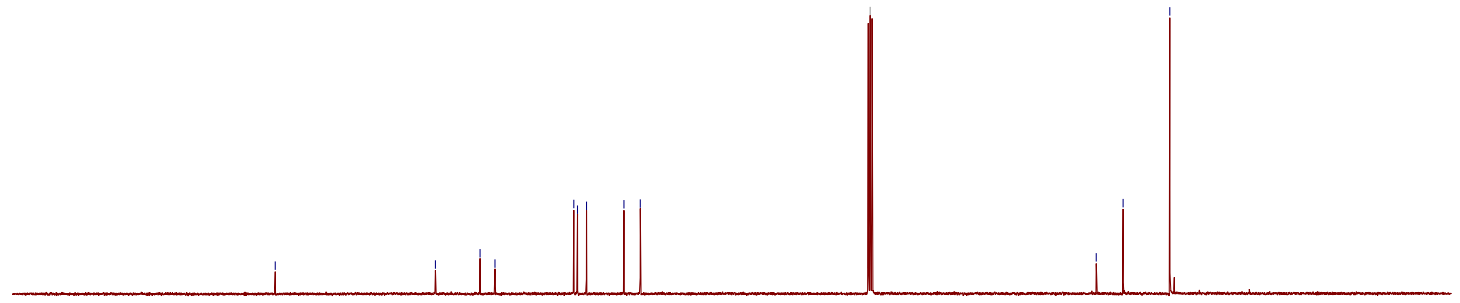

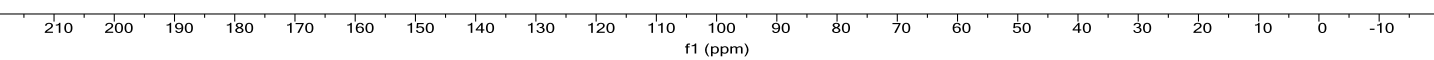




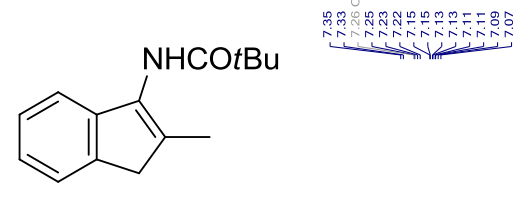

$\int / / \int S$
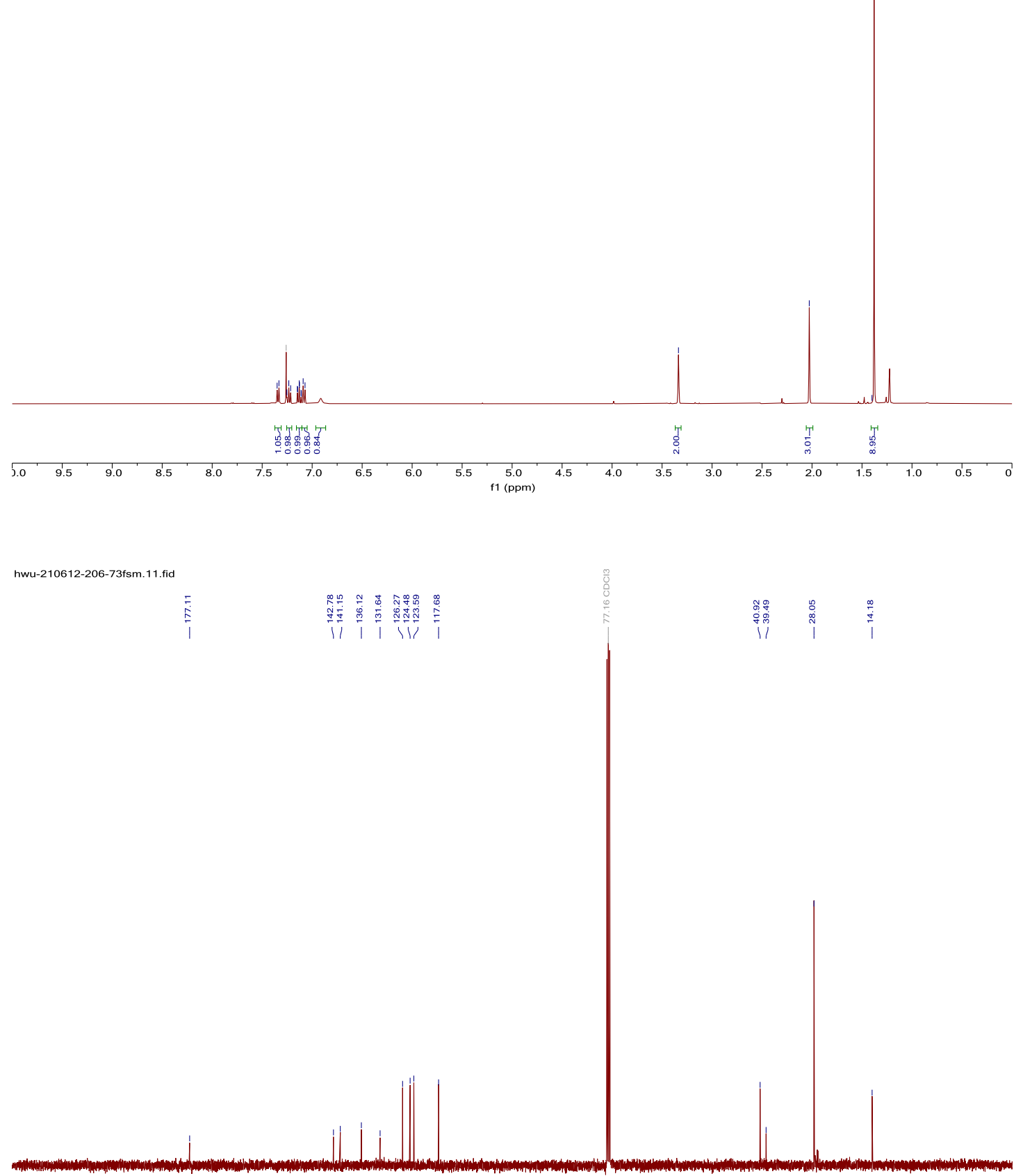

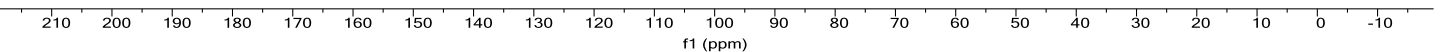


hwu-210604-206-73dsm.10.fid

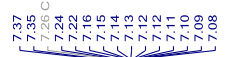

(NHCOtBu

$1111 /$

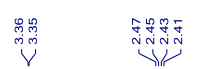

我

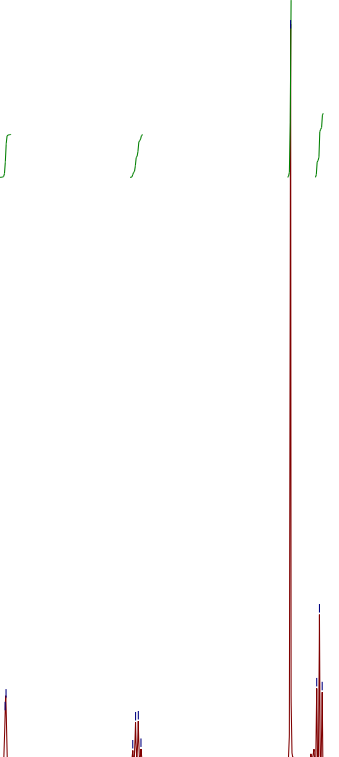

Iin

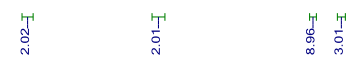

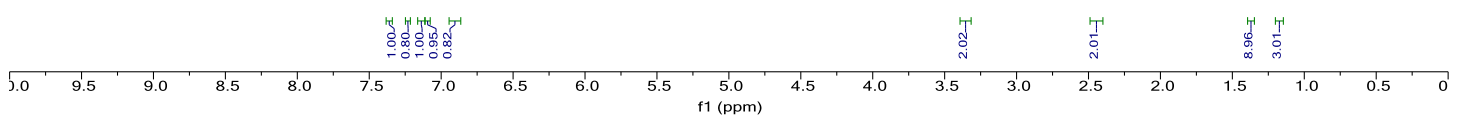

hwu-210604-206-73dsm.11.fid

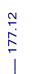

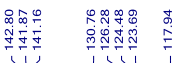

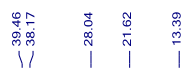

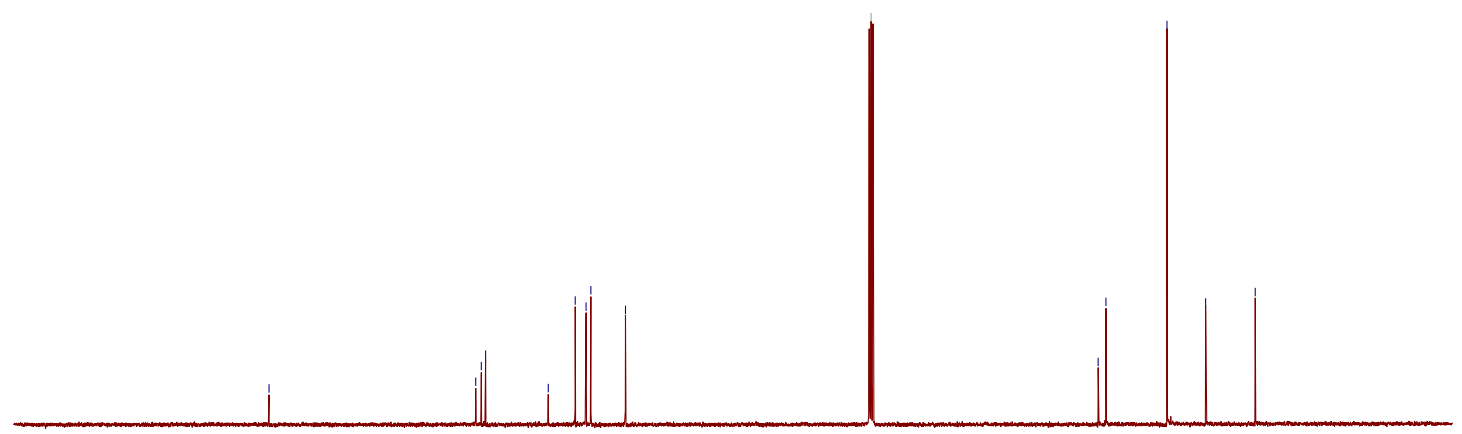

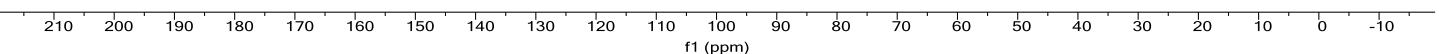


hwu-2021061506-84bsm2.1.fid
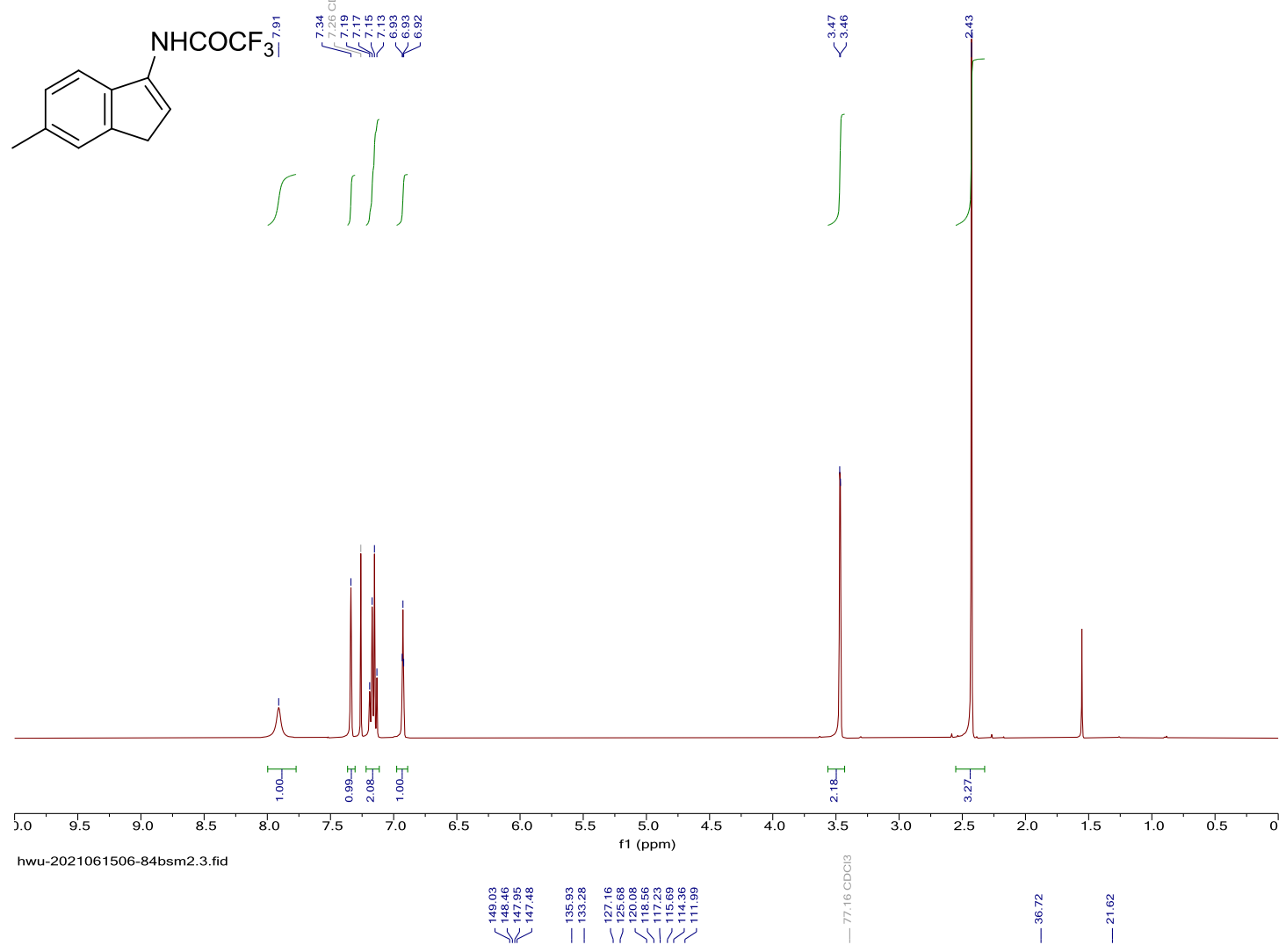

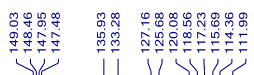

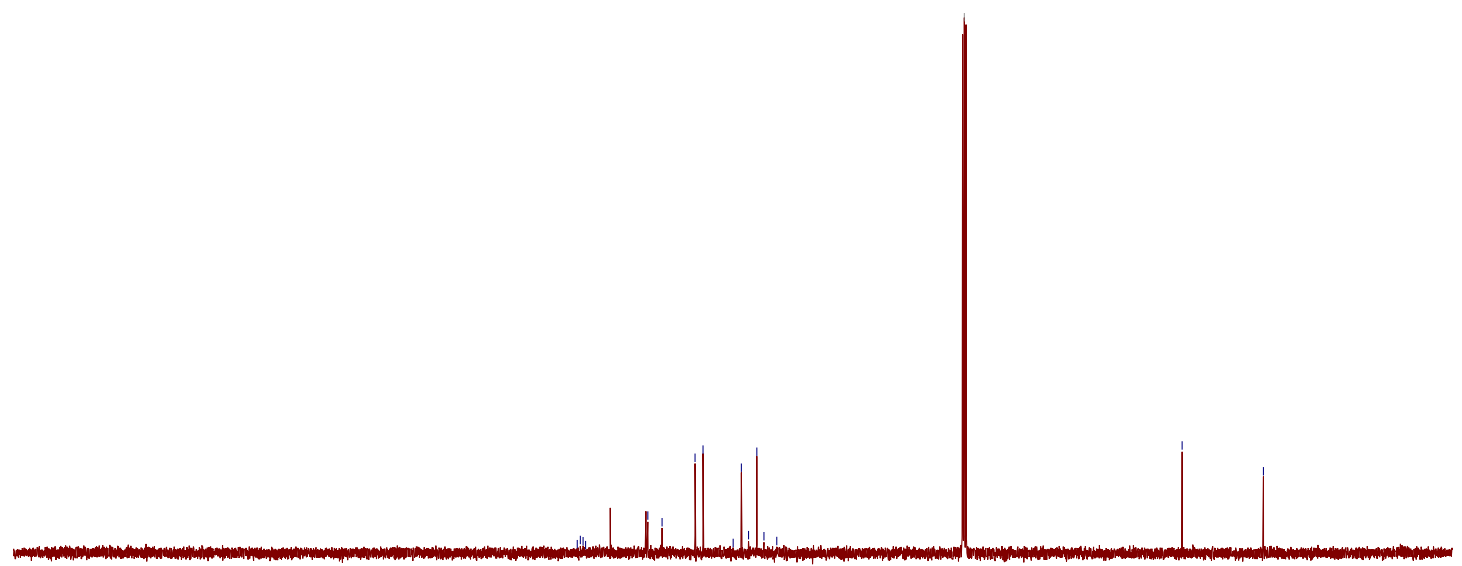

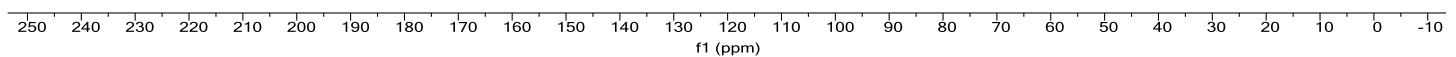




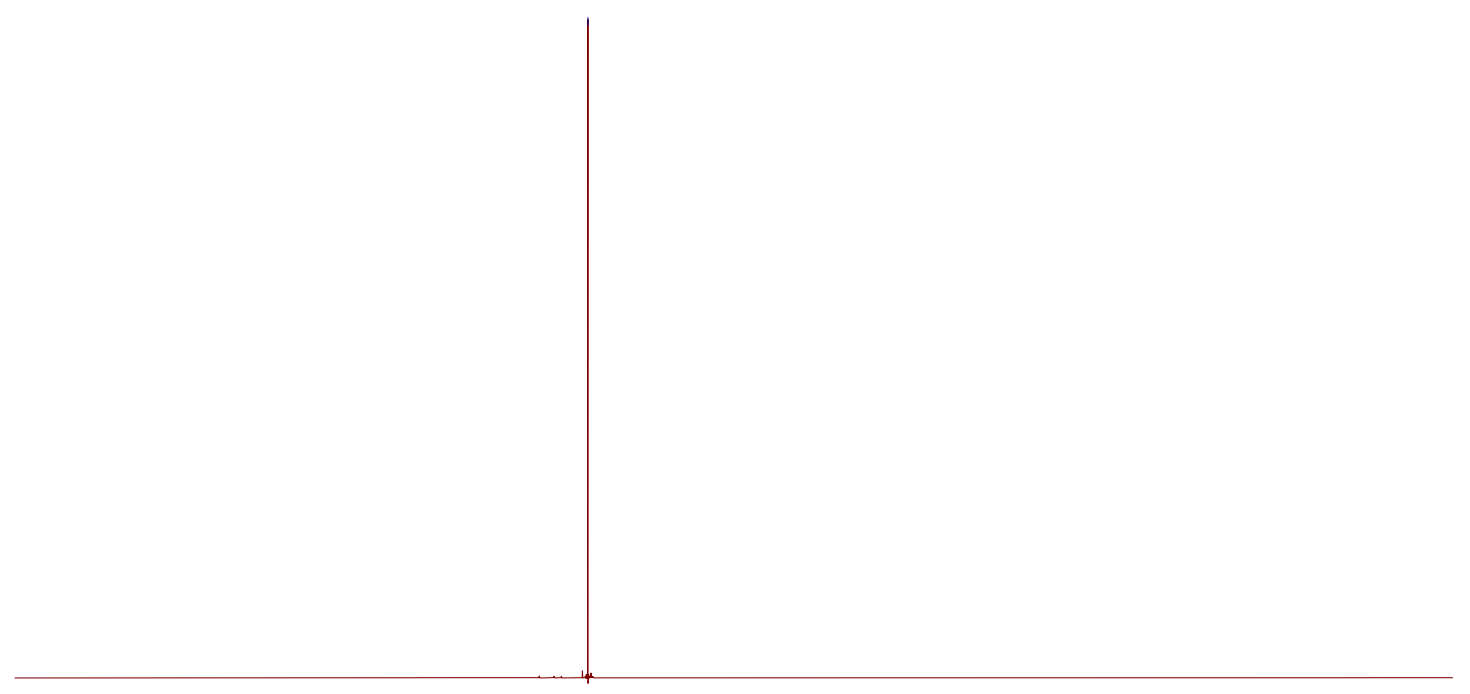

\begin{tabular}{llllllllllllllllllllllllllll}
\hline & 0 & 10 & 0 & -10 & -20 & -30 & -40 & -50 & -60 & -70 & -80 & -90 & -100 & -110 & -120 & -130 & -140 & -150 & -160 & -170 & -180 & -190 & -200 & -210 & -2
\end{tabular} 
hwu-210612-206-79bsm.10.fid
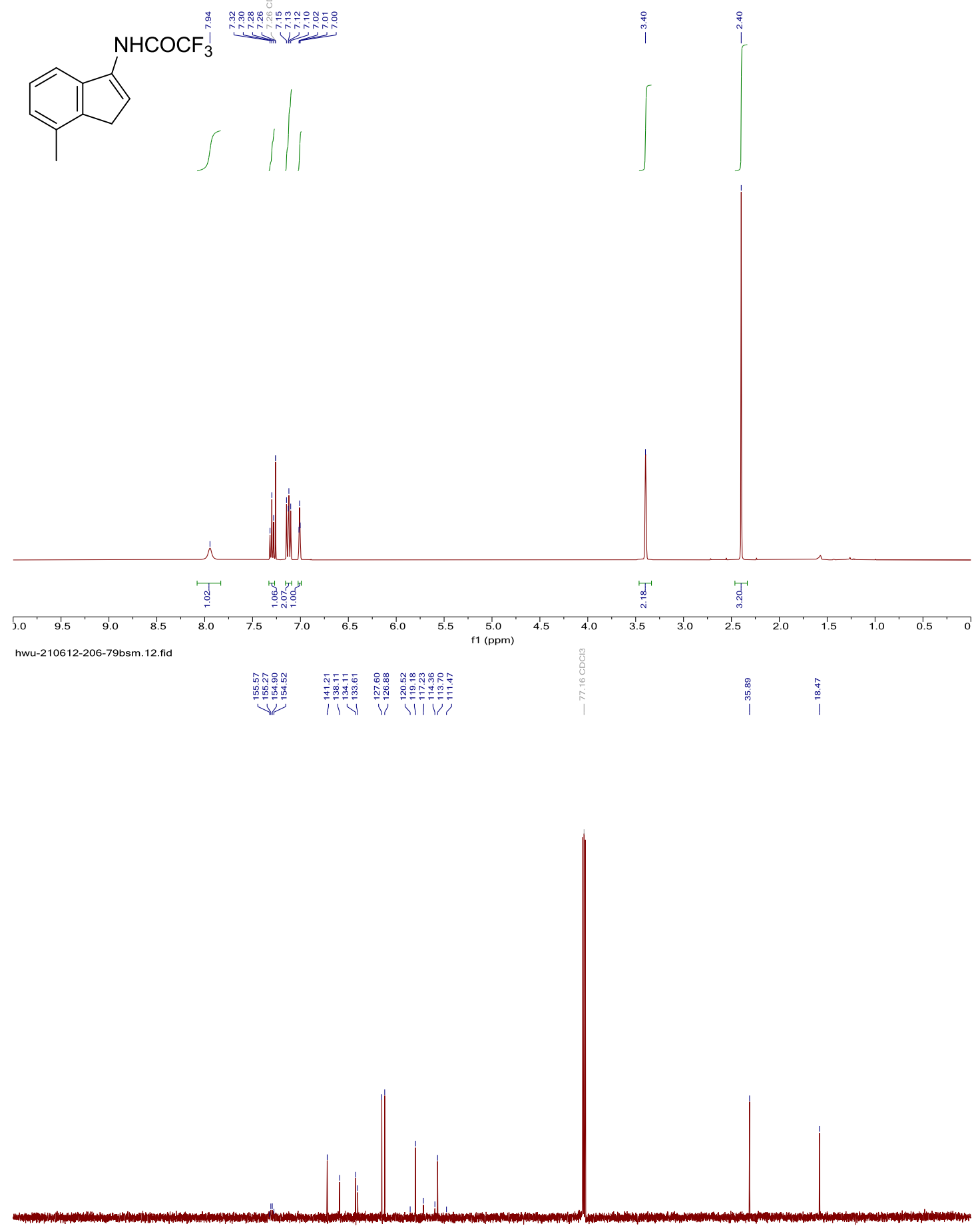

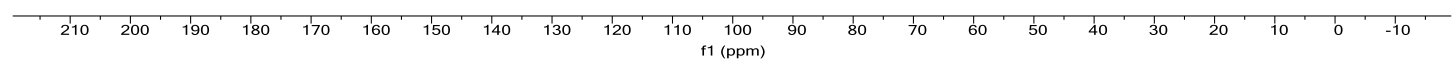




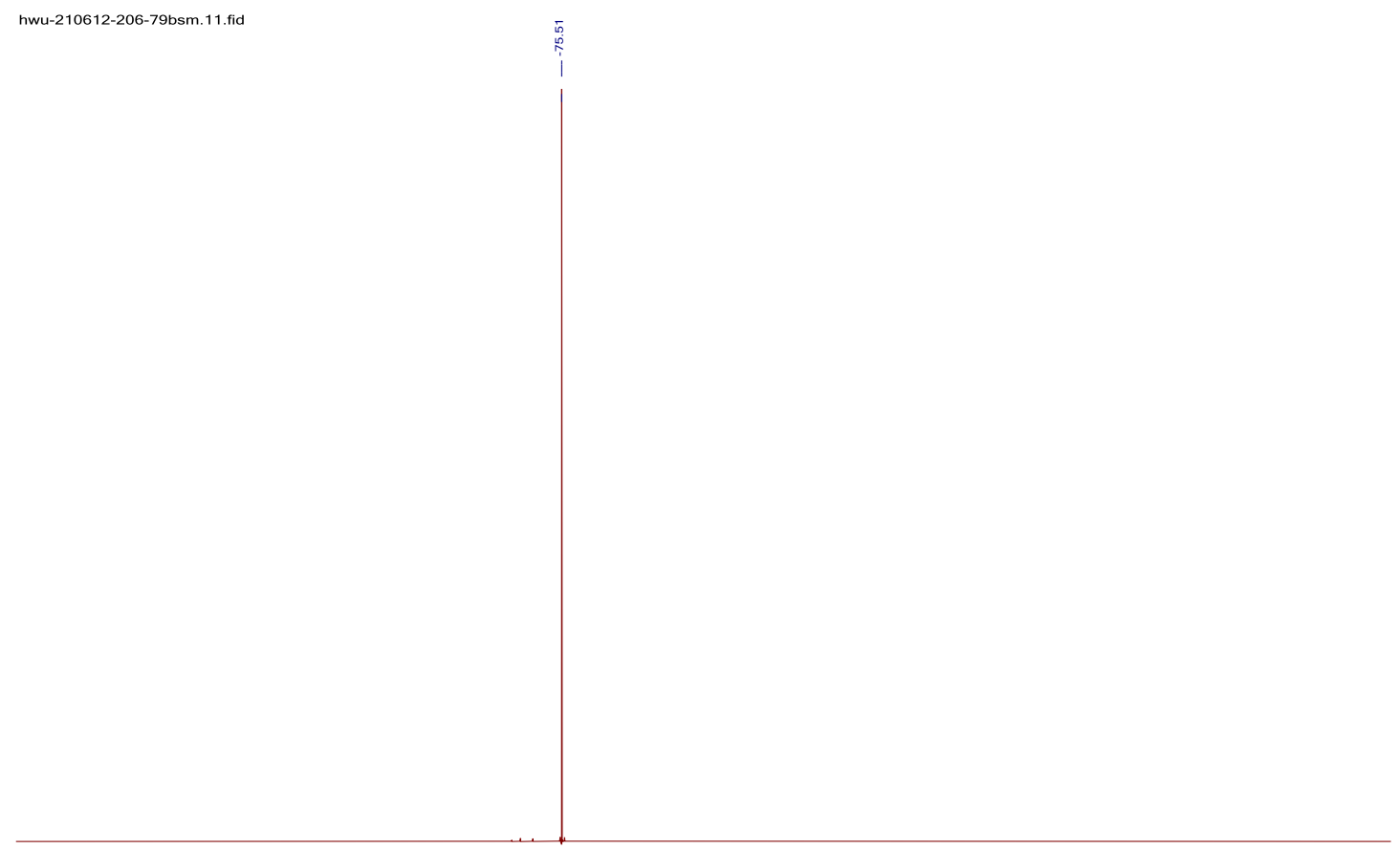

$\begin{array}{lllllllllllllllllllllll}10 & 0 & -10 & -20 & -30 & -40 & -50 & -60 & -70 & -80 & -90 & -100 & -110 & -120 & -130 & -140 & -150 & -160 & -170 & -180 & -190 & -200 & -210\end{array}$ 

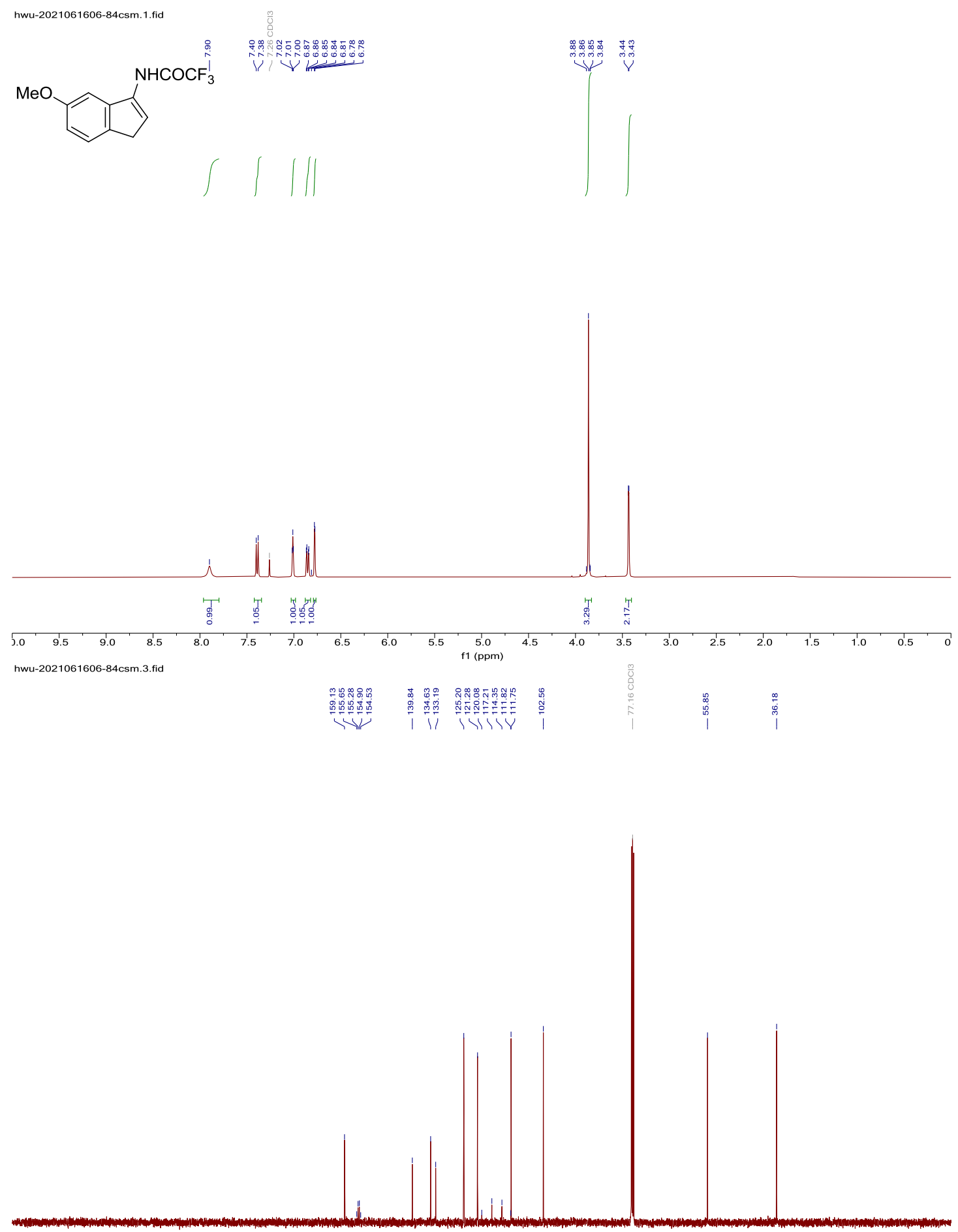

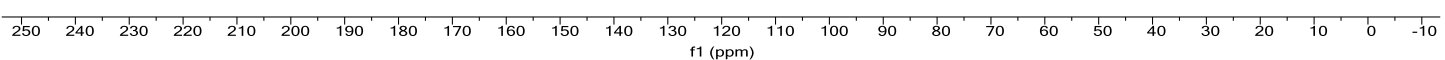




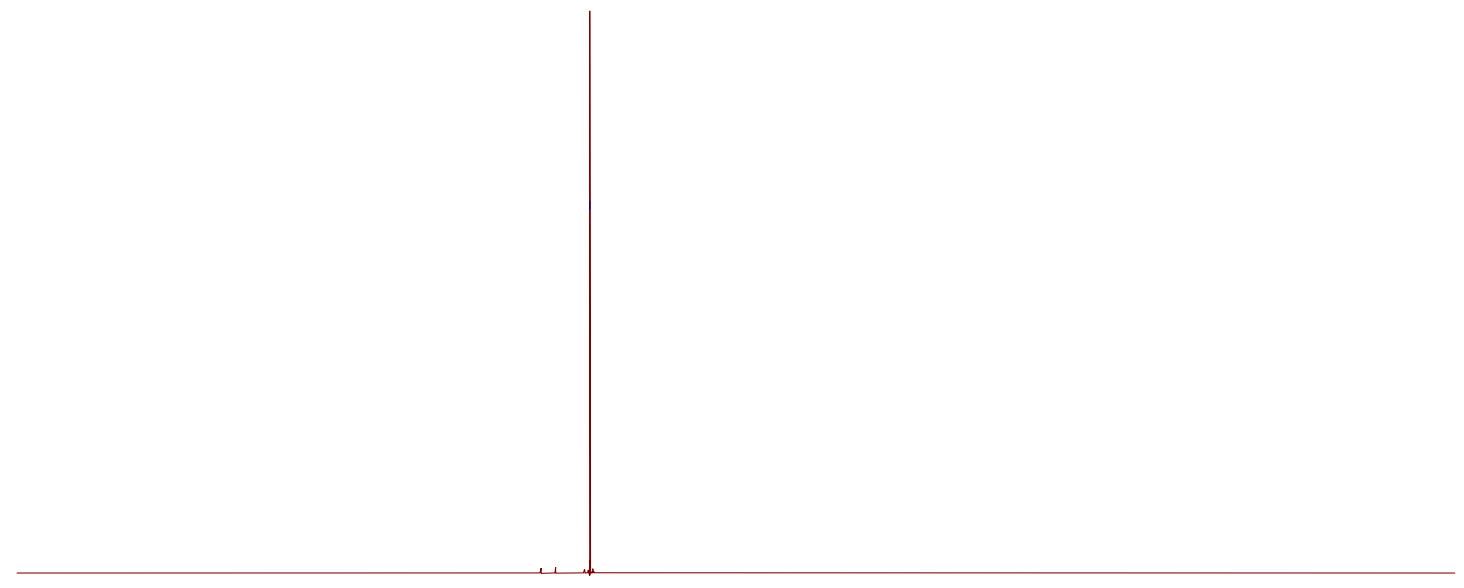

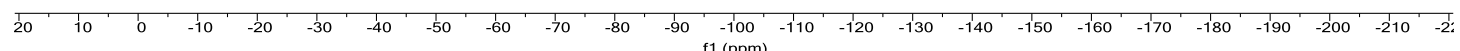



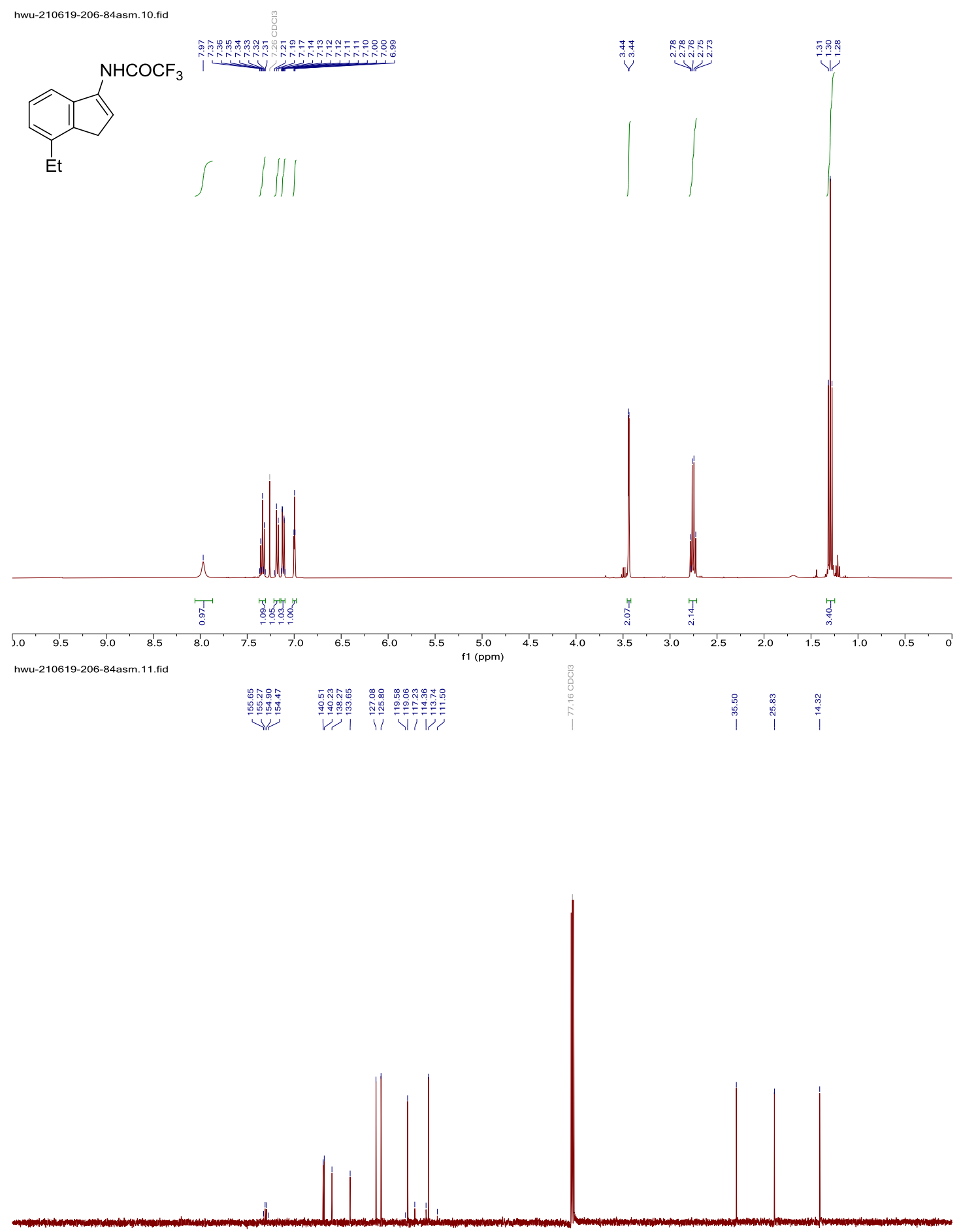

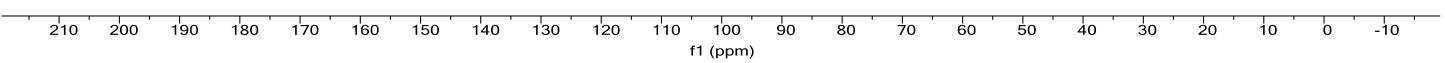




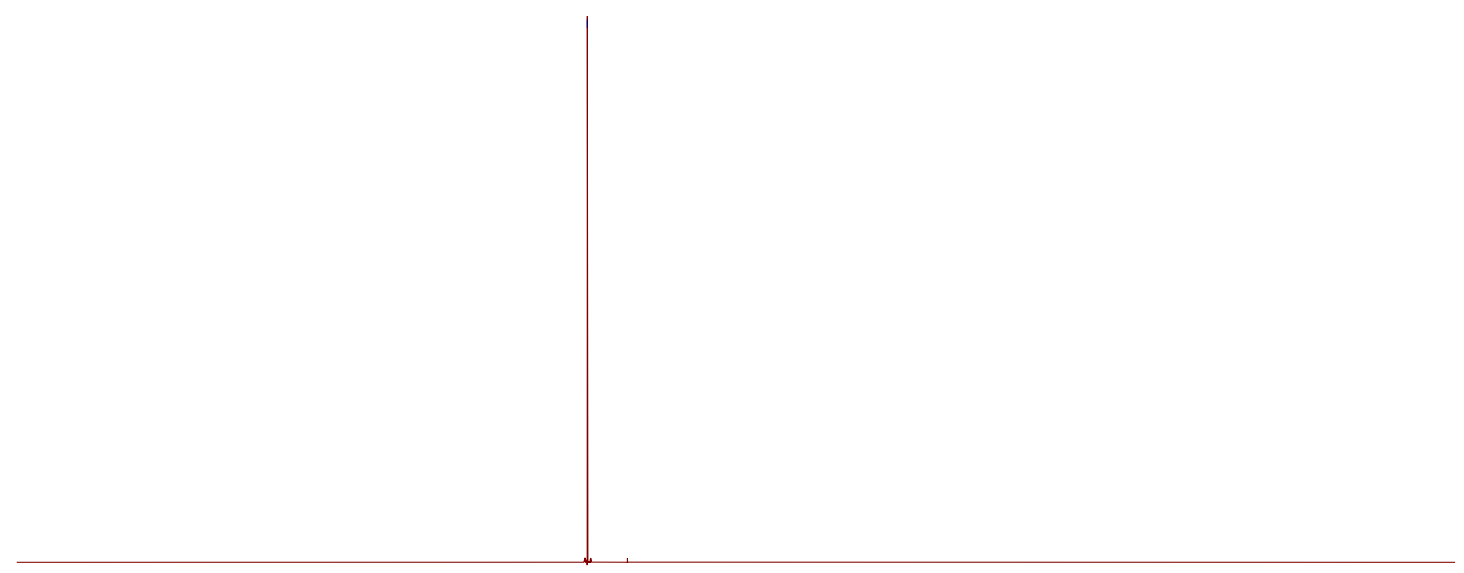

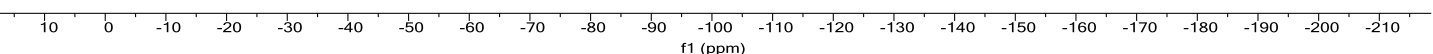


yanji-20210612-9-sm-9-DMSO.1.fid
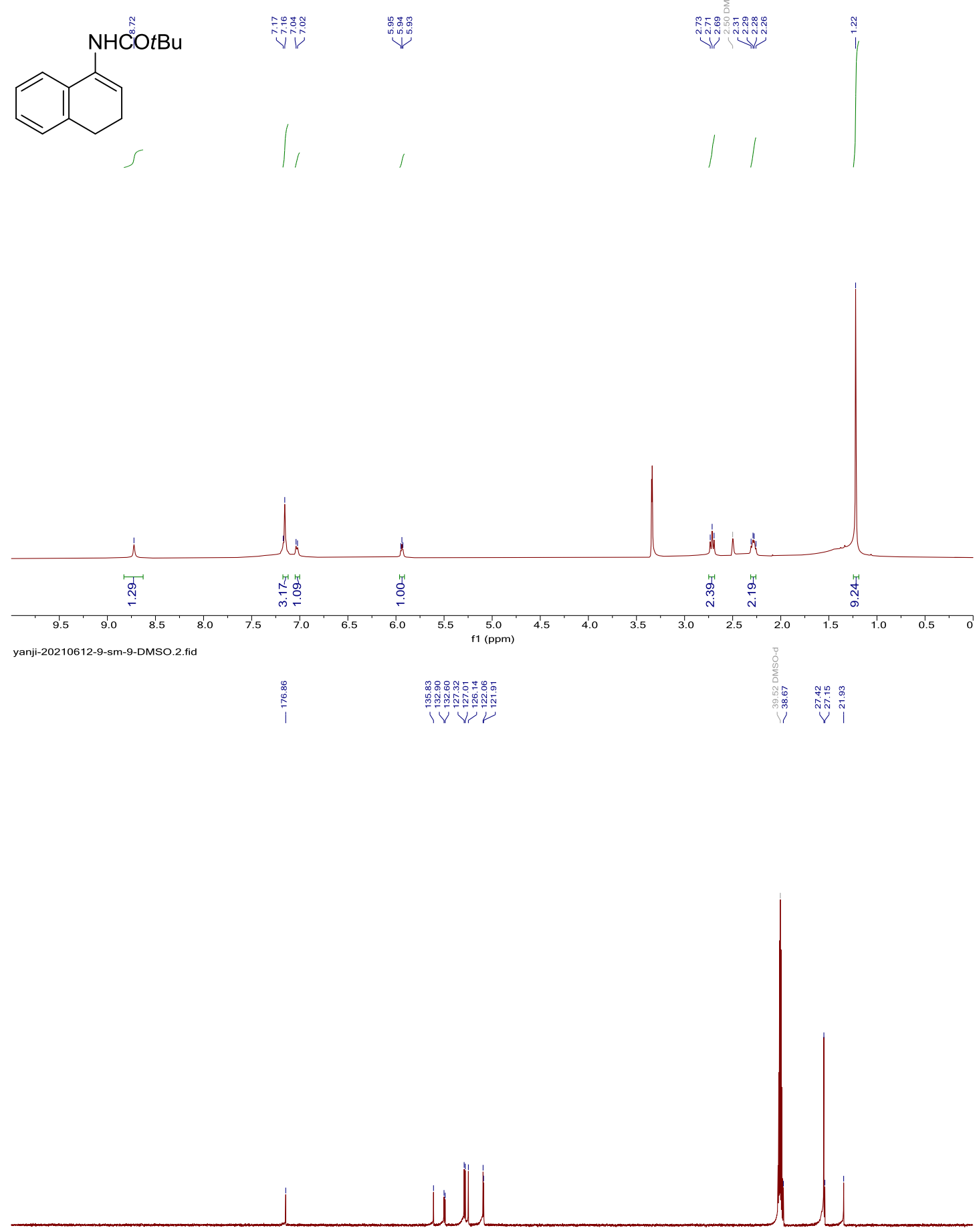

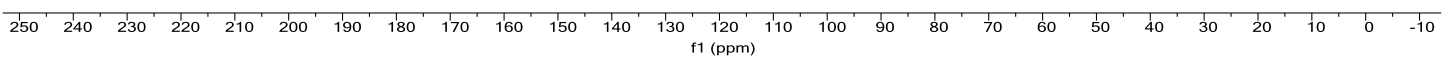



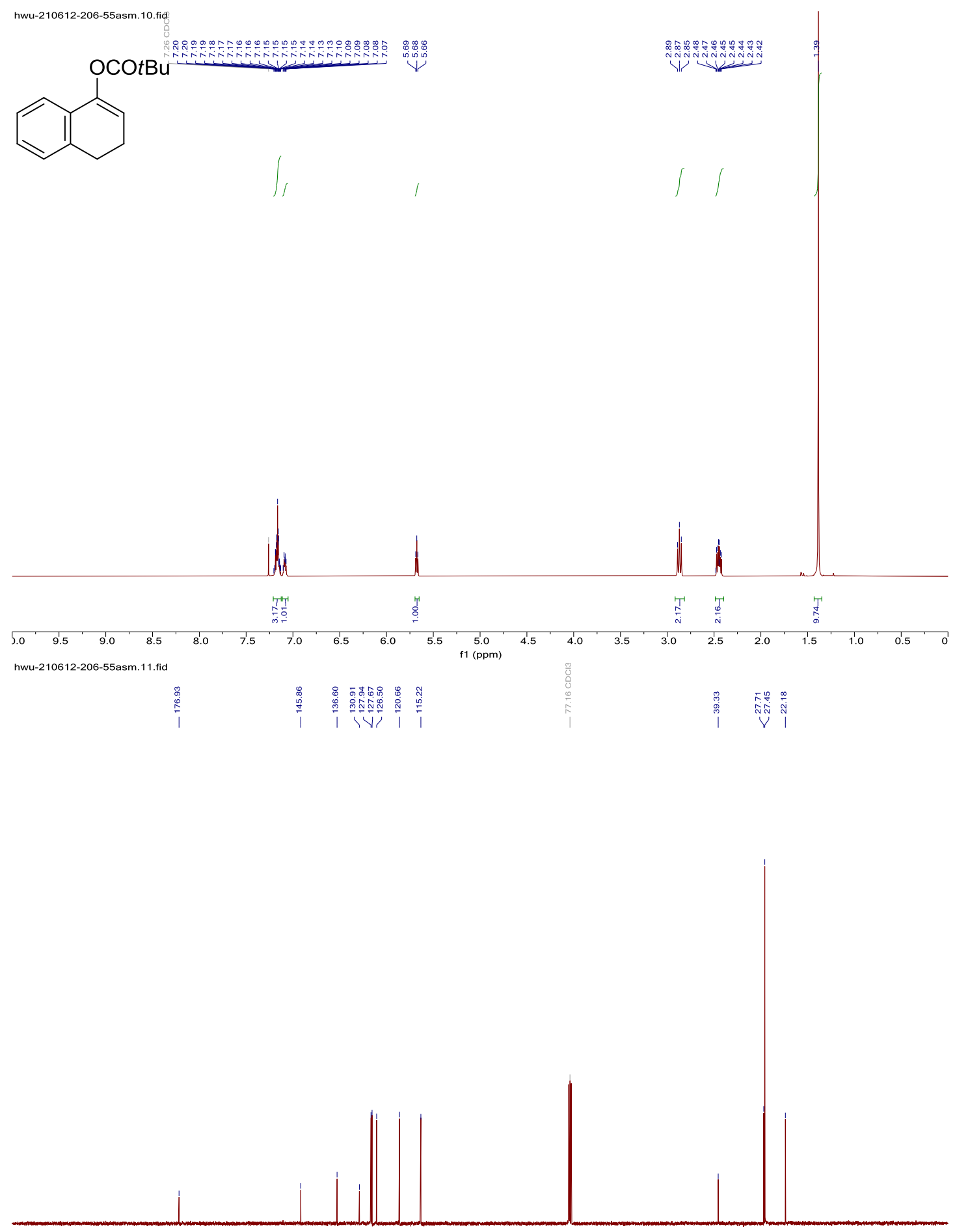

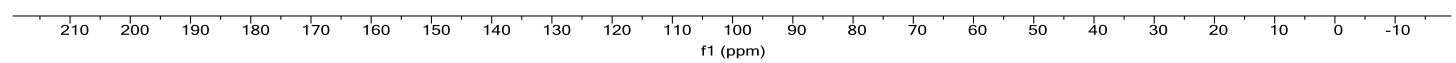


hwu-210704-206-88dsing. 10.fid

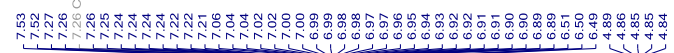
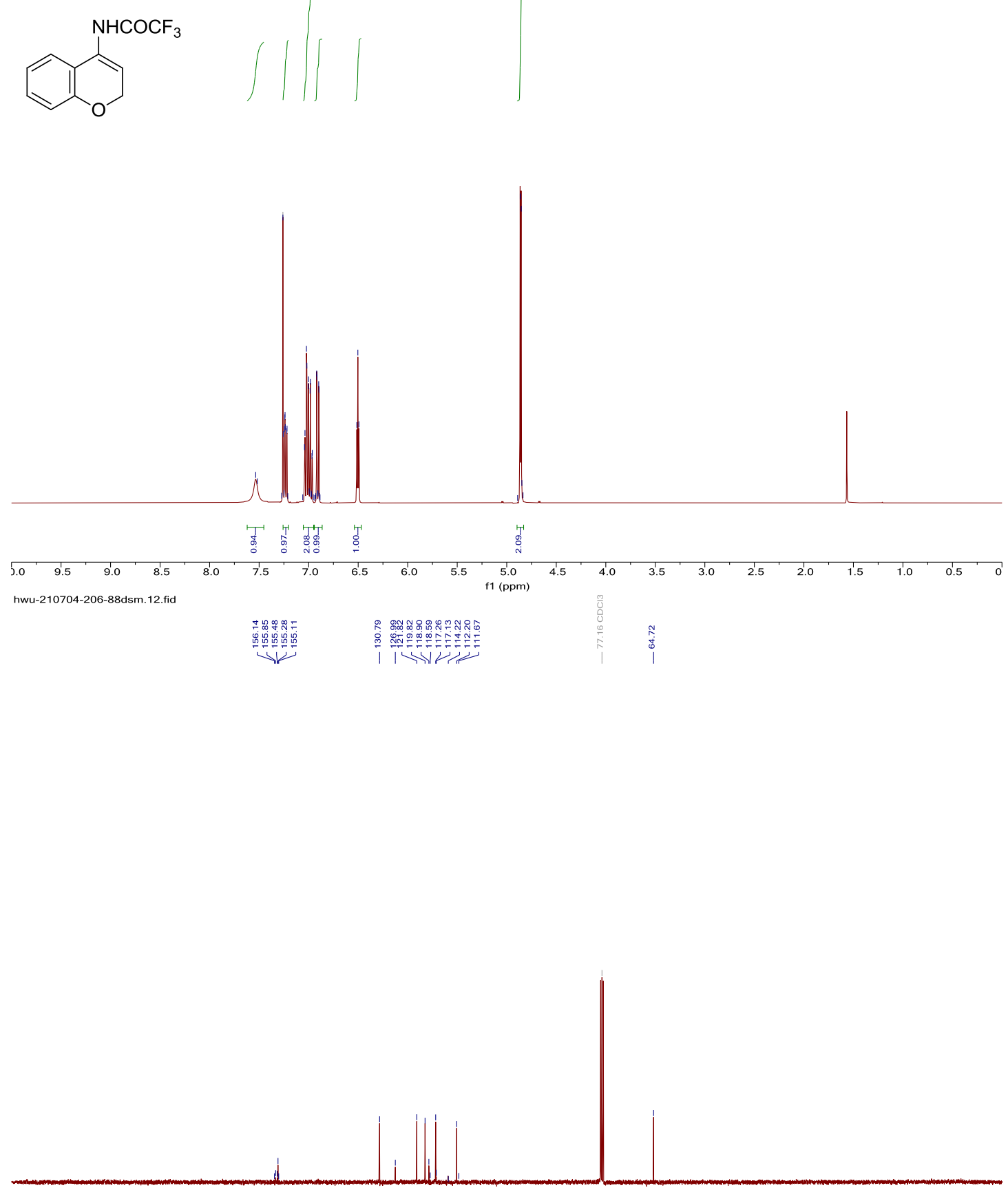

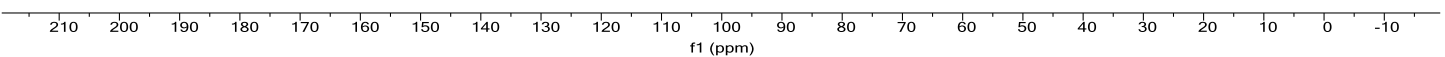




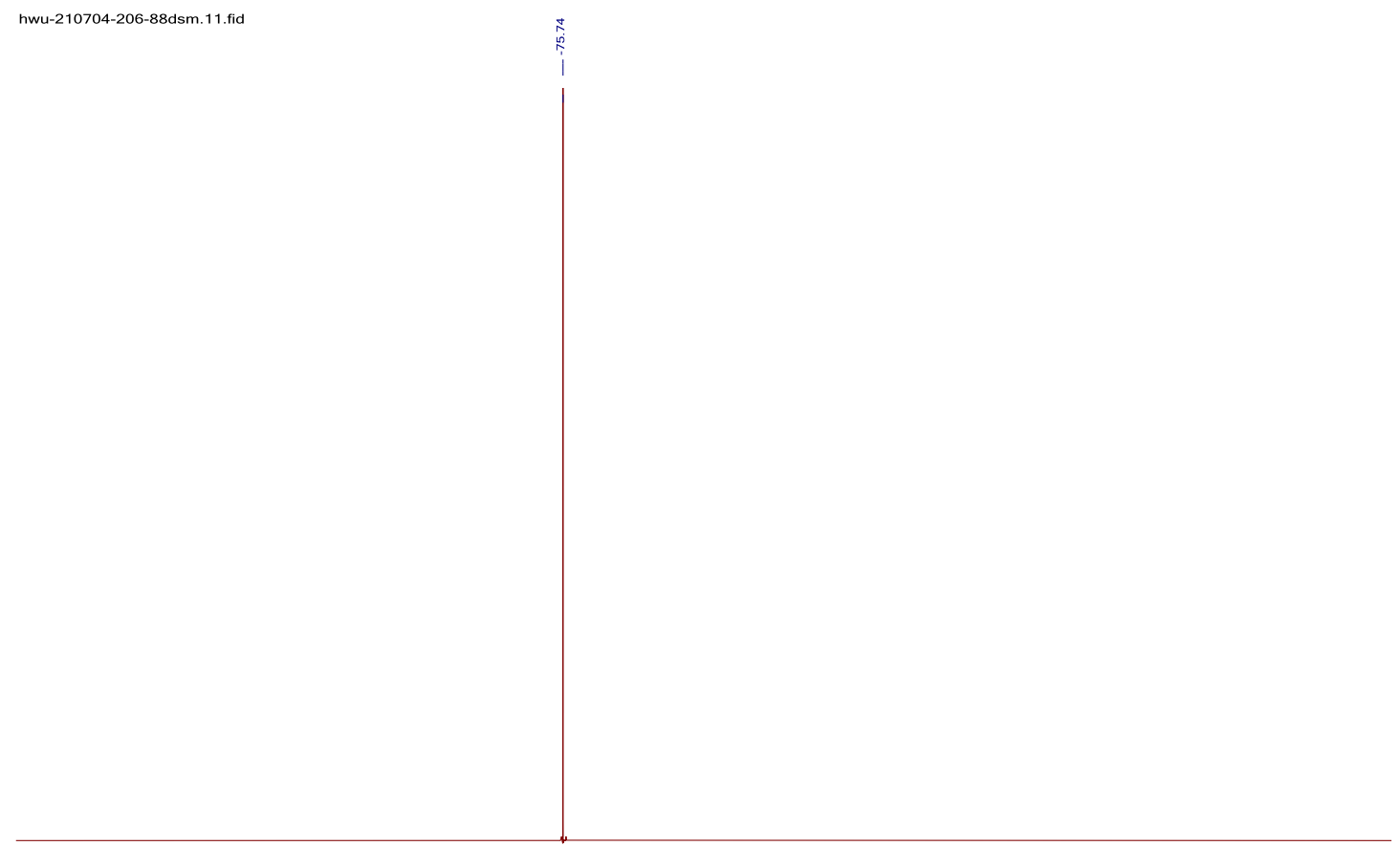

\begin{tabular}{lllllllllllllllllllllll}
10 & 0 & -10 & -20 & -30 & -40 & -50 & -60 & -70 & -80 & -90 & -100 & -110 & -120 & -130 & -140 & -150 & -160 & -170 & -180 & -190 & -100 & -1210 \\
\hline
\end{tabular} 

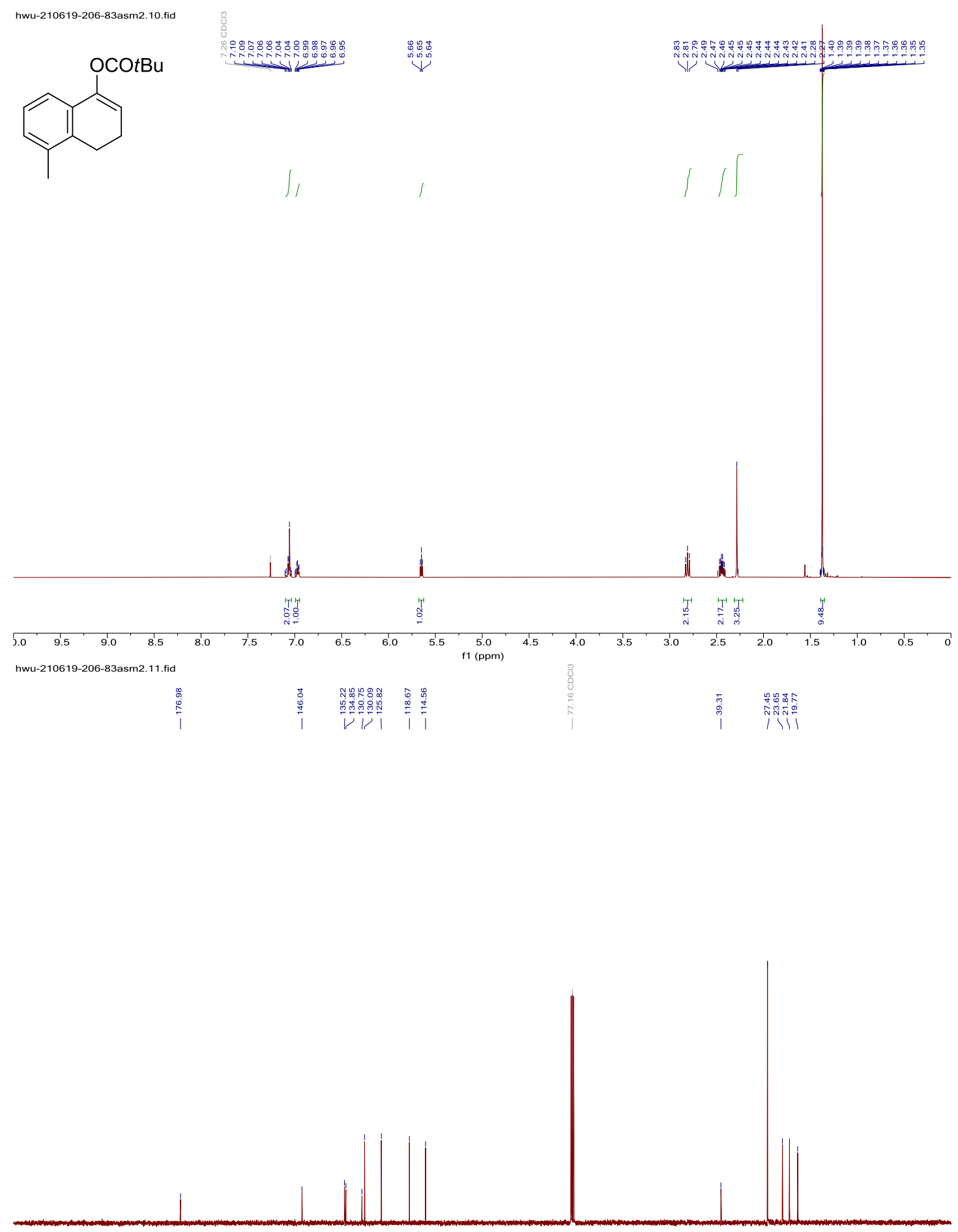

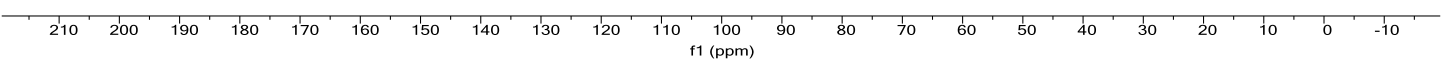




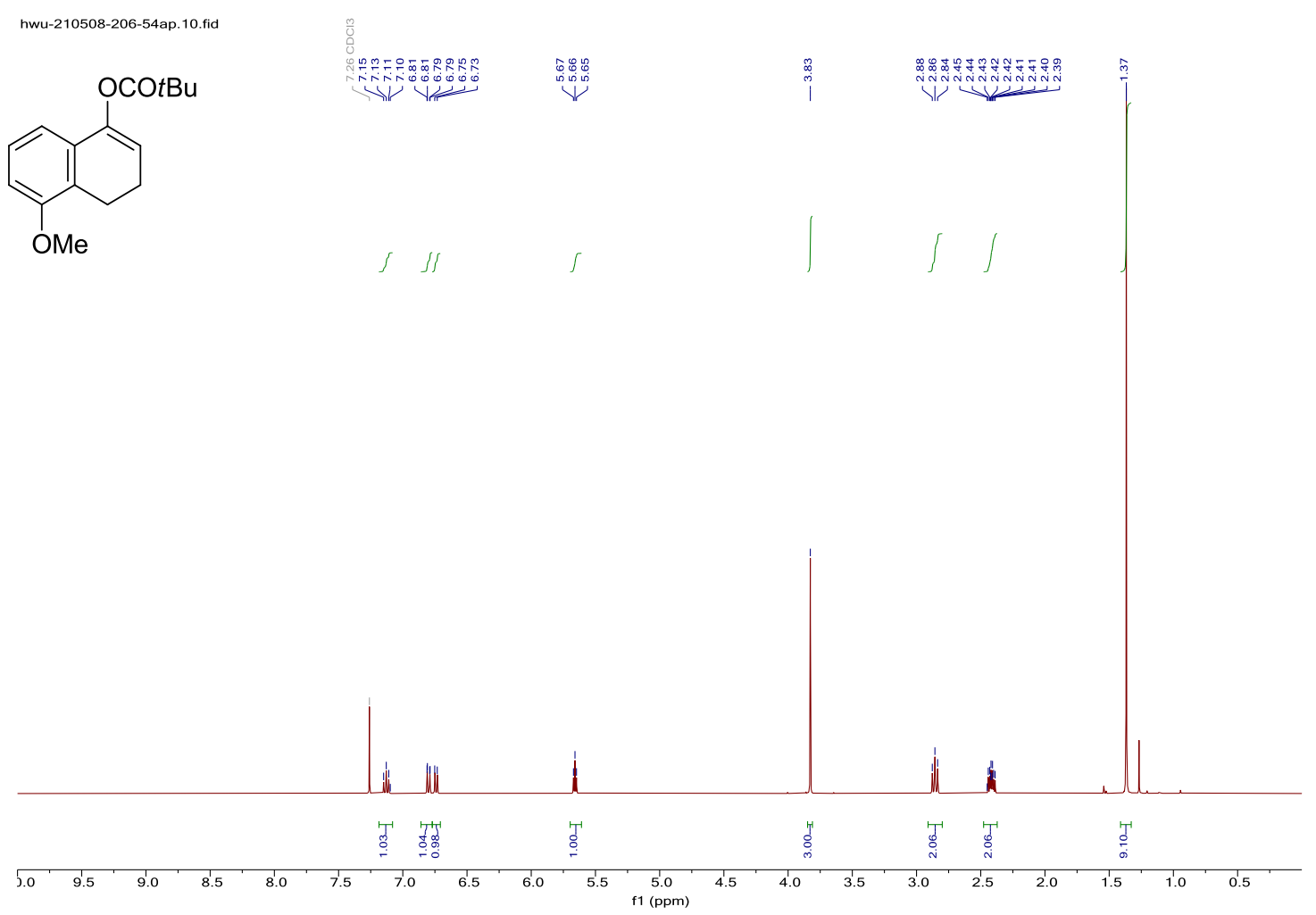

hwu-210508-206-54ap.11.fid

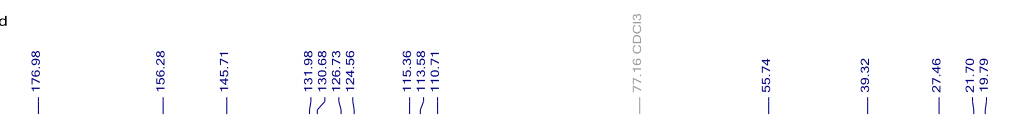

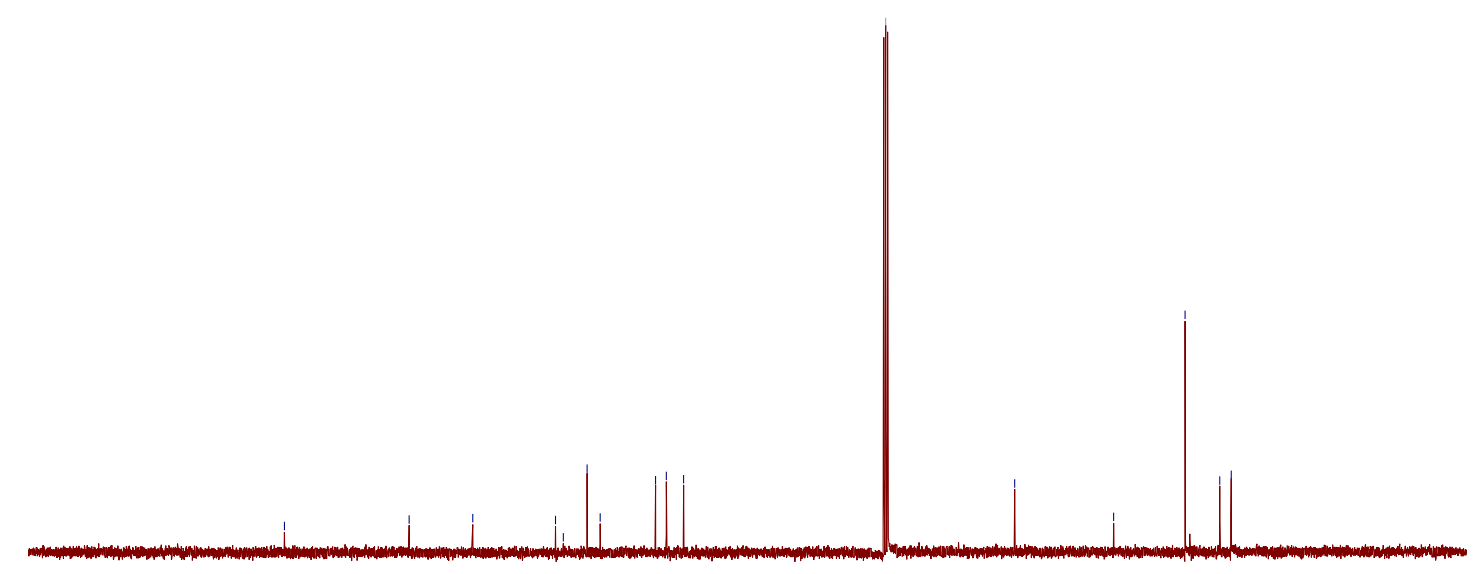




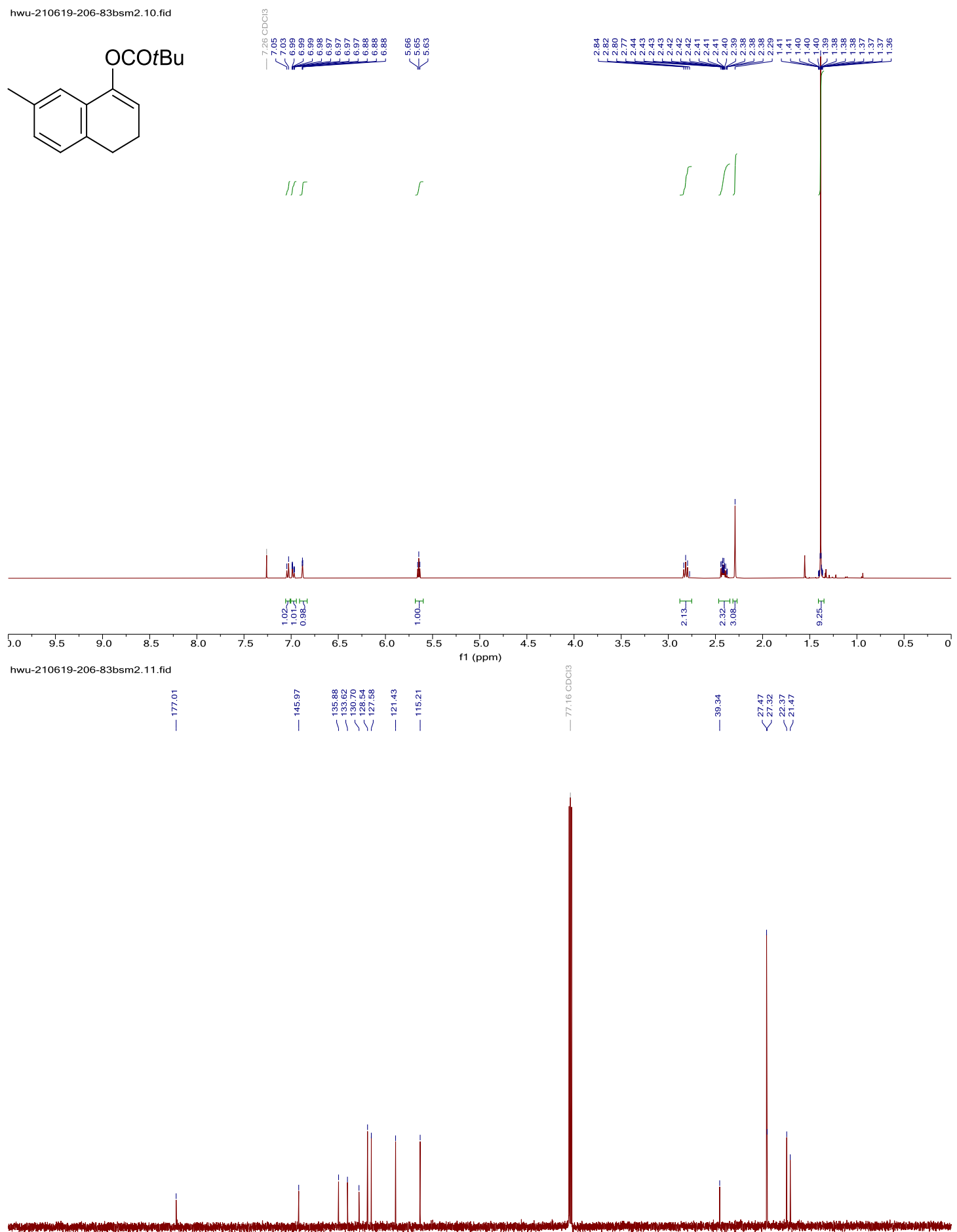

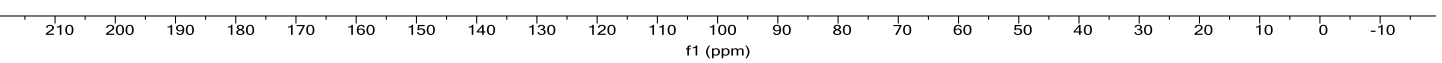




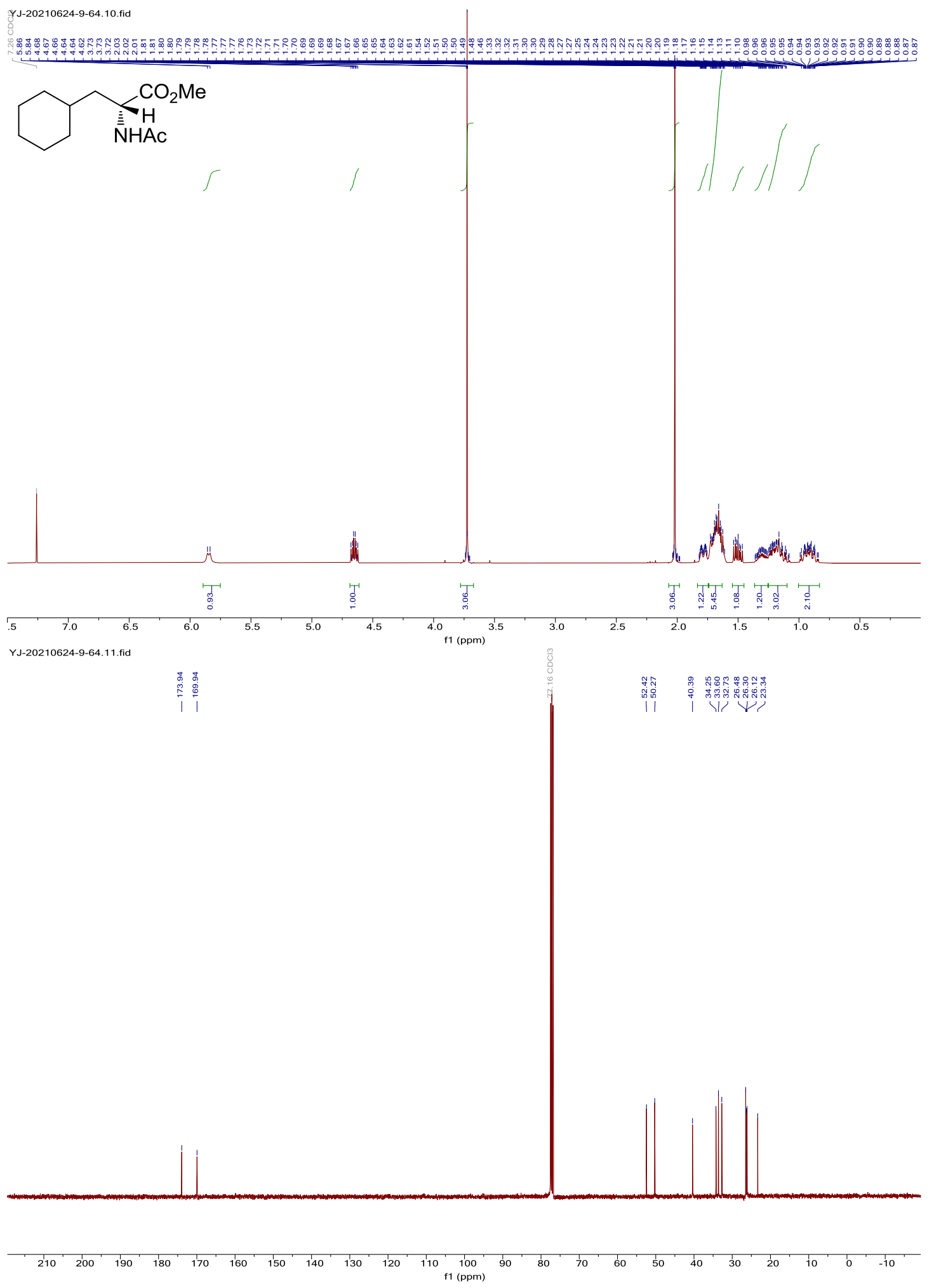

S65 

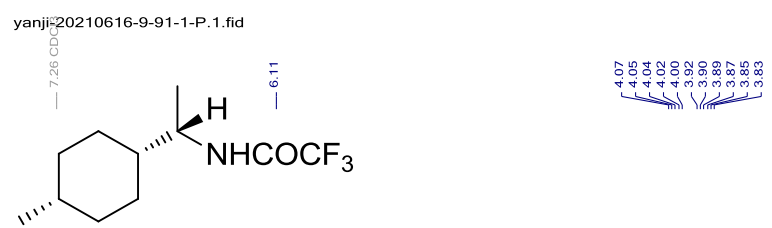

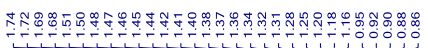

$\mathrm{NHCOCF}_{3}$

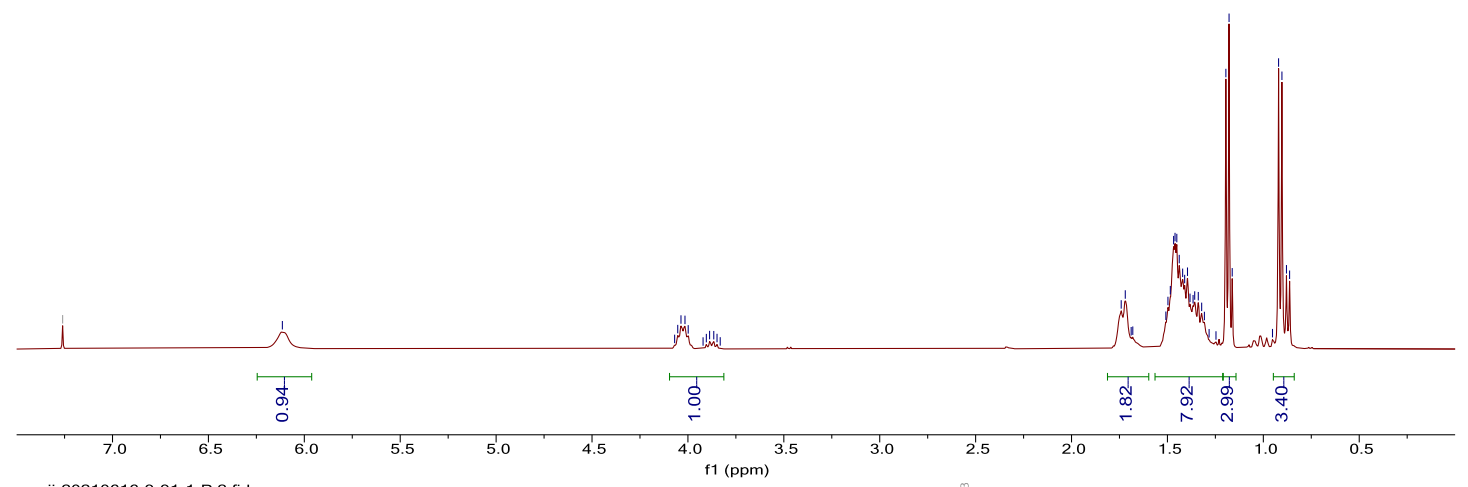
yanji-20210616-9-91-1-P.2.fid

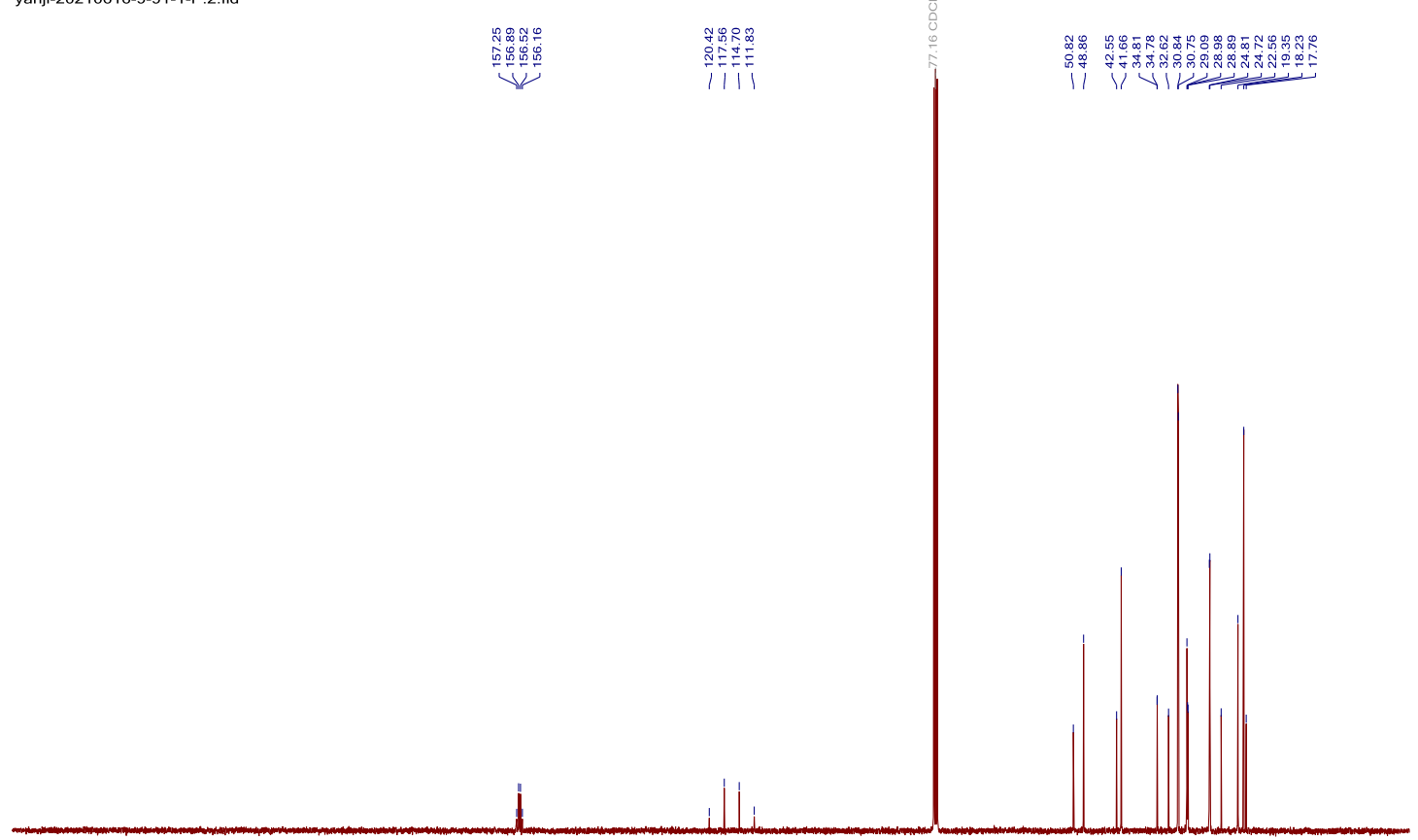

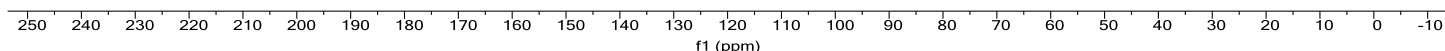




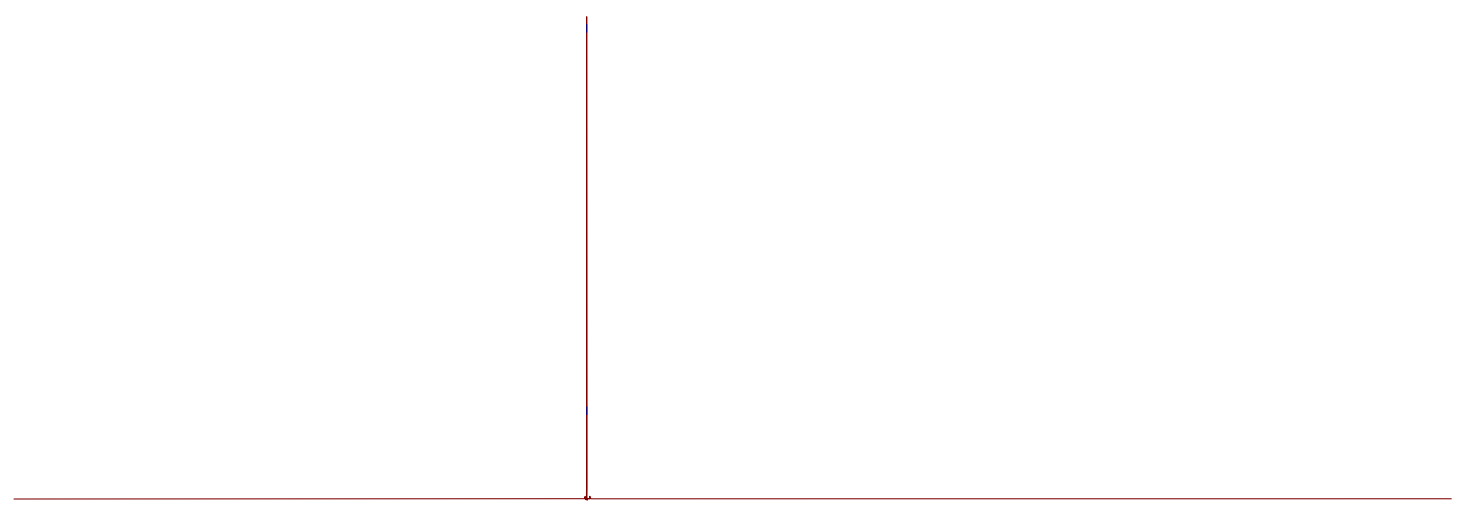

$\begin{array}{lllllllllllllllllllllllllllll}10 & 0 & -10 & -20 & -30 & -40 & -50 & -60 & -70 & -80 & -90 & -100 & -110 & -120 & -130 & -140 & -150 & -160 & -170 & -180 & -190 & -200 & -210 & 1\end{array}$ 


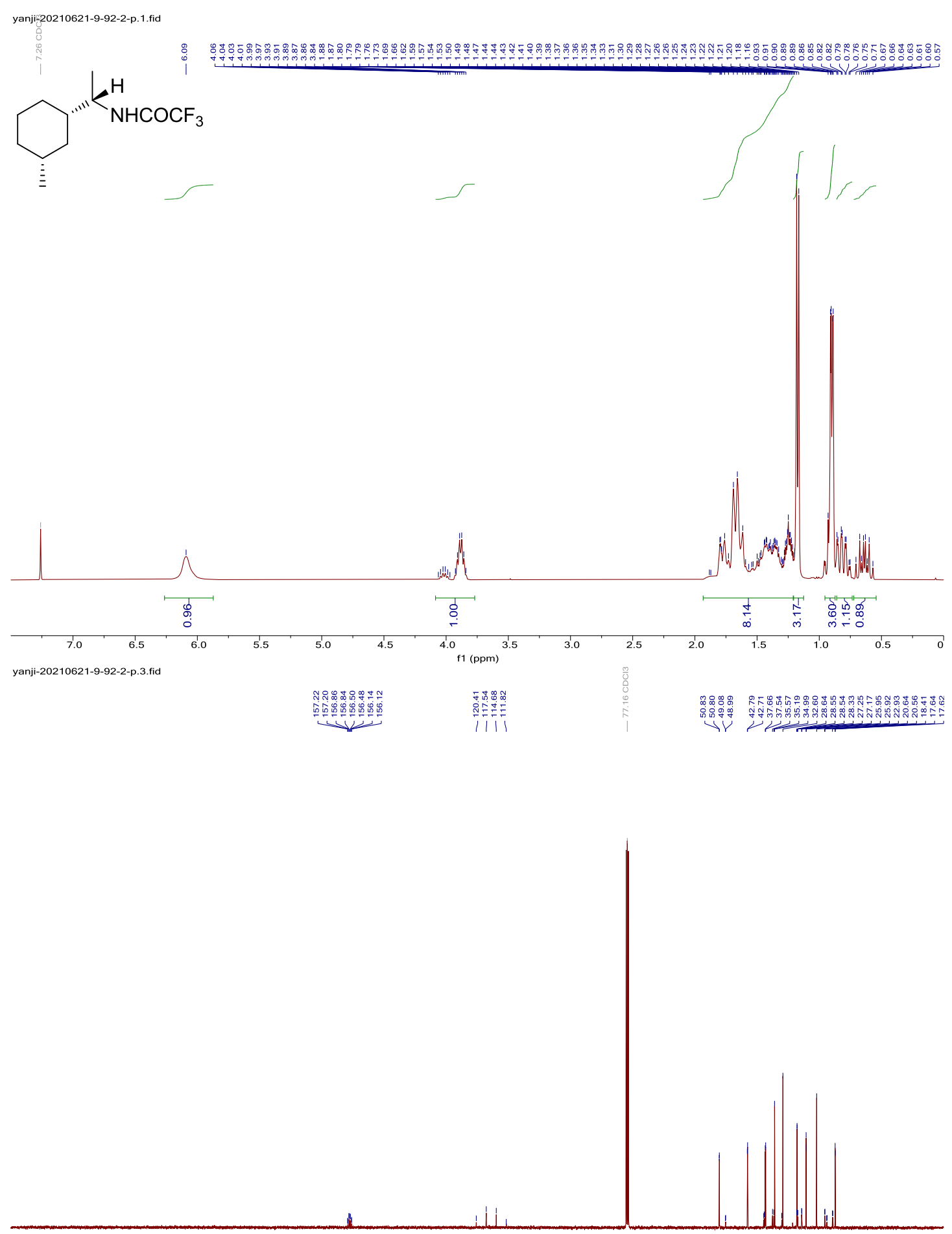

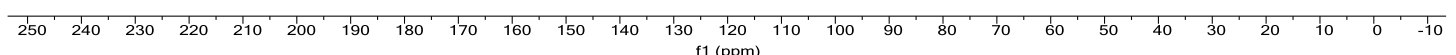




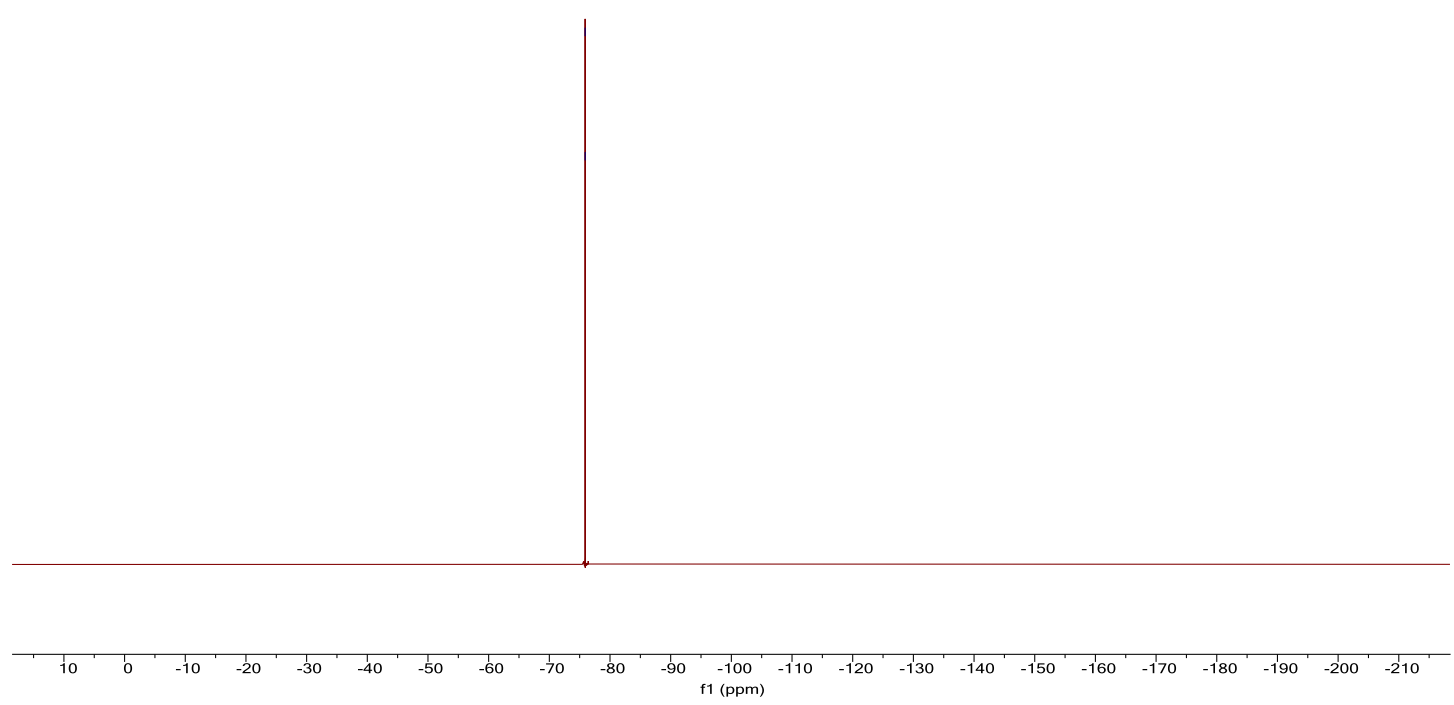




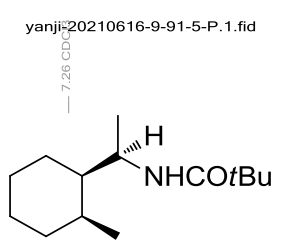

9.00\%

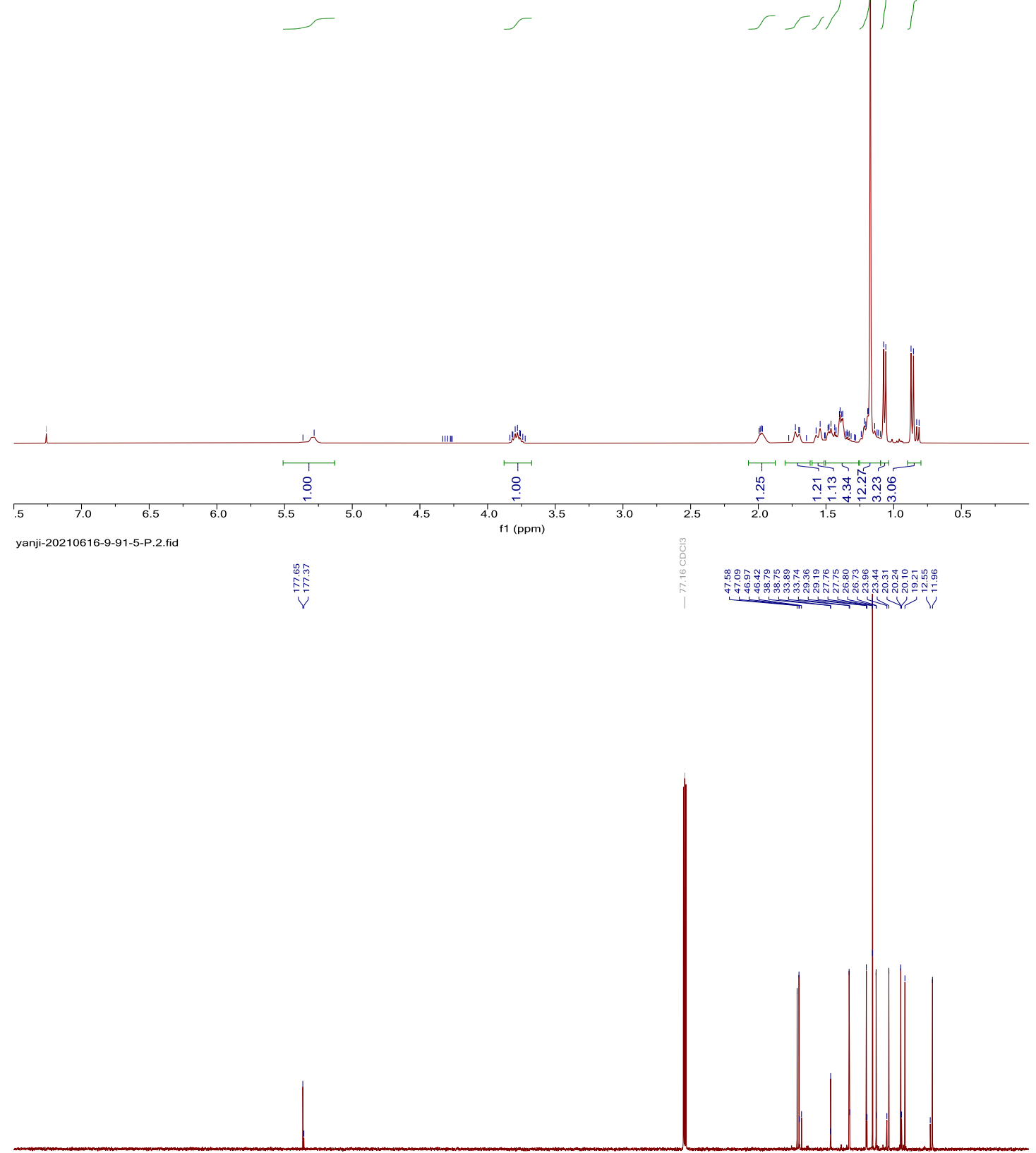

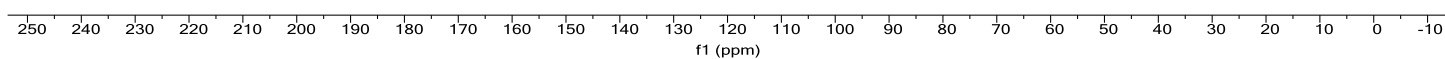




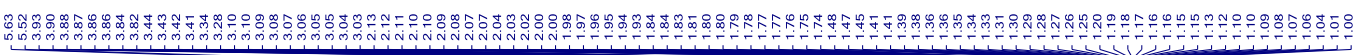
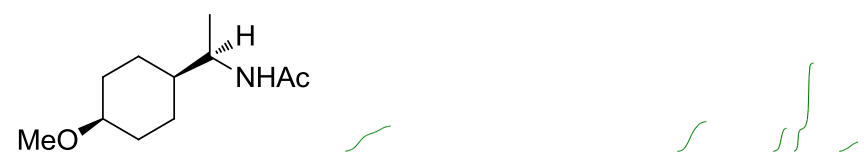

$\int$
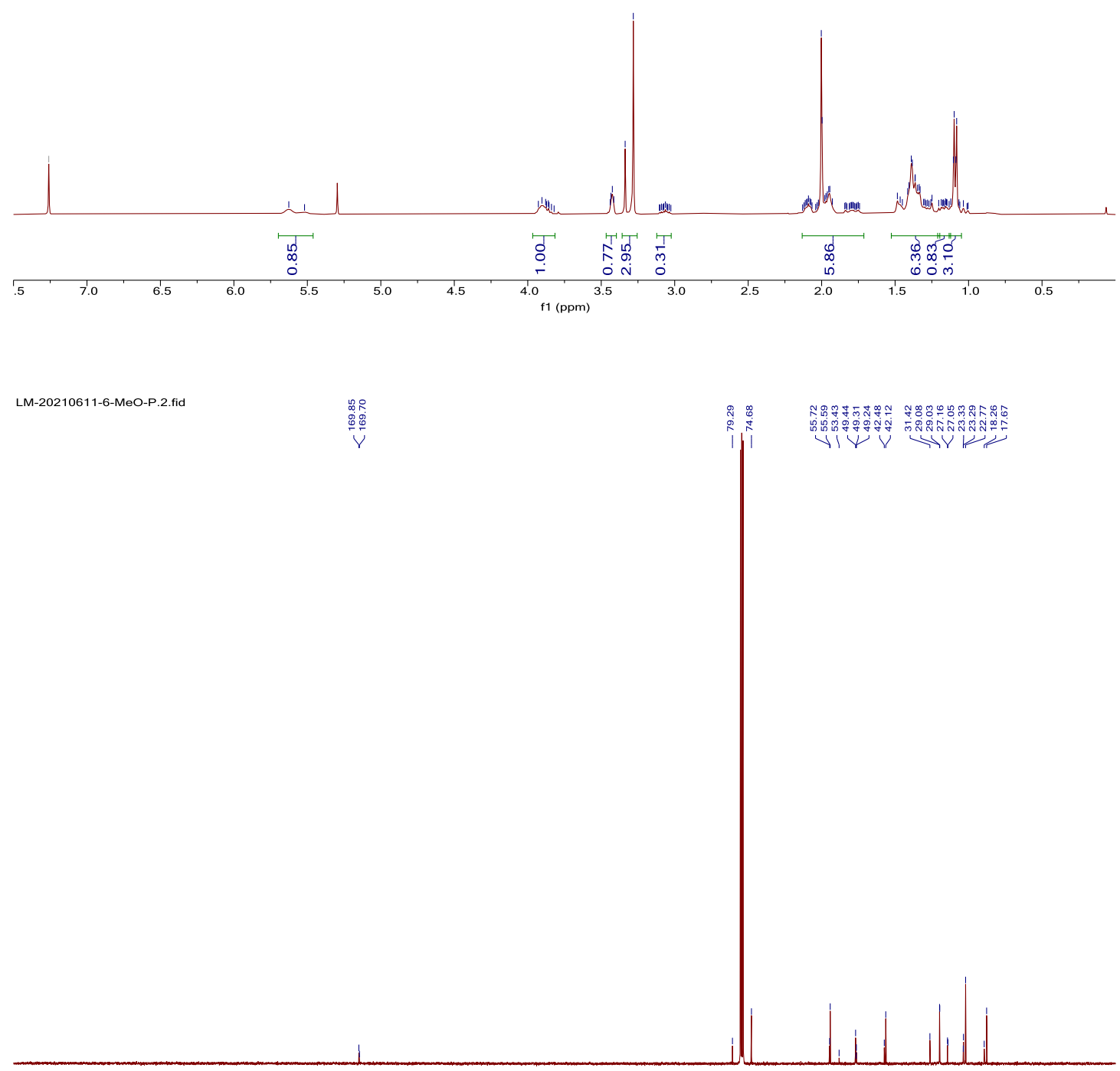

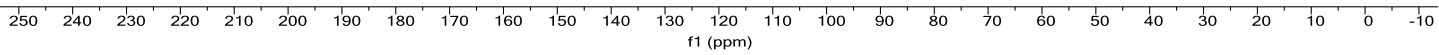

S71 

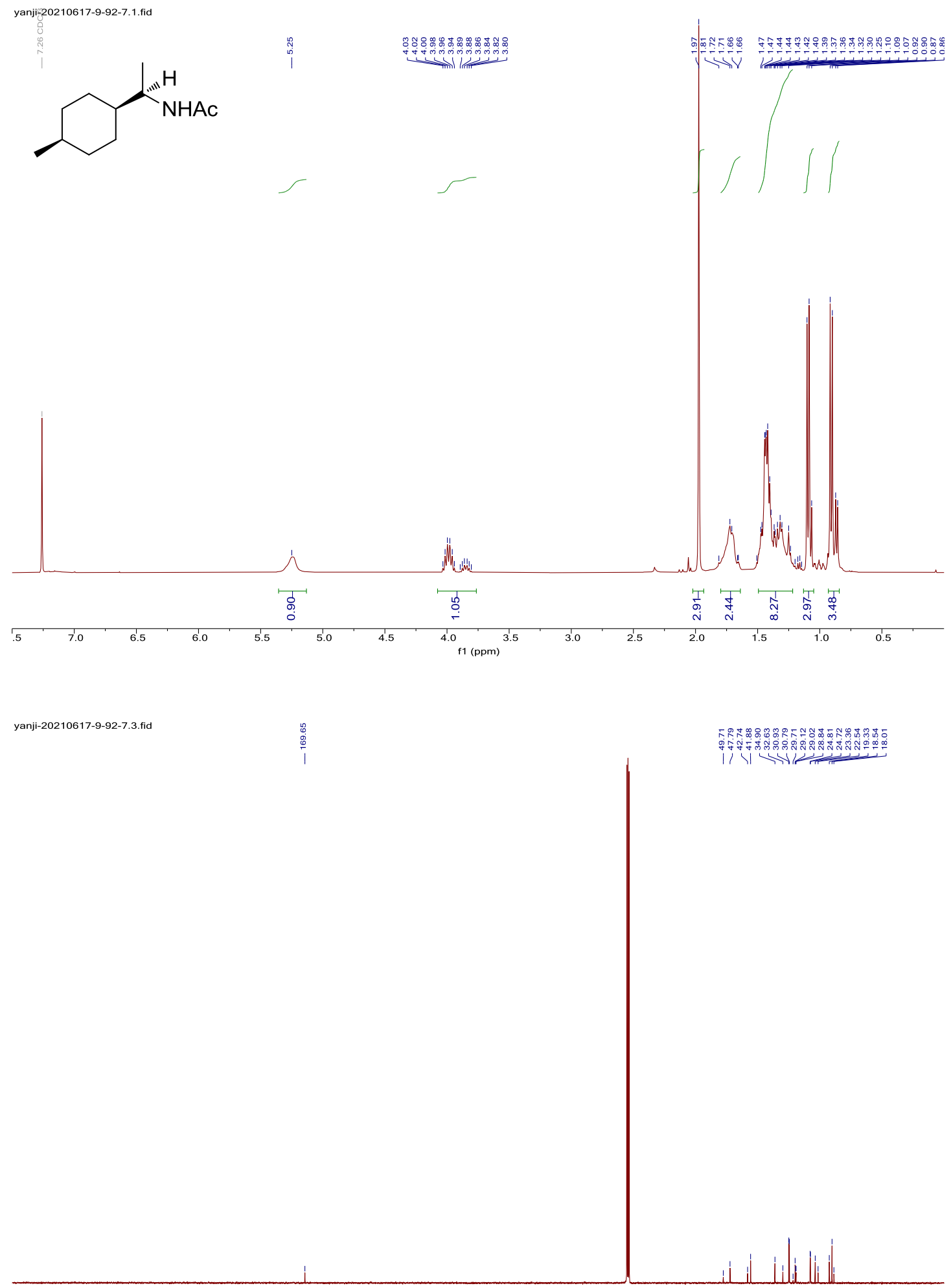

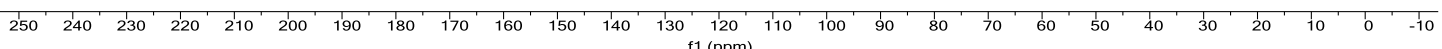


<smiles>CCNC(C)(C)C1CCC(C(C)=O)CC1</smiles>
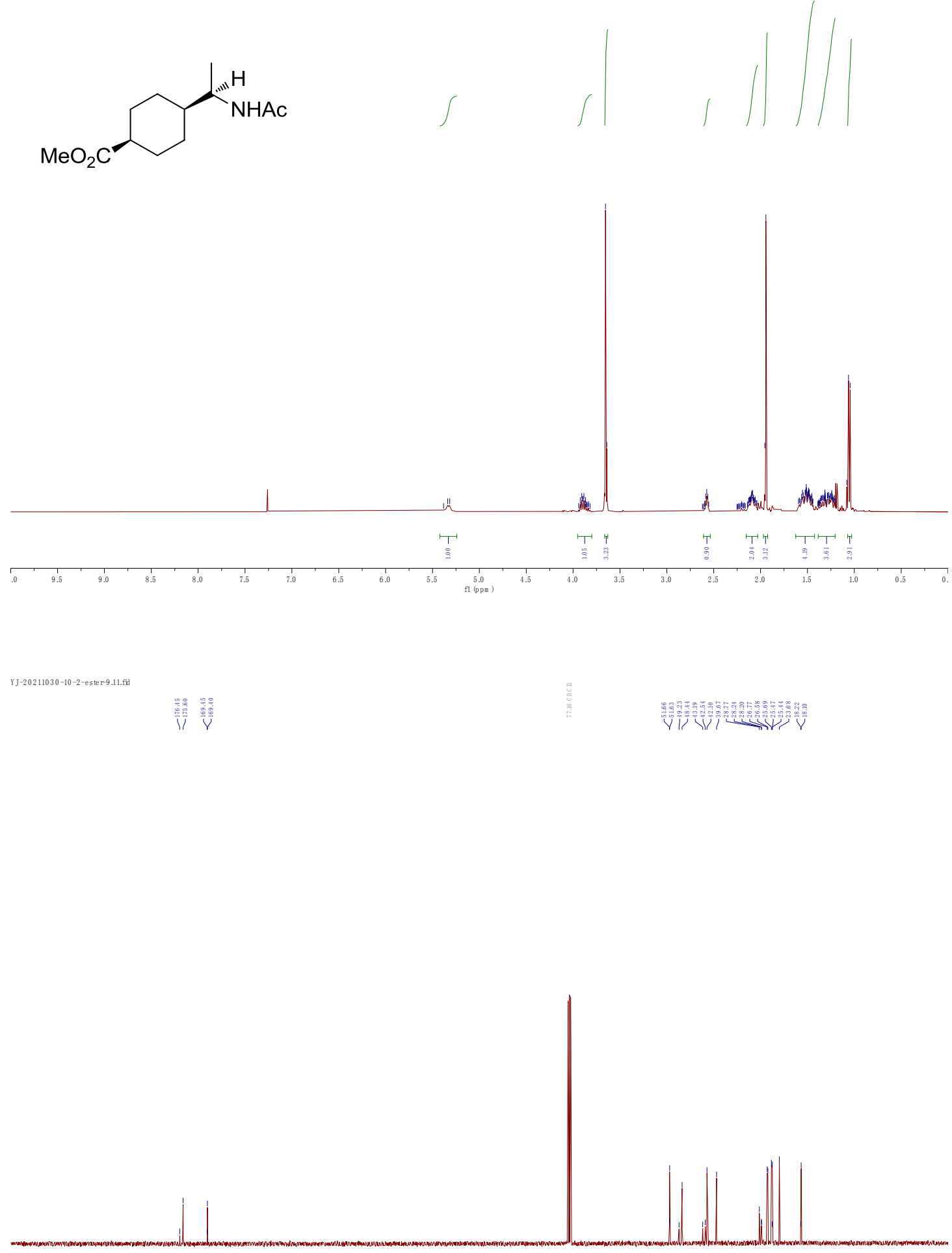


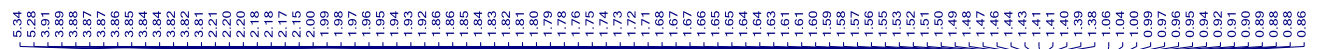<smiles>CC1CCC(C(=O)N[C@H](C)C2CCCCC2)CC1</smiles>

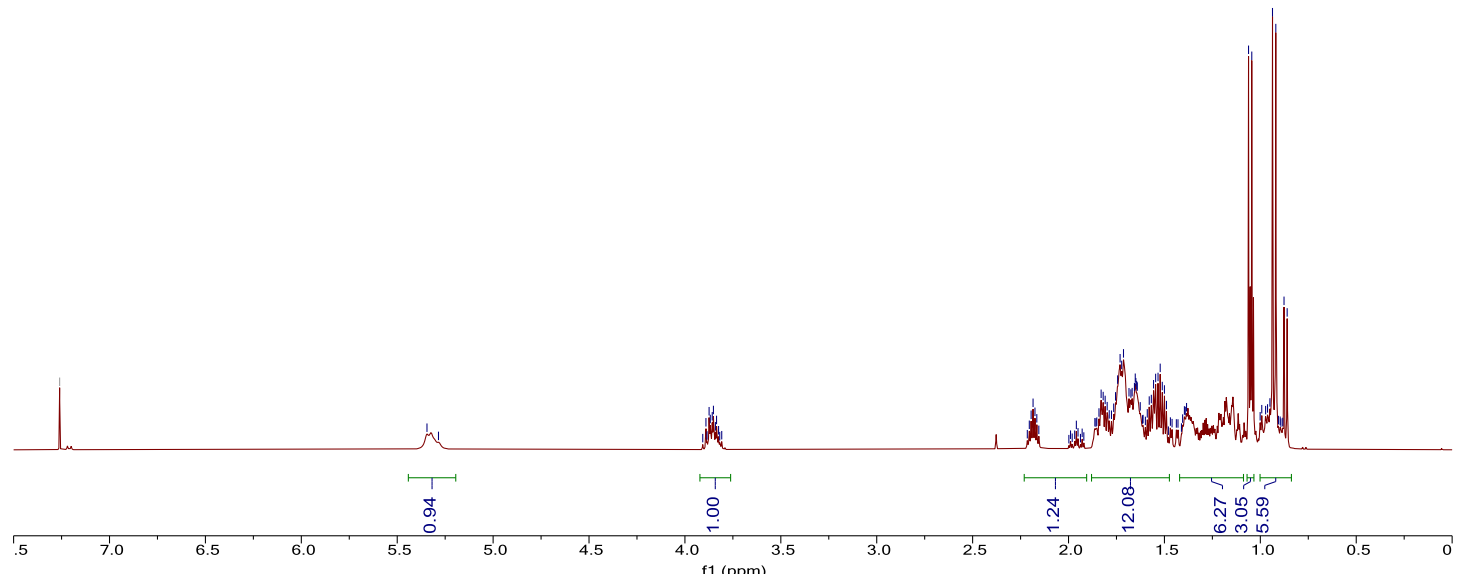

YJ-20210611-9-88-5-P.11.fid

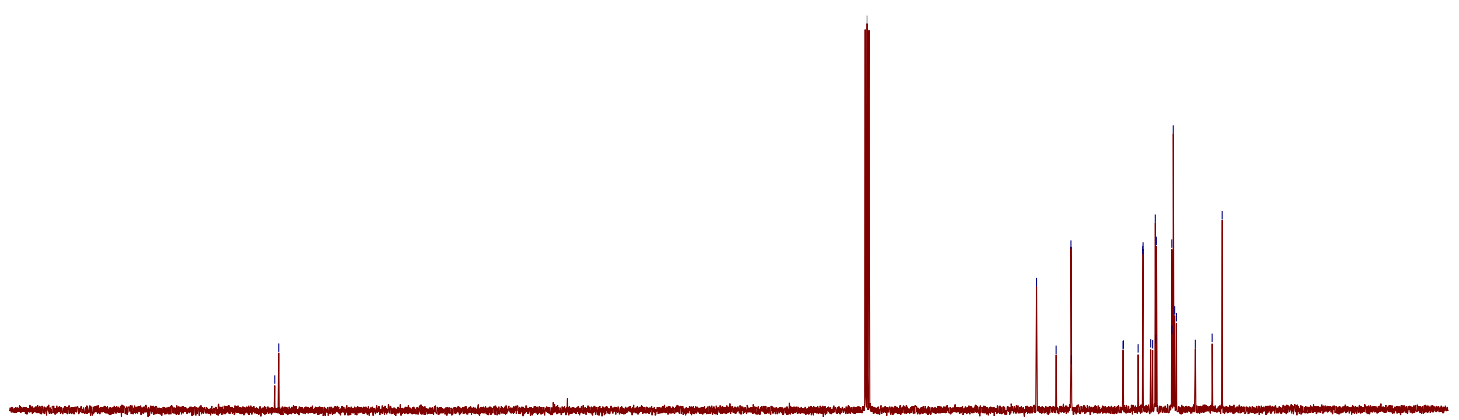

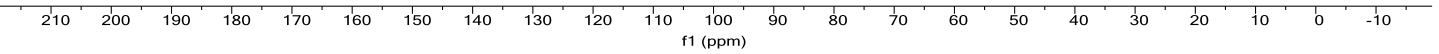



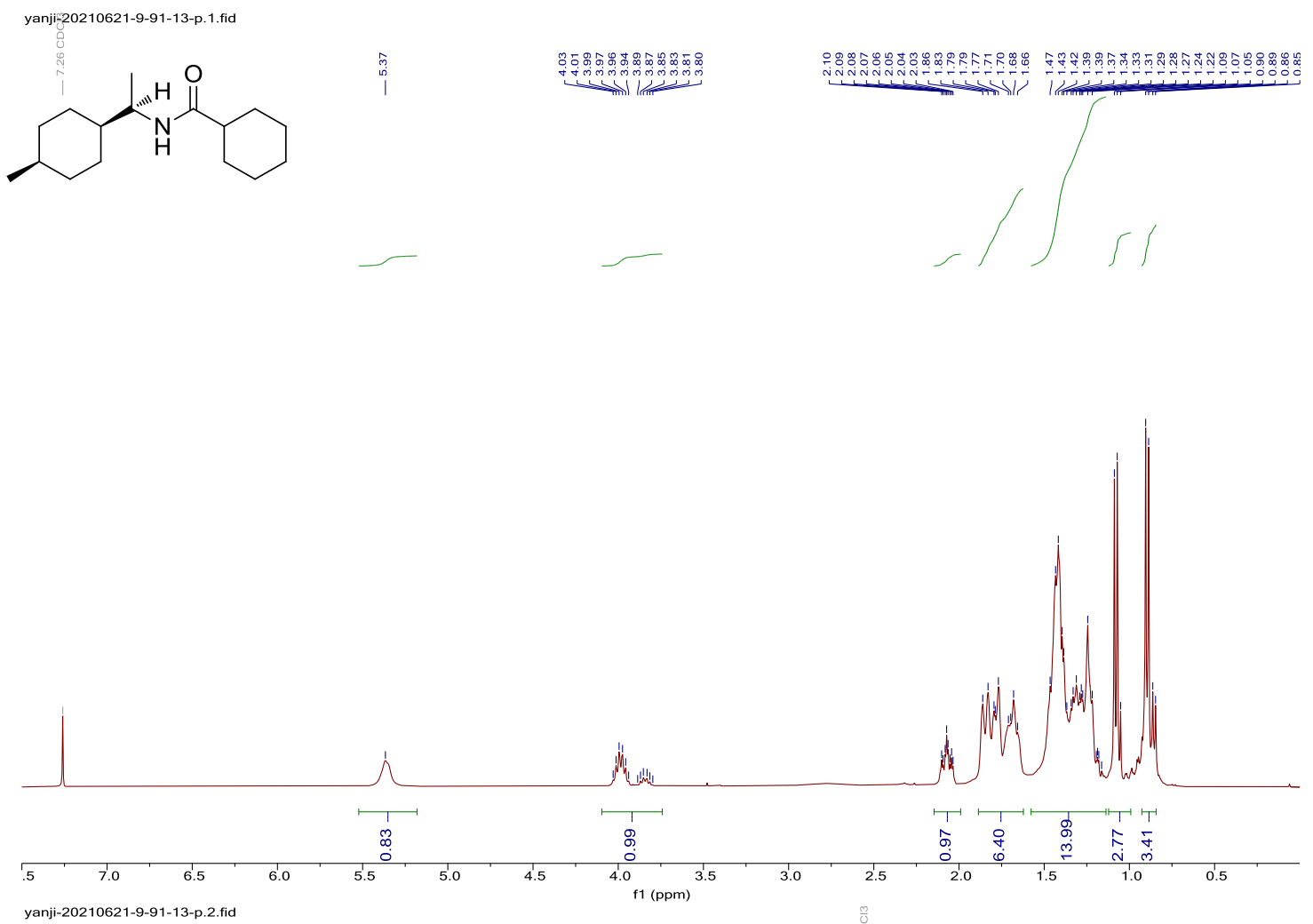
yanji-20210621-9-91-13-p.2.fid

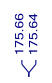

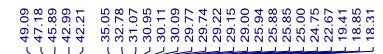
ly 11

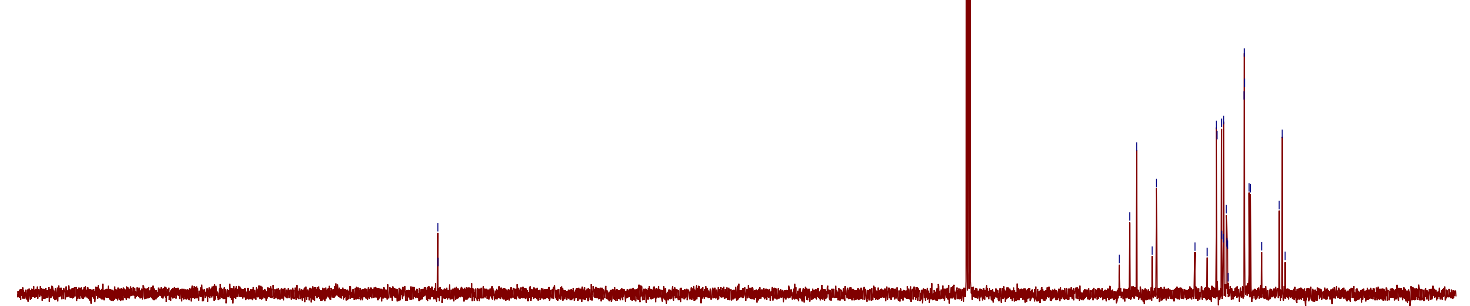

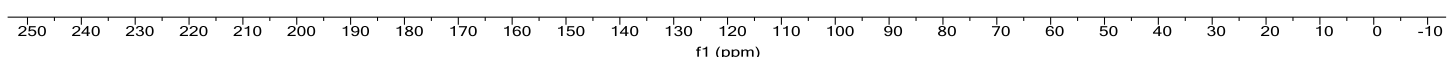




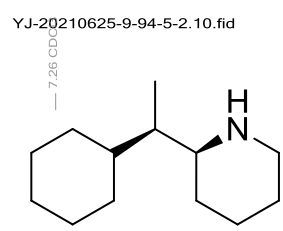

$\underbrace{\text { no }}$

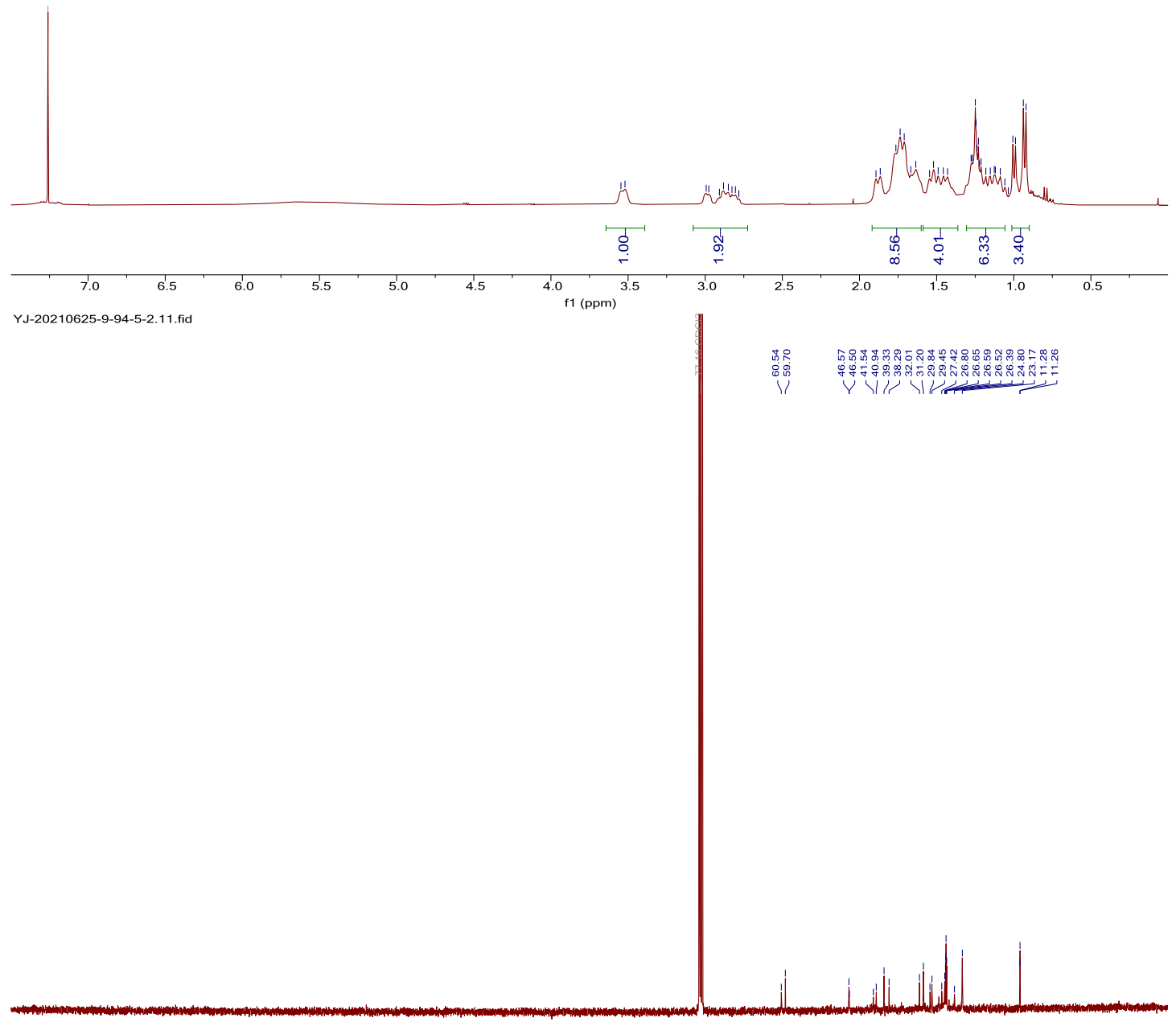

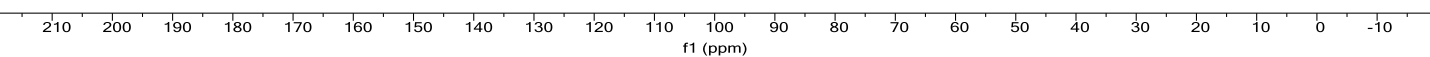




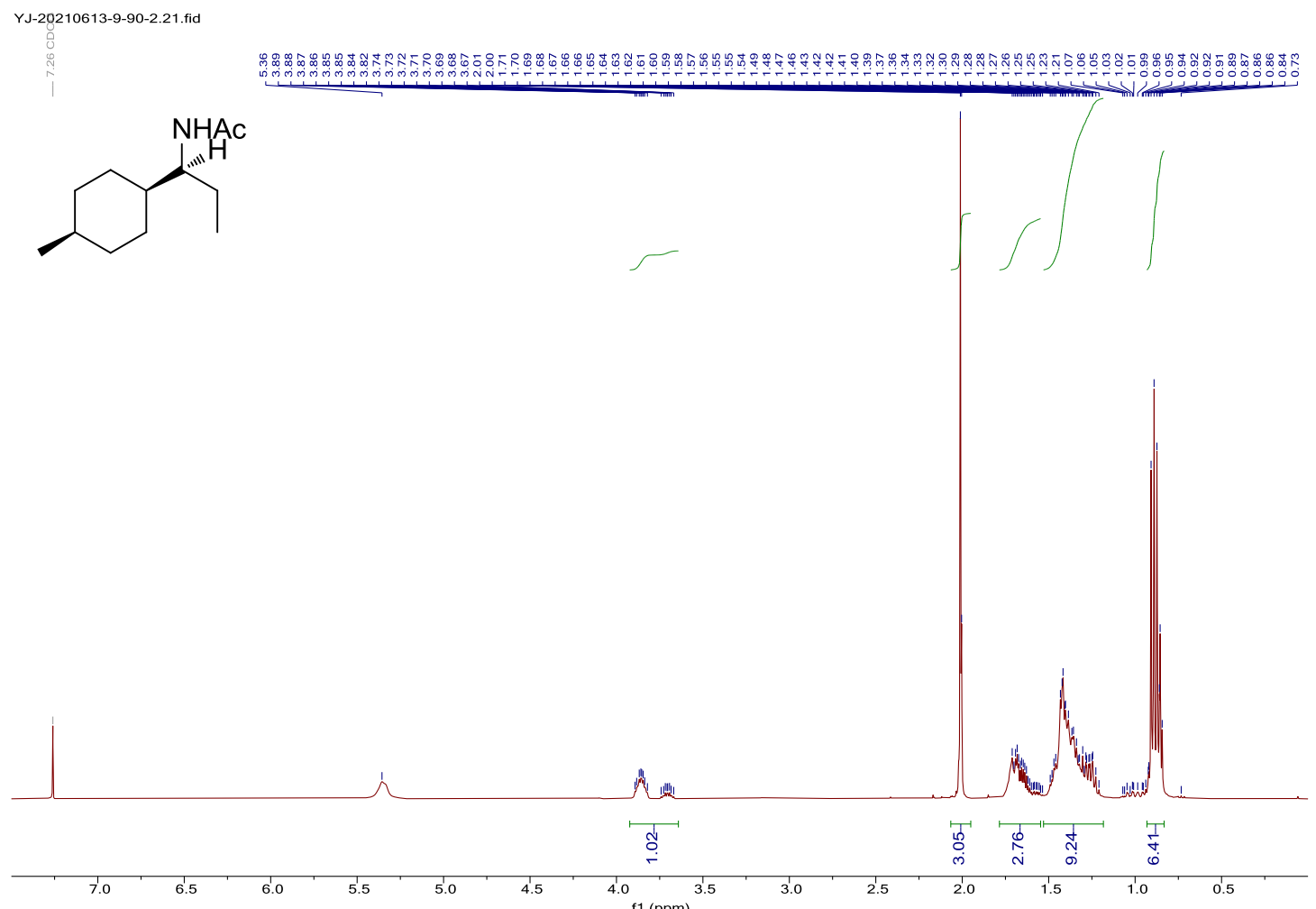

YJ-20210613-9-90-2.22.fid

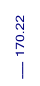

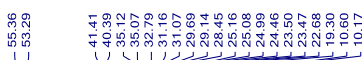

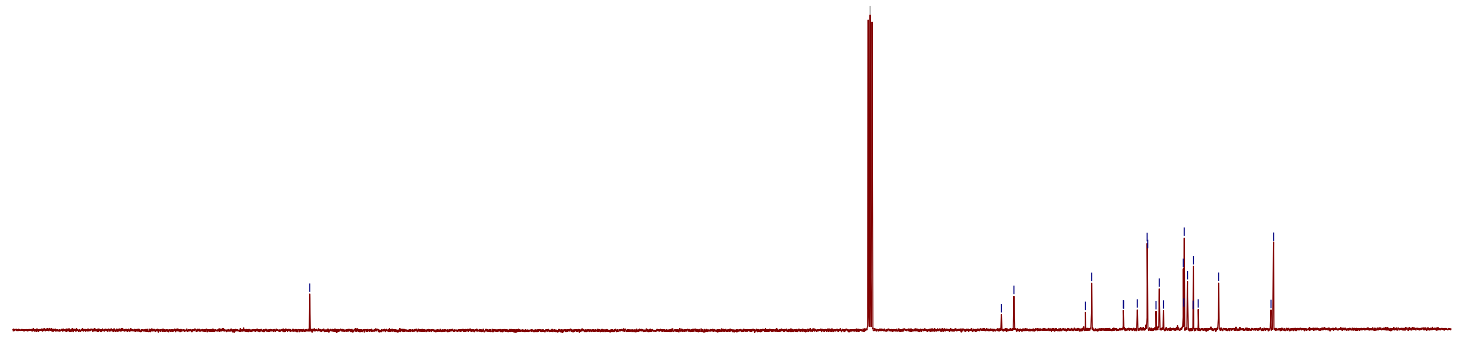

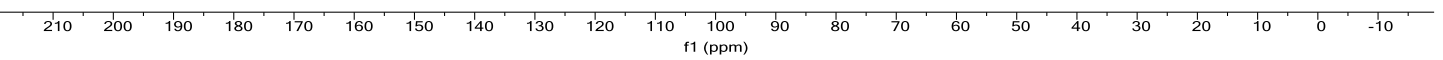




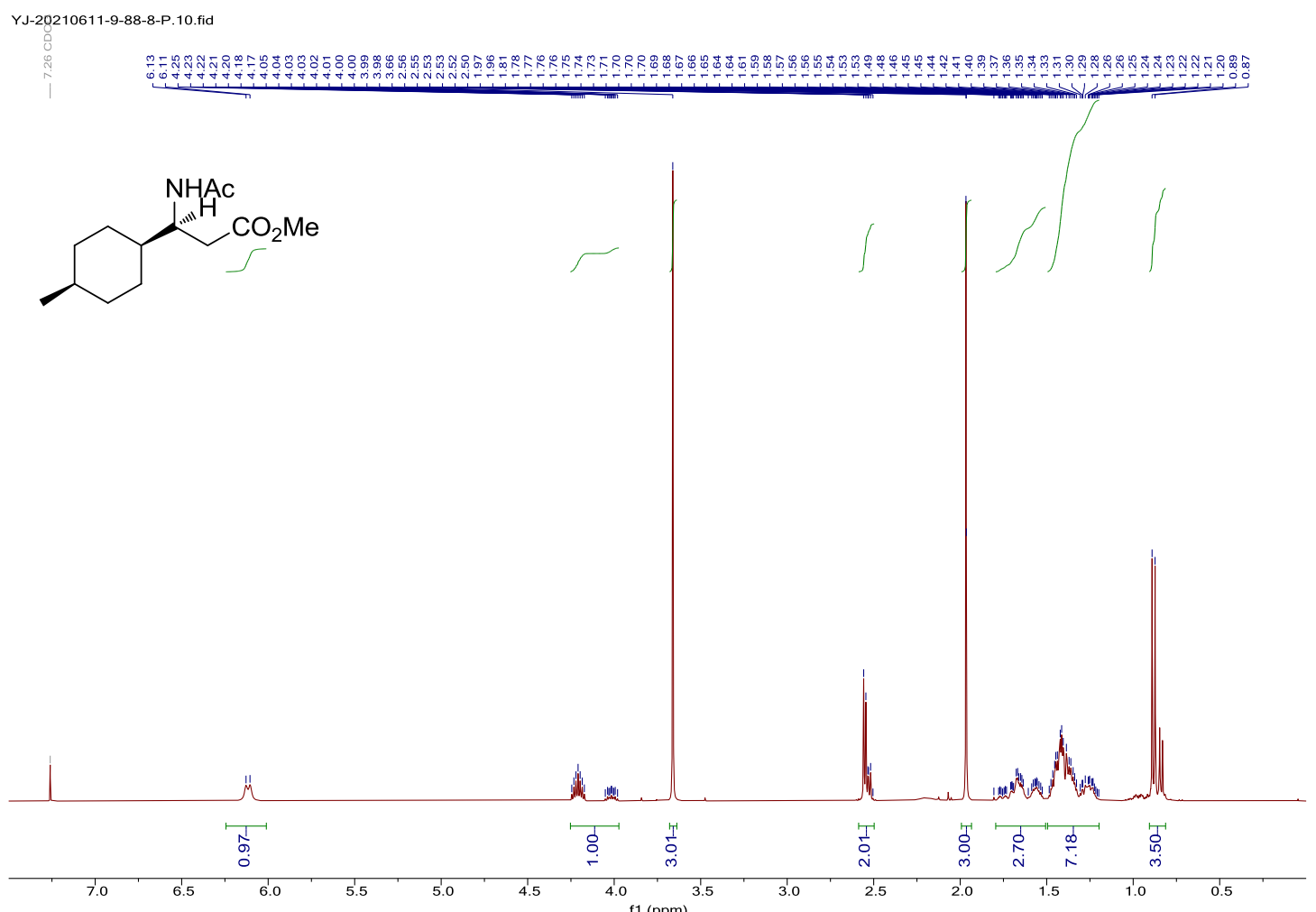

YJ-20210611-9-88-8-P.11.fid

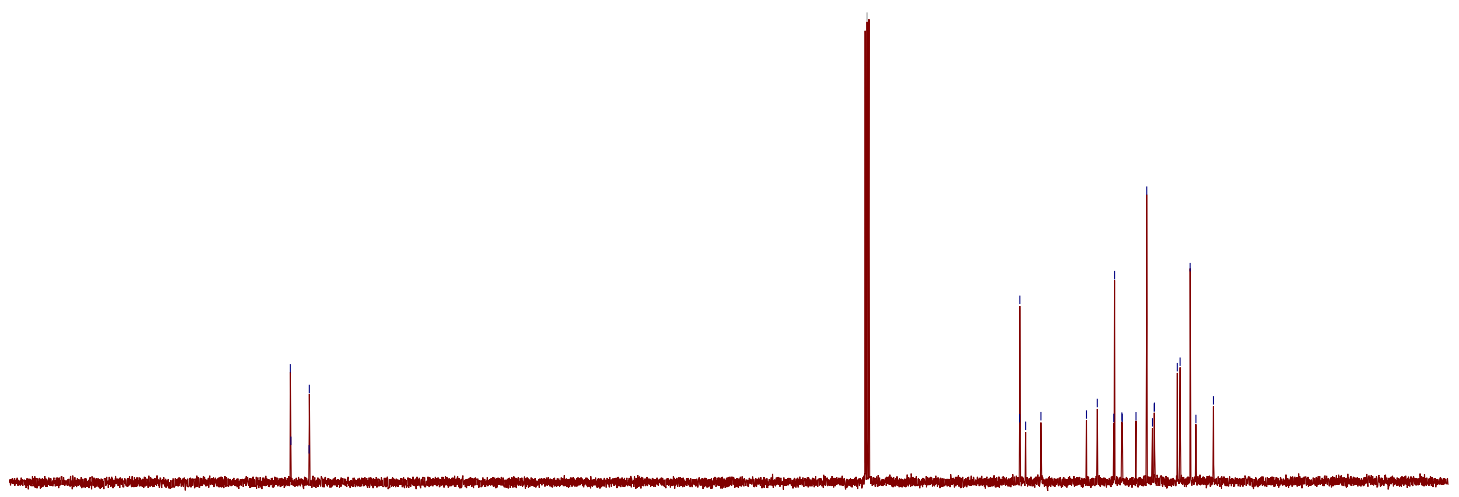

\begin{tabular}{lllllllllllllllllllllll}
\hline 210 & 200 & 190 & 180 & 170 & 160 & 150 & 140 & 130 & 120 & 110 & $\begin{array}{c}100 \\
\mathrm{f} 1(\mathrm{ppm})\end{array}$ & 90 & 80 & 70 & 60 & 50 & 40 & 30 & 20 & 10 & 0 & -10
\end{tabular} 
<smiles>COC1CCC([C@](N)(CC(C)=O)C(C)C)CC1</smiles><smiles>CCC(C)C1C=CCCC1</smiles>
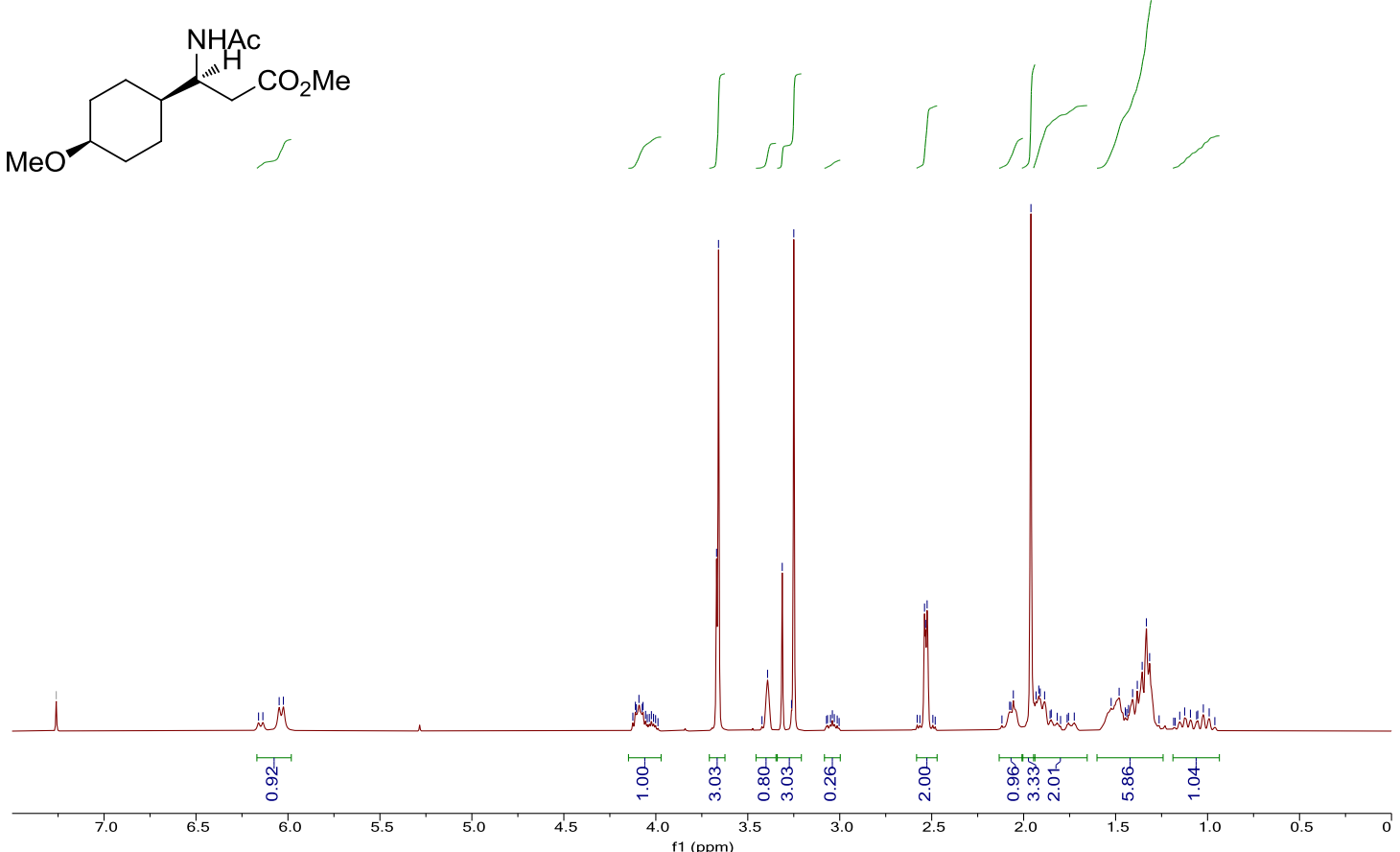

yanji-20210611-9-88-9-P.2.fid

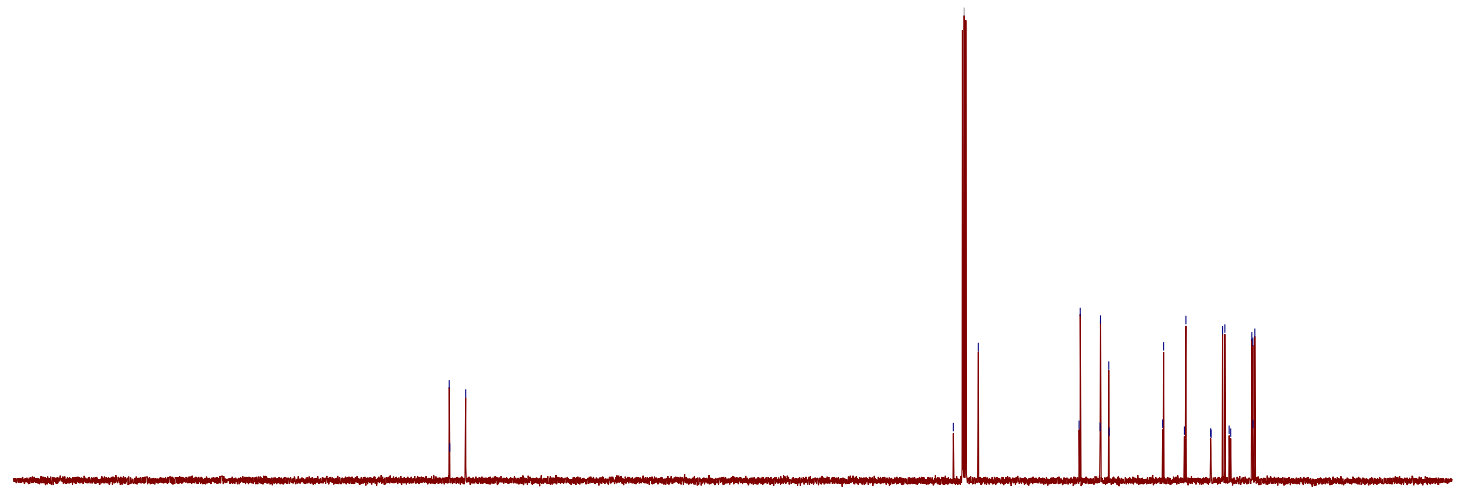

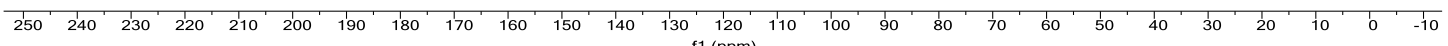


fiwu-210619-206-87b2p.10.fid

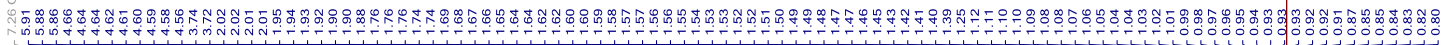<smiles>COC(=O)C(N)(N)C[C@H]1CC[C@H](C(C)(C)C)CC1</smiles>
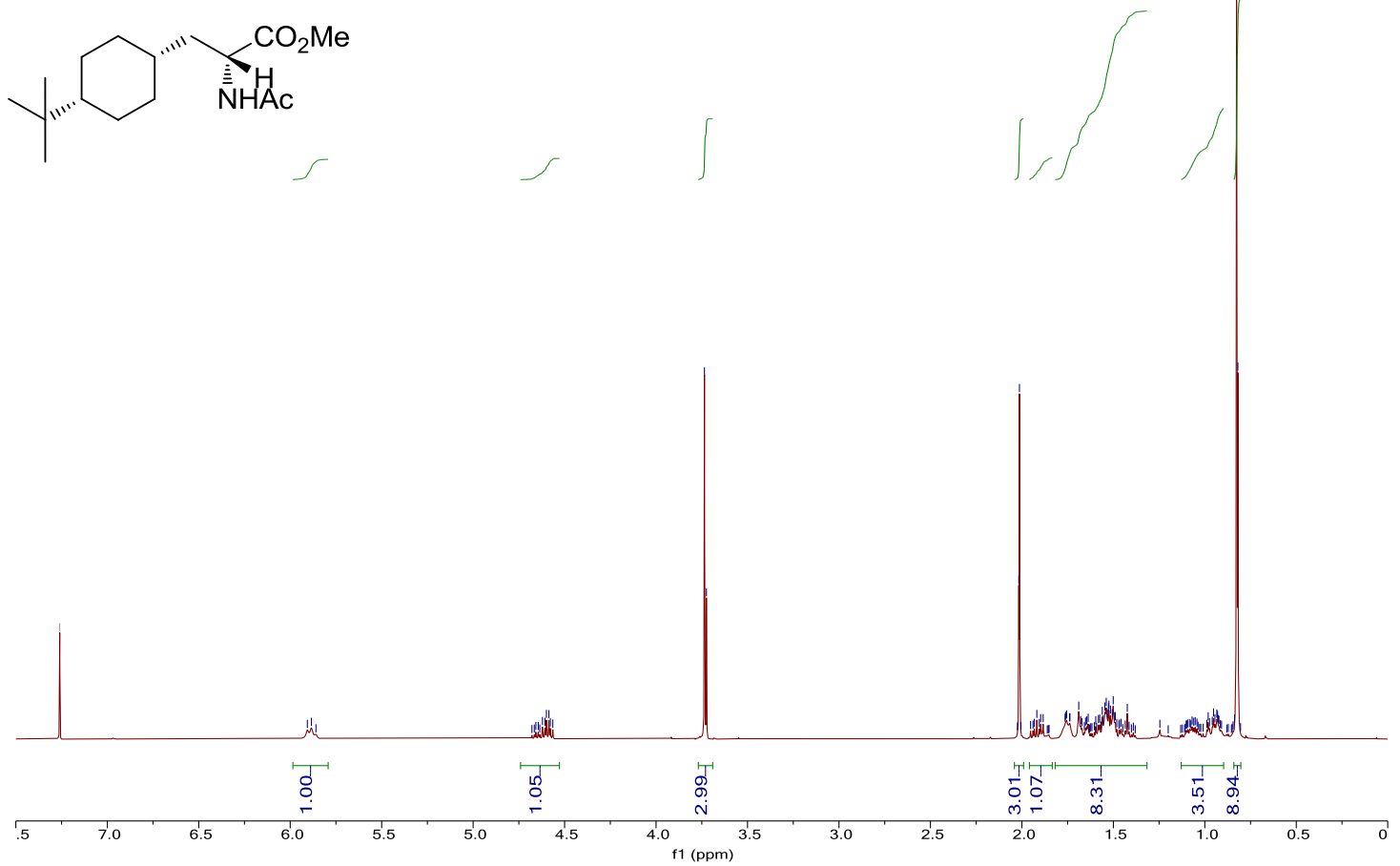

hwu-210619-206-87b2p.11.fid

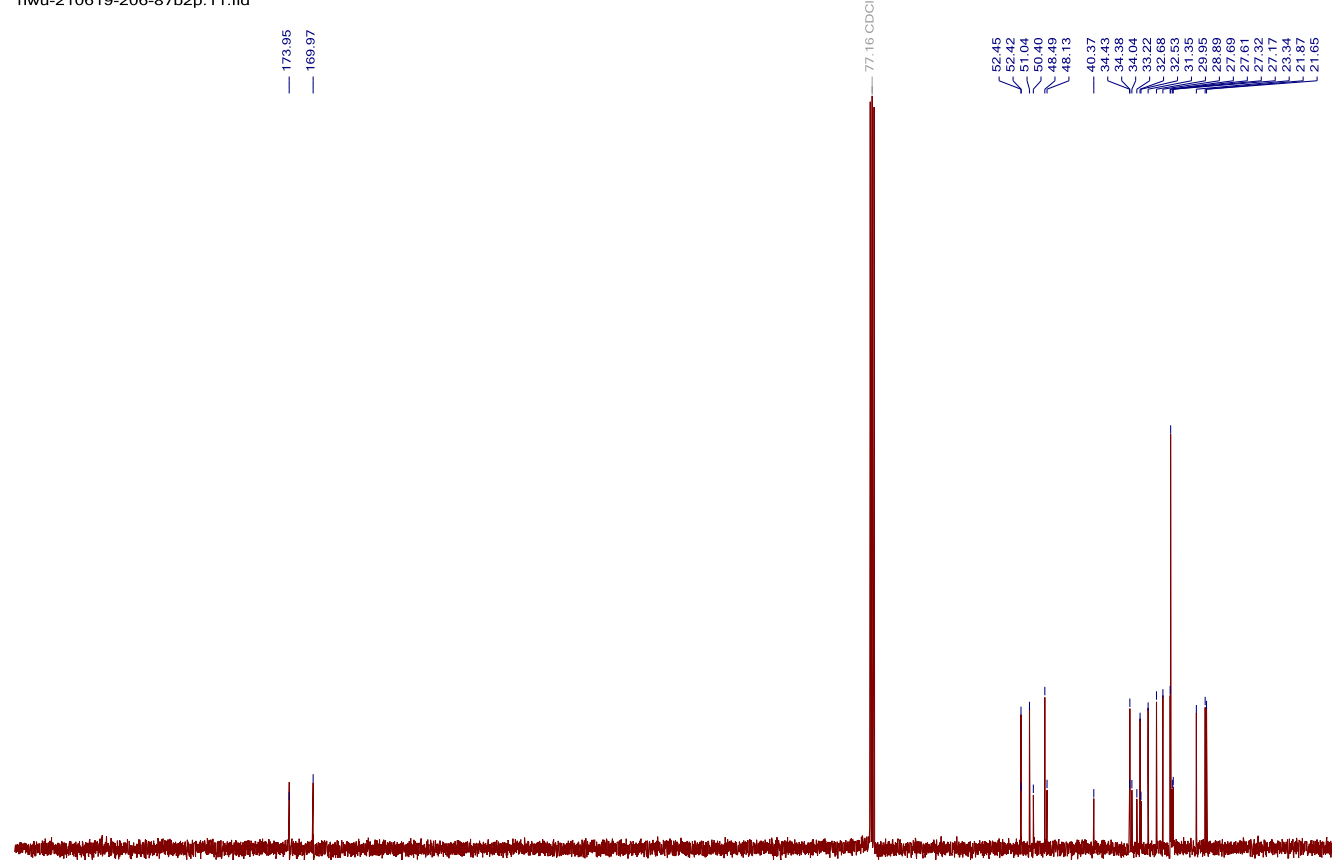

$\underbrace{}_{11}$

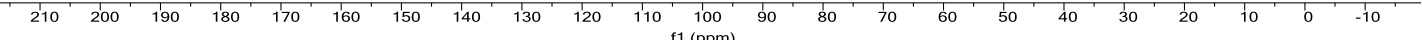


<smiles>COC(=O)C(N)(C[C@H]1CC[C@H](C)CC1)C(=O)OC</smiles>

NHAC

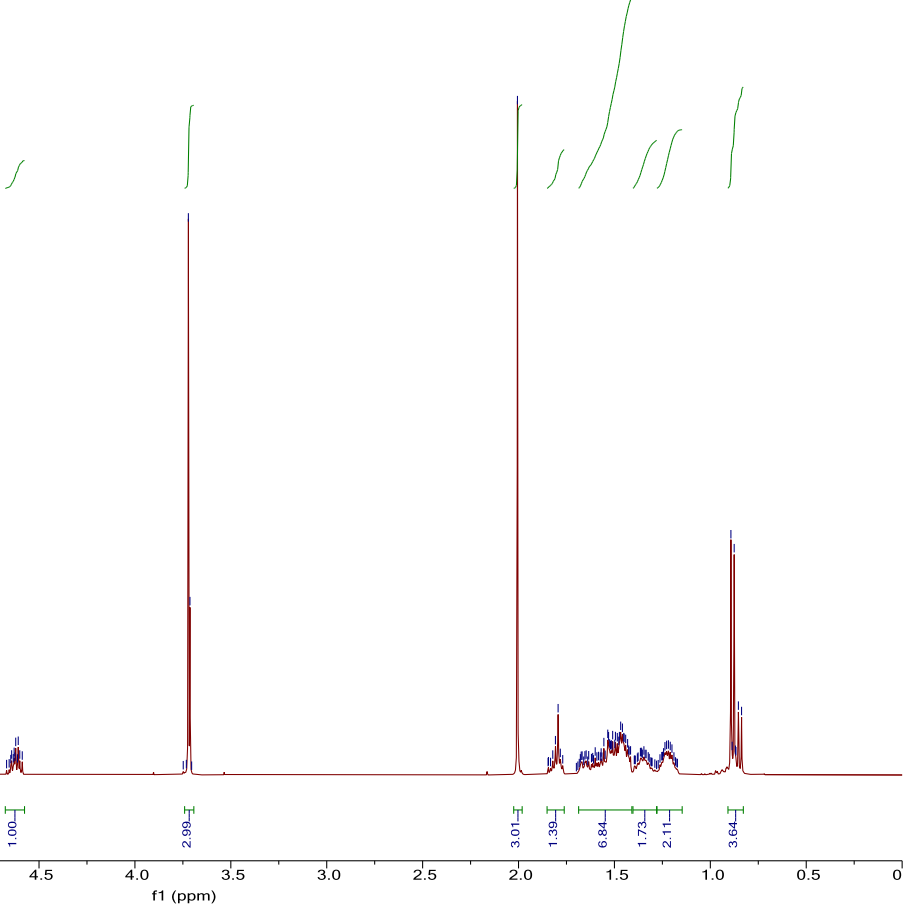

hwu-210602-206-73gp.11.fid

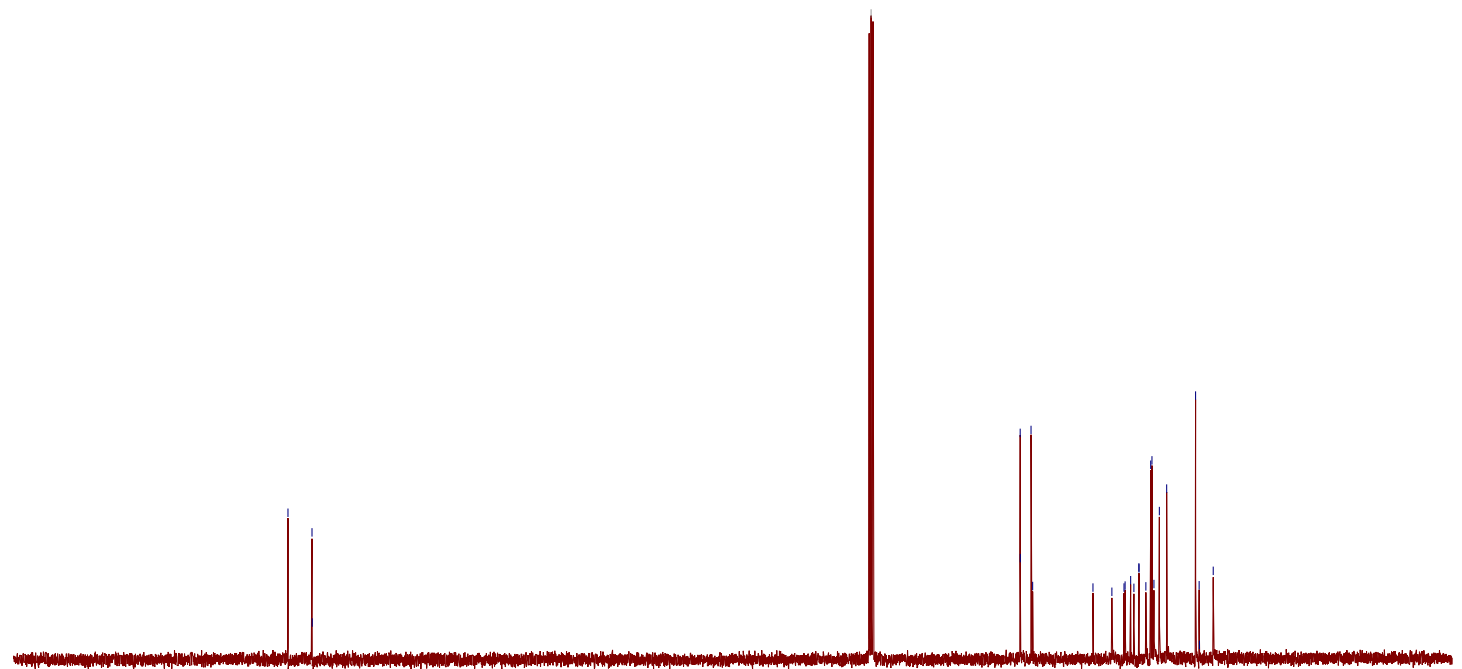

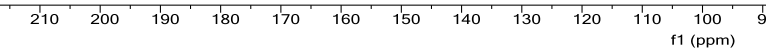




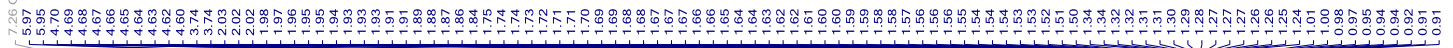<smiles>COC(=O)[C@@](N)(CN)CC1CCC(C(F)(F)F)CC1</smiles><smiles>C1CCC1</smiles>
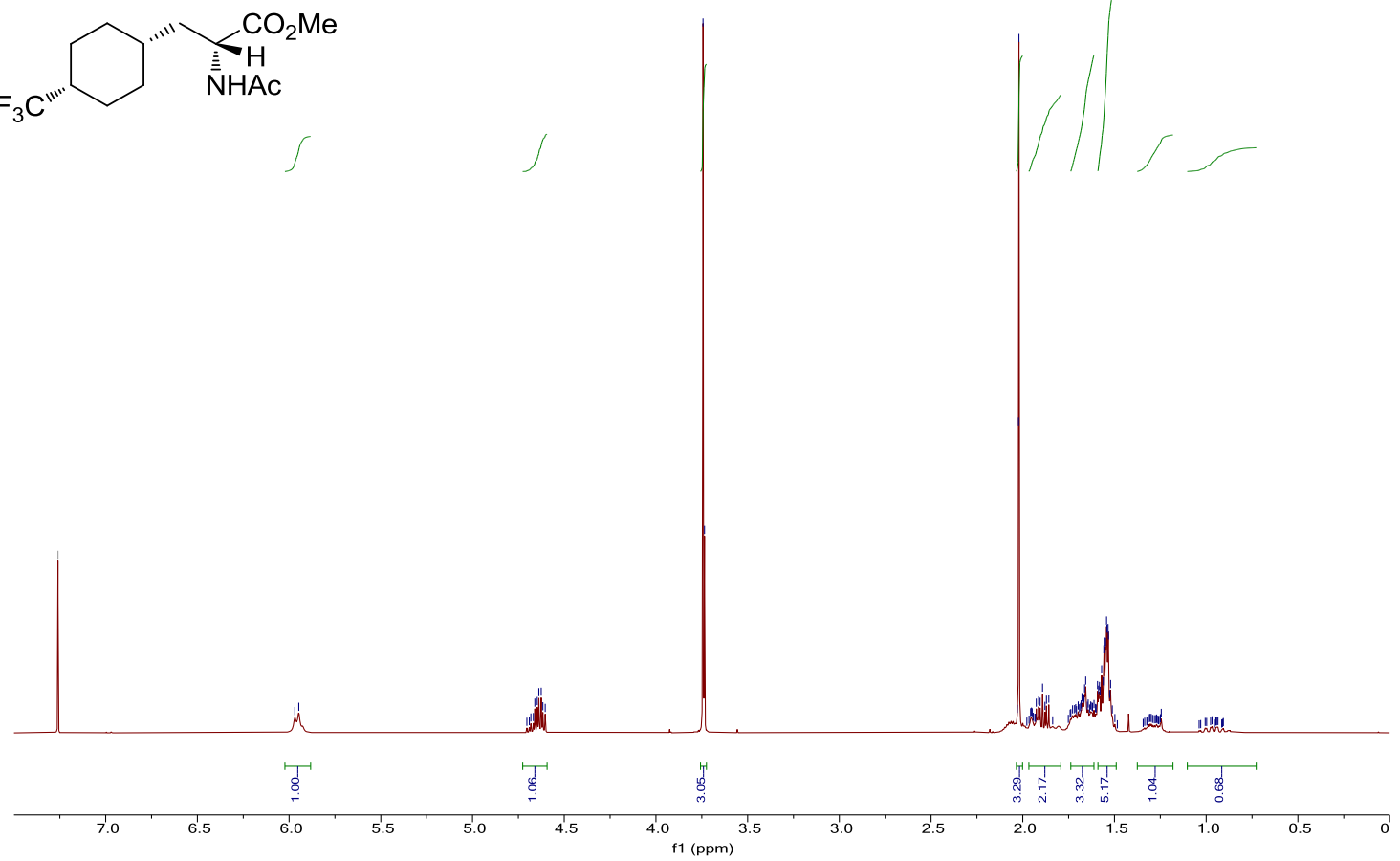

hwu-210619-206-74b2p.12.fid

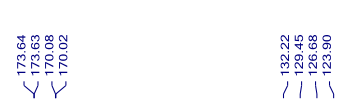

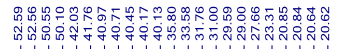

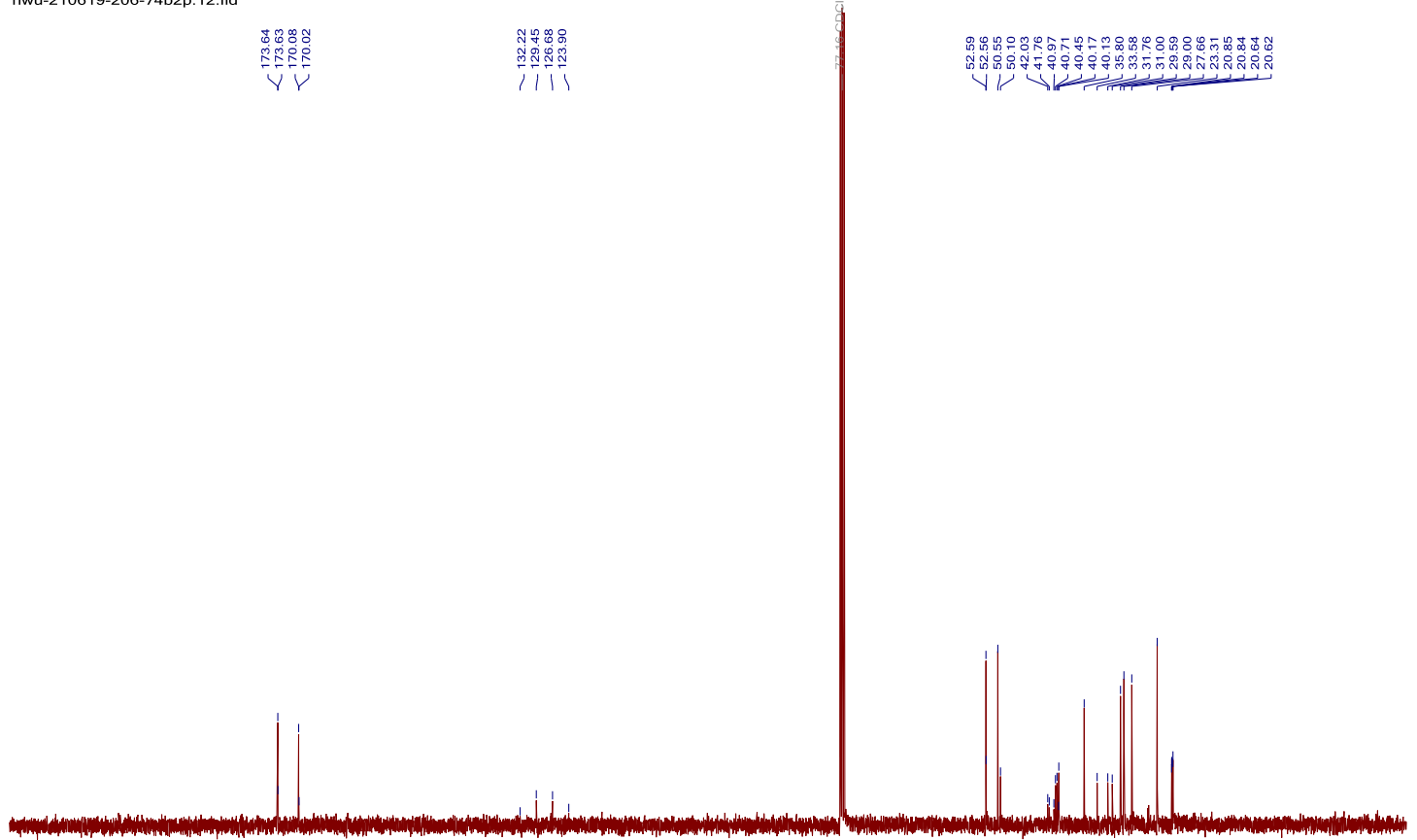

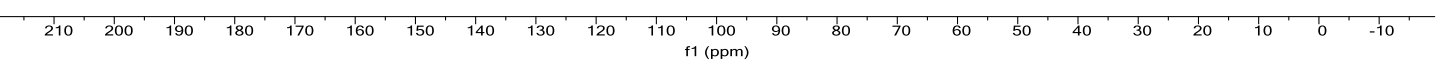




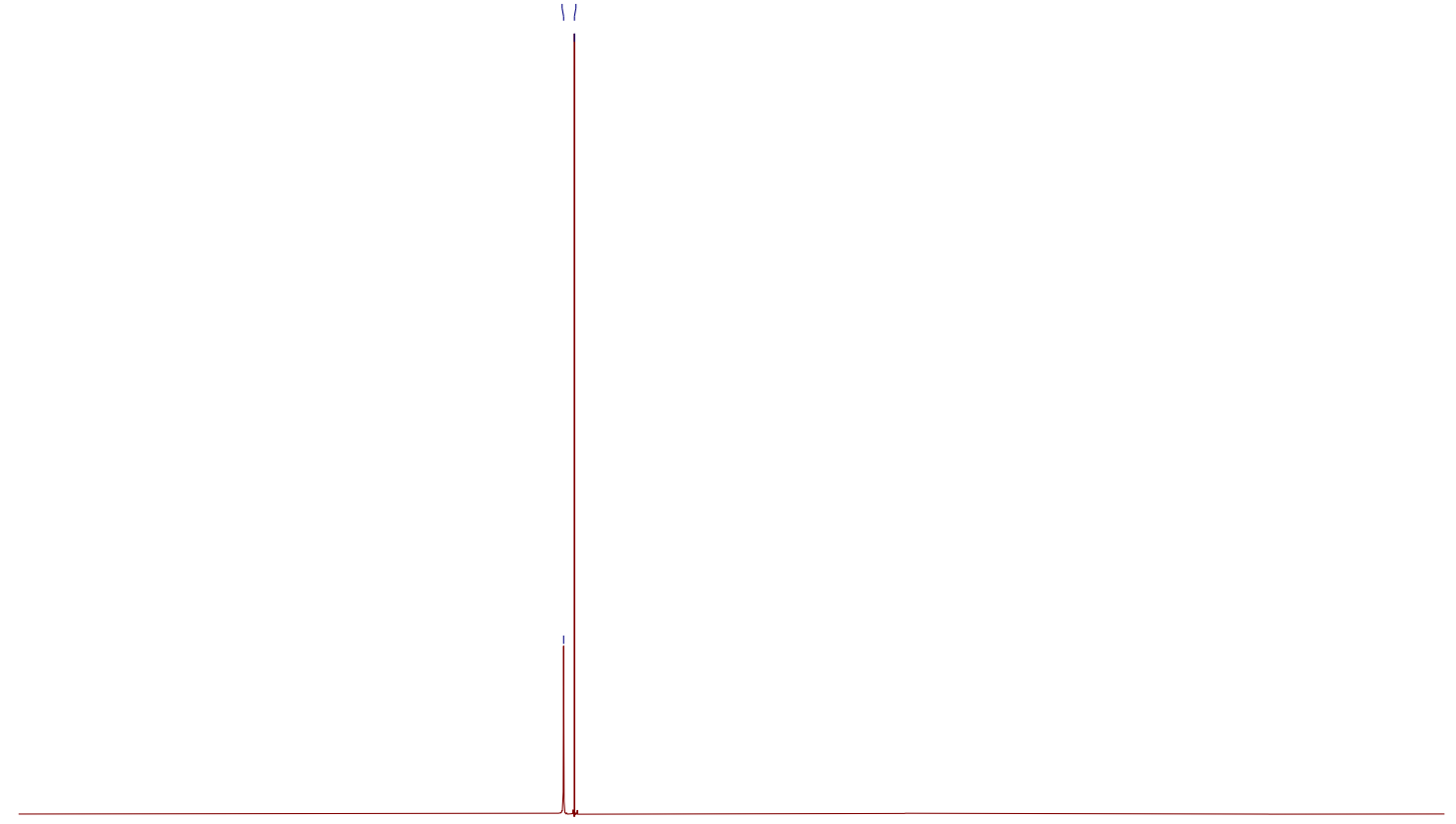




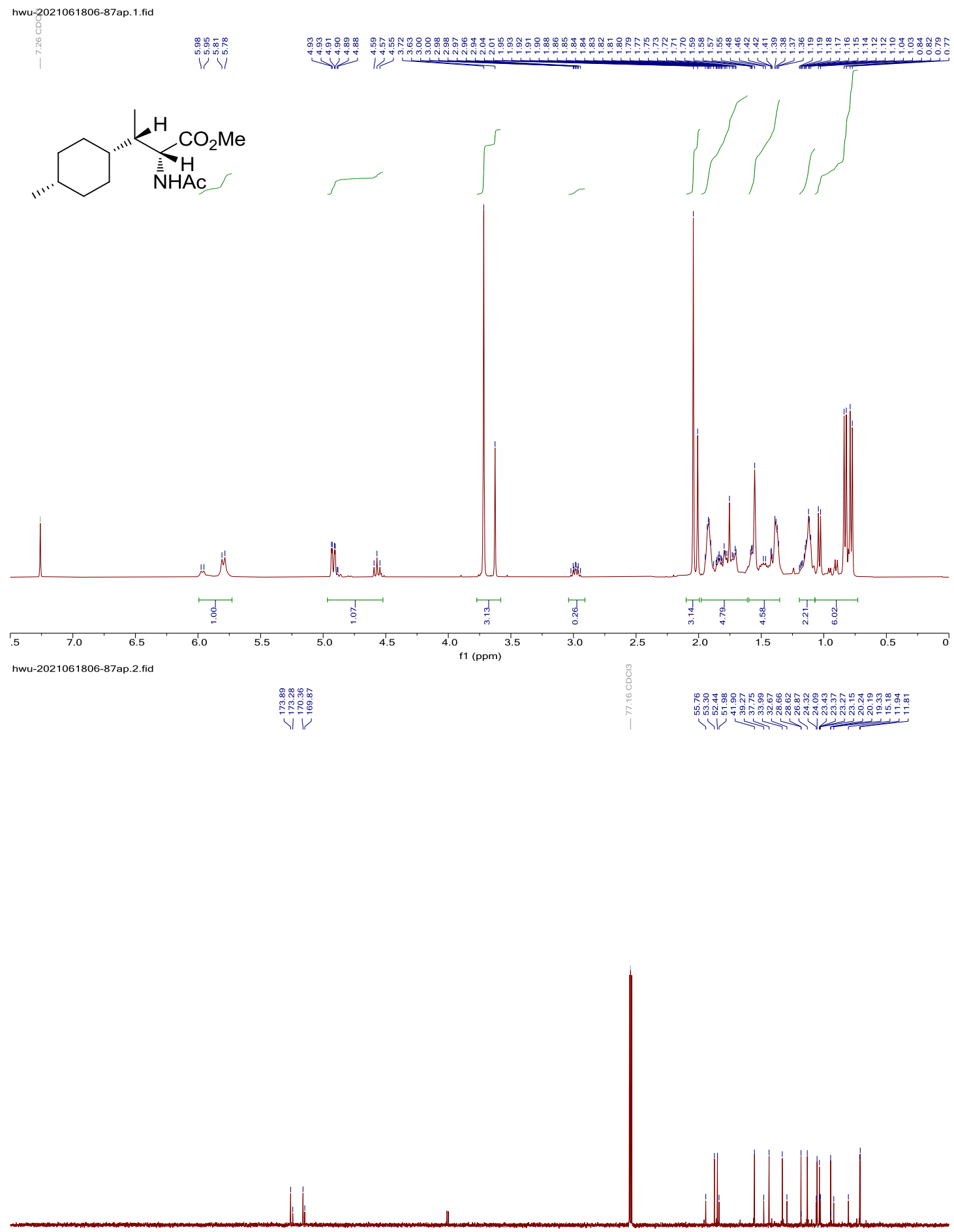

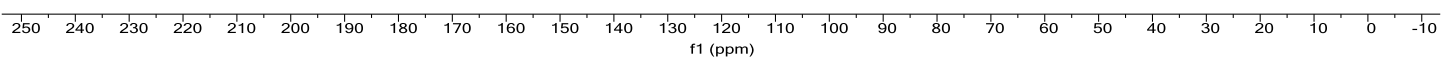




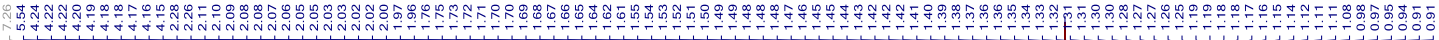<smiles>CC(=O)N[C@H]1CC[C@@H]2CCCC[C@H]21</smiles>

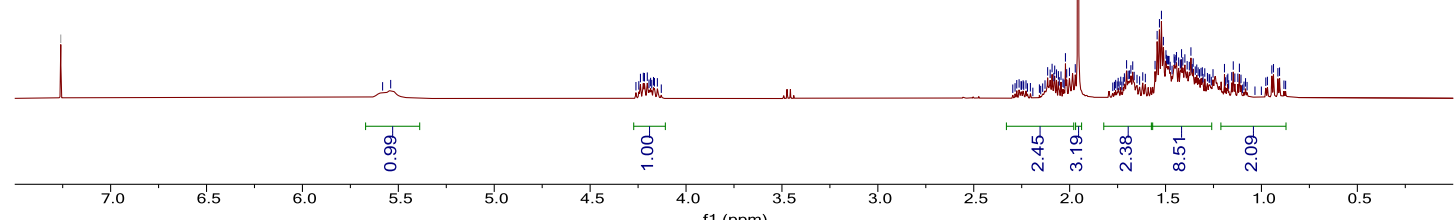
YJ-20210618-9-92-9.11.fid

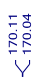

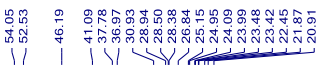

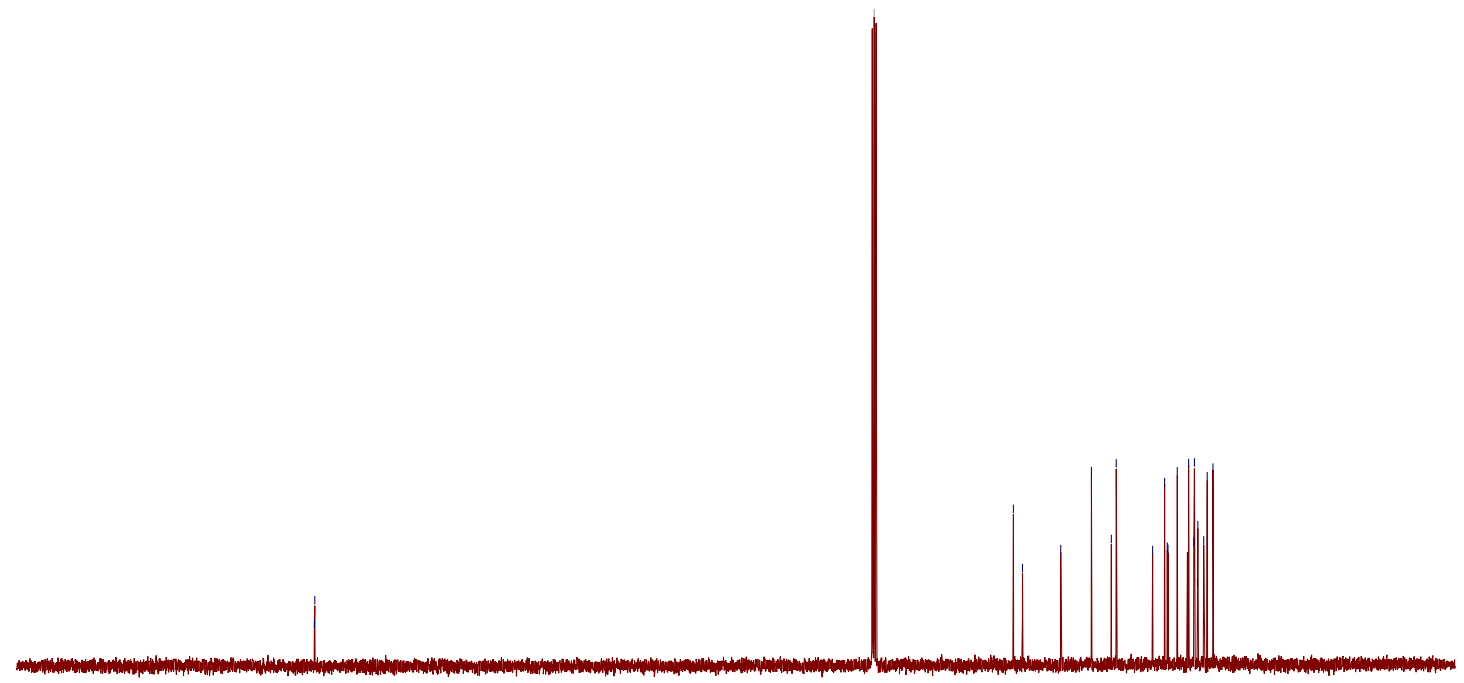

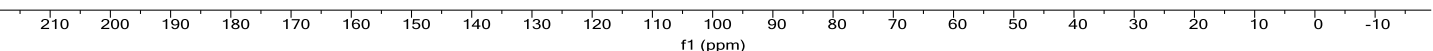




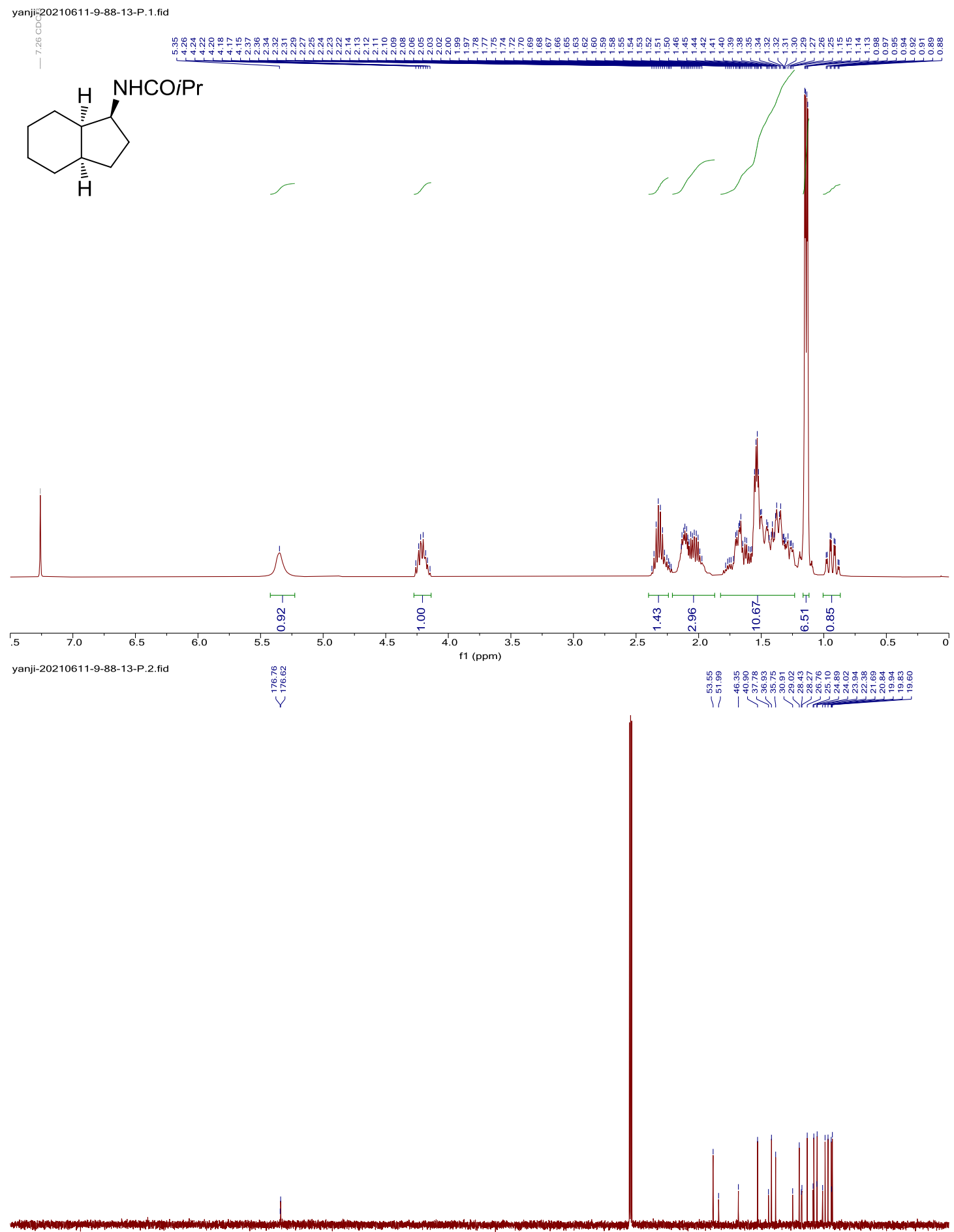

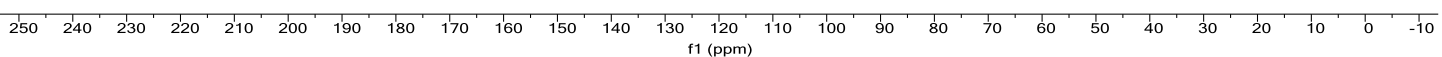


<smiles>CCCCC(=O)N[C@H]1CC[C@@H]2CCCC[C@@H]21</smiles>
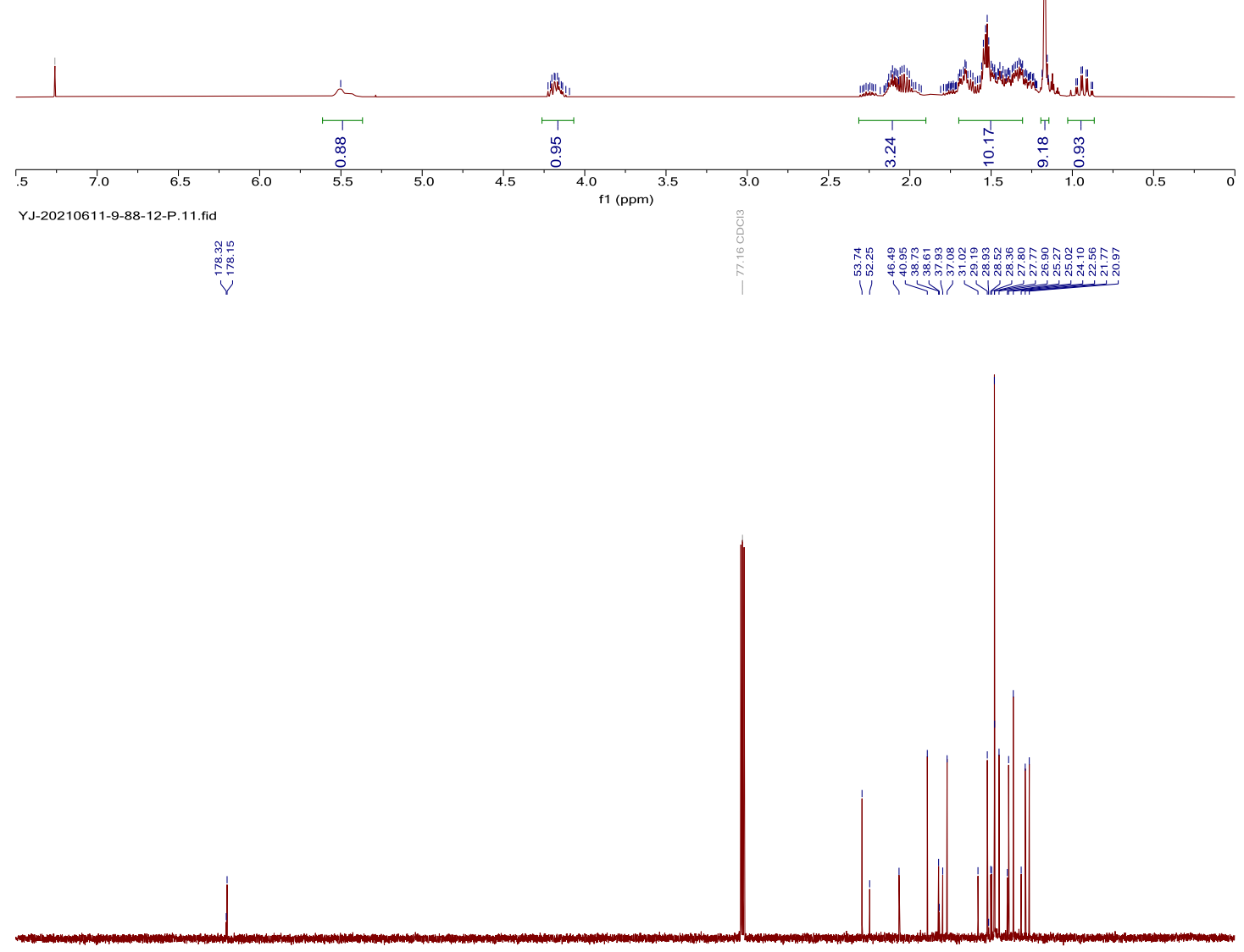

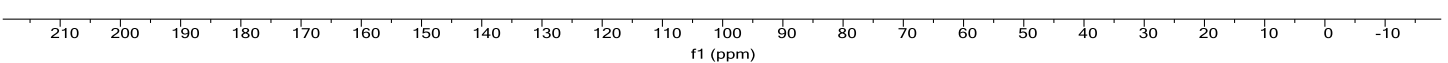




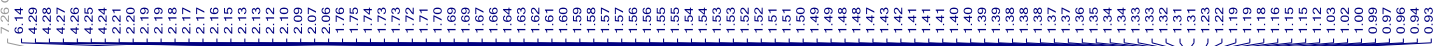
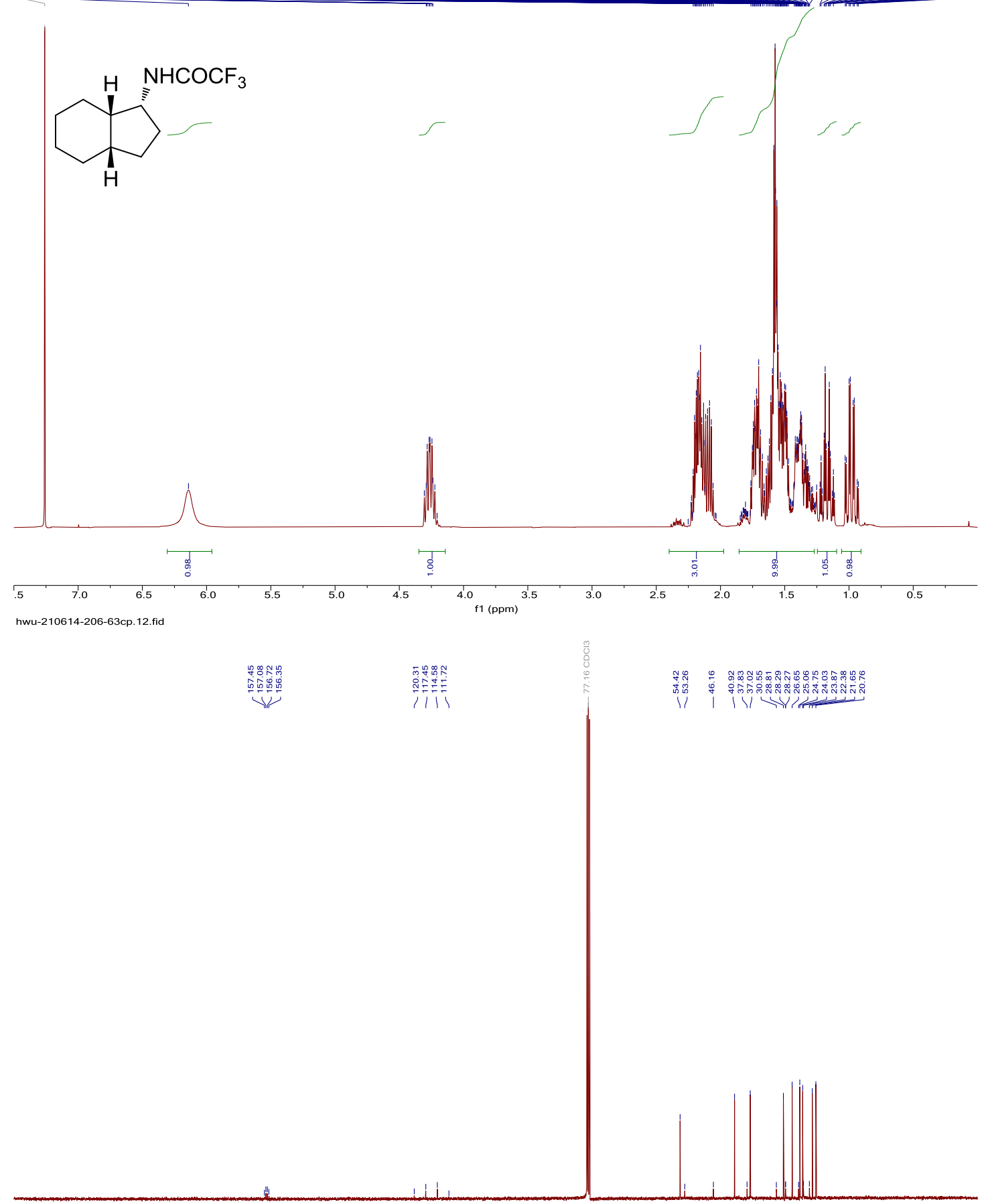

$\begin{array}{lllllllllllllllllllllllllllllllll}210 & 200 & 190 & 180 & 170 & 160 & 150 & 140 & 130 & 120 & 110 & 100 & 90 & 10 & 70 & 60 & 50 & 40 & 30 & 20 & 10 & 0 & -10\end{array}$ 


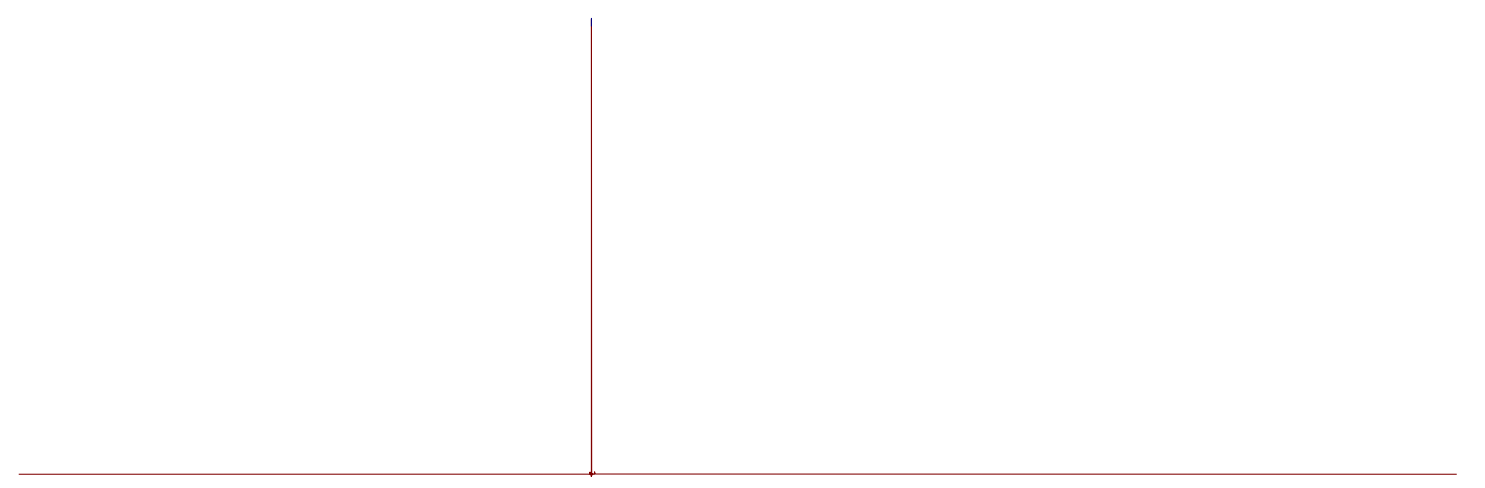

$\begin{array}{lllllllllllllllllllllll}10 & 0 & -10 & -20 & -30 & -40 & -50 & -60 & -70 & -80 & -90 & -100 & -110 & -120 & -130 & -140 & -150 & -160 & -170 & -180 & -190 & -200 & -210\end{array}$ 
fiwu-210612-206-79dp.10.fid

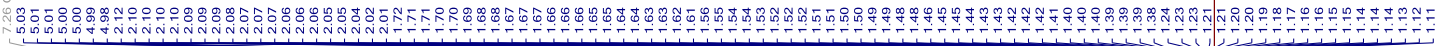<smiles>CCCCO[C@H]1CC[C@H]2CCCC[C@H]12</smiles>

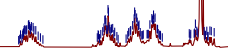

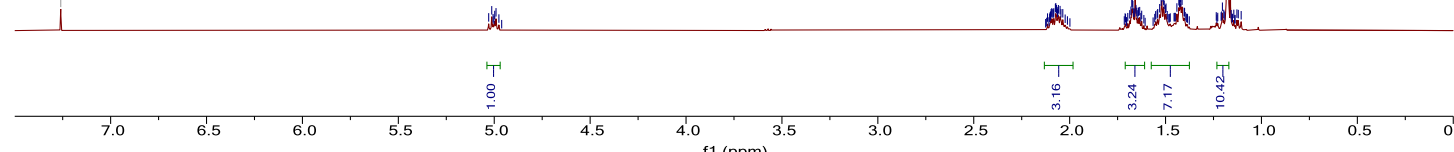

hwu-210612-206-79dp.11.fid
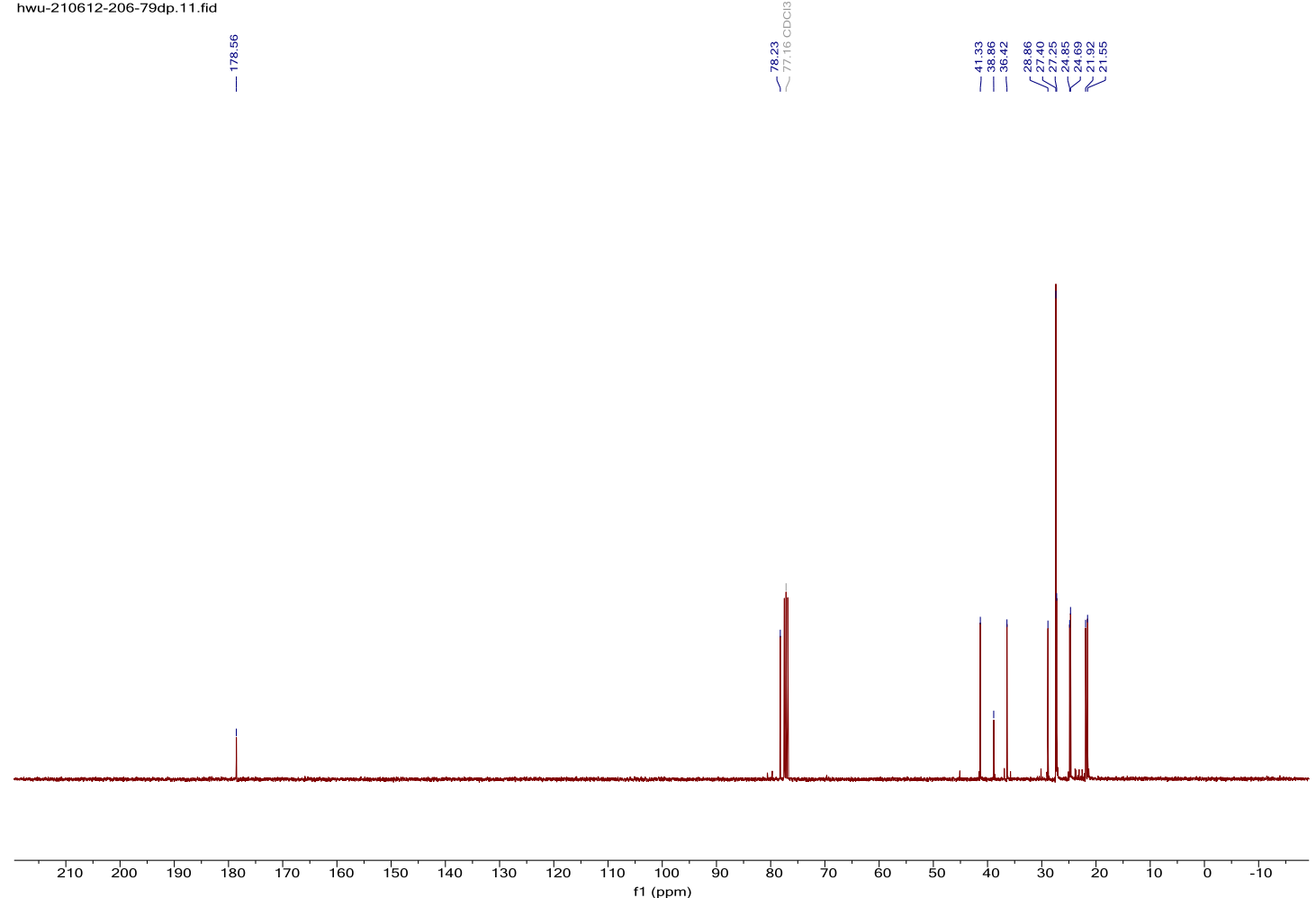
Gुwu-210604-206-73fp.10.fid

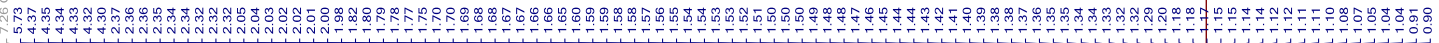

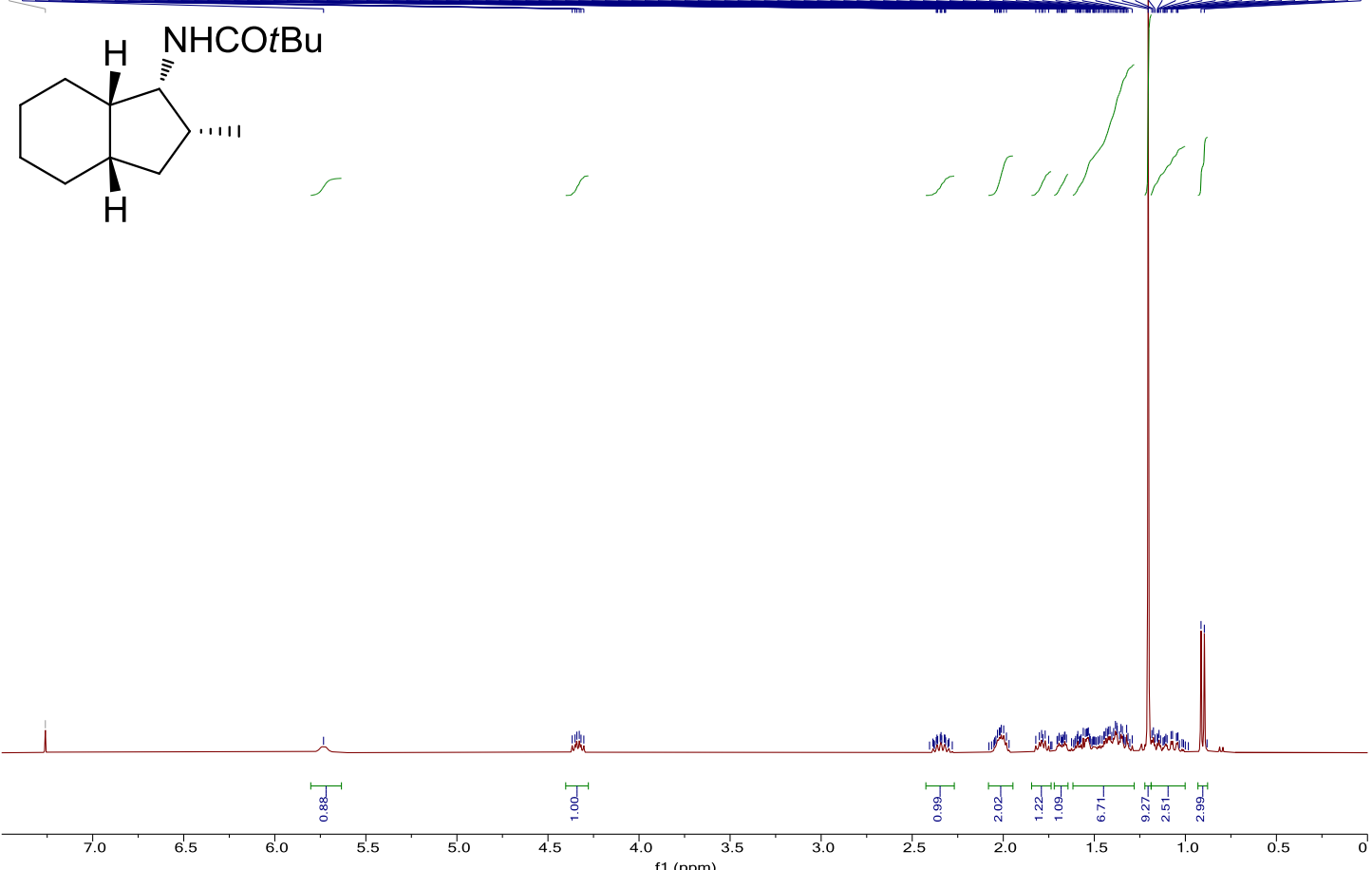

hwu-210604-206-73fp. 12.fid

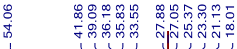

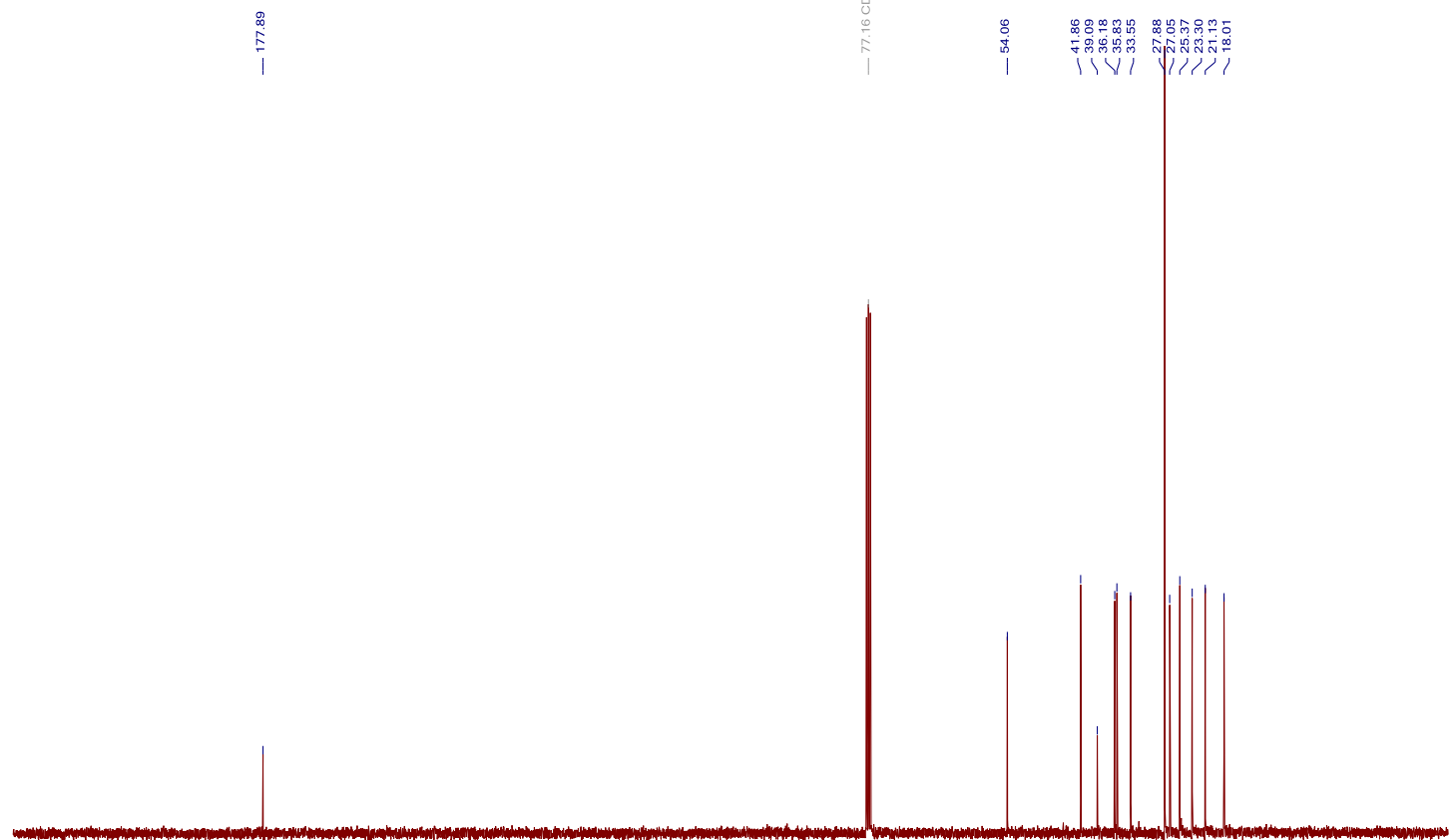

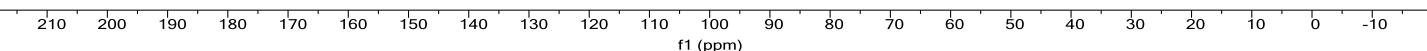


hwu-2021060406-73dp.1.fid

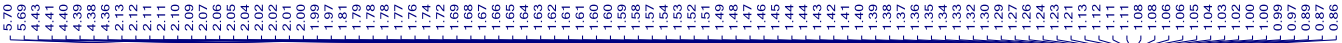<smiles>CCCC1C[C@@H]2CCCC[C@H]2[C@H]1NC(=O)OC</smiles>

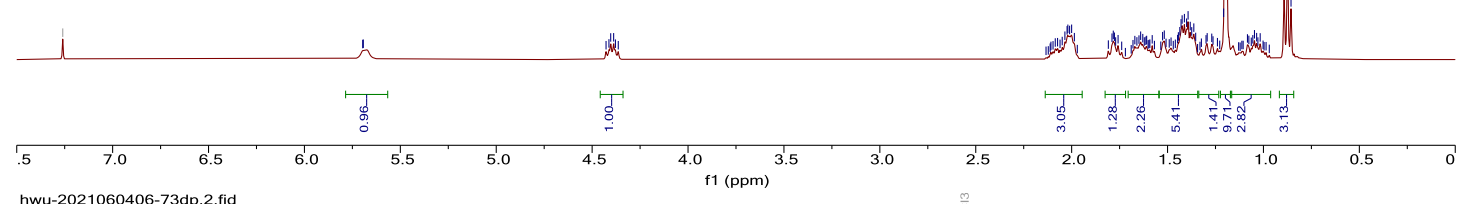
hwu-2021060406-73dp.2.fid

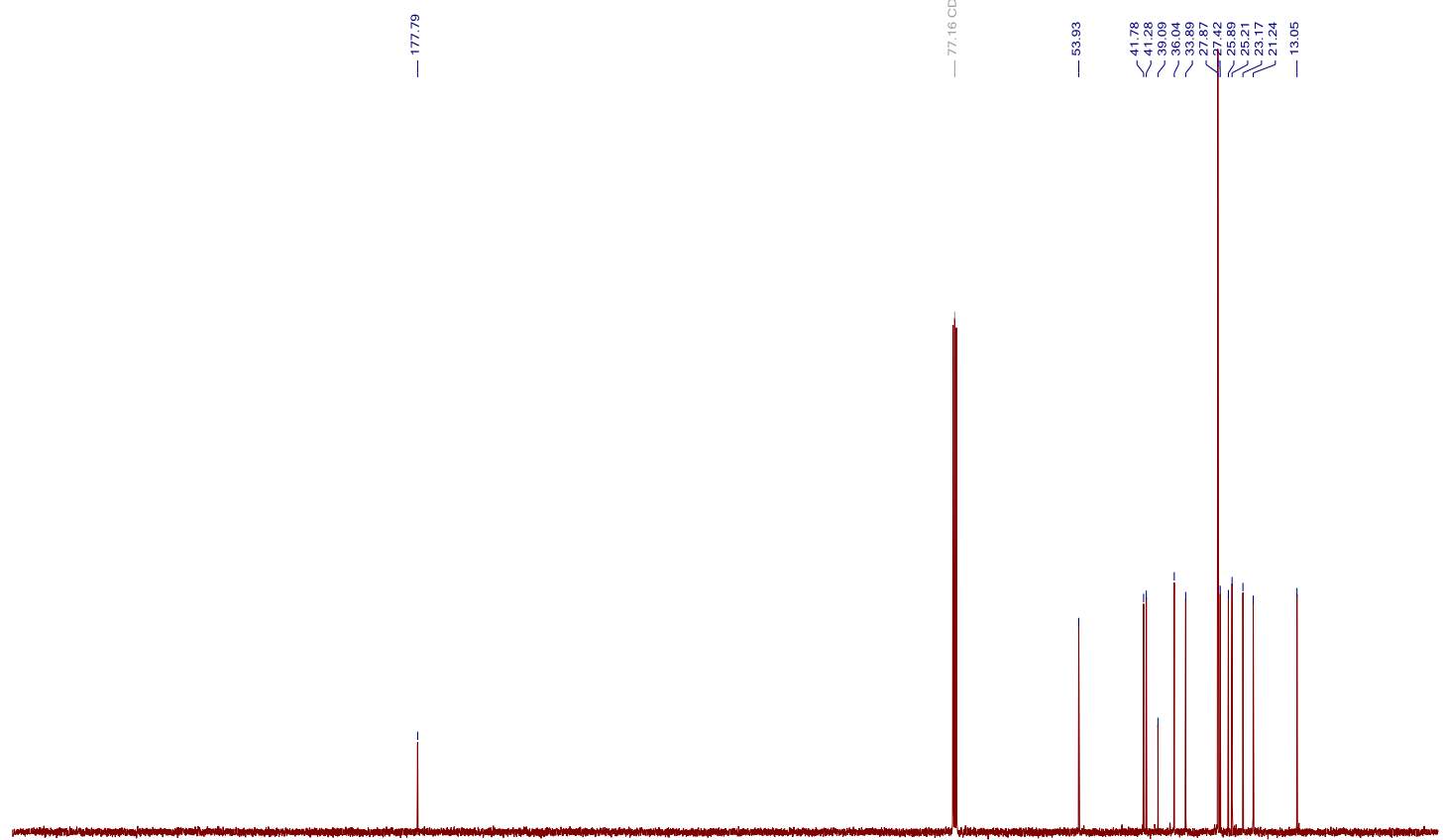

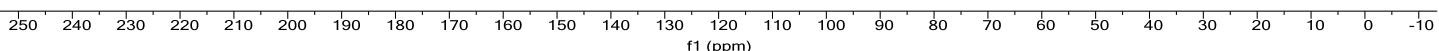


fiwu-210616-206-84bp. 10.fid

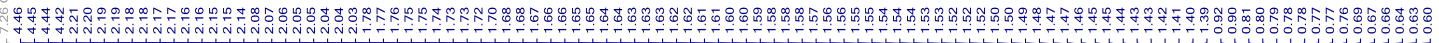<smiles>C[C@@H]1CC[C@@H]2[C@@H](CC[C@H]2NC(=O)OC(F)(F)F)C1</smiles>

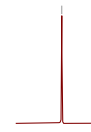

M
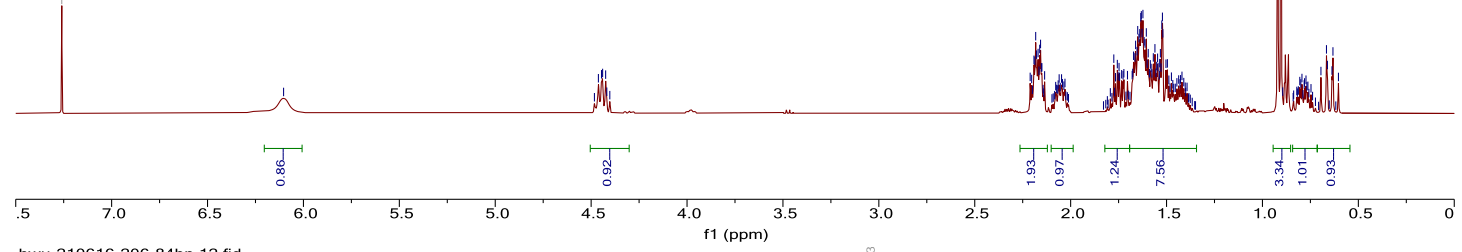

hwu-210616-206-84bp.12.fid

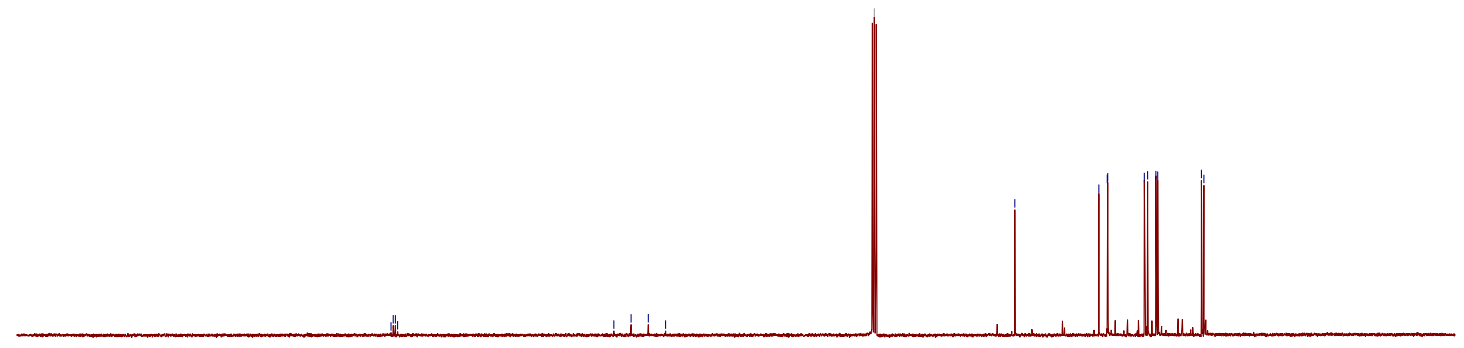

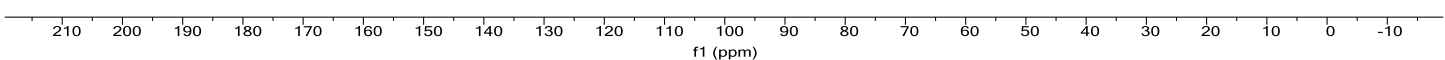


hwu-210616-206-84bp.11.fid

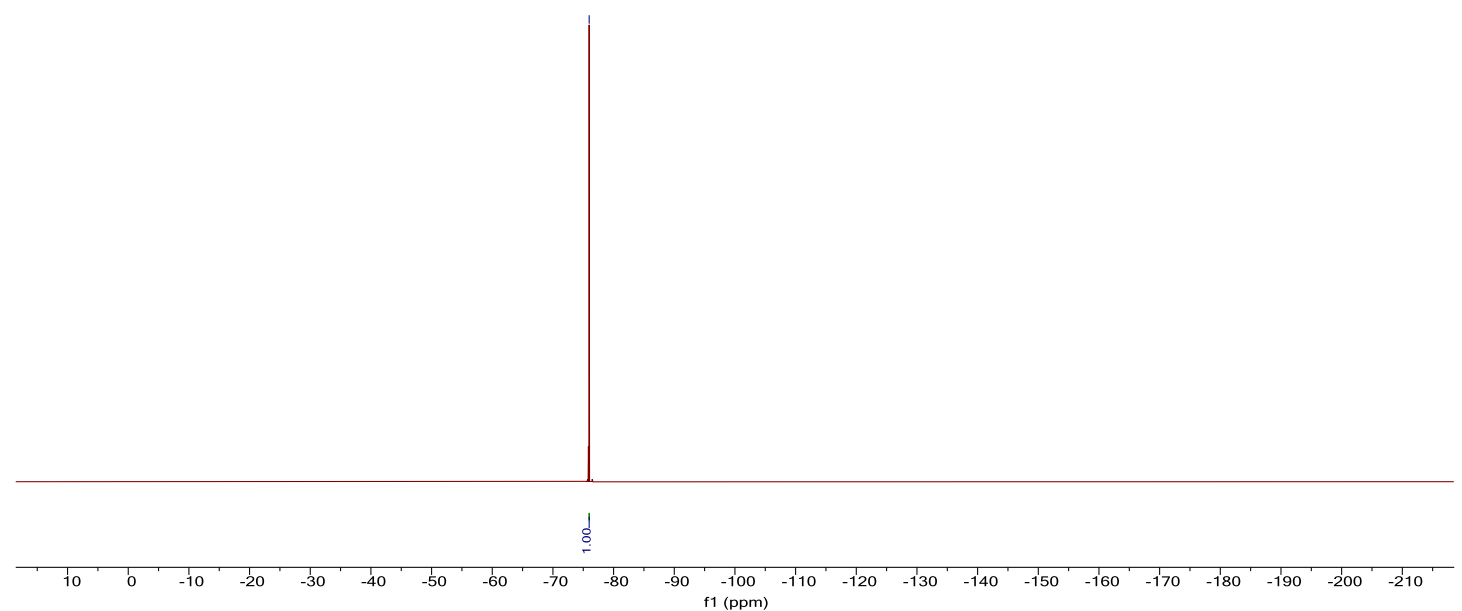


fiwu-210611-206-79bp.10.fid

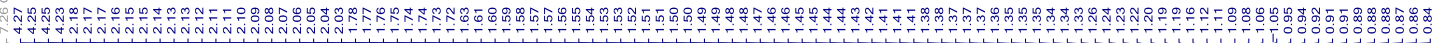<smiles>CN[C@H]1CC[C@H]2[C@@H](C)CCC[C@@H]12</smiles>
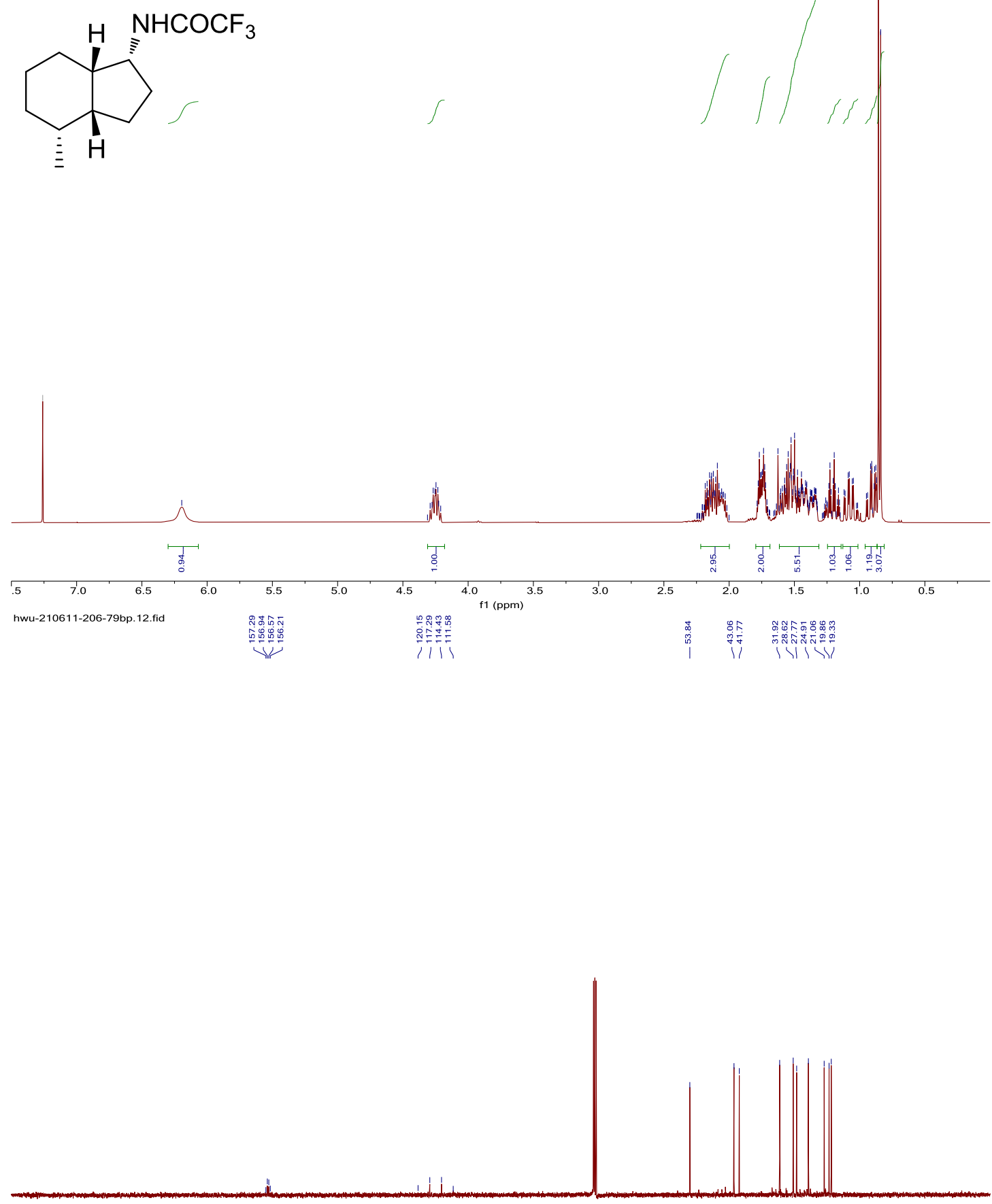

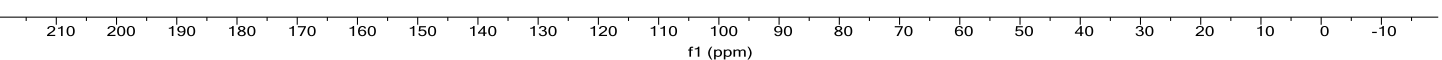




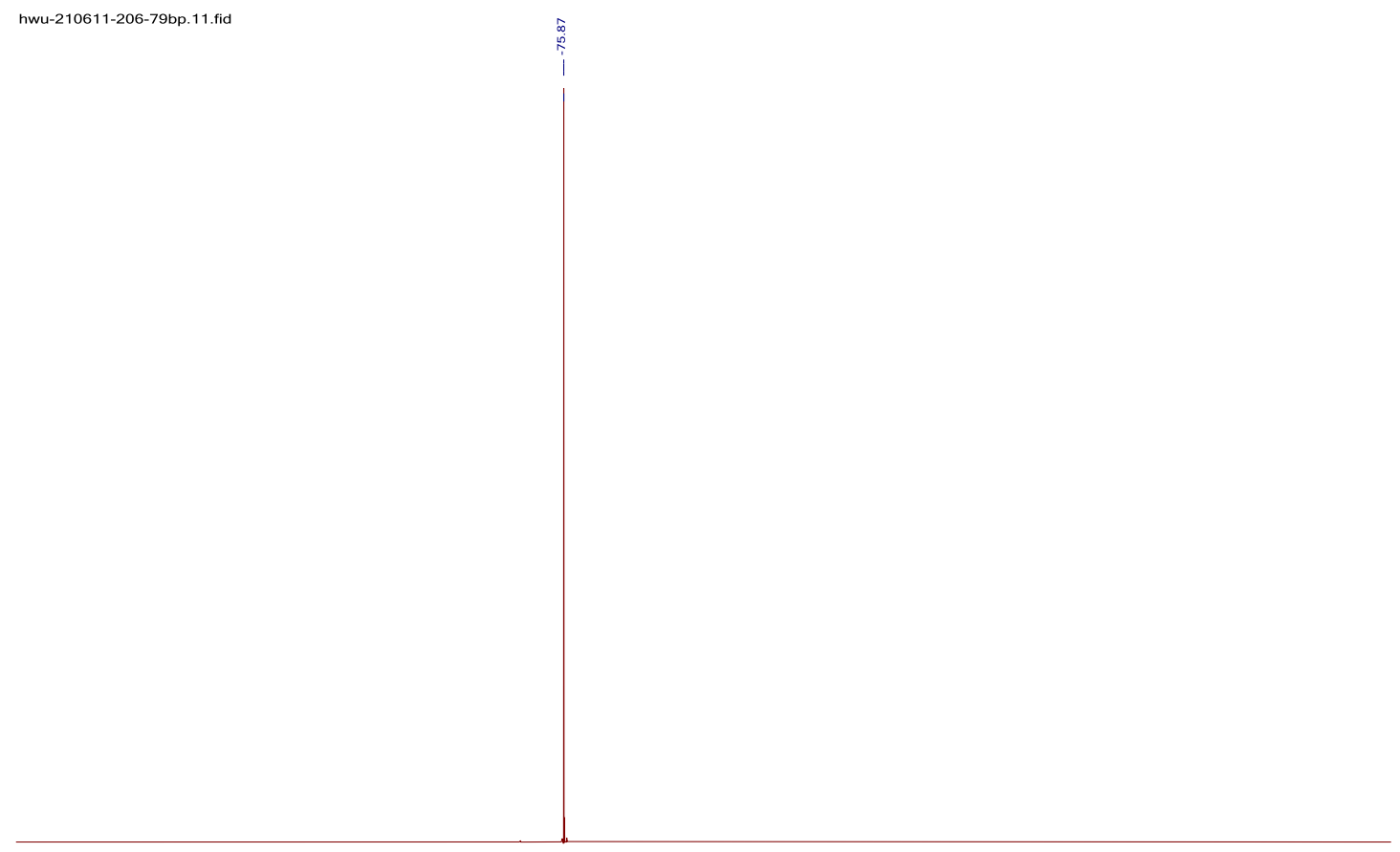

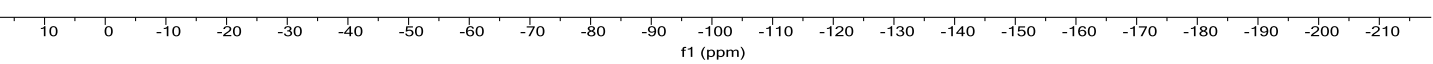


hwu-210616-206-84cp. 10; fid
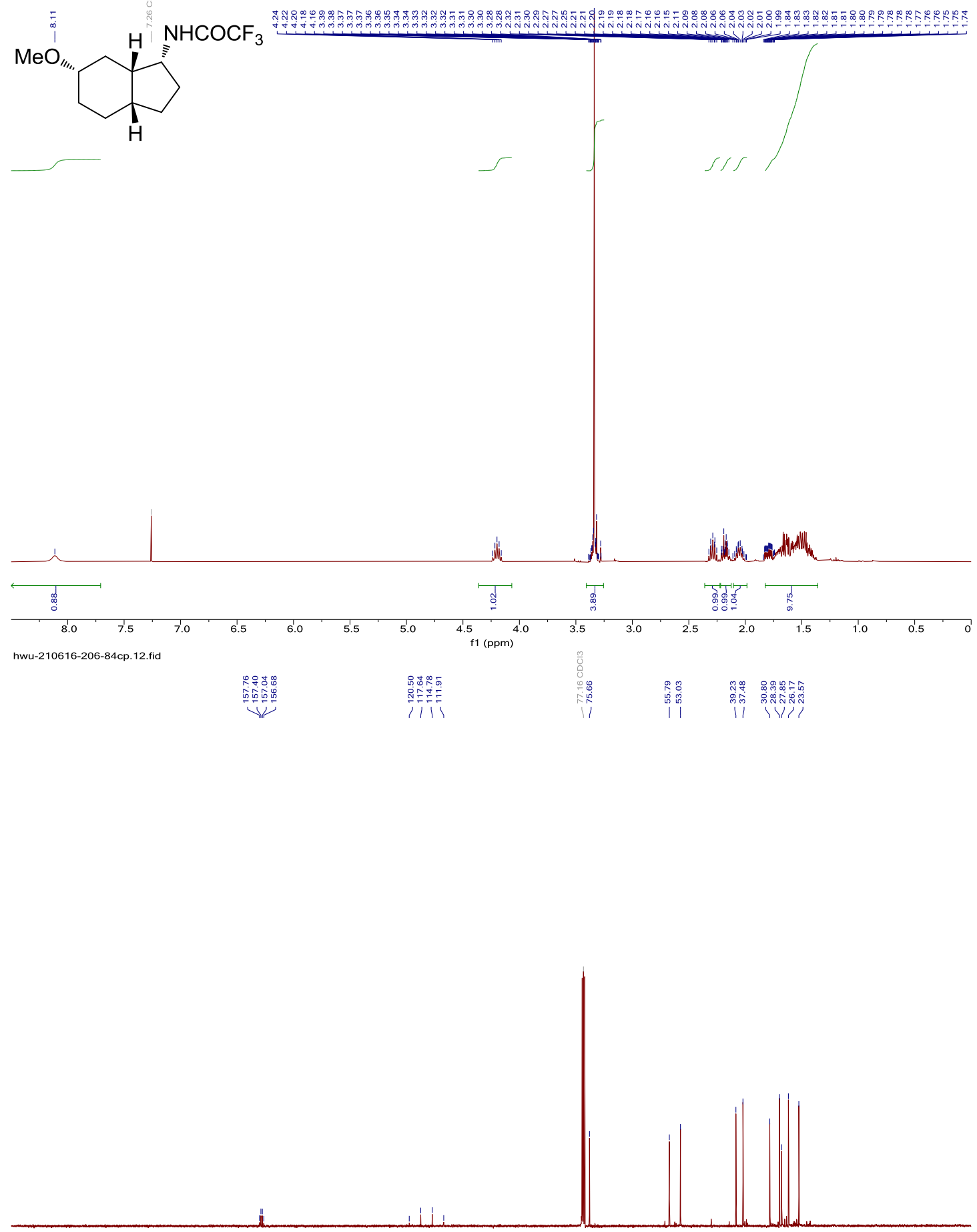

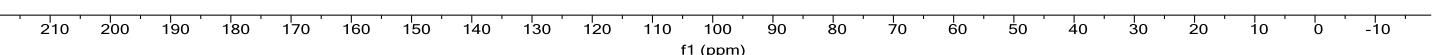




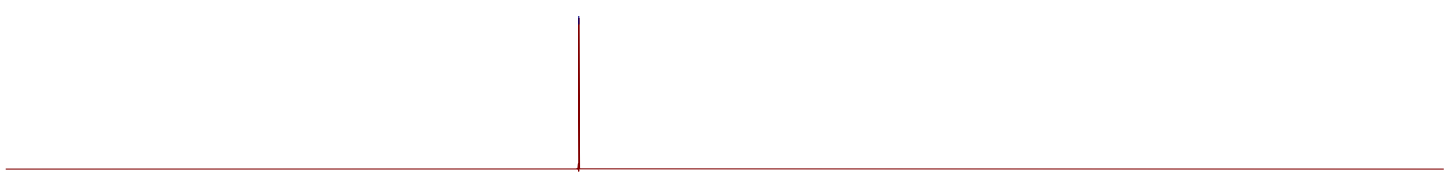

\begin{tabular}{llllllllllllllllllllllll}
10 & 0 & -10 & -20 & -30 & -40 & -50 & -60 & -70 & -80 & -90 & -100 & -110 & -120 & -130 & -140 & -150 & -160 & -170 & -180 & -190 & -200 & -210 \\
\hline
\end{tabular} 


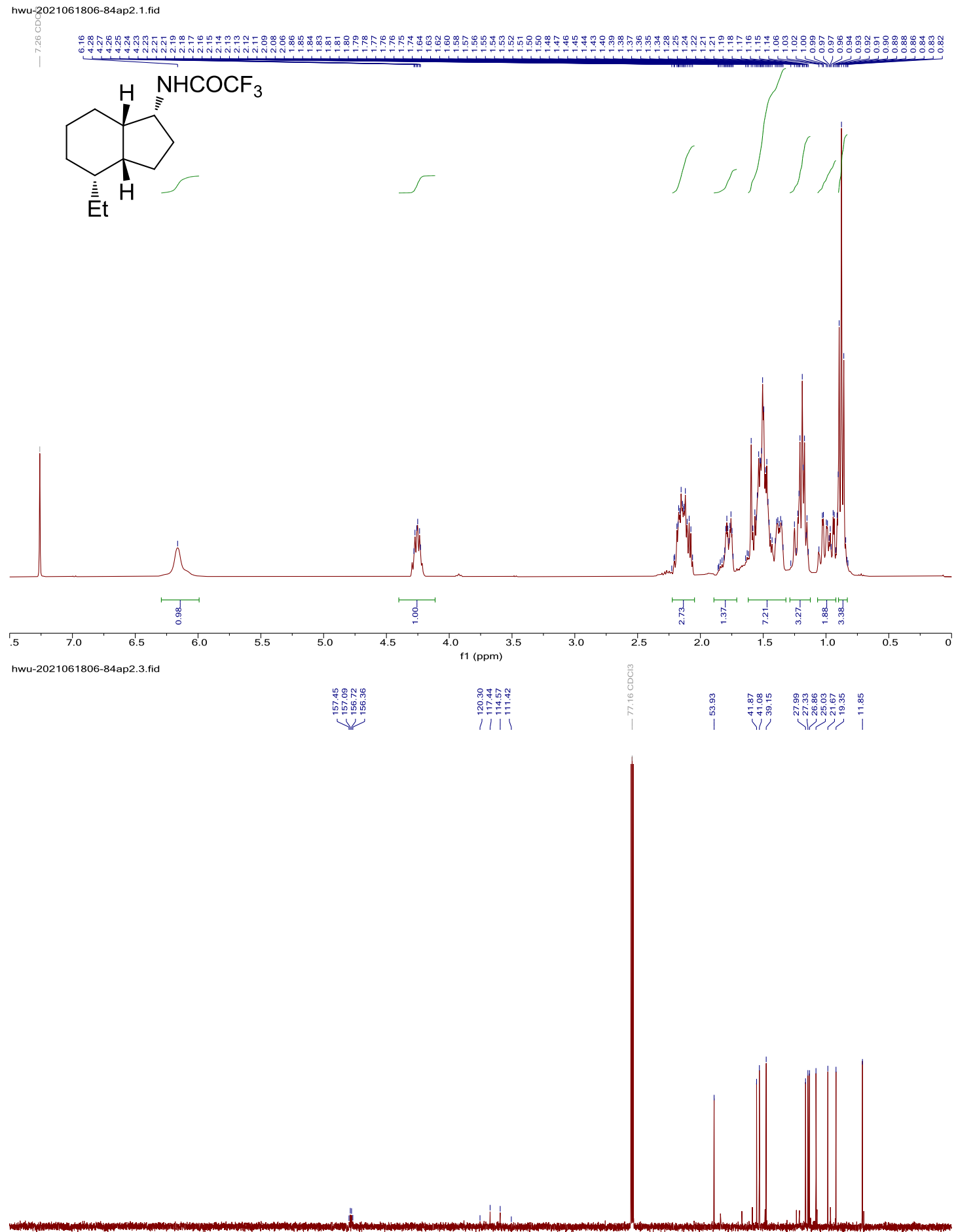

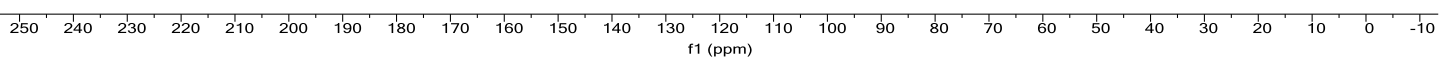




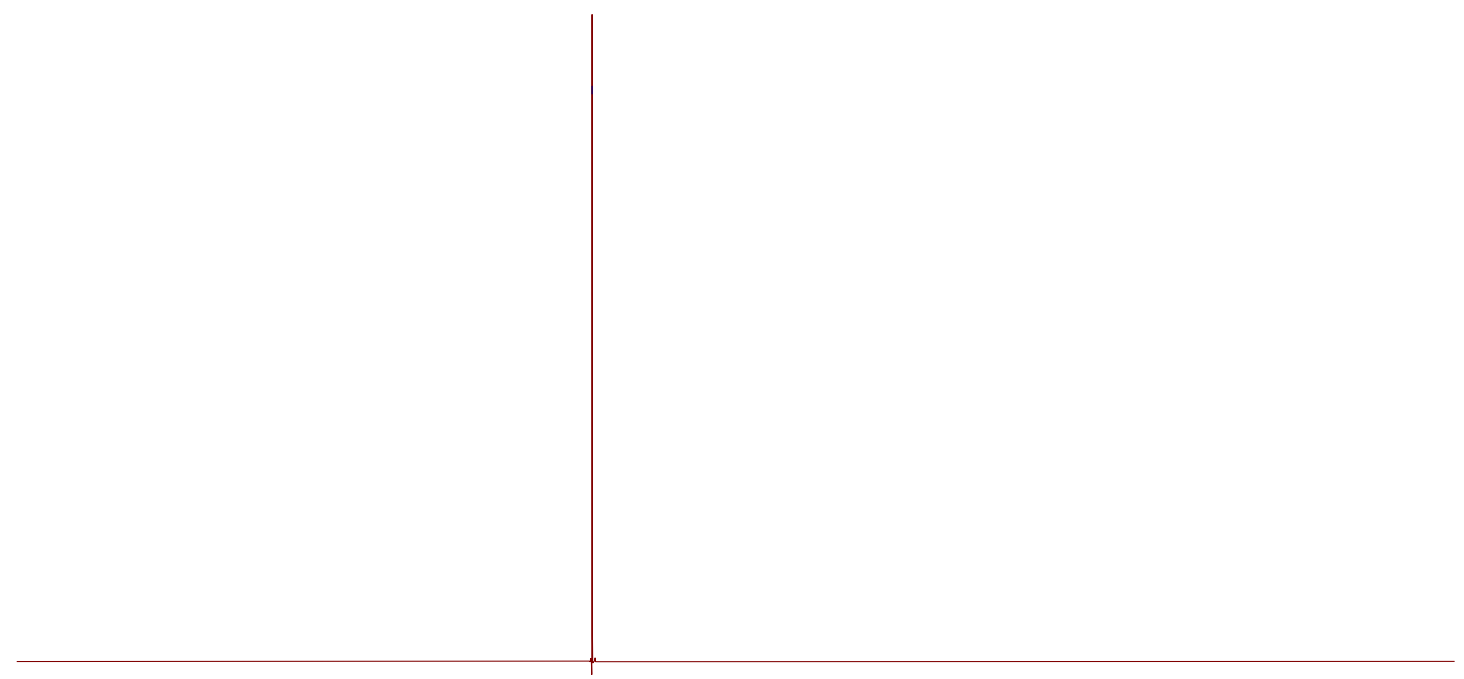

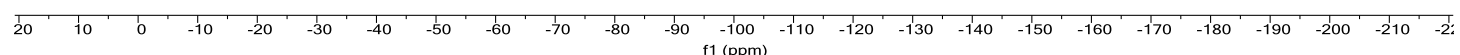




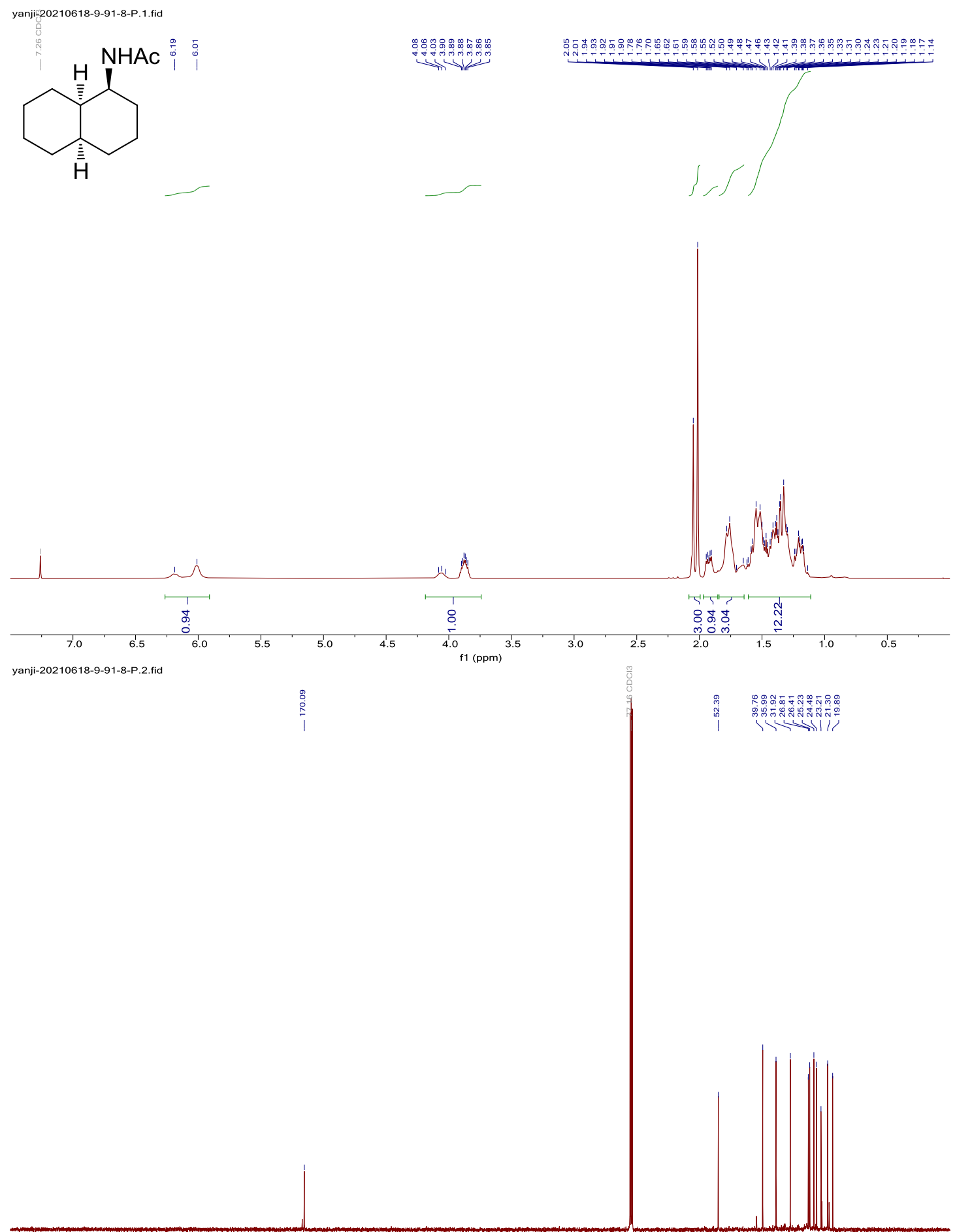

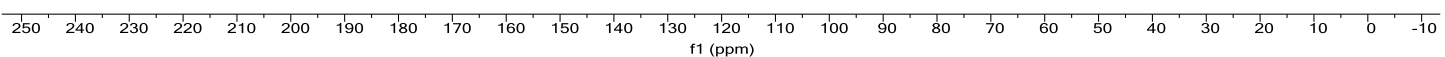




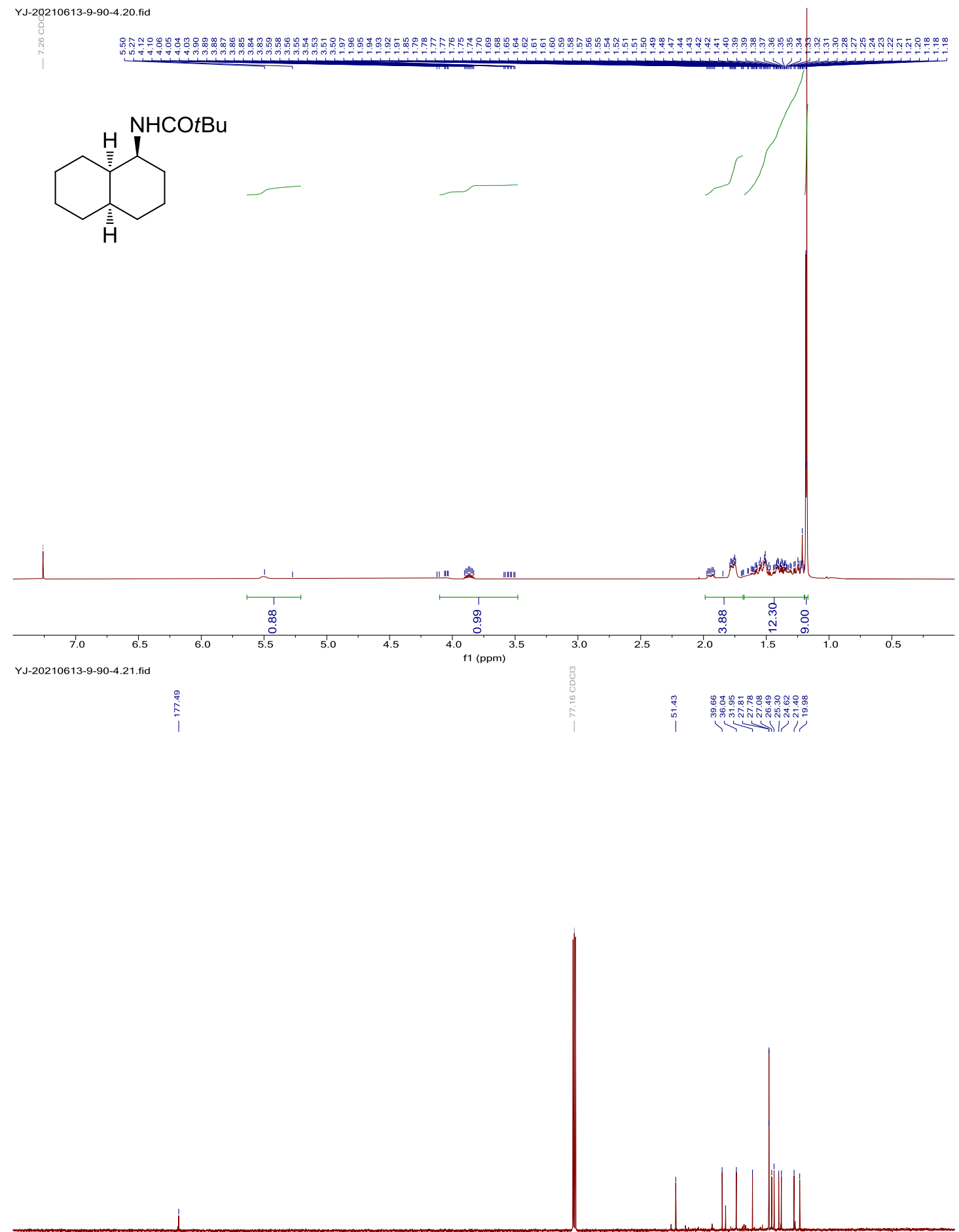

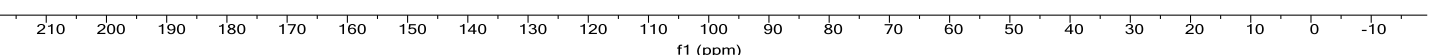



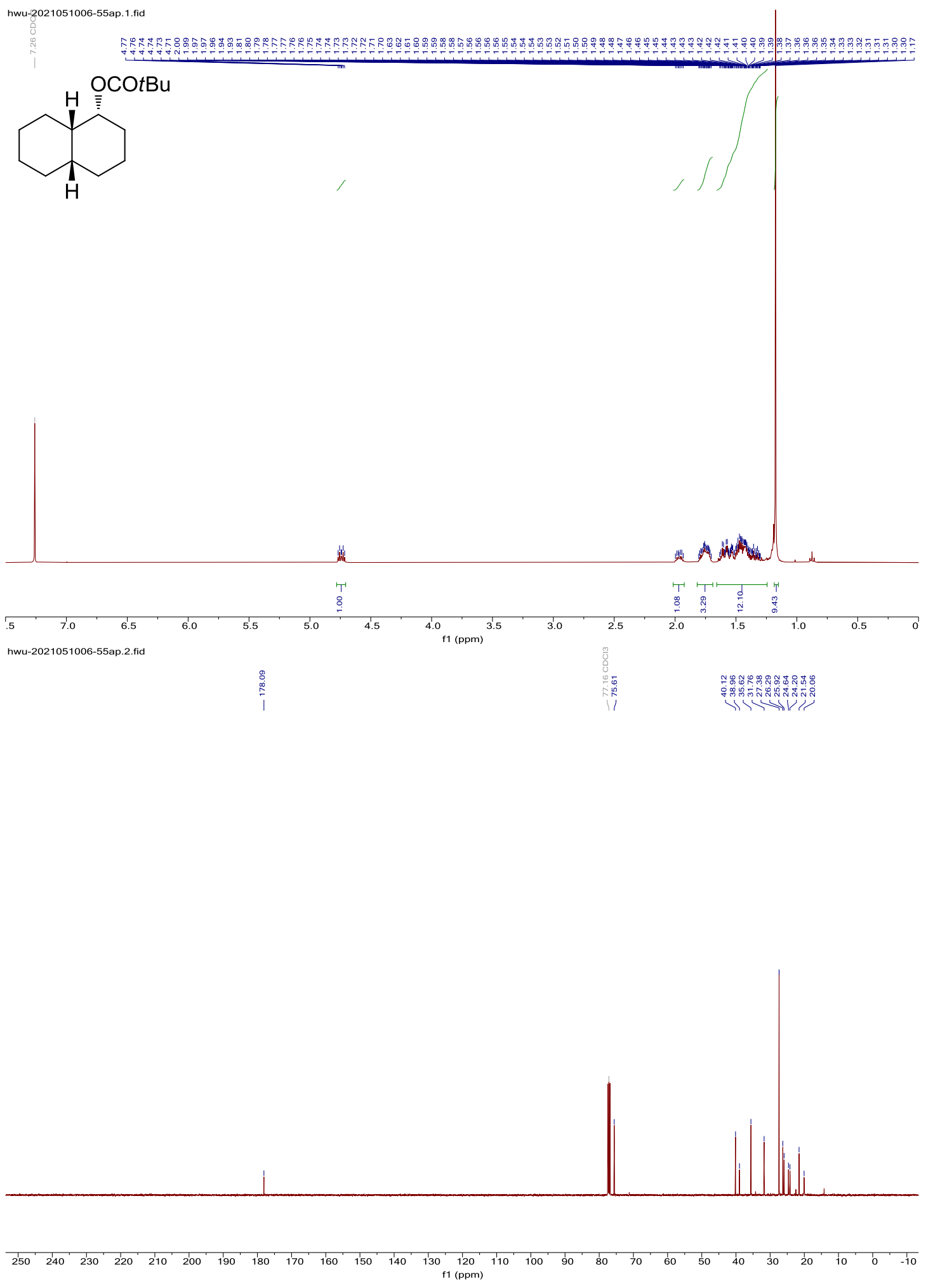

S103 


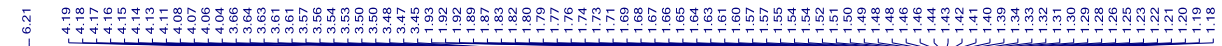<smiles>O=C(F)N[C@H]1CCO[C@H]2CCCC[C@H]12</smiles>
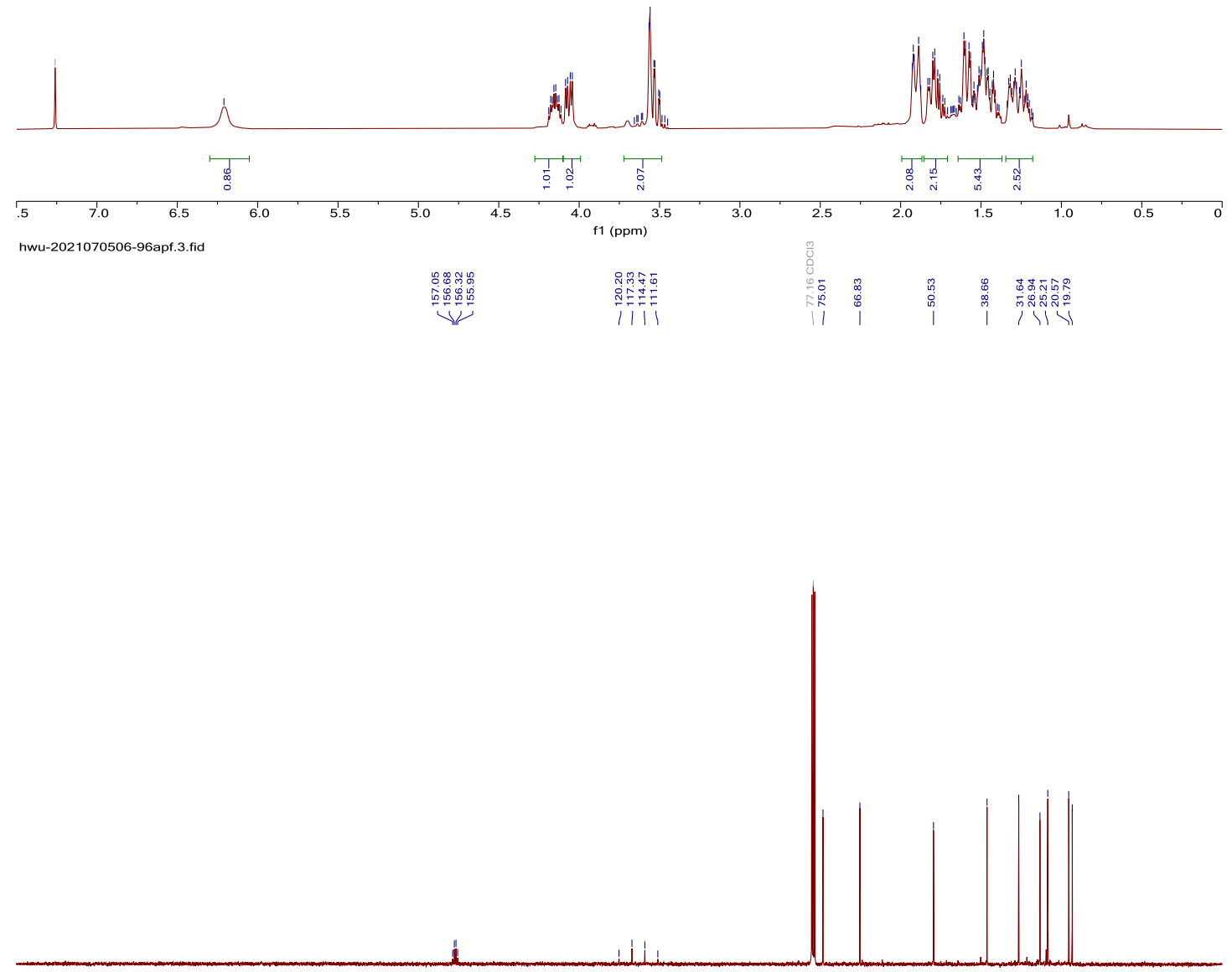

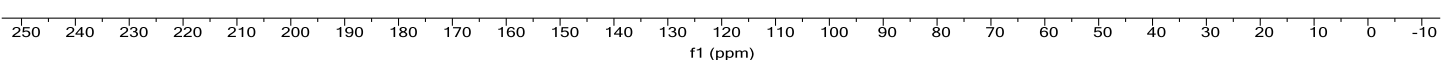




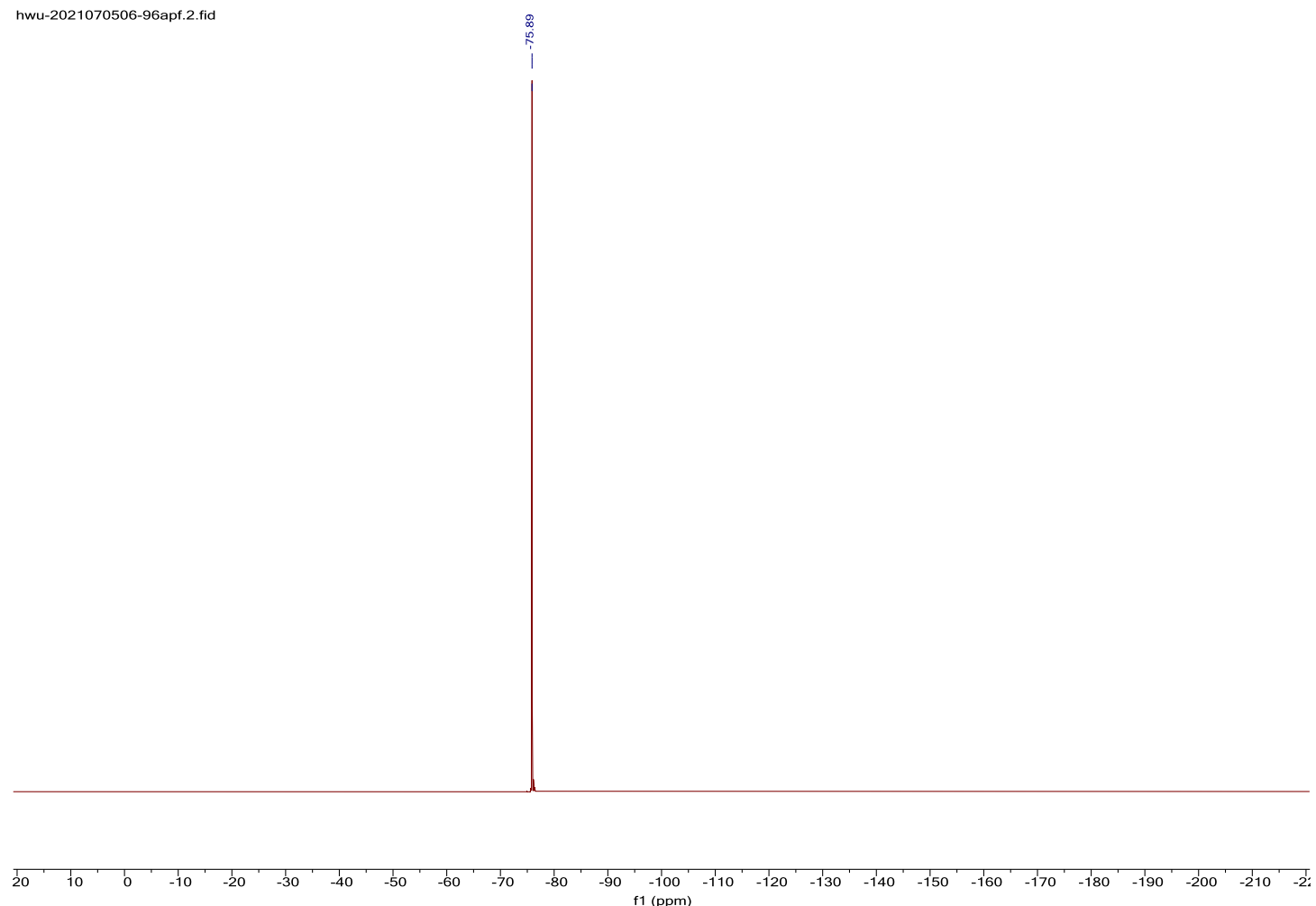


fiwu-210613-206-83ap.10.fid

6.0.<smiles>CCCCO[C@H]1CCC[C@H]2[C@@H](C)CCC[C@H]12</smiles>
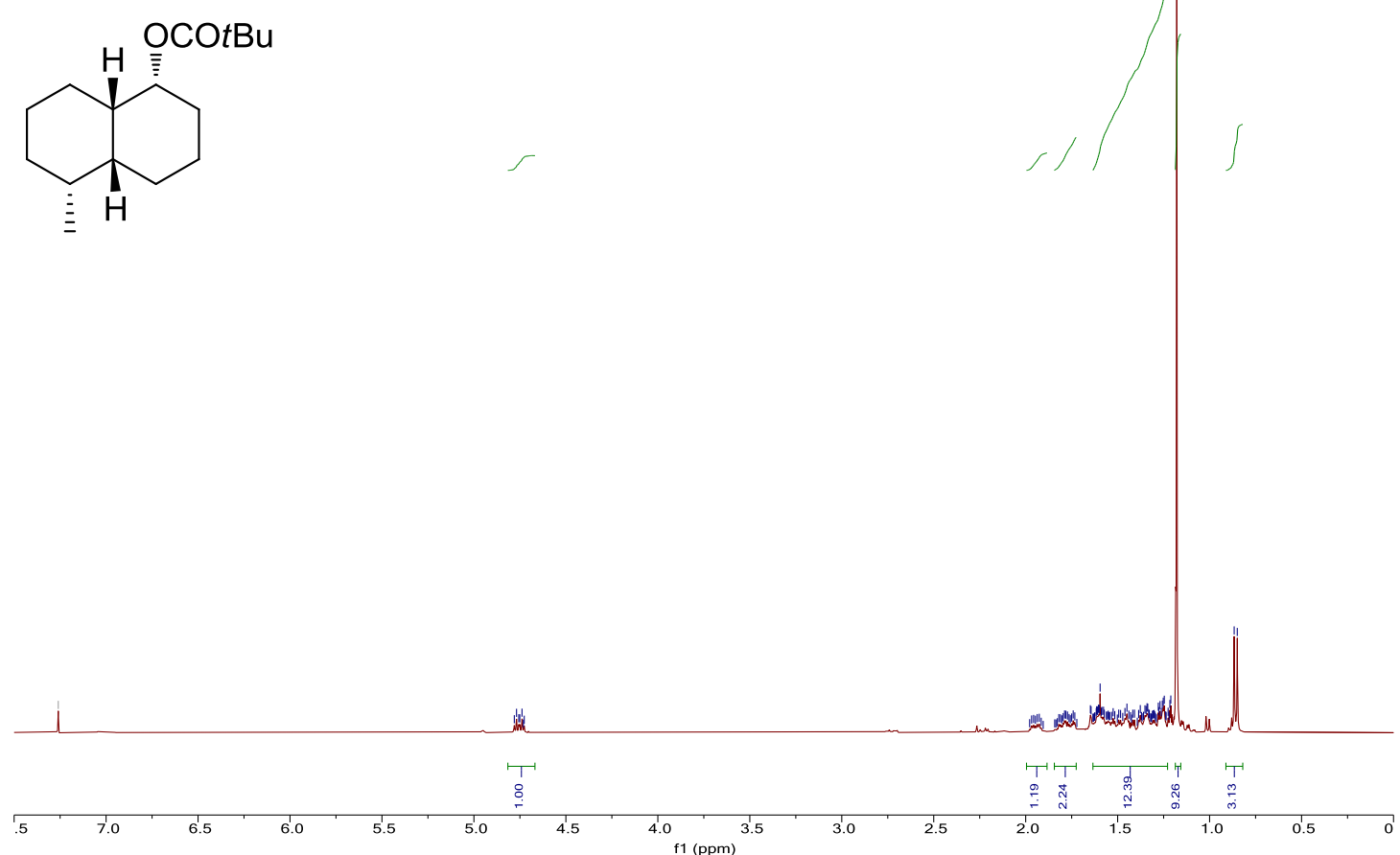

hwu-210613-206-83ap.11.fid $\stackrel{\circ}{\stackrel{\circ}{\infty}}$

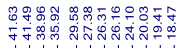

Vi, Tivi,

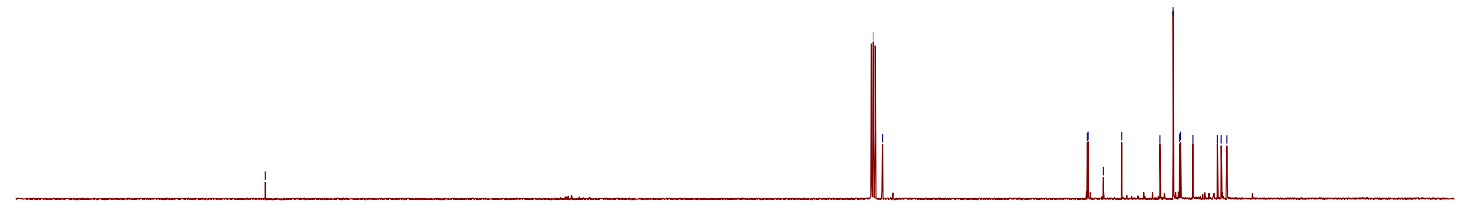

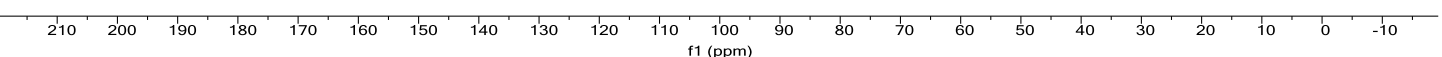


fुwu-210615-206-83cp2.10.fid

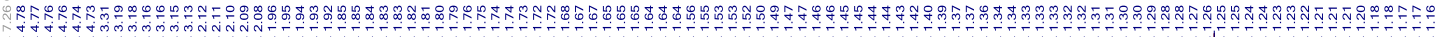<smiles>CCCCO[C@H]1CCC[C@@]2(C)[C@@H]1CCC[C@H]2OC(C)CC</smiles>

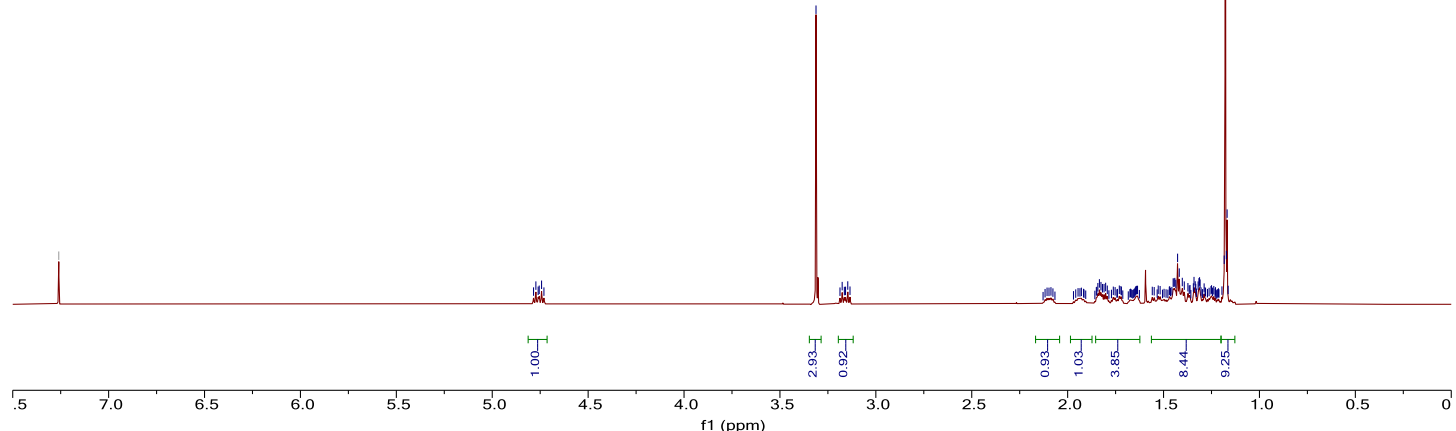
hwu-210615-206-83cp2.21.fid \begin{tabular}{l}
0 \\
0 \\
$\infty$ \\
0 \\
\hline
\end{tabular}

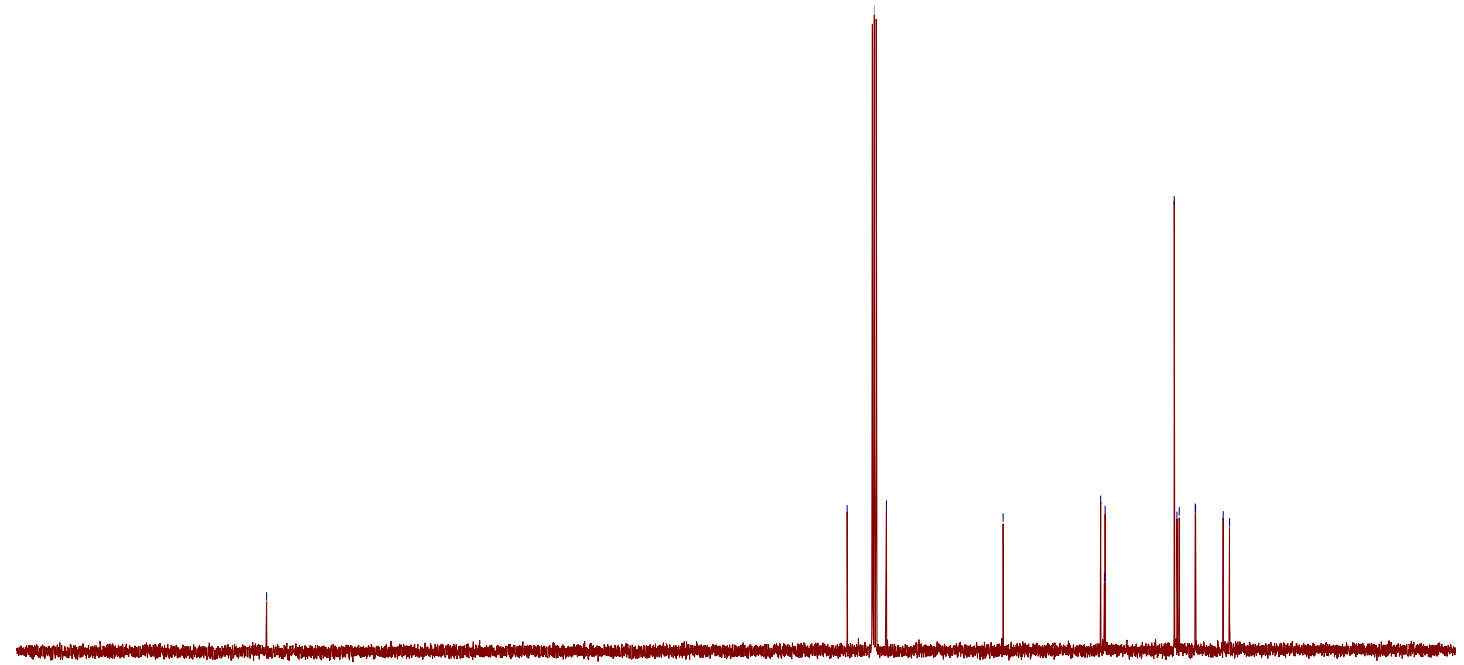

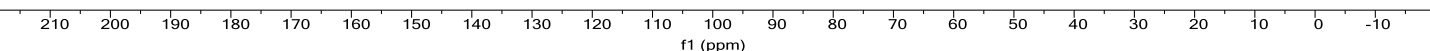


fiwu-210613-206-83bp.10.fid

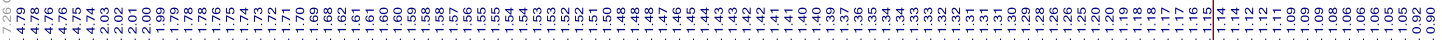<smiles>CCCO[C@H]1CCC[C@H]2CC[C@@H](C)C[C@@H]21</smiles>

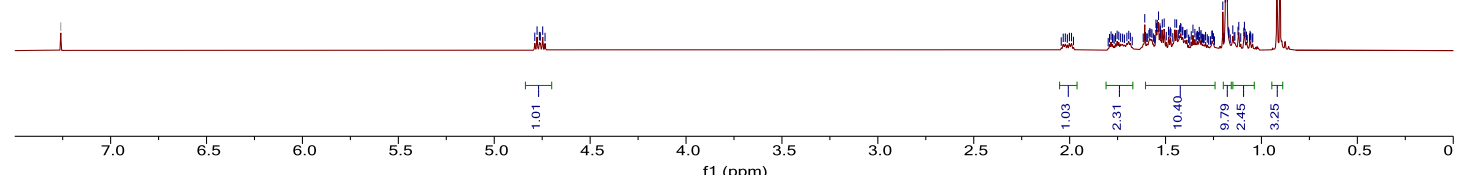

hwu-210613-206-83bp.11.fic

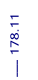

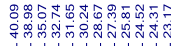

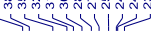

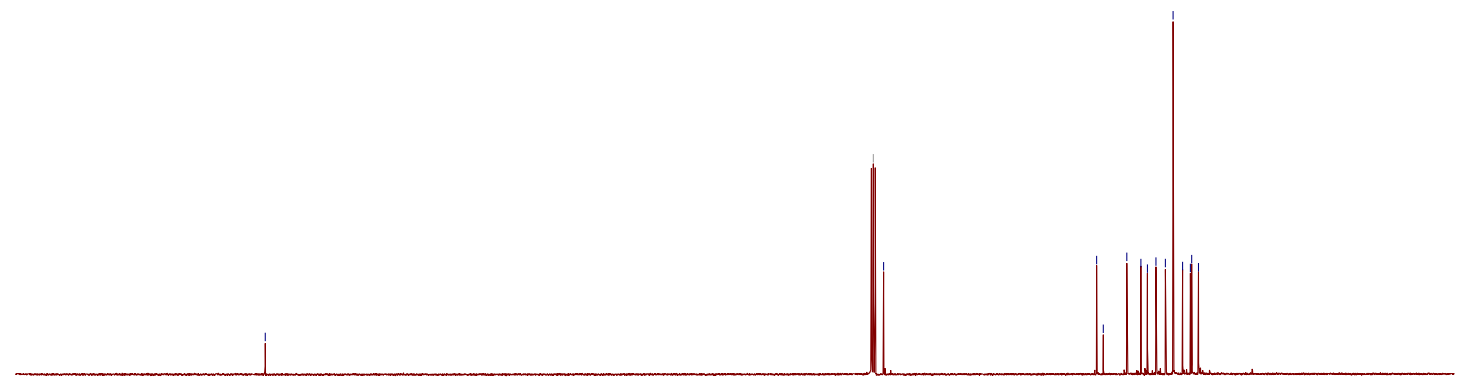

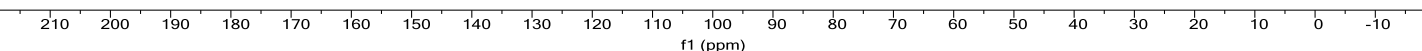


5은

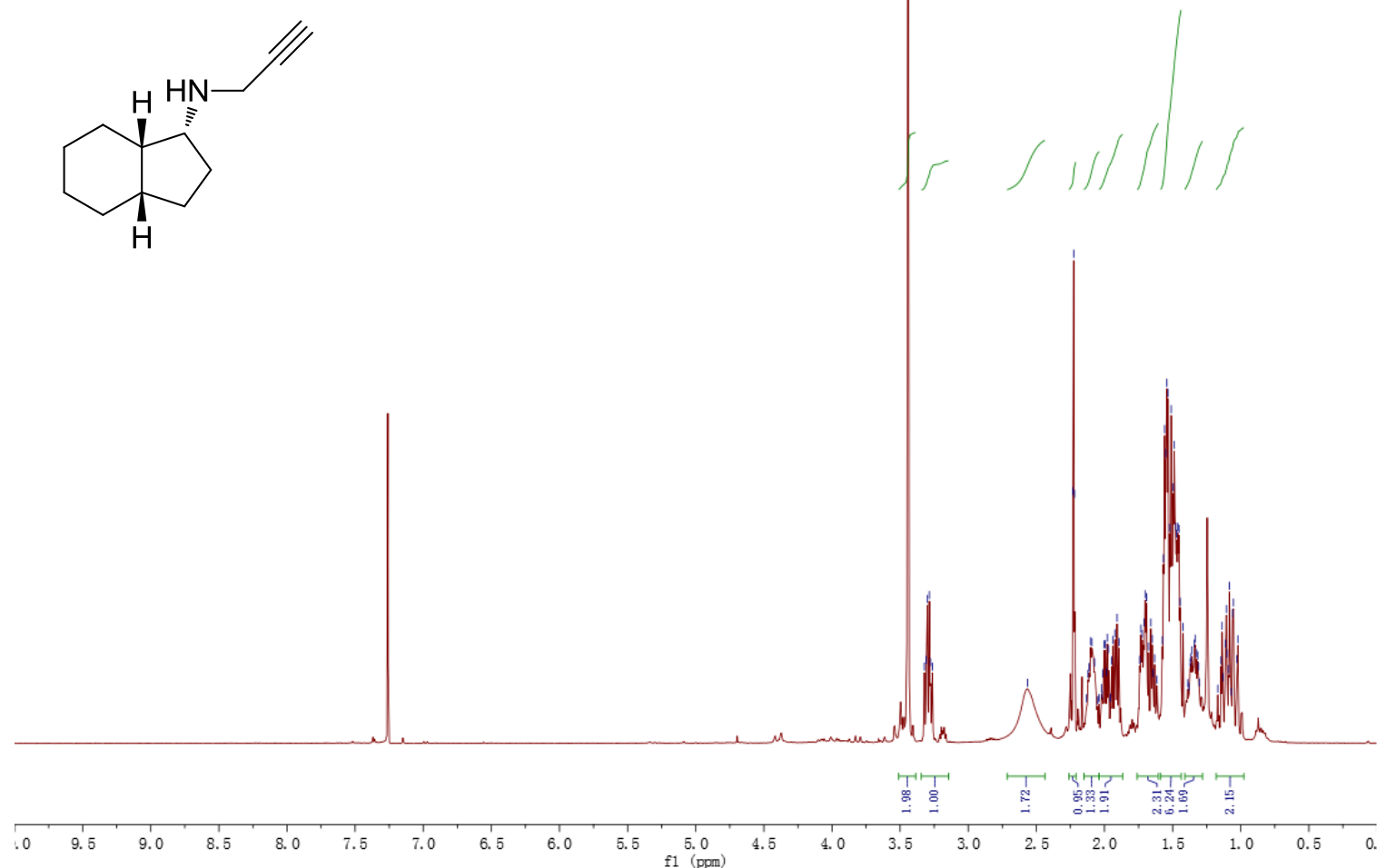

hwu-210713-206-100p. 11. fid

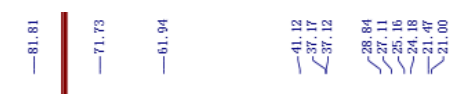
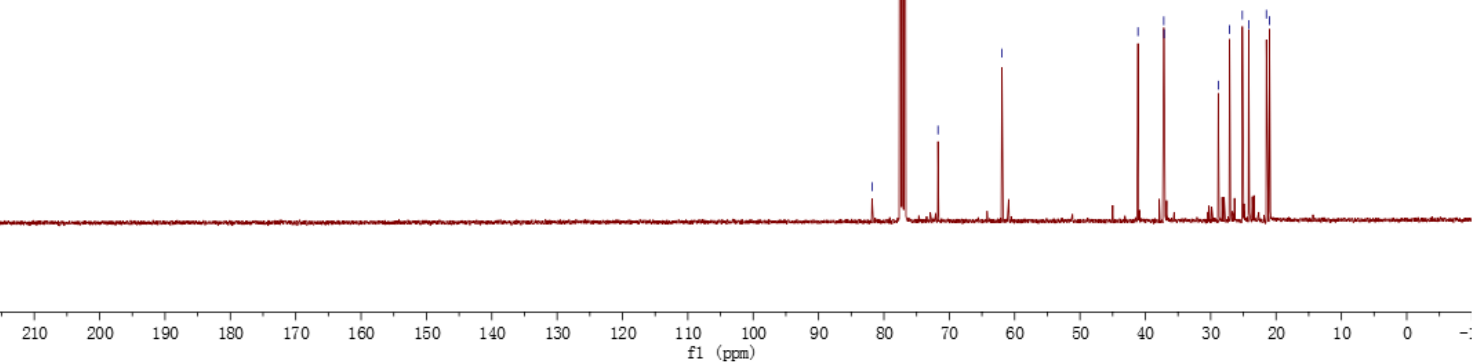
10. GC Chromatograms of hydrogenated products
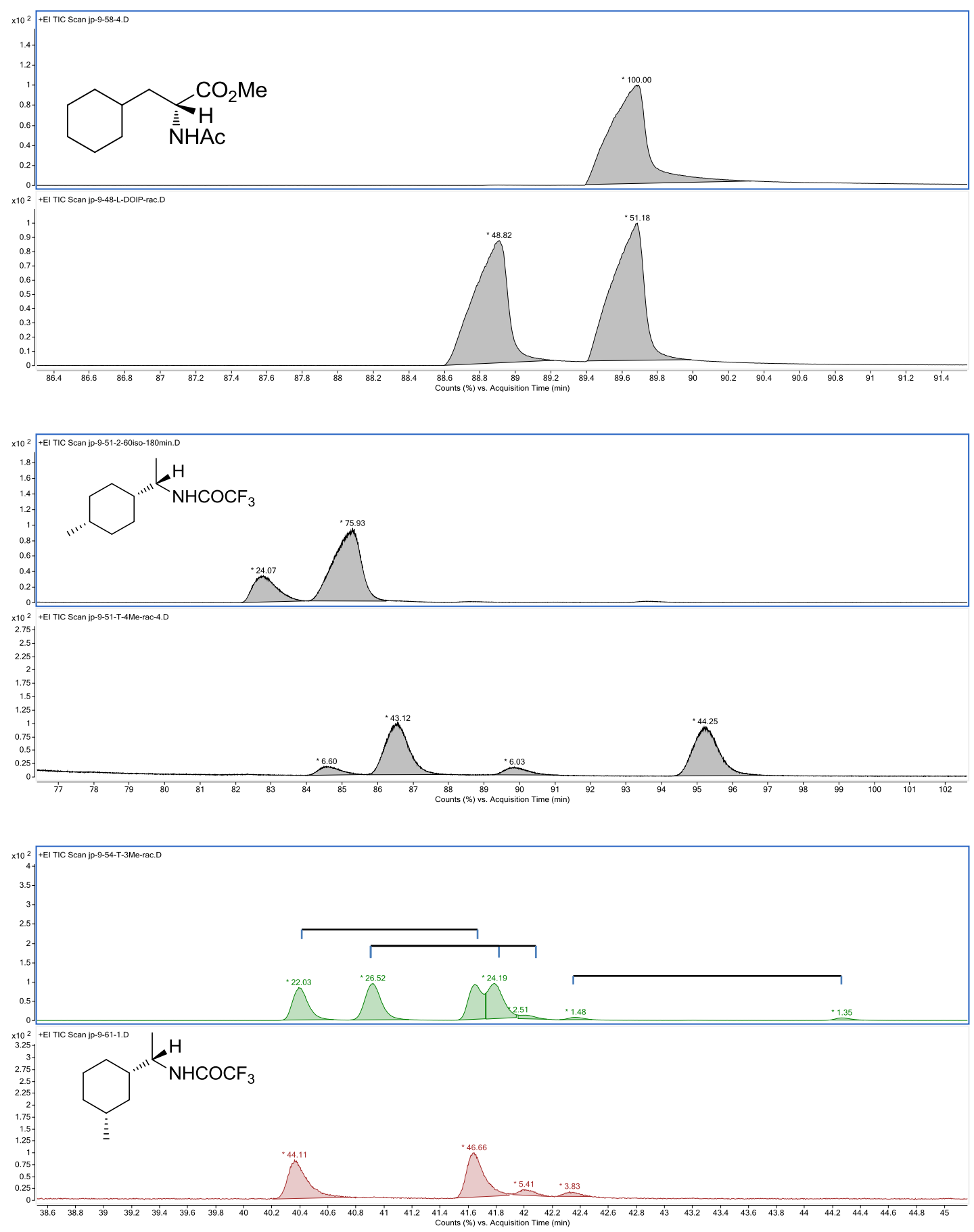

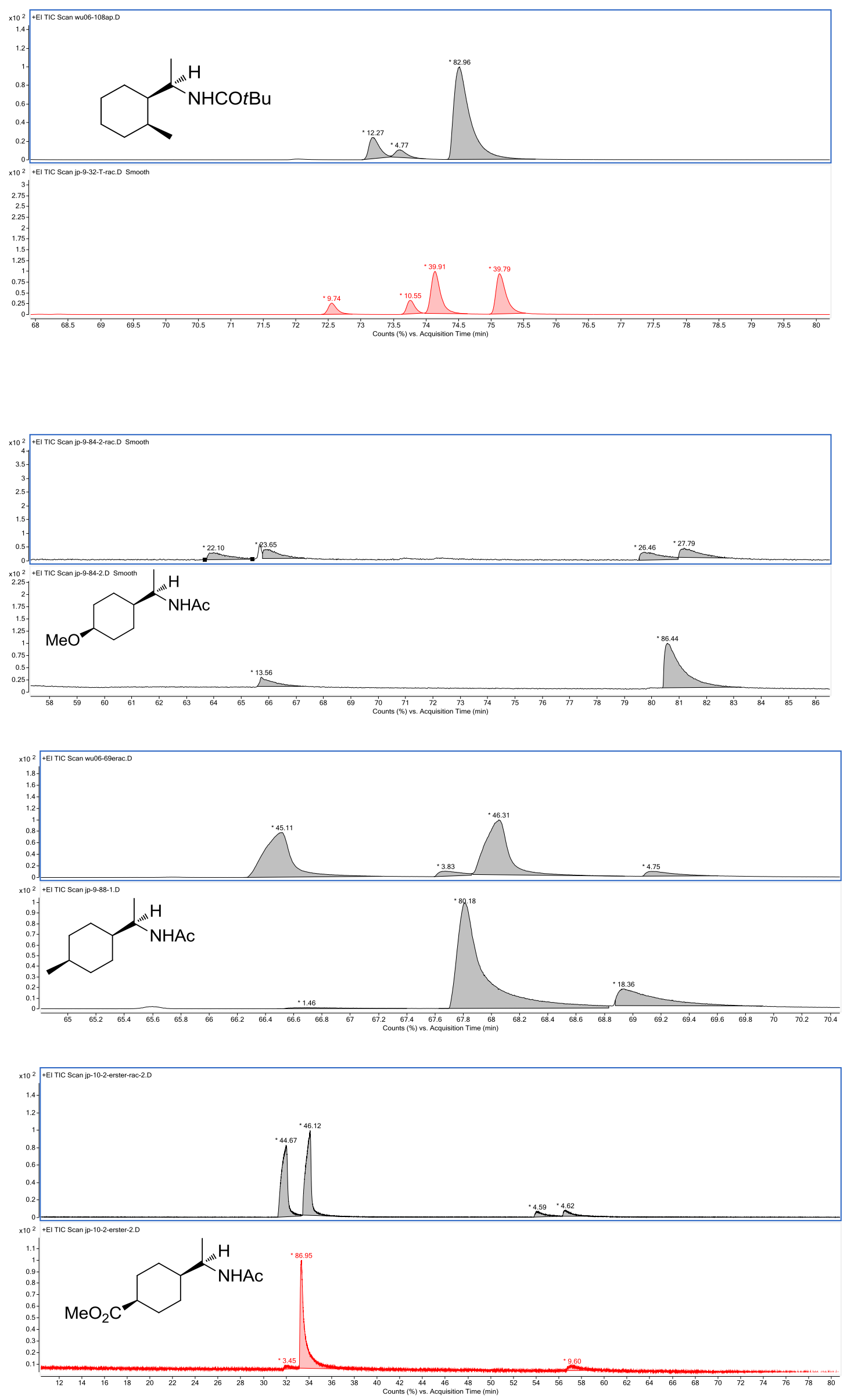

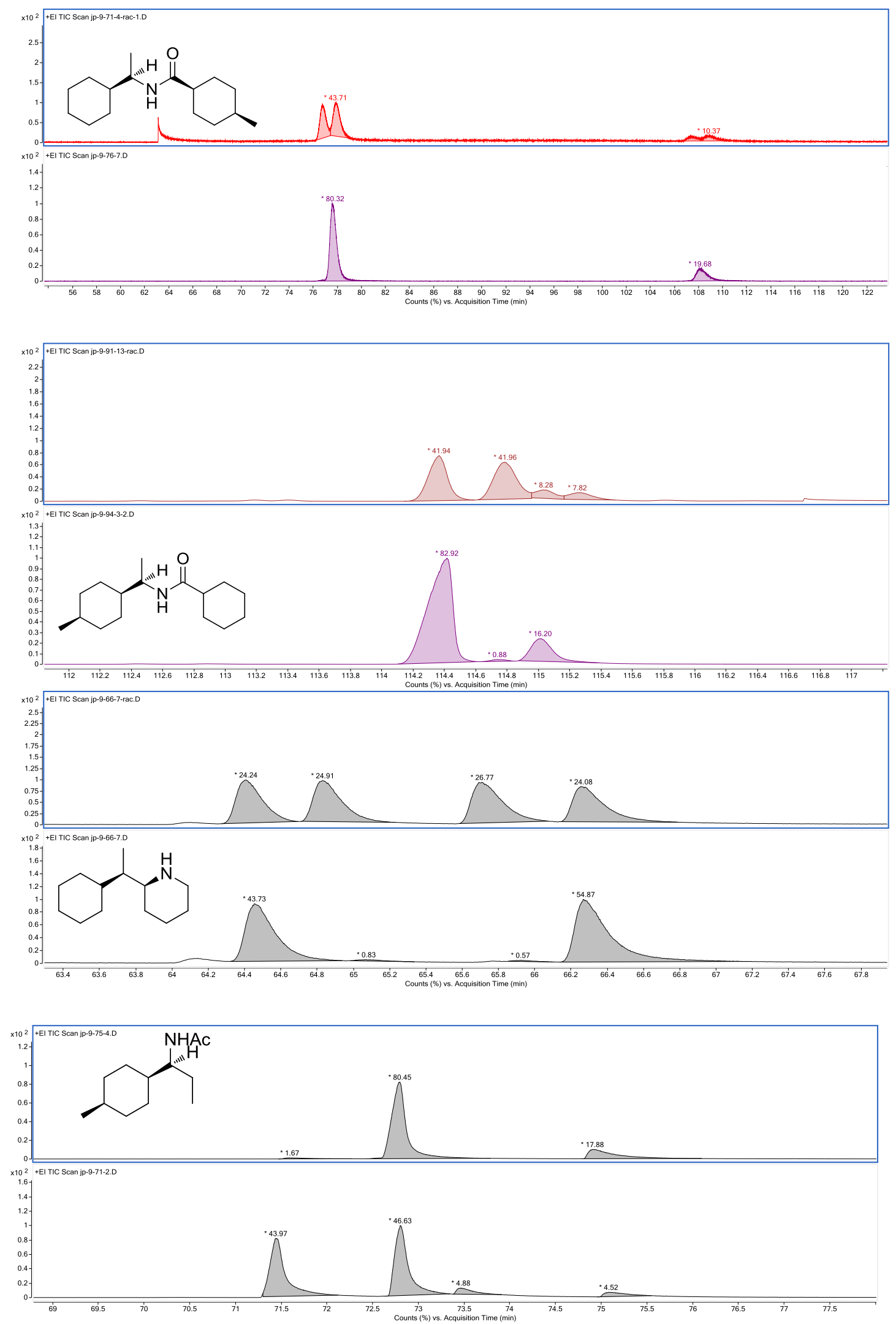

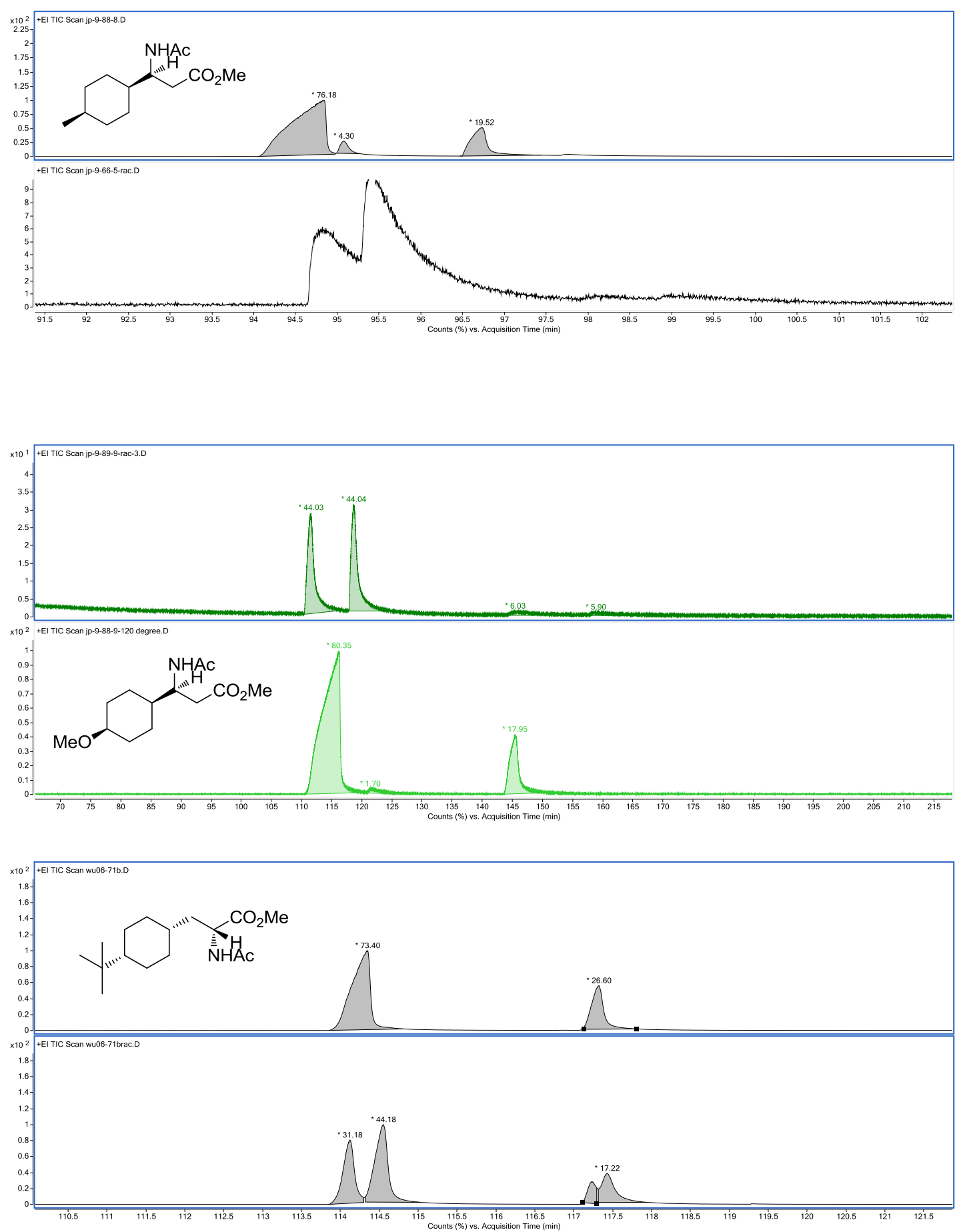

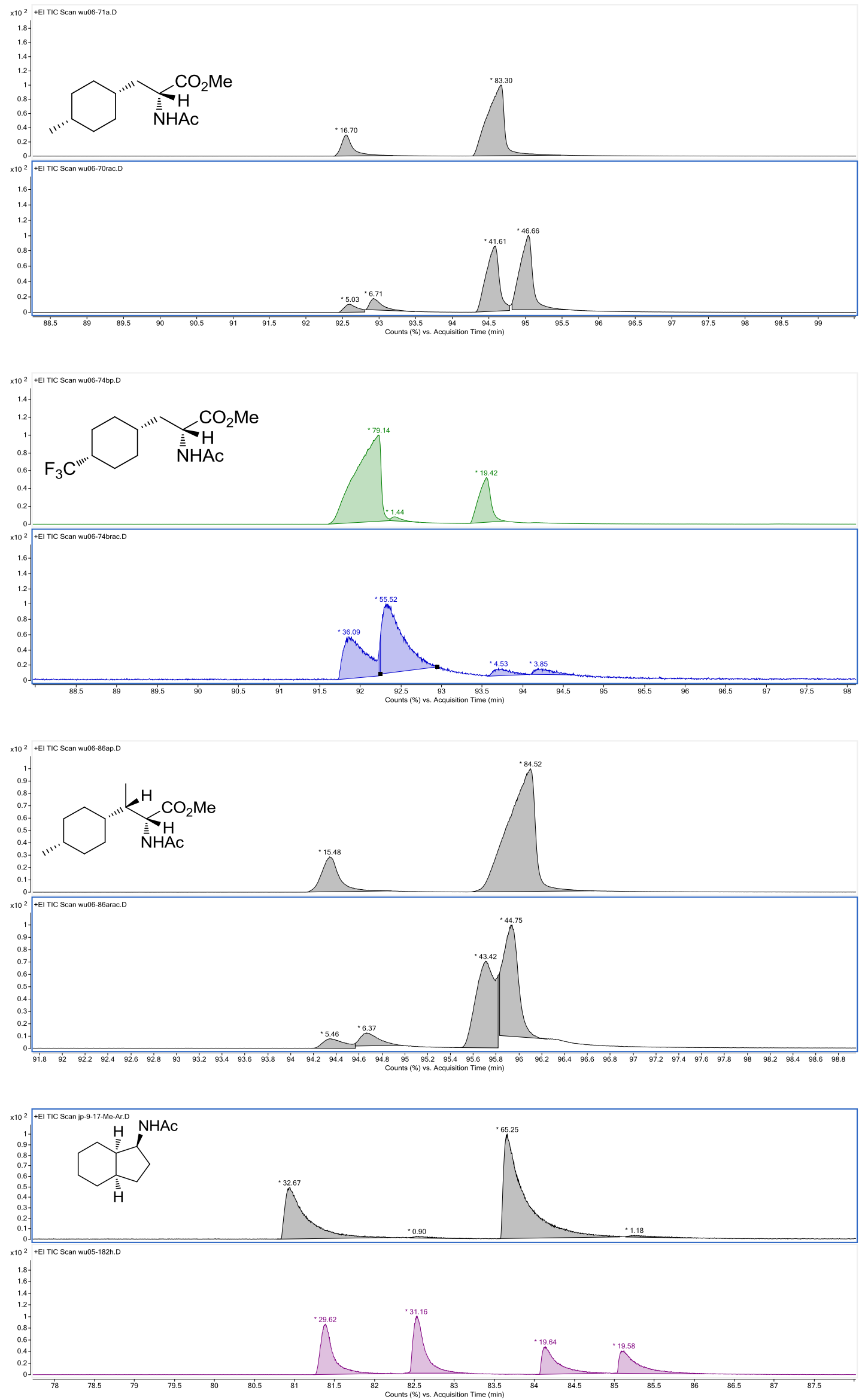

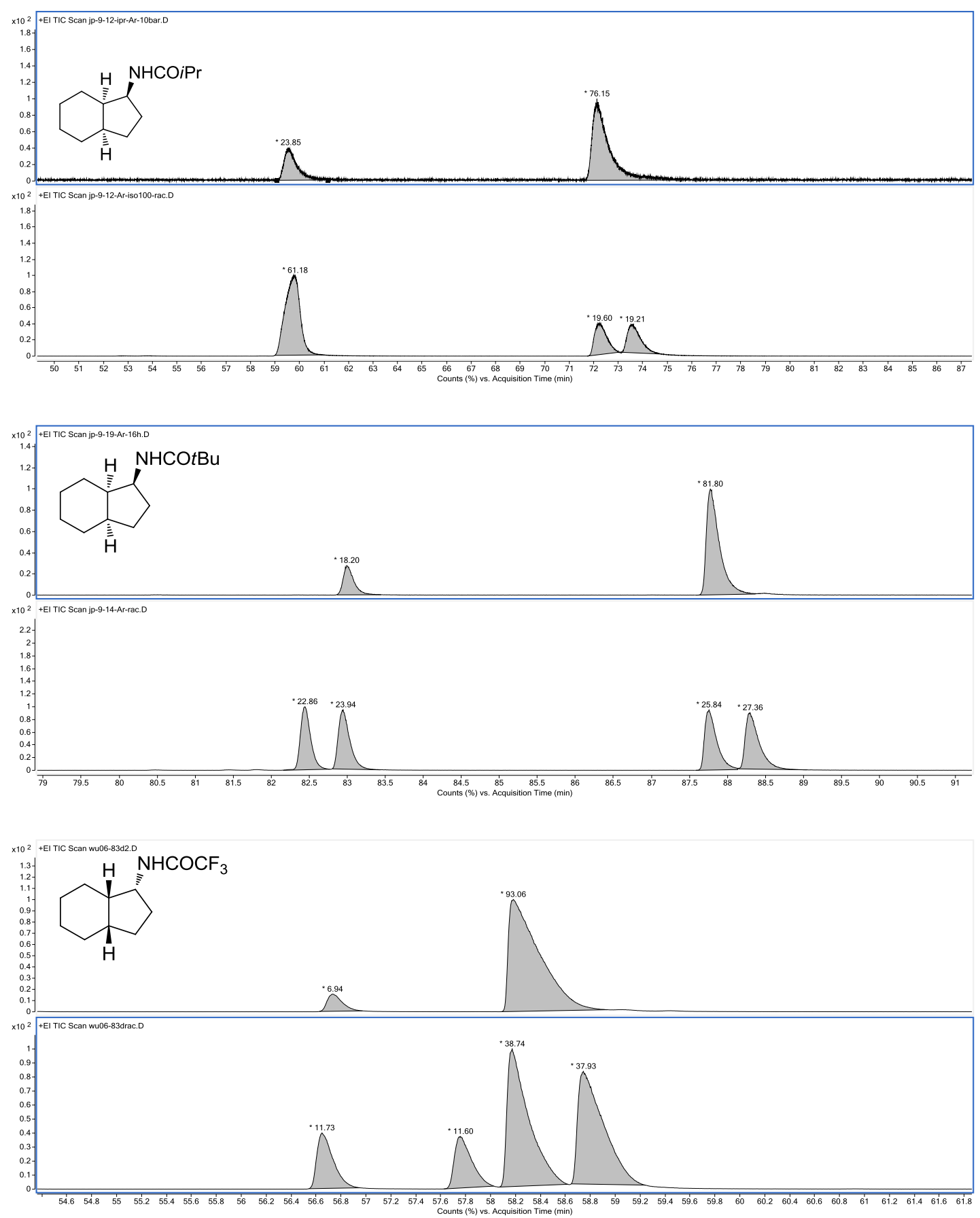

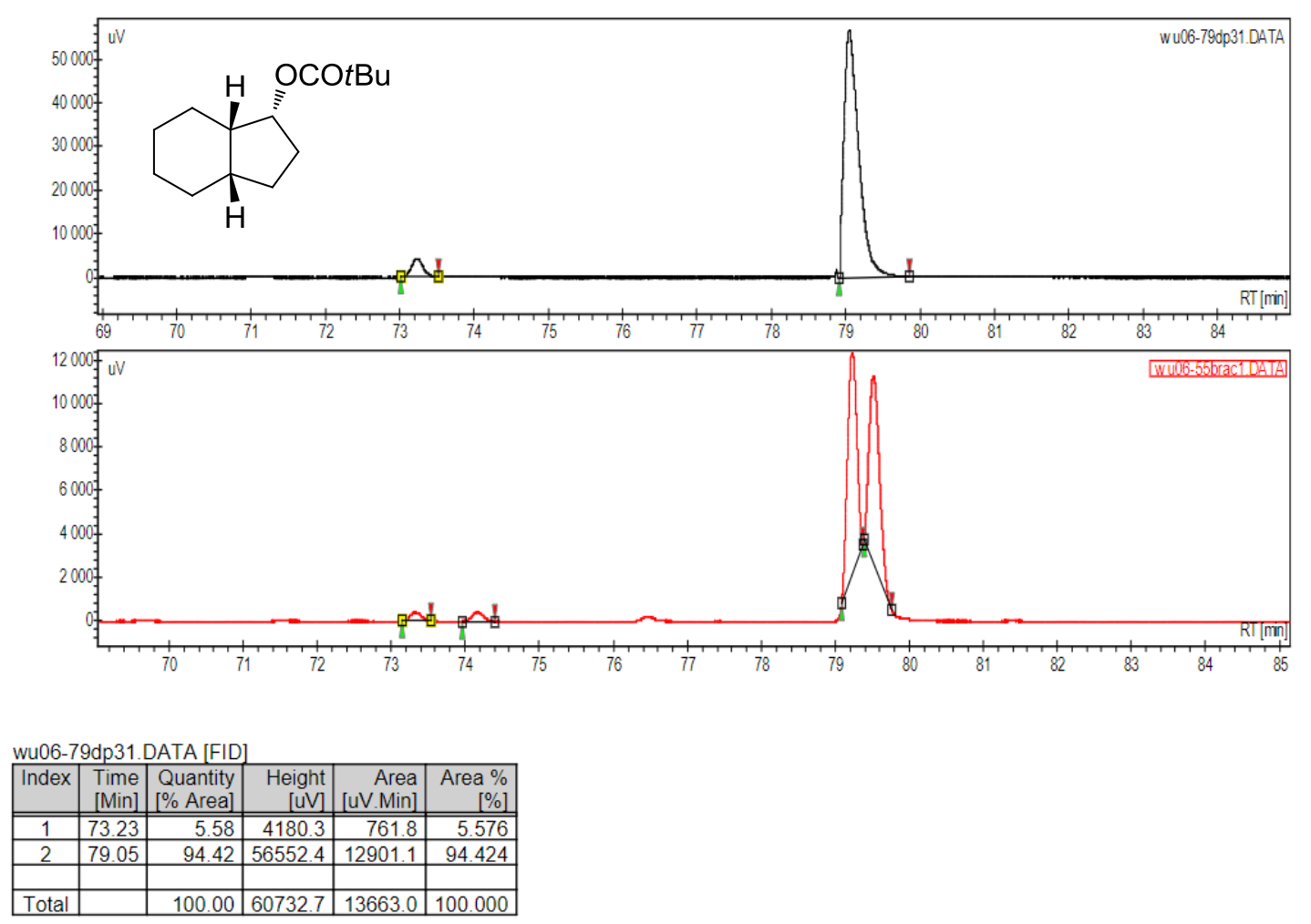

wu06-55brac1.DATA [FID]
\begin{tabular}{|c|r|r|r|r|r|}
\hline Index & $\begin{array}{r}\text { Time } \\
{[\text { Min] }]}\end{array}$ & $\begin{array}{r}\text { Quantity } \\
{[\% \text { Area] }}\end{array}$ & $\begin{array}{r}\text { Height } \\
{[\text { uV] }]}\end{array}$ & $\begin{array}{r}\text { Area } \\
{[\text { uV.Min] }}\end{array}$ & $\begin{array}{r}\text { Area \% } \\
{[\%]}\end{array}$ \\
\hline \hline 1 & 73.32 & 2.21 & 390.7 & 67.1 & 2.213 \\
\hline 2 & 74.17 & 2.43 & 411.5 & 73.5 & 2.425 \\
\hline 3 & 79.23 & 48.98 & 10342.8 & 1484.9 & 48.982 \\
\hline 4 & 79.52 & 46.38 & 8637.1 & 1405.9 & 46.379 \\
\hline & & & & & \\
\hline Total & & 100.00 & 19782.1 & 3031.4 & 100.000 \\
\hline
\end{tabular}
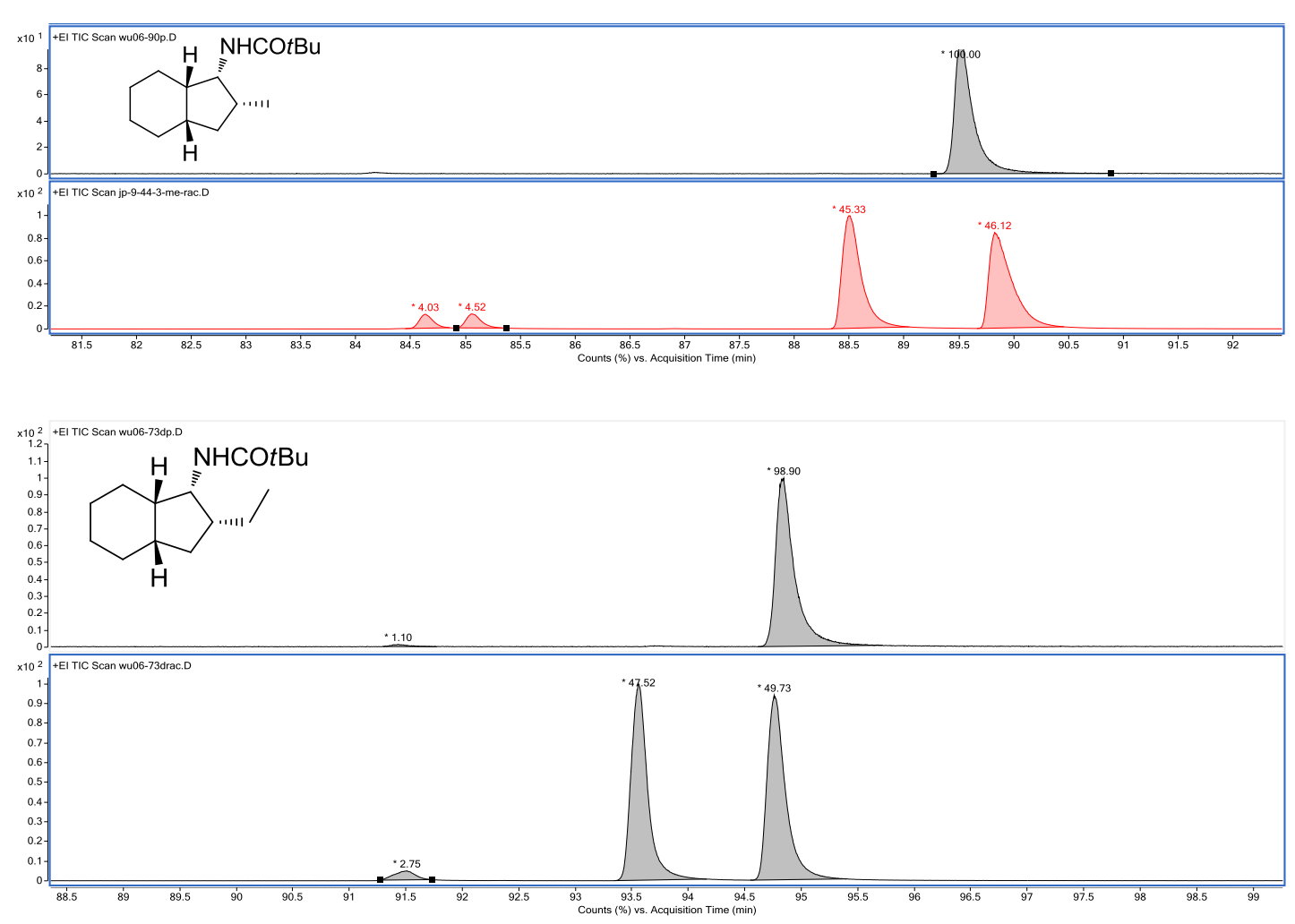

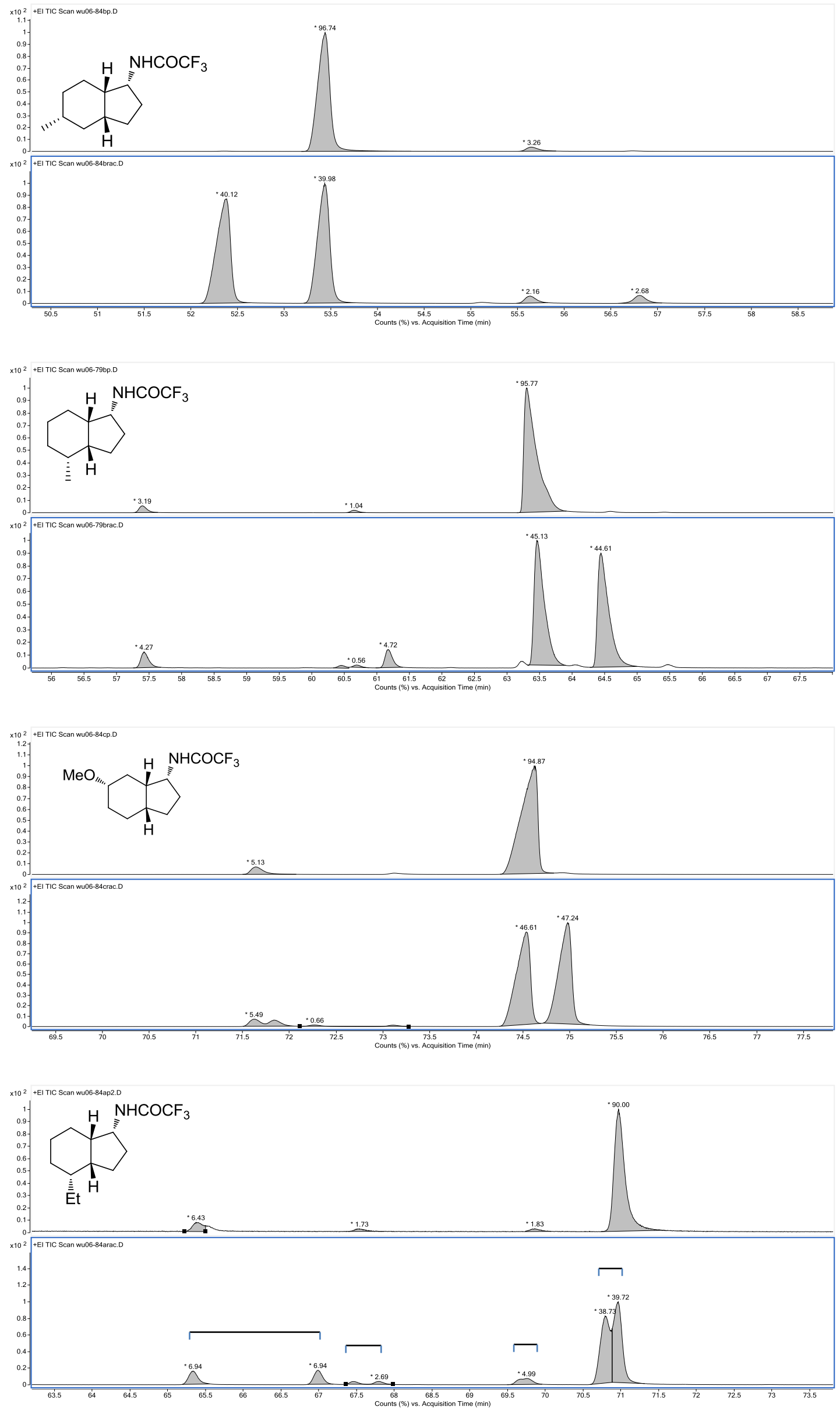

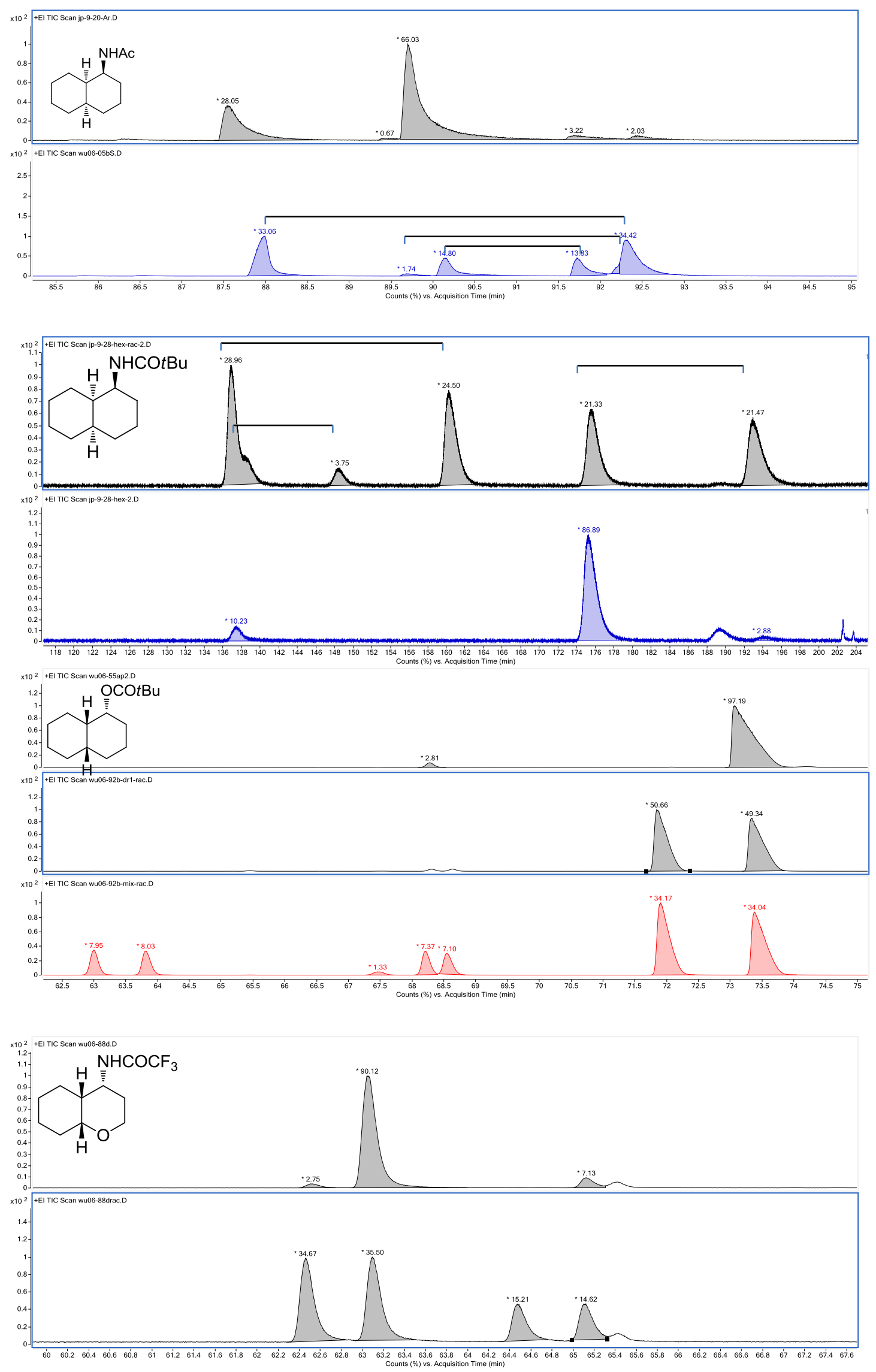

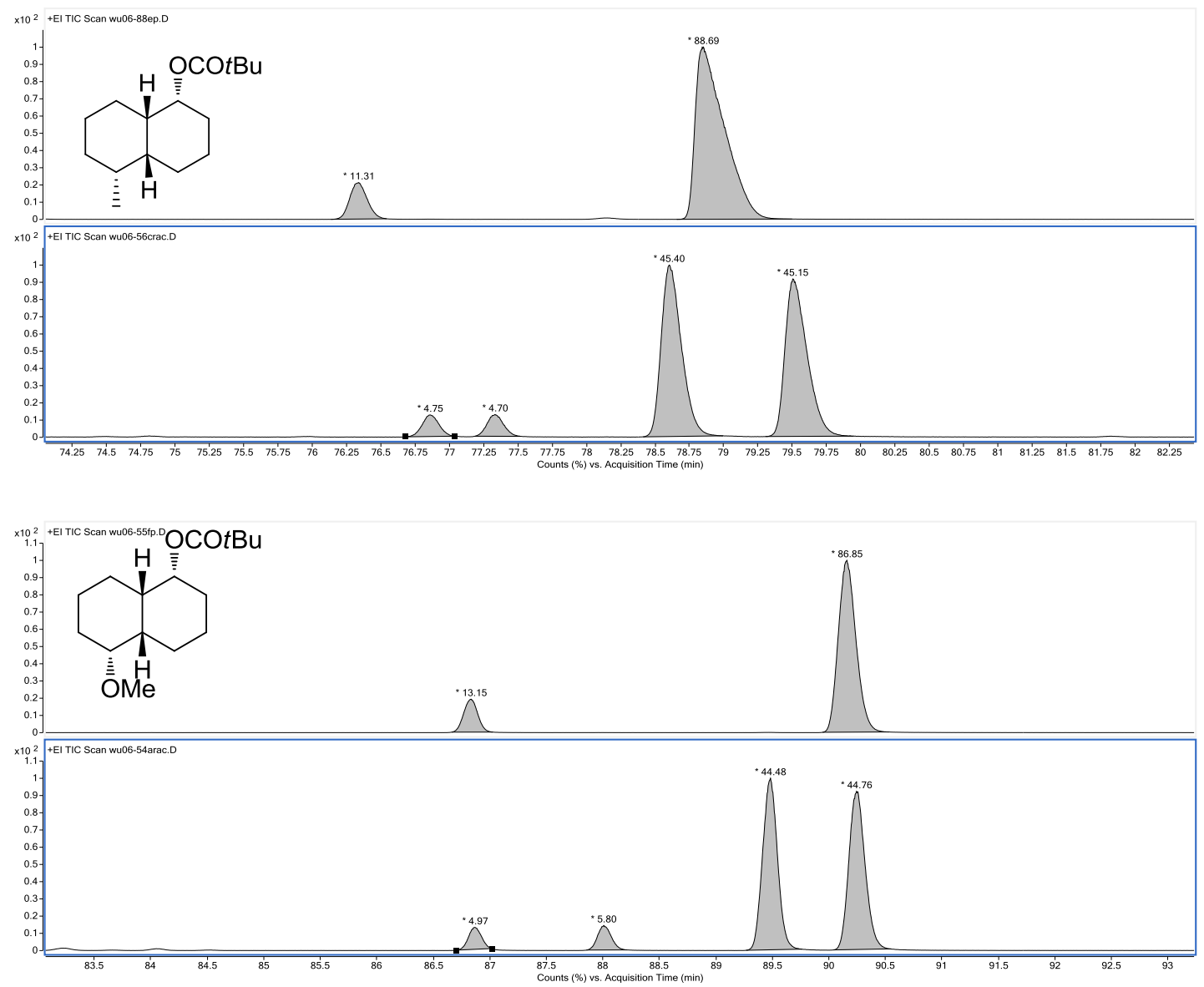

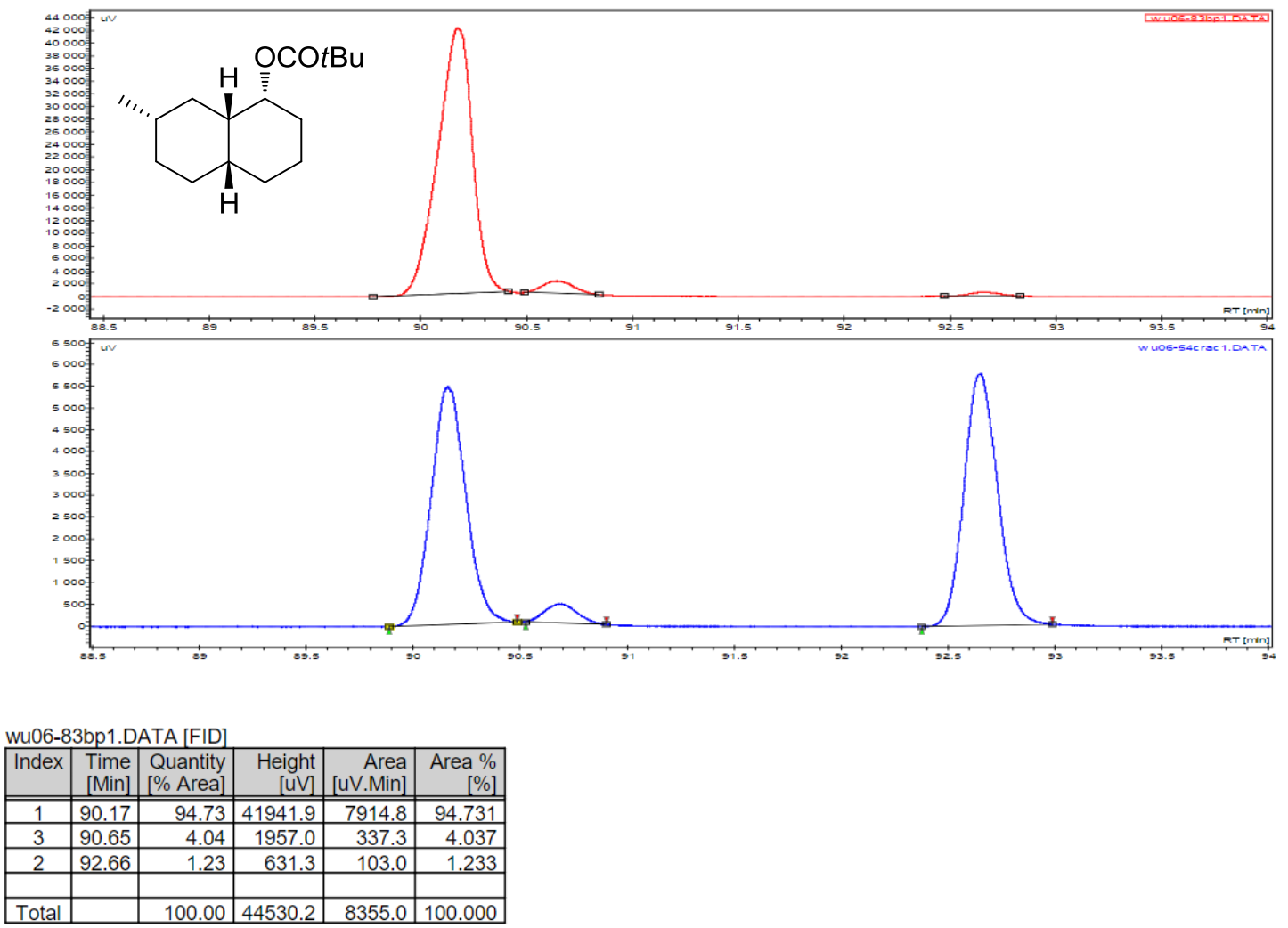

wu06-54crac1.DATA [FID]
\begin{tabular}{|c|r|r|r|r|r|}
\hline Index & $\begin{array}{r}\text { Time } \\
\text { [Min] }\end{array}$ & $\begin{array}{r}\text { Quantity } \\
\text { [\% Area] }\end{array}$ & $\begin{array}{r}\text { Height } \\
\text { [uV] }\end{array}$ & $\begin{array}{r}\text { Area } \\
\text { [uV.Min] }\end{array}$ & $\begin{array}{r}\text { Area \% } \\
{[\%]}\end{array}$ \\
\hline \hline 1 & 90.16 & 47.65 & 5465.6 & 1024.1 & 47.648 \\
\hline 2 & 90.70 & 3.59 & 437.7 & 77.1 & 3.588 \\
\hline 3 & 92.65 & 48.76 & 5763.2 & 1048.1 & 48.764 \\
\hline & & & & & \\
\hline Total & & 100.00 & 11666.5 & 2149.3 & 100.000 \\
\hline
\end{tabular}




\section{References}

1. (a) Jiang, Q., Xiao, D., Zhang, Z., Cao, P. \& Zhang, X. Highly Enantioselective Hydrogenation of Cyclic Enol Acetates Catalyzed by a Rh-PennPhos Complex. Angew. Chem. Int. Ed. 1999, 38, 516-518. (b) Zhang, W.; Zhang, X., Synthesis of Triphosphorous Bidentate Phosphine-Phosphoramidite Ligands: Application in the Highly Enantioselective Hydrogenation of ortho-Substituted Aryl Enamides. Angew. Chem. Int. Ed. 2006, 45, 5515-5518.

2. Ensign, S. C., Vanable, E. P., Kortman, G. D., Weir, L. J. \& Hull, K. L. AntiMarkovnikov Hydroamination of Homoallylic Amines. J. Am. Chem. Soc. 2015, 137, 13748-13751.

3. Oro, L. A., Valderrama, M., Cifuentes, P., Foces-Foces, C. \& Cano, F. H. Azulene as a ligand in cationic rhodium and iridium complexes. Crystal structure of [Rh(TFB)(az)]PF6. J. Organomet. Chem. 1984, 276, 67-77.

4. Burk, M. J., Casy, G. \& Johnson, N. B. A Three-Step Procedure for Asymmetric Catalytic Reductive Amidation of Ketones. J. Org. Chem. 1998, 63, 6084-6085.

5. Massaro, L. et al. Stereodivergent Synthesis of Trisubstituted Enamides: Direct Access to Both Pure Geometrical Isomers. J. Org. Chem. 2019, 84, 13540-13548.

6. Guan, Z.-H., Zhang, Z.-Y., Ren, Z.-H., Wang, Y.-Y. \& Zhang, X. Synthesis of Enamides via CuI-Catalyzed Reductive Acylation of Ketoximes with NaHSO3. J. Org. Chem. 2011, 76, 339-341.

7. Yousuf, M. \& Adhikari, S. One-Pot Synthesis of 3-Substituted 2-Arylpyrrole in Aqueous Media via Addition-Annulation of Arylboronic Acid and Substituted Aliphatic Nitriles. Org. Lett. 2017, 19, 2214-2217.

8. Guan, Z.-H., Huang, K., Yu, S. \& Zhang, X. Synthesis of Enamides via Rh/CCatalyzed Direct Hydroacylation of Ketoximes. Org. Lett. 2009, 11, 481-483.

9. Li, B.-J. et al. Cross-Coupling of Alkenyl/Aryl Carboxylates with Grignard Reagent via Fe-Catalyzed C-O Bond Activation. J. Am. Chem. Soc. 2009, 131, 14656-14657.

10. Yang, H., Wang, E., Yang, P., Lv, H. \& Zhang, X. Pyridine-Directed Asymmetric Hydrogenation of 1,1-Diarylalkenes. Org. Lett. 2017, 19, 50625065.

11. Zhu, G., Chen, Z. \& Zhang, X. Highly Efficient Asymmetric Synthesis of $\beta$ Amino Acid Derivatives via Rhodium-Catalyzed Hydrogenation of $\beta$ (Acylamino)acrylates. J. Org. Chem. 1999, 64, 6907-6910.

12. Storch, G. \& Trapp, O. Temperature-Controlled Bidirectional Enantioselectivity in a Dynamic Catalyst for Asymmetric Hydrogenation. Angew. Chem. Int. Ed. 2015, 54, 3580-3586.

13. Zhang, Z., Zhu, G., Jiang, Q., Xiao, D. \& Zhang, X. Highly Enantioselective Hydrogenation of Cyclic Enamides Catalyzed by a Rh-PennPhos Catalyst. J. Org. Chem. 1999, 64, 1774-1775.

14. Ranade, V. S., Consiglio, G. \& Prins, R. Functional-Group-Directed Diastereoselective Hydrogenation of Aromatic Compounds. 21. J. Org. Chem. 2000, 65, 1132-1138.

15. Solladié-Cavallo, A., Ahmed, B., Schmitt, M. \& Garin, F. Heterogeneous hydrogenation of 1-naphtol and 2-naphtol over $\mathrm{Ru} / \mathrm{Al}_{2} \mathrm{O}_{3}$ : a simple $1 \mathrm{H}$ NMR 
method for determination of the diastereoselectivity. C. $R$. Chimie. 2005, 8 , 1975-1980.

16. Reeves, J. T.; Tan, Z.; Han, Z. S.; Li, G.; Zhang, Y.; Xu, Y.; Reeves, D. C.; Gonnella, N. C.; Ma, S.; Lee, H.; Lu, B. Z.; Senanayake, C. H., Direct TitaniumMediated Conversion of Ketones into Enamides with Ammonia and Acetic Anhydride. Angew. Chem. Int. Ed. 2012, 51, 1400-1404. 